\title{
Charge Density Distribution in Low-Valent Tetreles
}

\author{
Dissertation zur Erlangung des \\ mathematisch-naturwissenschaftlichen Doktorgrades \\ "Doctor rerum naturalium" \\ der Georg-August-Universität Göttingen \\ im Promotionsprogramm Chemie der \\ Georg-August University School of Science (GAUSS)
}

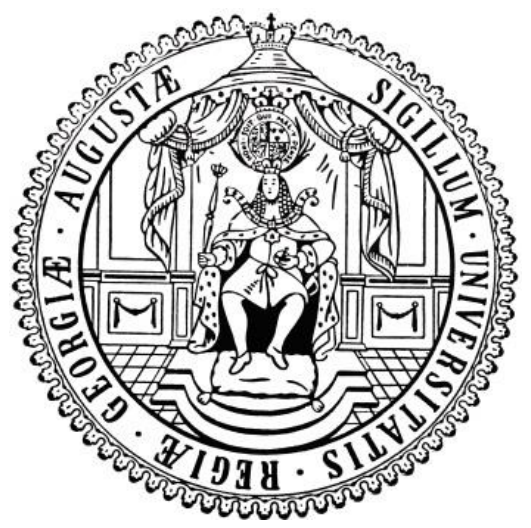

vorgelegt von

Daniel Kratzert

aus Friedrichshafen

Göttingen, 2013 


\section{Betreuungsausschuss}

Prof. Dr. Dietmar Stalke, Institut für Anorganische Chemie

Prof. Dr. George M. Sheldrick, Institut für Anorganische Chemie

\section{Mitglieder der Prüfungskommission}

Referent: Prof. Dr. Dietmar Stalke

Korreferent: Prof. Dr. George M. Sheldrick

weitere Mitglieder

Prof. Dr. Dr. h.c. mult. Herbert W. Roesky, Institut für Anorganische Chemie

Dr. Inke Siewert, Institut für Anorganische Chemie

Dr. Heidrun Sowa, GZG, Abt. Kristallographie

Jun.-Prof. Dr. Thomas Waitz, Institut für Anorganische Chemie

Tag der mündlichen Prüfung: 09.04.2013 


\section{Danksagung}

Die vorliegende Arbeit wurde in der Zeit von Juni 2008 bis April 2013 im Arbeitskreis Stalke am Institut für Anorganische Chemie der Universität Göttingen angefertigt. An dieser Stelle möchte ich allen danken, die zum Gelingen dieser Arbeit beigetragen haben.

An erster Stelle gilt mein Danke meinem Doktorvater Dietmar Stalke für sein grenzenloses Vertrauen in meine Arbeit, die Bereitstellung von einmaligen technischen Möglichkeiten für die Erforschung kristallographischer Probleme und die Möglichkeit viele interessante Fachkonferenzen weltweit zu besuchen.

Allen Angestellten der Feinmechanik- und Elektrowerkstatt des Institutes für Anorganische Chemie danke ich für die tolle Zusammenarbeit. Vor allem die Arbeit von Ha. Jo. Heymel, Benjamin Bock, Mariusz Burzynski, Bernd Wichmann, Rainer Ehrhardt und Stephan Dullnig war mir eine große Hilfe.

Allen gegenwärtigen und ehemaligen Arbeitskreismitgliedern danke ich für die Unterstützung und den Spaß während, als auch außerhalb der Arbeit.

Ausserdem danke ich auch:

Dirk Leußer danke ich für die Einführung in die Kristallographische Theorie und Praxis sowie topologische Untersuchung von Elektronendichte. Ohne diese gute Einführung wäre diese Arbeit so sicher nicht entstanden.

Birger Dittrich und Julian Holstein danke ich für die große Hilfe bei jeglichen Fragen zu Invariomen.

Regine Herbst-Irmer und George Sheldrick für viele gute Diskussionen in allen fachlichen Bereichen.

Meinen Korrektoren Nina Lock, Eva Müller-Stüler, Lennard Krause, Hilke Wolf und Jakob Hey. Ihr habt einen super Job gemacht.

Meinem Laborkollegen Markus Granitzka für die gute Zusammenarbeit im Labor und überall sonst.

Jakob Hey für die gute Zusammenarbeit an den Maschinen und für schöne Touren mit dem Rad und alles Mögliche weitere...

Reent Michel für leckere norddeutsche Abendessen und Grünkohl mit Pinkel.

Felix Engelhardt und Stefan Deuerlein danke ich für die gute Zusammenarbeit bei der Administration des Abteilungsnetzes.

Heike Tappe danke ich für die kompetente Unterstützung in allen Verwaltungsdingen.

Meinen Kooperationspartnern danke ich für viele interessante kristallographische Probleme zum knobeln.

Daniel Stern und Holger Ott danke ich für die gute Zusammenarbeit in Göttingen und für die wertvolle technische Unterstützung bei allen Maschinenproblemen.

Martin Schlote für die perfekte Organisation des Chemikalienlagers und die Beschaffung aller Laborgeräte.

Meinen Bachelorstudenten und Praktikanten Christian Volkmann, Levke Deutsch, Florian Zurheide und Malte Schneemann für die gute Laborarbeit.

Dem Abteilungsgrill Modell „Oldenburg“ danke ich für die Leckere Bratwurst.

Vor allem danke ich meinen Eltern. Ohne eure Unterstützung und das grenzenlose Vertrauen wäre diese Arbeit sicher nicht entstanden. 
"We choose to go to the moon in this decade and do the other things, not because they are easy, but because they are hard, because that goal will serve to organize and measure the best of our energies and skills, because that challenge is one that we are willing to accept, one we are unwilling to postpone, and one which we intend to win, and the others, too."

John F. Kennedy 


\section{Content}

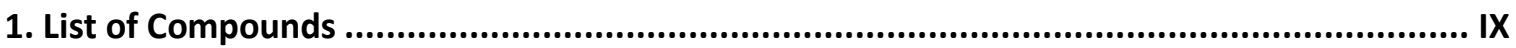

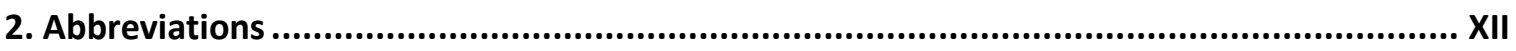

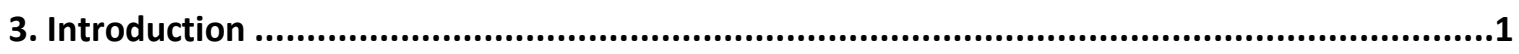

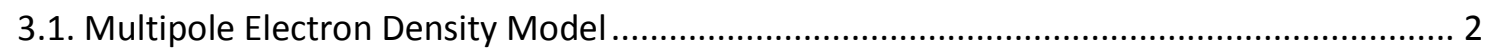

3.2. Electron Density Transfer from Databases ..................................................................... 3

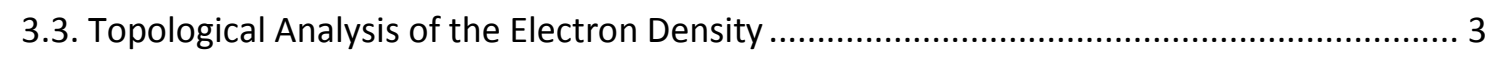

3.3.1. Quantum Theory of Atoms in Molecules ................................................................. 4

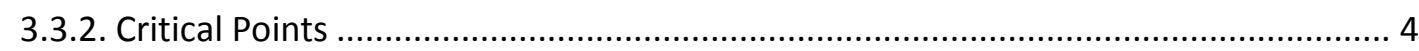

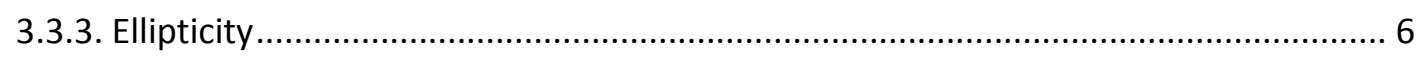

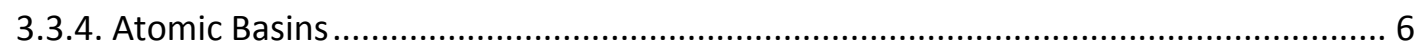

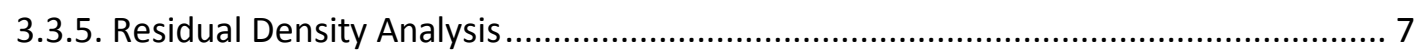

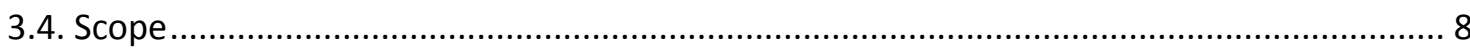

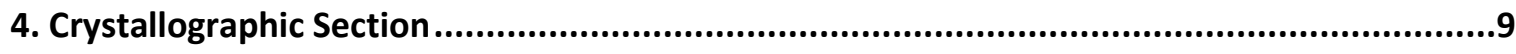

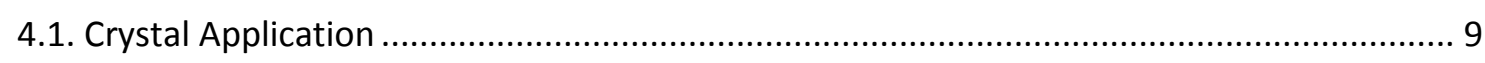

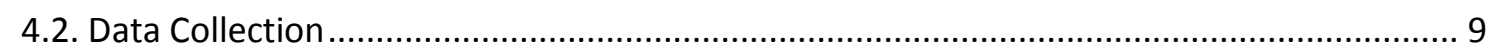

4.2.1. Data Collection for Routine Structures ............................................................... 9

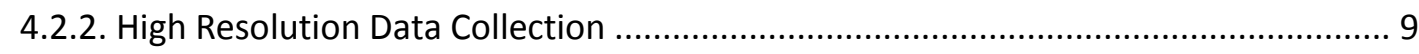

4.2.3. Judgment Criteria for Data Quality .................................................................... 11

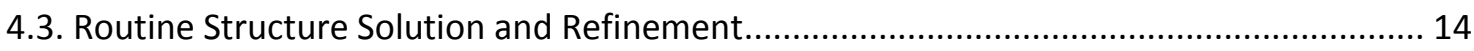

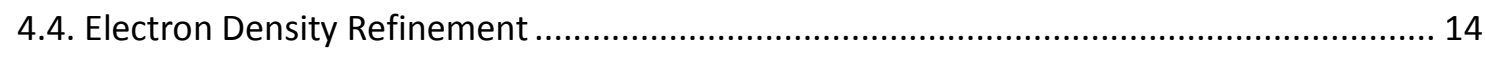

4.4.1. General Procedure for an Electron Density Starting Model .................................... 14

4.4.2. General Procedure for an Invariom Transfer ............................................................. 15

4.4.3. XD2006 Refinement Procedure ............................................................................... 16

4.4.4. Plotxd - a Tool for Structured Parameter Output from XD2006 ............................... 17

4.4.5. Refcheck - a Refinement Helper Tool.................................................................. 18

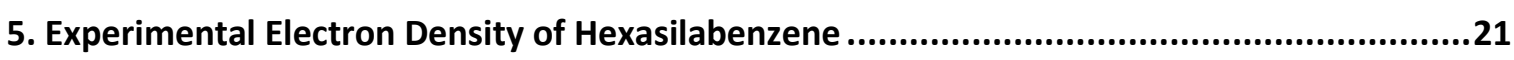

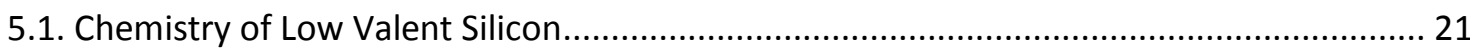

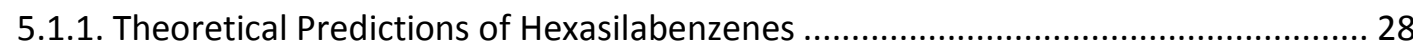

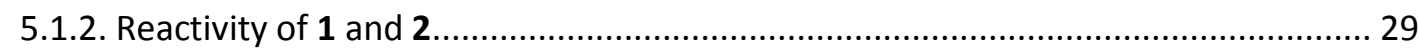

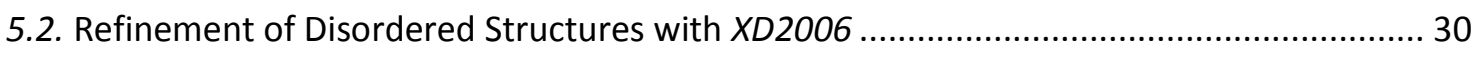

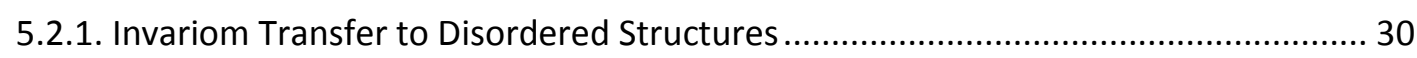

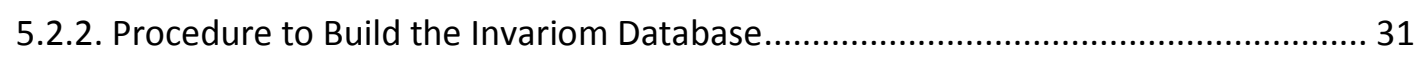

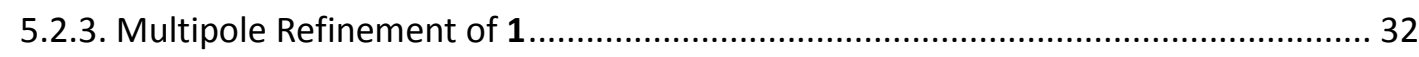

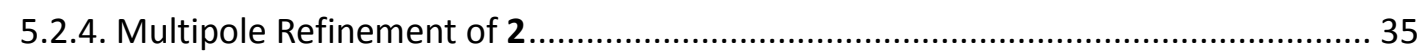

5.3. Topological Analysis of the Hexasilabenzene Isomer 1 ................................................... 37

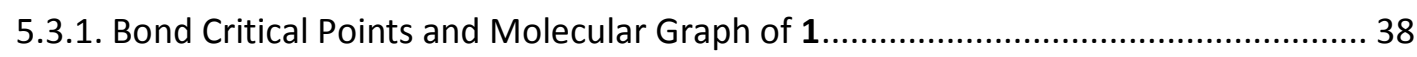

5.3.2. Integrated Charges and Valence Shell Charge Concentrations ................................ 39

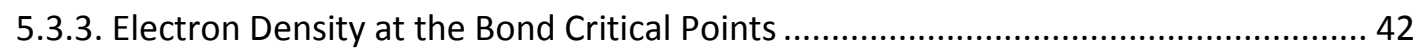

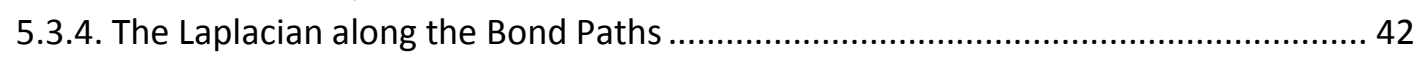

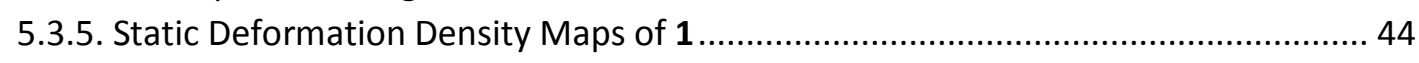




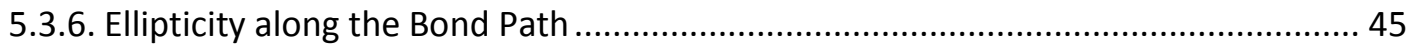

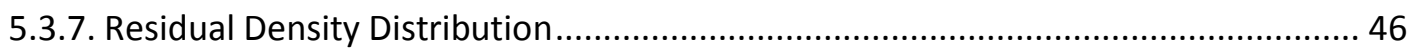

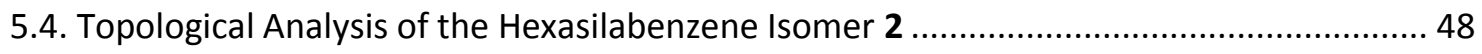

5.4.1. Bond Critical Points and Molecular Graph of 2 ...................................................... 48

5.4.2. Integrated Charges and Valence Shell Charge Concentrations .............................. 52

5.4.3. Electron Density at the Bond Critical Point............................................................... 54

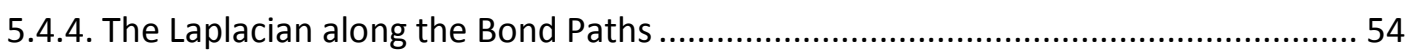

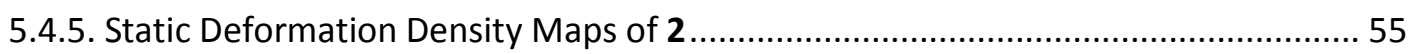

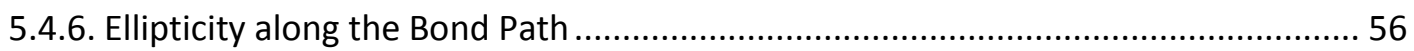

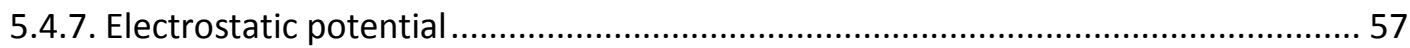

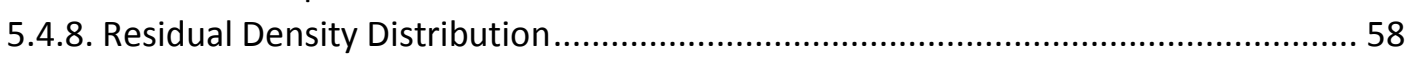

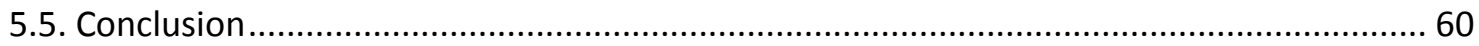

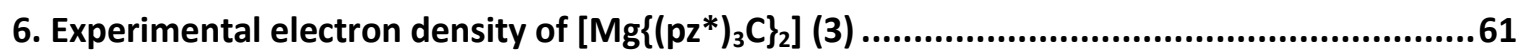

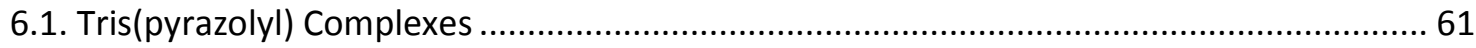

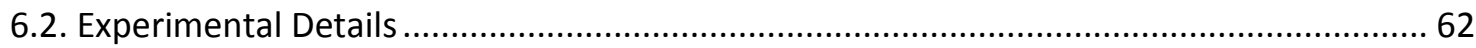

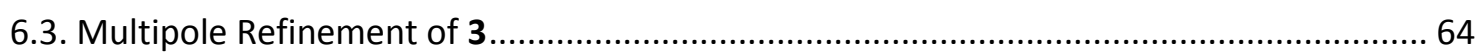

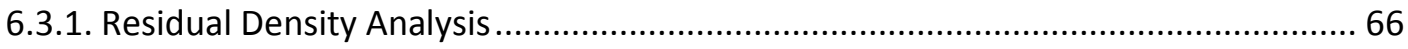

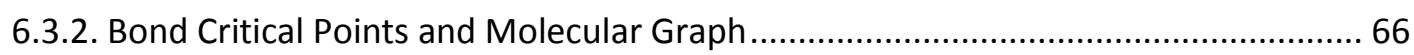

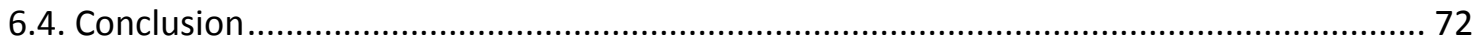

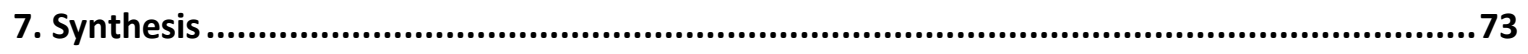

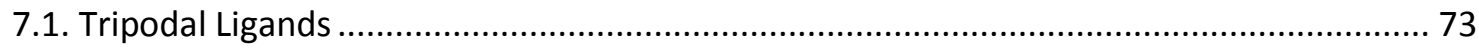

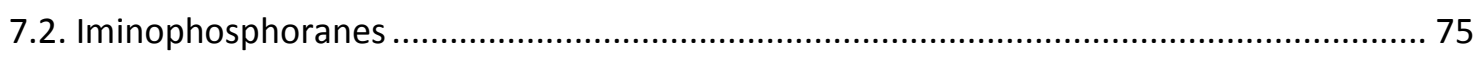

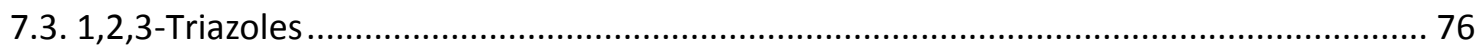

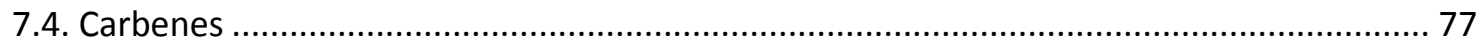

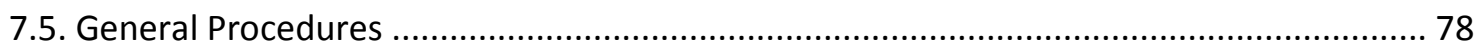

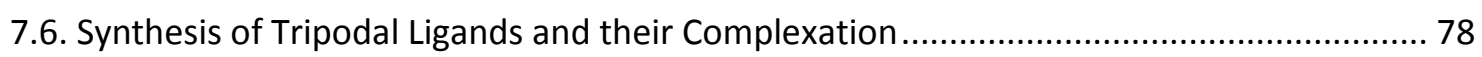

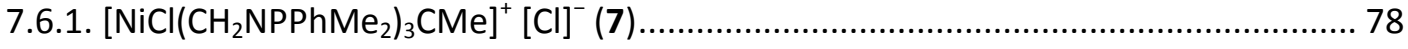

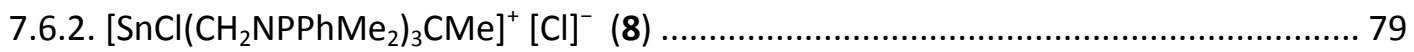

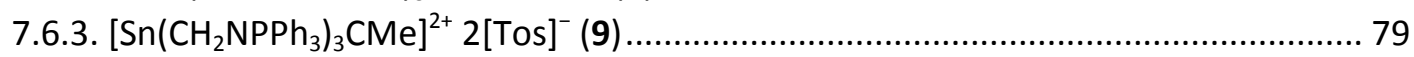

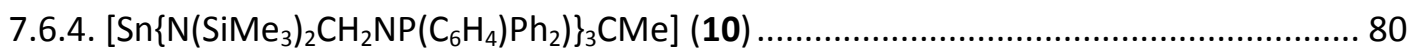

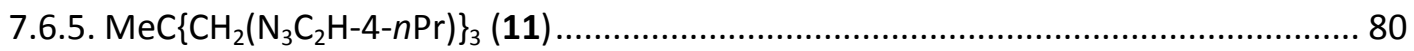

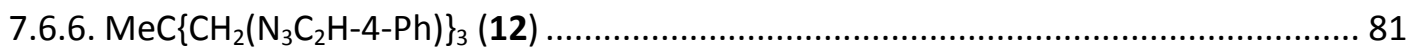

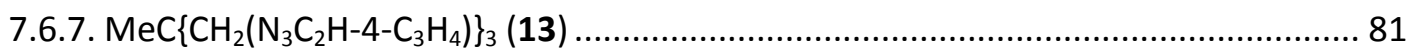

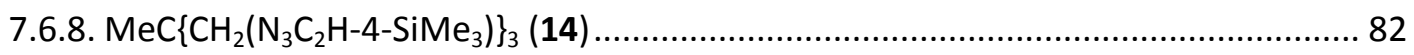

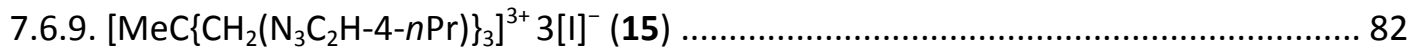

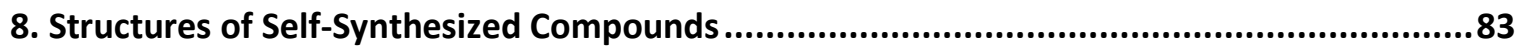

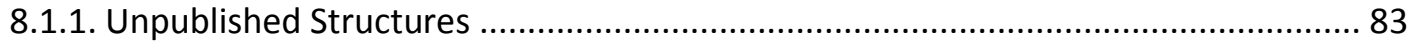

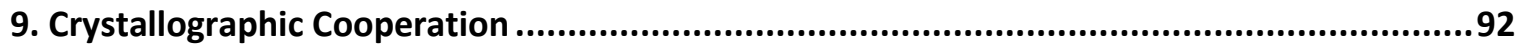

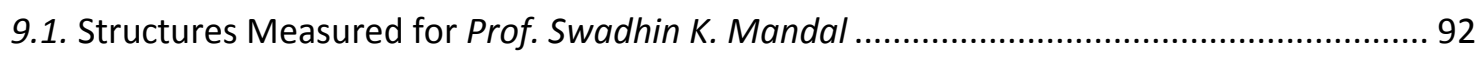

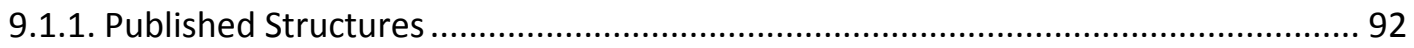




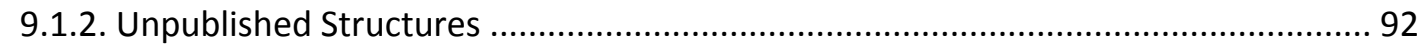

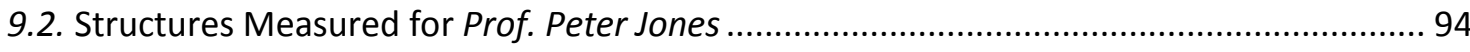

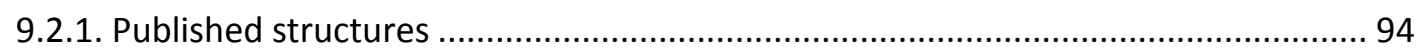

9.3. Structures Measured for Sakya S. Sen (Prof. H. W. Roesky) ................................................ 94

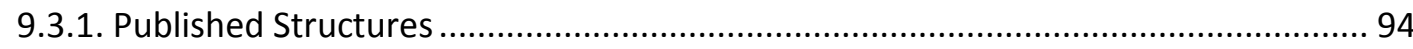

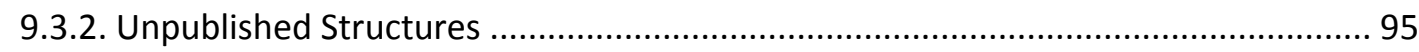

9.4. Structures Measured for Shabana Khan (Prof. H. W. Roesky) ............................................. 96

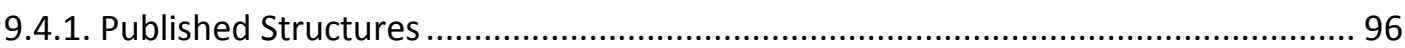

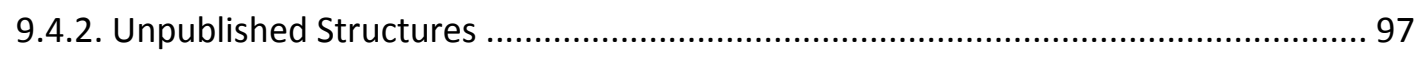

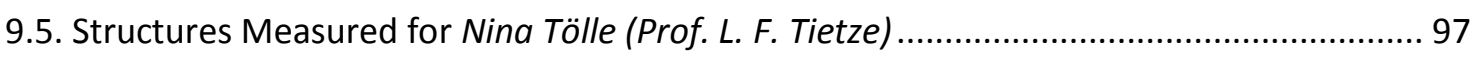

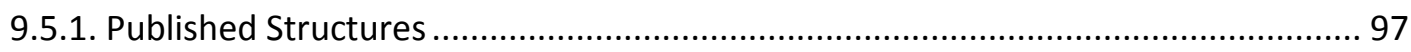

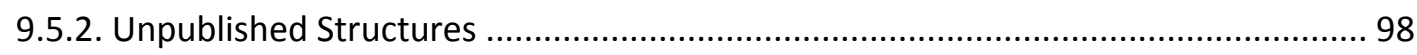

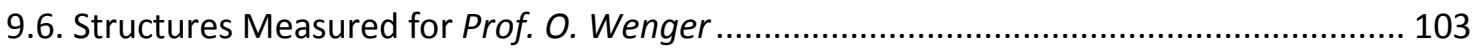

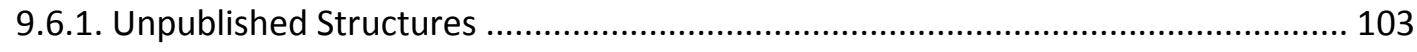

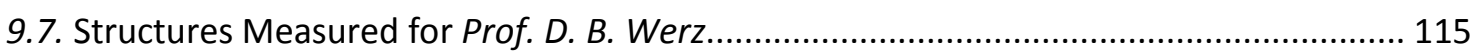

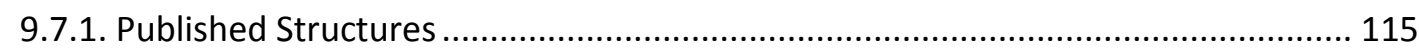

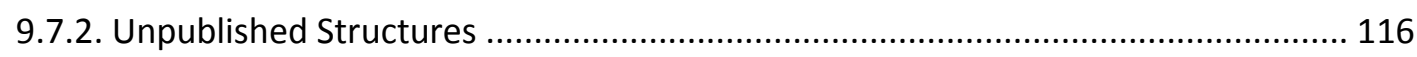

9.8. Structures Measured for Patrick Zark (Prof. T. Müller) ..................................................... 126

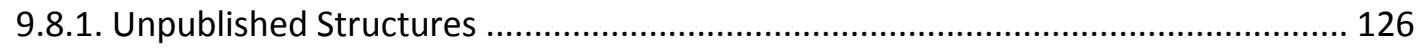

9.9. Structures Measured for Annika Stute (Prof. G. Erker) .................................................. 127

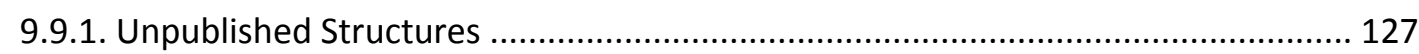

10. Appendix

10.1. Cross Hair Alignment for Bruker 3-circle Goniometers................................................ 131

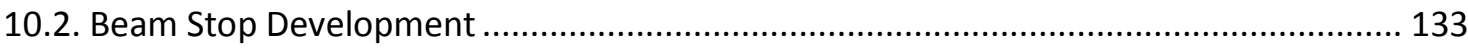

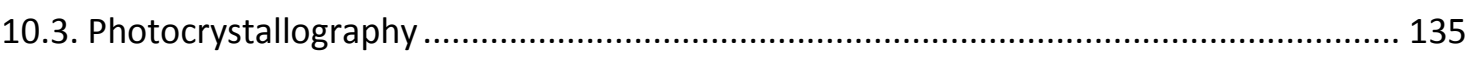

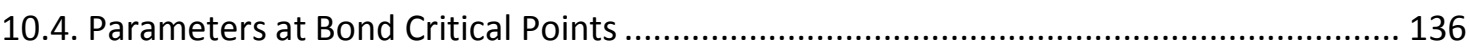

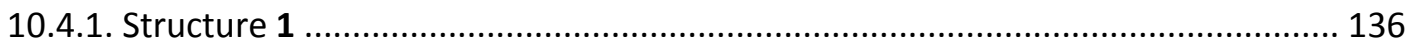

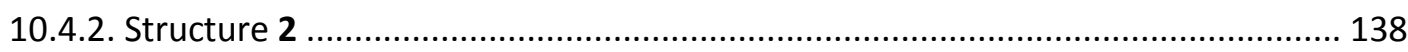

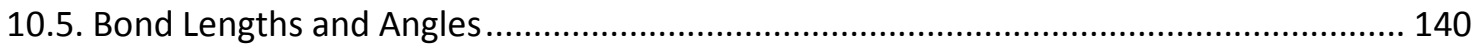

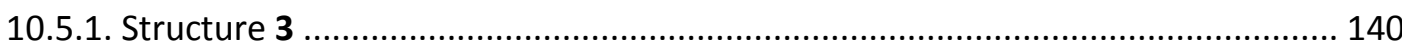

10.5.2. Atom Names of the Calculated Structure of 1 ................................................ 141

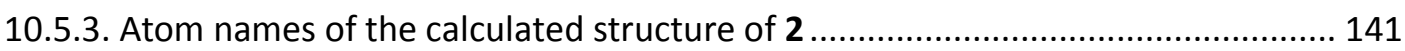

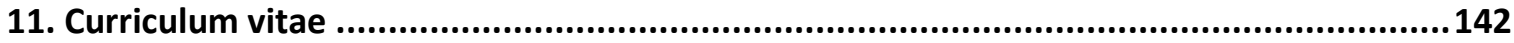

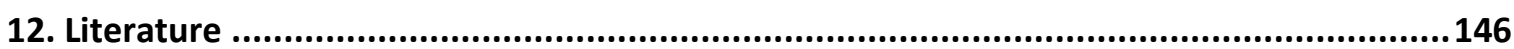

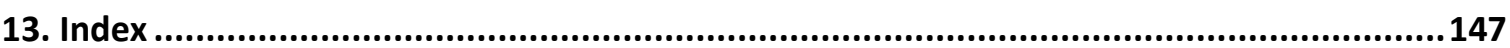




\section{List of Compounds}

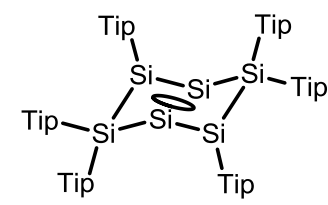

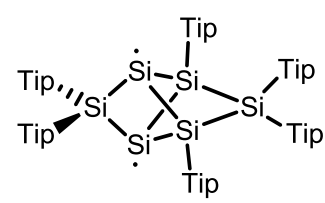

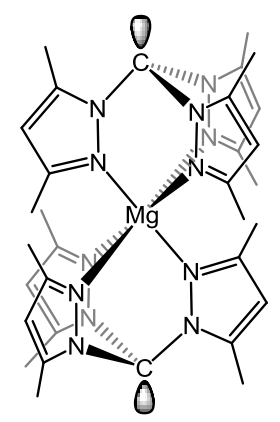<smiles>CC(CN)(CN)CN</smiles><smiles>CC(CN=P(C)(C)c1ccccc1)(CN=P(C)(C)c1ccccc1)CN=P(C)(C)c1ccccc1</smiles>

$1\left(\mathrm{TipSi}_{6}\right.$

$2(\mathrm{TipSi})_{6}$

$3\left[\mathrm{Mg}\left\{(\mathrm{pz} *)_{3} \mathrm{C}\right\}_{2}\right]$

$5 \mathrm{MeC}\left(\mathrm{CH}_{2} \mathrm{NPPhMe}_{2}\right)_{3}$ 
<smiles>CC(CN=[PH](c1ccccc1)c1ccccc1)(CN=P(c1ccccc1)(c1ccccc1)c1ccccc1)CN=P(c1ccccc1)(c1ccccc1)c1ccccc1</smiles>

$6 \mathrm{MeC}\left(\mathrm{CH}_{2} \mathrm{NPPh}_{3}\right)_{3}$

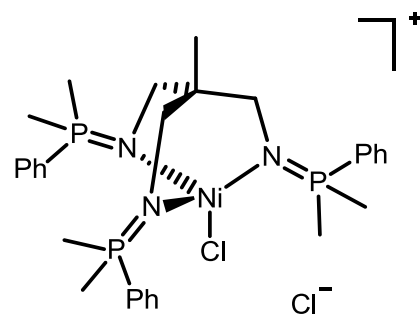

$7\left[\mathrm{NiCl}\left(\mathrm{CH}_{2} \mathrm{NPPhMe}_{2}\right)_{3} \mathrm{CMe}\right]^{+}[\mathrm{Cl}]^{-}$

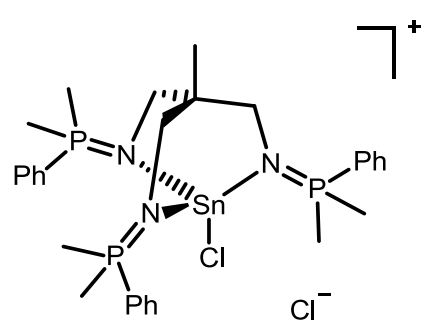

$8\left[\mathrm{SnCl}\left(\mathrm{CH}_{2} \mathrm{NPPhMe}_{2}\right)_{3} \mathrm{CMe}\right]^{+}[\mathrm{Cl}]^{-}$

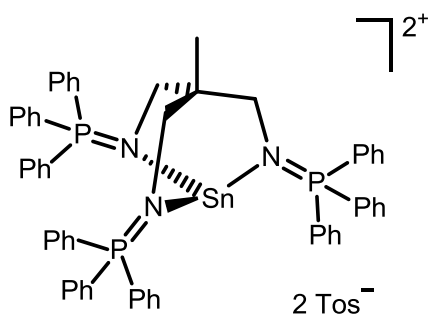

$9\left[\mathrm{Sn}\left(\mathrm{CH}_{2} \mathrm{NPPh}_{3}\right)_{3} \mathrm{CMe}\right]^{2+} 2[\mathrm{Tos}]^{-}$<smiles>CCN(C)[SnH]1N=P(c2ccccc2)(c2ccccc2)c2ccccc21</smiles>

$\left.10\left[\mathrm{Sn}\left\{\mathrm{N}\left(\mathrm{SiMe}_{3}\right)_{2} \mathrm{CH}_{2} \mathrm{NP}\left(\mathrm{C}_{6} \mathrm{H}_{4}\right) \mathrm{Ph}_{2}\right)\right\}_{3} \mathrm{CMe}\right]$ 
<smiles>CCCc1cn(CC(C)(Cn2cc(CCC)nn2)Cn2cc(CCC)nn2)nn1</smiles>

$11 \mathrm{MeC}\left\{\mathrm{CH}_{2}\left(\mathrm{~N}_{3} \mathrm{C}_{2} \mathrm{H}-4-n \mathrm{Pr}\right)\right\}_{3}$<smiles>CC(Cn1cc(-c2ccccc2)nn1)(Cn1cc(-c2ccccc2)nn1)Cn1cc(-c2ccccc2)nn1</smiles>

$12 \mathrm{MeC}\left\{\mathrm{CH}_{2}\left(\mathrm{~N}_{3} \mathrm{C}_{2} \mathrm{H}-4-\mathrm{Ph}\right)\right\}_{3}$<smiles>CC(Cn1cc(C2CC2)nn1)(Cn1cc(C2CC2)nn1)Cn1cc(C2CC2)nn1</smiles>

$13 \mathrm{MeC}\left\{\mathrm{CH}_{2}\left(\mathrm{~N}_{3} \mathrm{C}_{2} \mathrm{H}-4-\mathrm{C}_{3} \mathrm{H}_{4}\right)\right\}_{3}$<smiles>CC(Cn1cc(S(C)(=O)=O)nn1)(Cn1cc(S(C)(=O)=O)nn1)Cn1cc(S(C)(=O)=O)nn1</smiles>

$14 \mathrm{MeC}\left\{\mathrm{CH}_{2}\left(\mathrm{~N}_{3} \mathrm{C}_{2} \mathrm{H}-4-\mathrm{SiMe}_{3}\right)\right\}_{3}$<smiles></smiles>

$15\left[\mathrm{MeC}\left\{\mathrm{CH}_{2}\left(\mathrm{~N}_{3} \mathrm{C}_{2} \mathrm{H}-4-n \mathrm{Pr}\right)_{3}\right\}\right]^{3+} 3[\mathrm{I}]^{-}$ 


\section{Abbreviations}

$\begin{array}{ll}\nabla^{2} \rho(\boldsymbol{r})=L(\boldsymbol{r}) & \text { Laplacian field } \\ \text { ADP } & \text { Atomic Displacement Parameter } \\ \text { AIM } & \text { Atoms In Molecules } \\ \text { CCD } & \text { Charge Coupled Device } \\ \text { CMOS } & \text { Complementary Metal Oxide Semiconductor } \\ \text { DMSDA } & \text { Differences of Mean-Squares Displacement Amplitudes } \\ \text { Gof } & \text { Goodness of Fit } \\ \text { HOMO } & \text { Highest Occupied Molecular Orbital } \\ \text { iso } & \text { isotropic } \\ \text { IAM } & \text { Independent Atom Model } \\ \text { LUMO } & \text { Lowest Unoccupied Molecular Orbital } \\ \text { Me } & \text { methyl } \\ \text { MO } & \text { molecular orbital } \\ \text { MSDA } & \text { Mean Square Displacement Amplitude } \\ n B u L i & n \text {-Butyllithium } \\ \text { Ph } & \text { phenyl } \\ \text { Pr } & \text { Propyle group } \\ t \text { Bu } & \text { tert-Butyl group } \\ t \text { BuLi } & \text { tert-Butyllithium } \\ \text { THF } & \text { Triisopropyl phenyl group } \\ \text { Tip } & \text { Valence Bond } \\ \text { TMS } & \text { VB }\end{array}$





\section{Introduction}

X-ray diffraction was first used only to determine the positions of atoms in the crystal lattice. Since the first diffraction patterns of Max von Laue in $1912^{[1-3]}$ the method made an enormous progress. The development of single crystal diffractometers in the 1960s and the invention of modern computers made it possible to gain more detailed insights in the description of chemical bonding. ${ }^{[4-6]}$ Accurate measurement of the charge density in a crystal became feasible in that time. The Independent Atom Model (IAM) was used to describe the charge density of spherically averaged ground-state atoms in a crystal. ${ }^{[7]}$ In this model every type of atom is described by a specific radial exponential distribution of the electron density. The Fourier transformation of the electron density for atom $i$ is the atomic form factor $f_{i}(\boldsymbol{h})$ with $f(0)=Z_{i}$, where $\boldsymbol{h}$ is the reciprocal scattering vector and $Z$ is the atomic number of atom $i$ with $\boldsymbol{h}=h \boldsymbol{a}^{*}+k \boldsymbol{b}^{*}+l \boldsymbol{c}^{*}$. Hence, the atomic form factor is taking into account the different scattering power of the elements.

$$
f(\theta, \lambda)=f^{0}(\theta)+\Delta f^{\prime}(\lambda)+i \Delta f^{\prime \prime}(\lambda)
$$

The atomic form factor $f_{i}(\boldsymbol{h})$ is consisting of a real and an imaginary part. The $f^{\prime}$ and the $f^{\prime \prime}$ part of the scattered $X$-ray radiation with the wave length $\lambda$ is often called the anomalous signal. The proportion of the anomalous signal gets higher for greater scattering angles $\theta$. The imaginary part $f^{\prime \prime}$ is important for the clear determination of the absolute structure and absolute-configuration of crystal structures. ${ }^{[8]}$ Summation over $N$ atoms in the unit cell gives the structure factor $F(\boldsymbol{h}) .{ }^{[9-10]}$

$$
F(\boldsymbol{h})=\sum_{i=1}^{N} f_{i}(\boldsymbol{h}) \cdot e^{2 \pi i \boldsymbol{h} r_{i}}
$$

The structure factor $F(\boldsymbol{h})$ describes the way in which an incident beam of $\mathrm{X}$-ray light is scattered by the atoms of a crystal unit cell. The Fourier transformation of $F(\boldsymbol{h})$ gives the electron density $\rho(\boldsymbol{r})$. The structure factor by definition is the Fourier transformation of the charge density $\rho(\boldsymbol{r})$, and the summation of the individual atomic contributions is approximately $\rho(\boldsymbol{r})$ of the molecule.

$$
\rho(\boldsymbol{r})=\frac{1}{V} \sum_{h, k, l} F(\boldsymbol{h}) e^{-2 \pi i \boldsymbol{h} \boldsymbol{r}}
$$

To build up a molecule from the density an important assumption must be made. In the independent atom model (IAM) atoms are assigned to the biggest maxima in the electron density. The reconstruction of the crystal structure from its diffraction image usually relies on the assumption that the atomic contribution dominates the total scattering. Bonding density between the atoms is ignored. Since the electrons are mostly localized around the nuclei, their local distributions are fairly well described by individual atomic densities. The IAM electron density is described as a convolution of a static charge density and a dynamical term. It is expressed as follows, where $\rho^{0}$ is the static electron density of the $k^{\text {th }}$ scatterer at the equilibrium position $\boldsymbol{R}^{0}, \boldsymbol{R}$ is the actual position, and $P$ is the probability density function describing the vibrational displacement $\boldsymbol{u}$ of the $k^{\text {th }}$ center.

$$
\begin{gathered}
\rho_{I A M}(\boldsymbol{r})=\int_{k}^{N} \rho^{0}\left(\boldsymbol{r}-\boldsymbol{R}^{0}-\boldsymbol{u}\right) P(\boldsymbol{u}) d \boldsymbol{u}=\rho(\boldsymbol{u}) \otimes P(\boldsymbol{u}) \\
\boldsymbol{u}=\boldsymbol{R}-\boldsymbol{R}^{0}
\end{gathered}
$$


In a real crystal there is always motion of the atoms, consisting of lattice vibrations and thermal movement of the atoms in a molecule. The correct deconvolution of motion and density is very important for the success of the X-ray experiment. This thermal effect is introduced into the structure factor equation by a factor that serves to attenuate the atomic scattering factor,

$$
f_{i}=f_{0} e^{-B \frac{\left(\sin ^{2} \theta\right)}{\lambda^{2}}}
$$

where the temperature factor (Debye-Waller-factor) B, is the related mean-square amplitude of the vibration and $\left\langle u^{2}\right\rangle$ the isotropic mean-square displacement. ${ }^{[11]}$

$$
B=8 \pi^{2} U=8 \pi^{2}\left\langle u^{2}\right\rangle
$$

In the harmonic approximation

$$
P(\boldsymbol{u})=(2 \pi)^{-3 / 2}|U|^{-1 / 2} e^{\left(-\frac{1}{2} \boldsymbol{u}^{t} U^{-1} \boldsymbol{u}\right)}
$$

is a Gaussian thermal-displacement distribution with $\boldsymbol{U}=\left\langle\boldsymbol{u} \boldsymbol{u}^{t}>\right.$ being the mean square displacement tensor (ADP). This model describes the density quite well, but only on the atomic position. The electron density between the atoms i.e. in a chemical bond is ignored. This leads to systematic errors in the description of the position of hydrogen atoms. ${ }^{[12-13]}$ With this model, only information about bond lengths and angles is attainable. Information about the properties of a chemical bond is not available from the independent atom model.

\subsection{Multipole Electron Density Model}

A more general description of the density, in the chemical bond as well as at the atomic positions, is achieved by using the multipole model. This model uses X-ray scattering factors which include a full set of spherical harmonics. The density model introduced by Stewart ${ }^{[14-15]}$ and first implemented in software (MOLLY, LINEX74) by Hansen and Coppens ${ }^{[16-17]}$ consists of a superposition of harmonically vibrating aspherical atomic density distributions. Each atomic density is described as a series expansion in real spherical harmonic functions usually up to and including fourth-order $(\mathrm{l}=4)$.

$$
\rho_{\text {atomic }}(\boldsymbol{r})=P_{c} \rho_{c}+P_{v} \kappa^{3} \rho_{v}(\kappa r)+\sum_{l=0}^{4} \kappa^{\prime 3} R_{l}\left(\kappa^{\prime} \boldsymbol{r}\right) \sum_{m=-l}^{l} P_{l m} Y_{l m}(\vartheta, \varphi)
$$

The atomic electron density $\rho(\boldsymbol{r})$ is divided into three components where $P_{c}$ and $P_{v}$ are the spherical core and valence densities, respectively, and the summation of $P_{l m}$ in the third term accounts for valence deformations. The $Y_{l m}$ are density-normalized real spherical harmonics expressed in polar coordinates. The valence density $\rho_{v}$ and the radial functions $R_{l}$ are modified by the scaling parameters $\kappa$ and $\kappa^{\prime}$, respectively, to account for the radial expansion and contraction of the valence shell. The total number of electrons associated with one atom is equal to $P_{c}+P_{v}+$ $P_{00}$ since the higher terms with $l \neq 0$ integrate to zero when integration is performed over space. The functions $\rho_{\text {core }}$ and $\rho_{\text {valence }}$ are chosen as Hartree-Fock densities of the free atoms normalized to one electron. The radial functions $R_{l}(\boldsymbol{r})$ are taken as simple Slater functions calculated for free atoms with energy-optimized exponents $\left(\zeta_{l}\right)$. The exponents $n_{l}$ account for deformation in the valence density.

$$
R_{l}(\boldsymbol{r})=\frac{\zeta_{l}^{n_{l}+3}}{\left(n_{l}+2\right) !} r^{n_{l}} e^{-\zeta_{l} r}
$$


In contrast to the simple IAM model where each atoms is described by nine parameters (three coordinates and six anisotropic displacement parameters), the number of parameters in the case of $l=4$ can be up to 35 or even more with anharmonic motion. To tackle this problem, several procedures are commonly used. The $\kappa^{\prime}$-parameters are usually constrained to be the same value. $\kappa$ and $\kappa^{\prime}$ are usually not refined together because they correlate strongly. Atoms can be constrained to share a set of radial expansion functions if the molecule is symmetric and atoms chemically equivalent. The number of population coefficients can be further reduced by applying local symmetry. For example if an atom has local mirror symmetry, only the radial expansion functions which follow this symmetry are refined. To define this symmetry, the orientation of the spherical harmonic functions in space is defined by a local orthogonal coordinate system for each individual atom. By using this approach, the local coordinate system can then be directed to a setting that is convenient for the representation of the local symmetry.

\subsection{Electron Density Transfer from Databases}

Common solutions for the inherent weakness of the multipole model, i.e. that it needs so many parameters, are databases with pre-defined parameters. These databases provide aspherical atomic form factors. The principle of these databases is that pre-defined multipole parameters are transferred to the model and either kept as they are, or they are used as an improved starting model. When the multipole parameters from the database are not refined, the model needs only the amount of parameters of a regular IAM refinement but has the advantage of the aspherical description of the electron density. Two different types of databases exist at the moment: (1) Databases with atomic form factors from quantum mechanical calculations and (2) those with multipole populations generated from previous experimental multipole models. The first category of databases consists of the invariom (transfer invariant atoms) database ${ }^{[18-22]}$ and the UBDB (University of Buffalo Databank) ${ }^{[23-26]}$. In the second category are the ELMAM (experimental library multipolar atom model) ${ }^{[27-28]}$ and the SBFA (Supra molecular Synthon Based Fragments Approach $)^{[29]}$. All such database approaches assume that a molecule can be divided into electron density fragments which, to a good approximation, remain unchanged in various molecules. The density fragments can be compared to the synthon in retrosynthetic chemistry. ${ }^{[30]}$ By transferring of the aspherical atom form factors to a target-molecule the molecular electron density is reconstructed from atomic electron density fragments. The practical aspects of the invariom database are explained in chapter 4.4.2.

\subsection{Topological Analysis of the Electron Density}

In the early charge density literature the properties of the density was analyzed with static deformation density maps. ${ }^{[31]}$ The static density deformation density is given as

$$
\Delta \rho(\boldsymbol{r})_{\text {stat. }}=\rho(\boldsymbol{r})-\rho_{\text {stat. }}(\boldsymbol{r})=\rho(\boldsymbol{r})-\sum_{j=1}^{N} \rho\left(\boldsymbol{R}_{j}\right)
$$

with $N$ being the number of valence electrons of the corresponding atom $j$ and $\boldsymbol{R}_{j}$ the position of the respective atom $j$. These maps are based on the functions and populations of the asphericalatom multipole refinement and represent the aspherical electron density change by reference to the Independent Atom model. The deformation density maps provided the first confirmation that 
bonding features are accessible by X-ray methods and thus confirmed long-used chemical concepts, but for deeper analysis of the density this method was not sufficient. ${ }^{[32]}$

\subsubsection{Quantum Theory of Atoms in Molecules}

The result of a multipole refinement against high-resolution X-ray data is the atomic distances and angles in the crystal as well as the distribution of the electron density. The multipole model as such improves the positional and vibrational parameters in comparison with the IAM model, but for this improved structural data alone the experimental effort would be too great in most cases. ${ }^{[16]} \mathrm{A}$ lot more additional information about the molecule in a crystal can be obtained from the distribution of the electron density. For this purpose, Richard F. W. Bader developed the Quantum theory of Atoms in Molecules (QTAIM), based on the topology of the total electron density, which leads to classification of the molecular properties. ${ }^{[33-38]}$ Beside other features, the electron density is analyzed for two major characteristics - the existence of critical points and bond paths. ${ }^{[39]}$ The nuclear-electron attractive force in a molecule and the form of charge distribution is the result of the balance achieved in the competition of the nuclei in the system for its charge density. The major consequence of the dominance of this force is that $\rho$ is a local maximum only at the position of a nucleus. ${ }^{[40]}$ The interaction between a pair of atoms thus results in the formation of a line of maximum density linking the nuclei of the two atoms and forms a surface defining their mutual boundary which intersects this line at the point where the density attains it minimum value. This line is called a bond path. The point on the bond path where the boundaries of two atoms connect is called a critical point.

\subsubsection{Critical Points}

Critical points in the density (minima, maxima and saddle points) are located at points for which the gradient of the density is zero.

$$
\nabla \rho(\boldsymbol{r})=\left(\begin{array}{l}
\frac{\partial \rho}{\partial x} \\
\frac{\partial \rho}{\partial y} \\
\frac{\partial \rho}{\partial z}
\end{array}\right)=0
$$

The sign of its second derivatives or curvature determines whether an extremum is a minimum or a maximum. In three-dimensional space for a given set of coordinate axes and the eigenvalues, which are also denoted the curvatures are determined by the Hessian matrix or Hessian of $\rho$ :

$$
H(\boldsymbol{r})=\left(\begin{array}{ccc}
\frac{\partial^{2} \rho}{\partial x^{2}} & \frac{\partial^{2} \rho}{\partial x \partial y} & \frac{\partial^{2} \rho}{\partial x \partial z} \\
\frac{\partial^{2} \rho}{\partial y \partial x} & \frac{\partial^{2} \rho}{\partial y^{2}} & \frac{\partial^{2} \rho}{\partial y \partial z} \\
\frac{\partial^{2} \rho}{\partial z \partial x} & \frac{\partial^{2} \rho}{\partial z \partial y} & \frac{\partial^{2} \rho}{\partial z^{2}}
\end{array}\right)
$$

Diagonalization of the Hessian matrix yields the principal axis and the eigenvalues, which are also denoted the curvatures of the matrix (often just called $\lambda_{n}$ ). The Laplacian of the density is given by the sum of the diagonal elements. These diagonal elements are often just called $\lambda_{n}$. 


$$
\nabla^{2} \rho(\boldsymbol{r})=L(\boldsymbol{r})=\frac{\partial^{2} \rho}{\partial x^{2}}+\frac{\partial^{2} \rho}{\partial y^{2}}+\frac{\partial^{2} \rho}{\partial z^{2}}=\lambda_{1}+\lambda_{2}+\lambda_{3}
$$

While all of the eigenvalues of $\nabla^{2} \rho(\boldsymbol{r})$ at a critical point are real, they may equal zero. The rank of a critical point, denoted by $\omega$ is equal to the number of non-zero eigenvalues of $H(\boldsymbol{r})$ at the critical point. The signature $\sigma$ is simply the algebraic sum of the signs of the eigenvalues at the critical point. The critical point is labeled by giving the duo of values $(\sigma, \omega)$. Generally, for molecules the critical points are all of rank 3 . For example the critical point of an ordinary $\mathrm{C}-\mathrm{C} \sigma$-bond has the signature $(3,-1) .^{[36,41]}$

$(3,-3) \quad$ All curvatures are negative and $\rho$ is a local maximum at the critical point. This point is usually found at the atom position.

$(3,-1)$ Two curvatures are negative and $\rho$ is a maximum in the plane containing the two negative curvatures and minimum along the perpendicular bond path (saddle point). These points are found in covalent bonds.

$(3,+1)$ Two curvatures are positive and $\rho$ is a minimum in the plane containing the two positive curvatures and maximum in the perpendicular direction (saddle point). This type of critical point is found at the center of a ring formed by $n$ atoms.

$(3,+3)$ All curvatures are positive and $\rho$ is a local minimum at this point. This type of point is usually found in the center of a cage.

When the Laplacian $\nabla^{2} \rho(\boldsymbol{r})$ is negative, the electron density is locally concentrated at the bond critical point (BCP), which in turn exerts a net attractive force on the nuclei of the bonded atoms. This can be used to distinguish between various types of interactions. The high electron density at the BCP for negative values of $\nabla^{2} \rho(\boldsymbol{r})$ is commonly associated with a covalent character of the bond (shared interaction), while distinct positive values of $\nabla^{2} \rho(\boldsymbol{r})$ in connection with low electron density at the BCP are attributed to closed shell interactions (e.g. ionic, coordinative, metal-metal bonds). ${ }^{[41,10,42-44]}$ The characterization of bonds by the sign of the Laplacian at the BCP is not always unambiguous. Especially in weak bonds with low density accumulation between the bonded atoms and in bonds where the distribution of $\nabla^{2} \rho(\boldsymbol{r})$ along the bond path has a high slope at the BCP the value of $\nabla^{2} \rho(\boldsymbol{r})$ alone can lead to misinterpretations. The values of the BCPs have always to be seen in the context of their chemical environment.

Chemical bonds can be divided in two major classifications: shared interactions: $\nabla^{2} \rho(\mathbf{r})<0$; the charge density is contracted along the bond path, which leads to a negative Laplacian and increased $\rho\left(\mathbf{r}_{\mathrm{BCP}}\right)$, respectively.

closed shell interactions: $\nabla^{2} \rho(\boldsymbol{r})>0$; internuclear depletion of the charge density and accumulation in the regions of the valence densities are observed, which leads to a positive Laplacian (positive eigenvalue $\lambda_{3}$ dominates) and low values of $\rho(\mathbf{r})$ at the BCP, respectively.

Instead of searching for critical points in $\rho(\boldsymbol{r})$ it is also possible to search for critical points in $\nabla^{2} \rho(\boldsymbol{r})$. The sign of the Laplacian displays whether a charge concentration $\left(\nabla^{2} \rho(\boldsymbol{r})<0\right)$ or depletion $\left(\nabla^{2} \rho(\boldsymbol{r})>0\right)$ is present. Maxima in the negative Laplacian, $(3,-3)$ critical points in $\nabla^{2} \rho(\boldsymbol{r})$, are therefore indicative of local charge concentrations, called valence shell charge concentrations (VSCCs). These concentrations often indicate bonding electron pairs or non-bonding charge concentrations (lone-pairs). ${ }^{[45]}$ 


\subsubsection{Ellipticity}

Additional information can be gained by the value of the bond ellipticity ${ }^{[46]}{ }^{[40,47]}$ where the two $\lambda$ values are perpendicular to the bond vector (Figure 1).

$$
\epsilon\left(\boldsymbol{r}_{B C P}\right)=\left(\left|\lambda_{1}\right| /\left|\lambda_{2}\right|-1\right)
$$

$\epsilon$ quantifies the deviation from rotational symmetry for a given bond density distribution. The value of $\epsilon$ is zero for the $C-C$ bond of order 1 in ethane and attains some maximum value for $C=C$ $\pi$-bonds of higher order. The ellipticity thus provides a quantitative measure of the $\pi$-character of these bonds and the plane of the $\pi$-distribution is uniquely specified by the direction of the axis associated with the curvature of the smallest magnitude, $\lambda_{2}$.

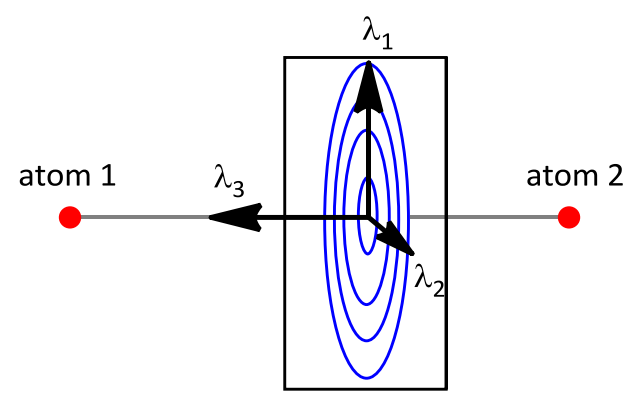

Figure 1 Spatial orientation of the eigenvalues $\lambda_{i}$.

Another parameter to classify the type of a bond is the ratio $\eta\left(\boldsymbol{r}_{B C P}\right)=\left|\lambda_{1}\right| / \lambda_{3}$. It is the ratio of the $\lambda_{1}$ and the curvature along the bond path. The value of $\eta\left(\boldsymbol{r}_{B C P}\right)$ is $<1$ for closed shell (ionic) interactions, increases with bond strength and decreases with the ionic contribution in shared (covalent) interactions. It decreases, for example, in the sequence ethylene (4.31), benzene (2.64), ethane (1.63)..$^{[41]}$

\subsubsection{Atomic Basins}

The topology of the total density allows a different definition of an atom in a molecule by dividing the density into atomic basins (Figure 2). Inside each basin the atomic electron density may be integrated yielding the topological charge (sometimes called Bader charge). The surface of the atomic basins is called the zero-flux surface (ZFS).

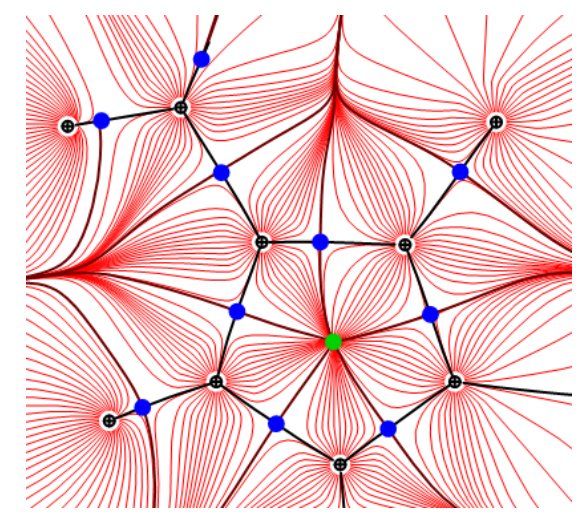

Figure 2 Gradient vector plot (blue: bond critical point; green: ring critical point; brown line: zero-flux surface) 
The normal vector $\boldsymbol{n}$ of the ZFS stays perpendicular on the gradient of the electron density at all points on the surface. Through the course of the trajectories of the gradient vector field it is possible to partition a molecule into its atomic basins $\Omega$.

$$
\Delta \rho(\boldsymbol{r}) \cdot \boldsymbol{n}(\boldsymbol{r})=0 \text { for all points on the ZFS. }
$$

Integration of the electron density over the volume of an atomic basin results in the number of electrons $N(\Omega)$ of an atom and $N(\Omega)$ minus the atomic number gives the atomic charge $Q(\Omega)$.

$$
\begin{gathered}
V(\Omega)=\int_{\Omega} d \tau \\
N(\Omega)=\int_{\Omega} \rho(\boldsymbol{r}) d \tau
\end{gathered}
$$

Figure 2 shows the gradient field of a heterocyclic ring. The atomic basins are separated by the brown lines which represent the border of the zero-flux surfaces. Integration of the atomic density sums up the density inside these borders for each atom. A minimum in the density is located in the center of the ring in form a ring critical point.

\subsubsection{Residual Density Analysis}

In X-ray diffraction experiments aiming at the reconstruction of the electron density in the unit cell, the parameters describing the electron density are obtained by a minimization of the difference between $F_{o b s}^{2}$ and $F_{c a l c}^{2}$. The resulting least-squares fit is judged by the 'flatness' and the 'featurelessness' of the residual density. With the relatively new program jnk2RDA by Meindl and Henn, the residual density of an X-ray experiment can be analyzed for several quality indicators. ${ }^{[48]}$

$$
e_{\text {net }}=\frac{V}{N} \sum_{k=1}^{N} \rho_{0}(k) \quad \rho_{\text {net }}=\frac{1}{N} \sum_{k=1}^{N} \rho_{0}(k)
$$

The number of net residual electrons $e_{n e t}$ is defined as the excess or missing number of electrons in a given volume of the unit cell. The summation over the grid points of a residual density grid of the unit cell gives the net residual density $\rho_{\text {net }}$.

$$
\rho_{\text {gross }}=\frac{1}{2 N} \sum_{k=1}^{N}\left|\rho_{0}(k)\right|
$$

To quantify the absolute value of residual density $\rho_{\text {gross }}$ gives an average residual density in the unit cell.

$$
d^{f}\left(\rho_{0}=x\right)=\lim _{\varepsilon \rightarrow 0} \frac{\log N(x, \varepsilon)}{\log (1 / \varepsilon)}
$$

To calculate the fractal dimension $d^{f}$ as defined by Bronstein et al. ${ }^{[49]}$ the program covers the unit cell with a grid of the box size $\varepsilon$. When $N(x, \varepsilon)$ is the number of boxes where $x$ is present, then the fractal dimension is $d^{f}$. For a three-dimensional grid with no residual density, the fractal dimension is 3 . When residual density is present, the fractal dimension is lowered. 


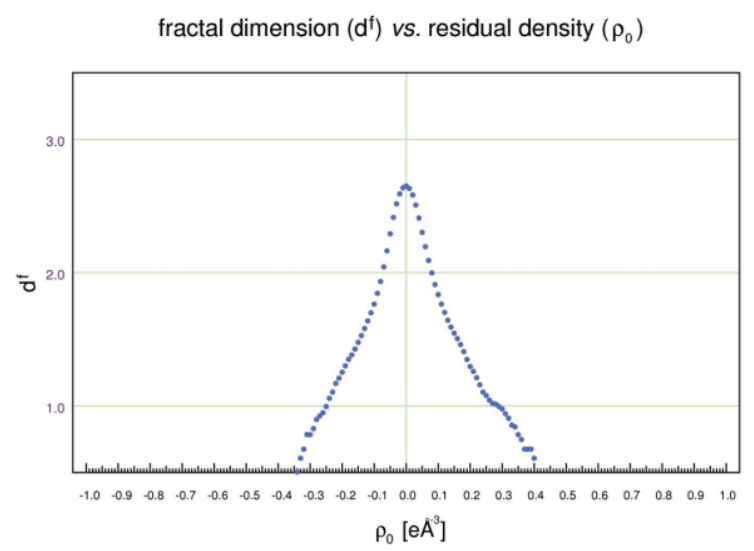

Figure 3 Example of a fractal dimension distribution plot of the residual density. ${ }^{[48]}$

The plot in Figure 3 shows a typical fractal dimension distribution plot as function of the residual density. For experimental data where systematic errors and noise are always present the fractal dimension is below 3 and the distribution curve deviates from the ideal Gaussian shape. The lower the residual density after the refinement, the lower are the absolute values on the abscissa.

\subsection{Scope}

The scope of this work was the analysis of the electron density of low valent tetreles. For silicon and carbon-containing compounds. Especially low valent silicon compounds are of great interest because their synthesis has always been a challenging target. Since the chemistry of aromatic systems with low-valent silicon is a relatively new field, obtaining new information on their fundamental properties is likely to help with the understanding their reactivity and synthetic access.

The investigation of low-valent silicon compounds in terms of experimental electron density analysis has always been difficult. Low-valent silicon compounds are only stable when they are stored at low temperature or kinetically stabilized with sterically demanding ligands. However, such ligands often do not pack well while forming single crystals. Instead, they are almost always disordered and the analysis of the electron density in combination with disorder is difficult. The multipole refinement with X-ray data of small molecules up to about thirty atoms has become a quasi-routine method over the last years. Most common refinement programs focus on this, unfortunately. Much less attention has been paid to the multipole refinement of larger molecules and disorder treatment. The aim of this study was the development of the necessary procedures for the treatment of disorder and perform a complete electron density analysis even if major parts of the molecule were disordered. The Invariom approach was a key tool to model the disordered parts of the structures with aspherical density and also allowed to refine certain parts of a molecule freely.

The scope of the synthesis in chapter 7 was to obtain metal complexes of tripodal iminophosphoranes and to characterize them by single crystal X-ray diffraction. In addition, a similar tripodal 1,2,3-triazole containing ligand system could be established and characterized by single crystal $X$ ray diffraction. The tripodal iminophosphoranes turned out to be very strong neutral ligands for tin(II) and nickel(II) salts. 


\section{Crystallographic Section}

\subsection{Crystal Application}

The selection of air and moisture sensitive crystals was performed in an inert atmosphere using the X-Temp2 ${ }^{[50-51]}$ device on a movable desk with Argon Schlenk-line and a bifocal microscope. Crystals were picked from their mother liquor in a Schlenk-flask under slight argon stream with a spoon or needle and dispersed in perfluorinated polyether oil. The X-Temp2 cooling device allows cooling the oil under the microscope from $25^{\circ} \mathrm{C}$ to a temperature of $-100{ }^{\circ} \mathrm{C}$ by using a nitrogen gas stream. The cold gas stream on the one hand protects the sample from air and moisture, and on the other hand drastically reduces the kinetics of diffusion and decomposition in the protective environment of the inert oil. Suitable crystals were selected using the polarization filter of the microscope, and were positioned on the tip of a glass fiber or MiTeGen Loop. The sample was then quickly positioned in the cold inert gas stream of the low temperature device installed on the diffractometer. This way, the oil around the crystal was shock-cooled forming a glass.

\subsection{Data Collection}

\subsubsection{Data Collection for Routine Structures}

The data for routine measurements were collected on Bruker TXS, Incoatec Mo and Ag I $\mu S^{[52]}$ and Cu-smart 6000 diffractometers. To gain full completeness and enough redundancy of the whole reciprocal space in the shortest possible time the data collection strategy was usually calculated with COSMO included in the APEX2 software suite. When possible, a video of the crystal was taken to determine the crystal faces for absorption correction and size measurements. All crystals were cooled with a Bruker Kryoflex I or II device and with an Oxford Cryosystems Cryostream on the smart 6000 diffractometer (in most cases $100 \mathrm{~K}$ ) during the measurement.

\subsubsection{High Resolution Data Collection}

For experiments analyzing the electron density after a multipole refinement, it is essential to collect data of the highest possible quality. In contrast to a routine measurement the following important extra procedures have to be performed:

- The selected crystal must be of superior quality! Crystals of quality just sufficiently enough for a routine (IAM) structure refinement are not suitable. The crystal has to be absolutely clear, without cracks, has to have clearly defined faces and as small as possible to scatter to a sufficient resolution but, if possible, not bigger than the beam diameter. ${ }^{[52]}$ Crystals can often be cut with a sharp scalpel. It is usually beneficial to cut parallel to the crystal faces. Some crystals get damaged when cutting. Those have to by crystallized in the desired size. The crystal should show no extra reflections from separate (adherent) satellite crystals after the unit cell determination.

- The correct mounting of the crystal is important for a routine measurement but is much more essential for electron density experiments. The mounting material has to withstand the forces of gravity and the cold gas stream but its contribution to background scattering must be as low as possible. This also means that small quantities as possible of the coating oil should be used. 
Several possibilities exist to mount single crystals on a diffractometers goniometer head. The traditional way with capillaries has the major drawback that the glass has a strong background scattering and the relatively thick and long capillary disturbs the laminar gas stream. The stream gets turbulent around the capillary and the nitrogen gas mixes with ambient air. This results in ice formation at the border between the turbulent cold gas stream and the ambient air. A better alternative are synthetic loops. Inside the loop, a single crystal can be held in a glass of coating oil. Loops also have the tendency to incorporate too much oil and thus produce background scattering. Their advantage is that the handling of sensitive crystals is quicker and easier. The least background scattering is to be expected from crystals mounted on glass fibers and on MiTeGen loops. MiTeGen loops are made of kapton which makes these loops mechanically very robust. Some practice is needed to handle glass fibers but their interference with the cold gas stream is ideal. MiTeGen loops, in contrast, have a slightly bigger tendency of icing but they are very easy to handle. Natural products like cat whiskers and cactus needles are not well suited, because they are not always inert to the crystal, and they can have holes in which crystals can vanish.

- The resolution of the experiment should cover the full reciprocal space up to the highest possible resolution and should exceed $0.45 \AA\left(\sin \theta / \lambda=1.11 \AA^{-1}\right) .^{[10]}$

- The multiplicity should be much higher than for routine measurements. Up to a resolution of about $0.6 \AA$ the overall multiplicity should be about 15 and up to $0.45 \AA$ must be at least three with an $I / \sigma(I)$ higher than three. The strongest reflections at low resolution must be measured with special care! In these reflections relies the most information about diffuse density. Especially strong reflections which are measured in the shadow of things like the beam stop or the crystal cooling device can be measured systematically wrong. But also individual strong reflexes may be absent because they are overexposed.

- Attention should also be paid on the technical status of the diffractometer before the measurement starts. Even with a high-flux rotating anode, the measurements can last two weeks for a triclinic crystal. During this time, technical errors may occur, which would destroy the measurements. For example, the cooling should work perfectly, the cross hair of the microscope camera should be aligned (as in chapter 10.1), and the flux of the X-ray beam as well as its alignment should be checked.

- The crystal temperature should be as low as possible to obtain a good deconvolution of thermal motion and the static electron density on the one hand and stronger scattering due to less thermal movement of the atoms on the other hand. ${ }^{[10]}$ Low temperature also minimizes the contribution of thermal diffuse scattering (TDS) to the measured intensity. The contribution of TDS can be up to $20 \%$ for high angle data at $100 \mathrm{~K}$. Because the TDS behavior is a property of the particular crystal only few things can be done to minimize this effect. One is lowering the temperature and there are methods to correct for it empirically. ${ }^{[33-59]}$

- The contribution of the scattering from the atomic core density increases with higher atomic number. This implicates that the data at low angles has to be measured with special care for heavier elements to not saturate the detector. Modern CCD detectors have a dynamic range of $\sim 10^{5} .{ }^{[60]}$ In combination with modern high brilliant $X$-ray optics and sources, this makes it difficult to collect precise low angle data without overflows.

- Modern software for data collection mostly has advanced tools for the calculation of a desired measurement strategy. Especially for electron density measurements, the manual measurement 
of omega scans with $90^{\circ}$ or $45^{\circ}$ interval in phi is not sufficient. The number of reflections increases with $(\sin \theta / \lambda)^{4}$. This makes it difficult to measure full completeness at high resolution without the real knowledge of where the reflections are to be expected. Strategy software knows where it can expect the reflections and thus enables to measure full completeness at full resolution. Moreover, the redundancy of the data should be planned before the measurement. The SADABS (and other) software needs equivalents to correctly account for scaling and absorption. The more equivalents the better.

\subsubsection{Judgment Criteria for Data Quality}

The quality of the data, which are necessary for a routine structure refinement is relatively well defined. ${ }^{[61]}$ The criteria for the data quality of electron density refinements are much harder to define. The requirements for much higher resolution and zero residual density, in the ideal case, do not forgive any weaknesses in the data quality. Therefore, the data for electron density investigations have to be processed with special care. All steps from integration, scaling, absorption correction, and evaluation of the data quality are not routine tasks and must be customized for every measurement.

The data reduction (integration) of the Bruker frames were done with SAINT ${ }^{[62]}$ using a mixed method of 3D-profile fitting by Kabsch ${ }^{[63]}$ for $I / \sigma(I)<10$ and summation of intensities for strong reflections $(I / \sigma(I)>10)$. The main function of SAINT is to reduce one or more runs of sequential frames, acquired with the APEX II and BIS software, to $h k l, I, \sigma(I)$, and related information for each reflection. Additionally it performs an incident angle correction ${ }^{[64-65]}$ and corrects for Lorentz polarization. ${ }^{[66]}$ The data for electron density refinements have to be integrated with special care. Since the X-ray experiment has no absolute defined outcome, no precise definition for of good data quality exists. But the internal R-value and other data statistics will hint if data are good or not. The integration program needs the unit cell data and the orientation of the crystal in 3D-space relative to the goniometer geometry to know where it can expect reflections on the measured data frames. The integration program finds the unit cell (CELL, CELLSD) and the orientation matrix (ORT1-3) in the $p 4 p$ file:

\begin{tabular}{|c|c|c|c|c|c|c|c|}
\hline CELL & 21.5066 & 17.3759 & 25.9784 & 90.0000 & 94.5734 & 90.0000 & 9677.162 \\
\hline CELLSD & 0.0007 & 0.0005 & 0.0008 & 0.0000 & 0.0013 & 0.0000 & 0.641 \\
\hline ORT1 & -1.65384 & $e-002$ & $-5.2047316 e-002$ & -1.02 & $3803 e-002$ & & \\
\hline ORT2 & -2.38754 & $e-002$ & $2.3036567 e-002$ & -3.08 & $5900 e-002$ & & \\
\hline ORT3 & 3.650032 & $e-002$ & $-8.5142534 e-003$ & -2.08 & $2213 e-002$ & & \\
\hline
\end{tabular}

The initial orientation is then refined after a certain number of frames to compensate for small movements of the crystal. The small movements are mainly caused by misalignments of the goniometer or inaccuracy in the centering of the crystal. An indicator to see if the orientation matrix refinement worked well is a Diederichs plot (Figure 4). ${ }^{[67]}$ 

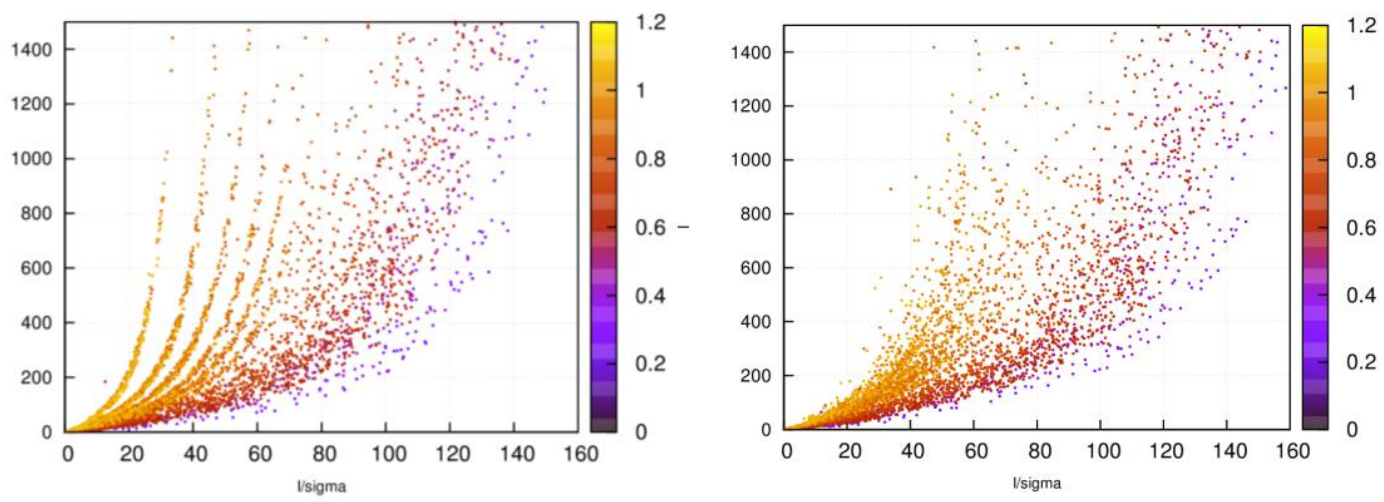

Figure 4 Plot of the intensity $(I)$ versus $(I) / \sigma(I)$ after correction of the respective orientation matrix in several measurement runs. The color represents the resolution in $\sin (\theta) / \lambda$. Left: before the correction; Right: after the correction.

Diederichs used this plot in his publication as indicator for machine errors like misaligned X-ray beam, shutter, goniometer, detector, and also for errors in the exposure time and spindle speed. ${ }^{[67]}$ Furthermore, this plot can also be used to detect movements of a crystal caused by deicing during the measurement. The plot on the left side of Figure 4 is the result of the measurement of structure 1 where the crystal has moved due to deicing. Reintegration of the data with a single orientation matrix for every single run resulted in the intensity distribution in Figure 4 right. The shape of the plot is a result from the estimation of the error $\sigma(a b s)^{2}$ of the measured intensities by the scaling program (SADABS in this work):

$$
\sigma(a b s)^{2}=k\left[\sigma(r a w)^{2}+\left(g\left\langle I_{c}\right\rangle\right)^{2}\right]
$$

where $k$ is a scaling factor to scale the different batches to each other, $\sigma(a b s)$ the corrected standard uncertainty of the corrected intensity $I_{c}$, and $\sigma(\mathrm{raw})$ is the raw error from the integration program. SABDABS tries to find a value for $\mathrm{g}$ and $\mathrm{k}$ where the best fit between model and experiment is achieved. The formula used to estimate $\sigma(a b s)^{2}$ by SADABS and some other scaling programs tends to be dominated by the term $g I^{2}$ for very high intensities. This imposes an upper limit on $I / \sigma(I)$ because $I$ cannot be infinitive and $g$ is always larger than zero. Therefore, the $I / \sigma(I)$ shows the limit of the signal-to-noise ratio of the strongest reflections and also highlights if systematic errors in the dataset are present.

Another important quality criterion is the plotted output from SADABS. If the completion and redundancy is too low in a distinct resolution shell, plots of $R_{\text {int }}$ and $R_{\sigma}$ against resolution will increase in this area. The plot should rise steadily. Especially when the data measurement strategy was calculated with COSMO in the APEX I/ suite data areas with low redundancy can happen because it tends to collect the data of medium resolution with too low intensity and multiplicity. The result would be a sawtooth shape in $R_{\text {int }}$ over the resolution range in the SADABS diagnostic plot. 

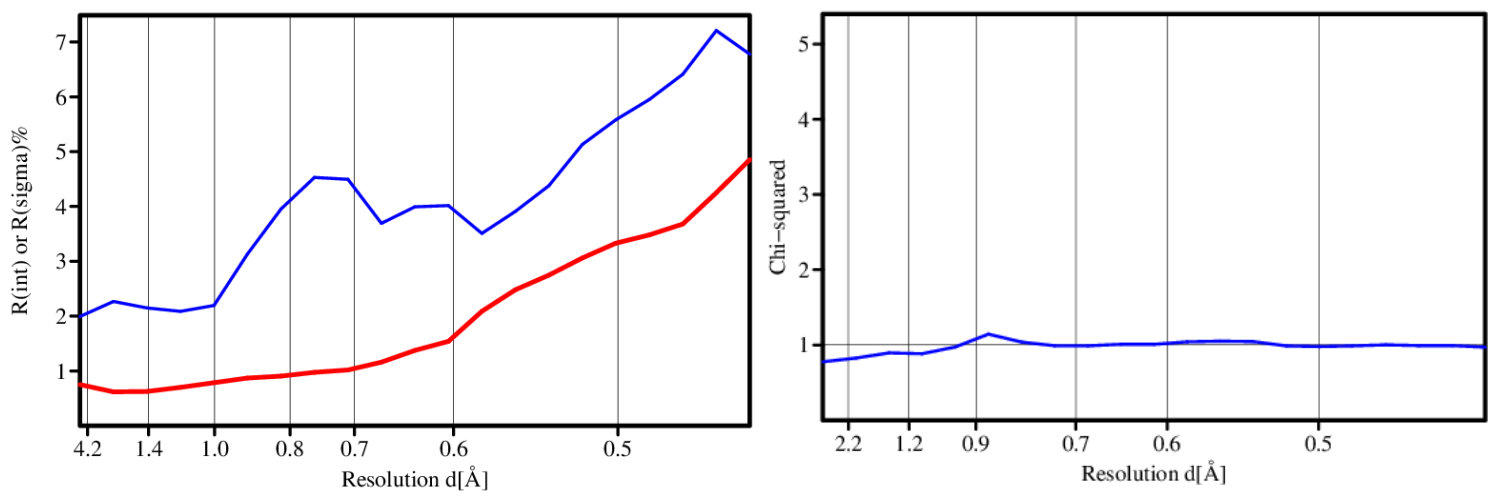

Figure 5 Left: Plot of $R_{\text {int }}$ (blue) and $R_{\sigma}$ (red) against resolution from SADABS. The resolution range from 1.0 to $0.6 \AA$ was measured with too low intensity and multiplicity. Right: Plot of $\chi^{2}$ versus resolution. Deviation from unity at low resolution indicates that there is a problem with the low angle data.

The plot of $\chi^{2}$-values versus resolution should be unity over the full resolution. Based on mean intensities SADABS tries to adjust the parameters $k$ and $g$ in a way such that $\chi^{2}$ is approximately unity for reflection batches.

Additional indicators for the quality of the data can be looked at after a full refinement of the structure. The final $R_{1}$ value, bond length and angle standard uncertainties, and largest peak and hole in the difference electron density map after refinement are very sensitive descriptors of the data quality. But it has always to be clear that the model is correct because a wrong model would also cause bad quality indicators.

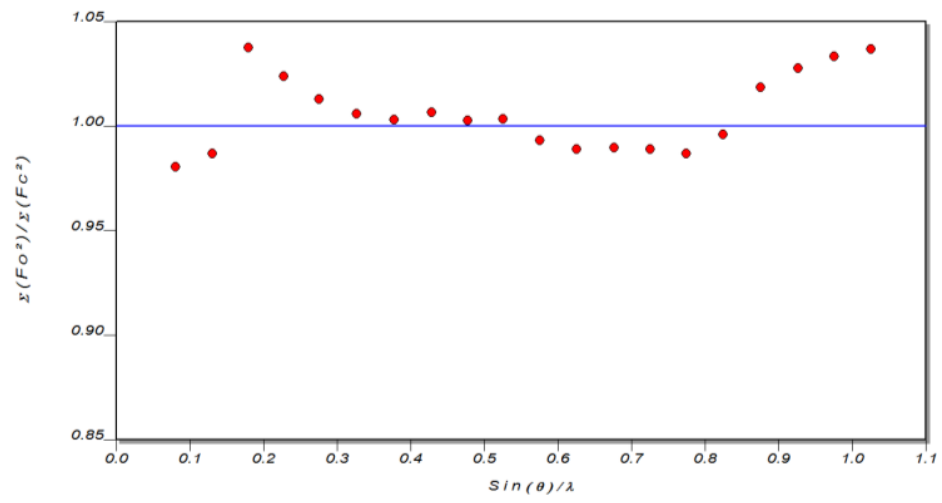

Figure 6 Plot of the scale factor vs. resolution with $d r k p l o t^{[68]}$ after a multipole refinement.

Least squares refinement of $\mathrm{X}$-ray diffraction data against calculated intensities from a (multipole) model is mainly a weighted minimization of $M$ :

$$
M=\sum w\left(F_{o}^{2}-F_{c}^{2}\right)^{2}
$$

$F_{o}^{2}$ and $F_{c}^{2}$ usually depend on the scale factor $k\left(F_{o}^{2}=k F_{c}^{2}\right)$. The variation of the scale factor $k=\sum\left(F_{\text {obs }}^{2}\right) / \sum\left(F_{\text {calc }}^{2}\right)$ in Figure 6 across the entire resolution is an important property in order to assess the success of a refinement. Since one factor scales model and experiment to one another, the scale factor correlates with all parameters. This makes it extremely important that the scale does not change as a function of resolution. The scale factor and the model are always correlated. For example, underestimating of the thermal parameters in the refinement would create a bias which would increase the scale factor at high resolution. Ideal data should allow a refinement with a scale factor of unity over the whole resolution range with minimal residual density. 


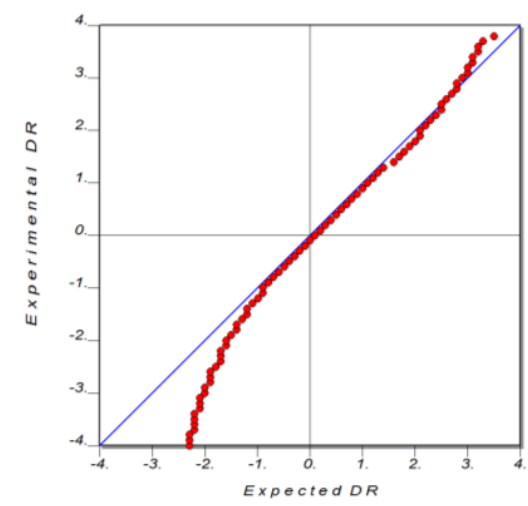

Figure 7 Normal probability plot with experimental vs. expected intensity distribution range.

The calculated and observed intensities of an X-ray experiment should follow a normal distribution. A normal probability plot (Figure 7) shows the agreement between the experimental and the calculated intensities after the refinement. The result can be improved in certain limits with the adjustment of the weighting scheme.

\subsection{Routine Structure Solution and Refinement}

All structures were solved with direct methods using SHELXT ${ }^{[69]}$ It uses the new method where it first solves the phase problem in $P 1$ and then calculates the correct space group from the phases. Afterwards a peak search is performed to find atoms in the density map and simple chemical rules are used to assign element types. The subsequent refinement by full-matrix least-squares methods was performed with SHELXL-12. ${ }^{[70]}$ If not stated otherwise the hydrogen atoms were placed at ideal positions using a riding model where the isotropic motion of the hydrogen atoms at $\mathrm{sp}^{2}$-hybridized atoms were constrained to $120 \%$ and at $s^{3}$-hybridized atoms to $150 \%$ of the $U_{\text {eq }}$-value of their pivot atom.

Structures containing disordered groups were refined using constraints and restraints. While constraints fix structural parameters to certain values, restraints introduce additional chemical or crystallographic information in the model and have to be observed within their standard deviations. In case of a disordered group the occupation of different parts of this fragment was refined with a free variable. If not stated different, the routinely measured data sets from self-crystallized and from cooperation partners were corrected for absorption with semi-empirical methods from equivalents with SADABS 2008/2. ${ }^{[71]}$

\subsection{Electron Density Refinement}

\subsubsection{General Procedure for an Electron Density Starting Model}

The following procedure was used to build a starting model for the electron density multipole refinements: First, the structure was first solved by direct methods and afterwards refined with SHELXL as described in chapter 4.3. This independent atom model served as the starting model for the subsequent multipole refinement. Next the positional and anisotropic displacement parameters of the non-hydrogen atoms were refined using high resolution data only ( $d=0.55-0.00 \AA$ ). During the following refinement steps the resulting positional and anisotropic displacement parameters were kept fixed. The hydrogen atoms were identified by a Difference Fourier Analysis using 
the low resolution data $(d=999-0.98 \AA)$. Then the hydrogen atoms were shifted along their bonding vectors to neutron distances of $1.085 \AA$ for those bound to $\mathrm{sp}^{3}$-hybridized carbon atoms and $1.076 \AA$ for those bound to $\mathrm{sp}^{2}$-hybridized carbon atoms, respectively. ${ }^{[72,13,73]}$

In the case of disordered groups, the 1,2- and 1,3-bond lengths and the thermal displacement parameters from the different parts were restrained to each other. If the positions of hydrogen atoms of disordered parts could not be identified by Difference Fourier Analysis, the hydrogen atoms were placed at ideal positions via a riding model in SHELXL. This has the advantage that the $\mathrm{C}-\mathrm{H}$ distance can simultaneously be set to neutron distances.

\subsubsection{General Procedure for an Invariom Transfer}

The invariom approach was used for the multipole refinement in this work. This allows the description of disorders in the molecule and saves parameters which results in a higher and therefore more reliable data to parameter ratio in the refinement. The invariom approach uses the assumption that aspherical atomic density can be transferred from one molecule fragment to another if the near environment of these atoms is the same. ${ }^{[18-22]}$ For the transfer to be successful, the transfer program (InvariomTool) ${ }^{[21]}$ first has to know the chemical environment of each atom in the target molecule and second the database has to contain the particular atomic density fragments. Additionally, the invariom fragments with their local coordinate systems have to be correctly oriented in space, independently of the molecule or the crystal system. The InvariomTool program analyzes the promolecule for bond orders, ring planarity, ring sizes and the neighbors and next-neighbors of the atoms. With this information InvariomTool searches in the invariom database for the respective fragment and transfers it to the target molecule.

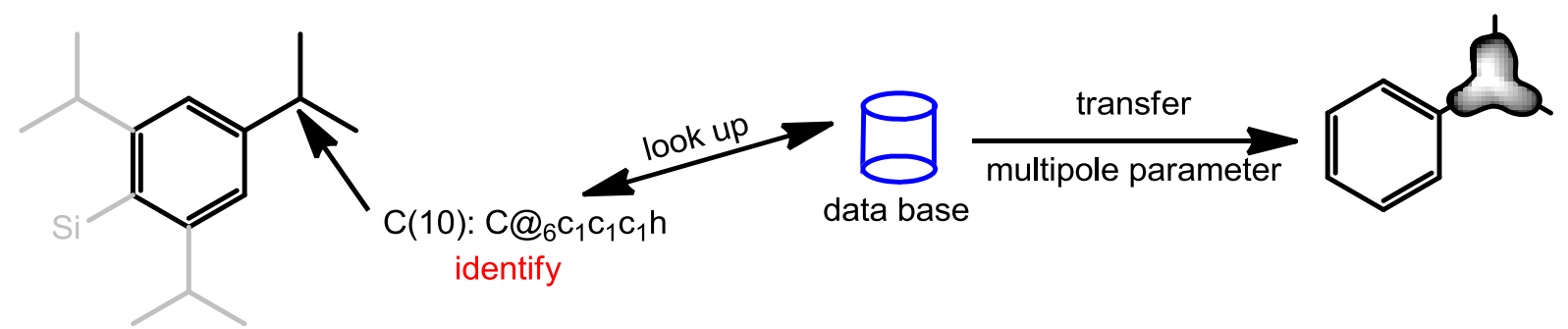

Scheme 1 Schematic representation of an invariom transfer to one atom.

One of the major advantages of the invariom approach is that it is not necessary to calculate the molecular wave functions for a whole molecule. Because of the assumptions made above, it is sufficient to calculate smaller fragments which inherit only the missing invarioms.

A database entry for the tertiary carbon atom in Scheme 1 looks like the following:

Database entry for $\mathrm{C}(10)$ :

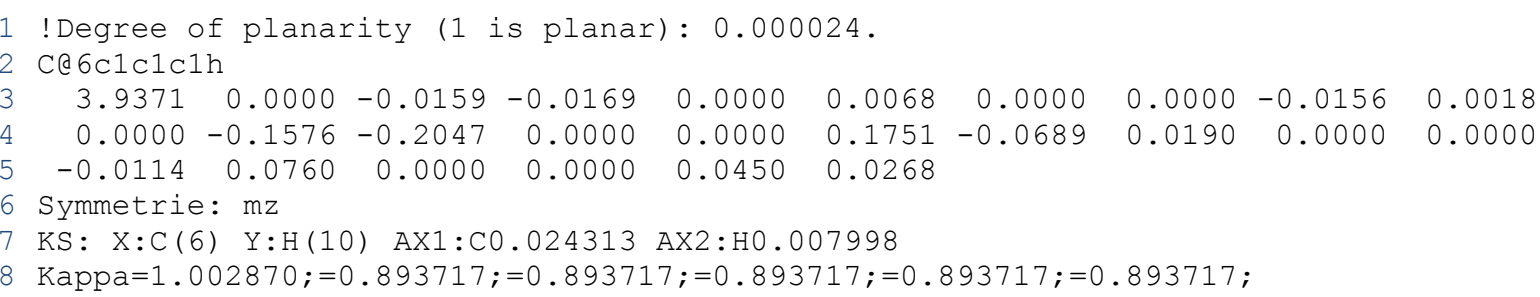

The first line of the example database is the entry for the degree of planarity. Perfect planarity would be the value of one and in this case of tetrahedral environment the value is zero. Line 2 is 
the invariom description of the respected atom. In this case it says "a carbon atom at a six membered ring and two other carbon atoms and one hydrogen atom". The lines 3-5 are the multipole parameters as used in the XD2006 program. Line 6 describes the local symmetry found by InvariomTool. In line 7 are the directions of the axes of the local coordinate system. They are defined by the two shortest bonds ( $\mathrm{XX} 1$ and $\mathrm{AX2}$ ). The last line, the $\kappa$-values are defined.

\subsubsection{XD2006 Refinement Procedure}

The refinement program XD2006 needs three different files as input for the refinement process: $x d$.mas, $x d$.inp and $x d . h k l$. The master file ( $x d$.mas) is the control file which defines the parameters that shall refined. The coordinates, displacement parameter as well as the multipole parameter are fed into XD2006 with the input file xd.inp, and the reflection file xd.hkl. The results are written to the $x d$.res file. And additional listings about the refinement are written to the xdlsm.out file. A summary about bond lengths, angles and multipole parameter can be saved in the xd_geo.out file via xdgeom.

The refinements with XD2006 is performed after building the starting model (chapter 4.4.1) and the invariom-transfer (chapter 4.4.2). To avoid larger correlation between the parameters and to reach better convergence of the least squares fit, the refinements are done in sub-blocks (coordinates, multipoles, displacement parameters, ...) at the beginning. Local symmetry and chemical constraints are applied to every atom to reduce the number of parameters. The general procedure is refining:

1. The scale factor.

2. Dipoles, quadrupoles, octupoles and hexadecapoles of all atoms.

3. Monopoles of all atoms.

4. Coordinates of all non-hydrogen atoms.

5. Anisotropic displacement parameters (non-hydrogen atoms) and isotropic displacement parameters (hydrogen atoms, constrained to the adjacent atom).

6. Monopoles of all atoms, dipoles, quadrupoles, octupoles and hexadecapoles of all atoms.

7. Coordinates of all atoms, displacement parameters of all atoms.

8. RESET of the $\mathrm{C}-\mathrm{H}$ bond lengths to neutron distances and coordinates of all non-hydrogen atoms.

9. Coordinates of all non-hydrogen atoms, monopoles of all atoms, dipoles, quadrupoles, octupoles and hexadecapoles of all atoms.

10. Coordinates of all non-hydrogen atoms, displacement parameters of all atoms, monopoles of all atoms, dipoles, quadrupoles, octupoles and hexadecapoles of all atoms.

11. $\kappa$-parameter.

12. Step 2-10 again.

13. Finally, all parameters together.

For every step the master-, input-, result- and listing files are saved as $x d n$.mas, $x d n . i n p, x d n . r e s$, $x d n \_g e o . o u t(n=01-99)$. This procedure enables the re-refining of the project with small 
corrections or a different $h k l$-file without further user interaction. Therefore, it is possible to compare the quality of different refinement results from various data processing strategies e.g. different integrations or scaling procedures.

\subsubsection{Plotxd - a Tool for Structured Parameter Output from XD2006}

To rearrange the output of $x d g e o m$ from XD2006 in an easy readable way it was necessary to write a specially bespoken script. It also enables plotting of the results along the different refinement steps, which gives a good overview of the refinement procedure. Plotxd assumes that

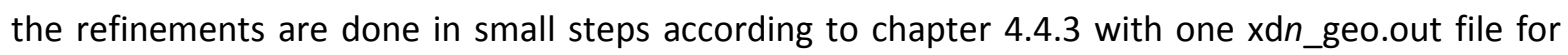
every stage of the refinement $(n=01-99)$. The script creates a directory called "pyout" which contains one text file for every atom of the molecule. Each file includes the following values from each refinement stage: Step-Number, Pval, $\kappa, \kappa^{\prime}$, Net charge, D11+, D11-, D10, Q20, Q21+, Q21-, Q22+, Q22-, O30, O31+, O31-, O32+, O32-, O33+, O33-, H40, H41+, H41-, H42+, H42-, H43+, H43-, H44+, H44-, X, Y, Z, OZ, ISO, U11, U22, U33, U12, U13, U23.

Table 1 Example output of plotxd.py

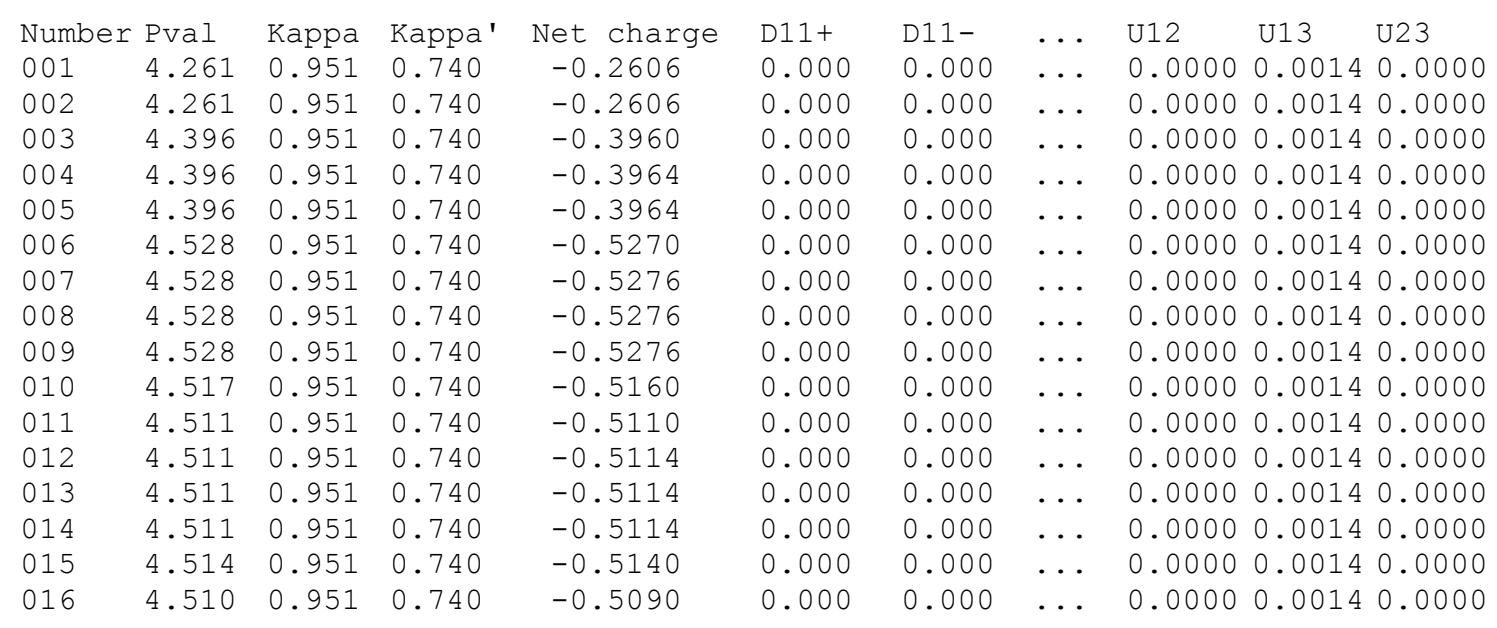

The listings can be written with or without standard deviations as text files (.txt) as well as comma separated values (.csv). The csv-file has the advantage that it can easily be opened by Microsoft Excel. The text file can be plotted with e.g. gnuplot ${ }^{[74]}$ or any other plotting program.

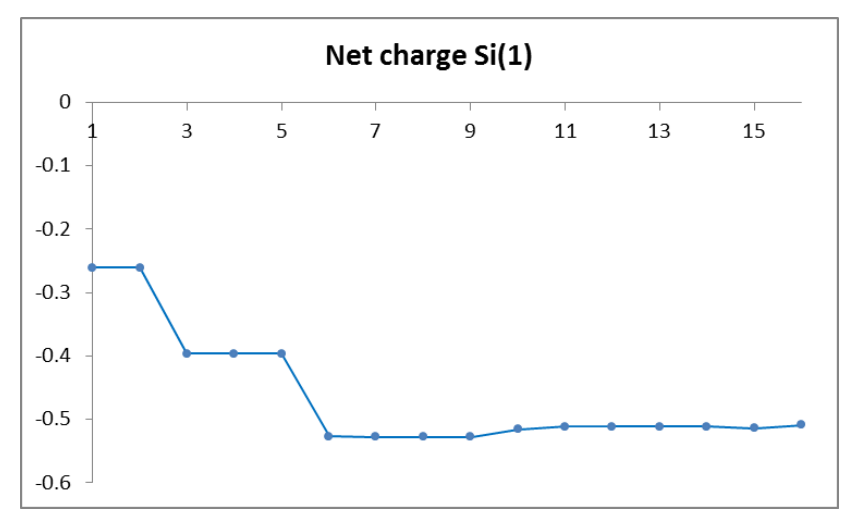

Figure 8 Example plot for the net charge vs. refinement steps for one silicon atom during the refinement. 


\subsubsection{Refcheck - a Refinement Helper Tool}

The refinement of large molecules with XD2006 is not a routine task. For every single atom about 50 parameters have to be correctly adjusted in the master file. For about 200 atoms and 20-30 refinement cycles (chapter 4.4.3 and 5.3) there are thousands of possibilities to generate undetected errors during the refinement process.

A python based program ("refcheck.py") had to be developed to check for the most common errors during the refinement process. The electron density refinements were done in small steps (chapter 4.4.3) with one XD master file for every step in the notation "xd01.mas, xd02.mas, ..." as well as the input files "xd01.inp, $x d 02 . i n p, . .$. ". To give the user the opportunity to check if the user exactly refined the intended items, the refcheck program lists all options which are activated in the master file:

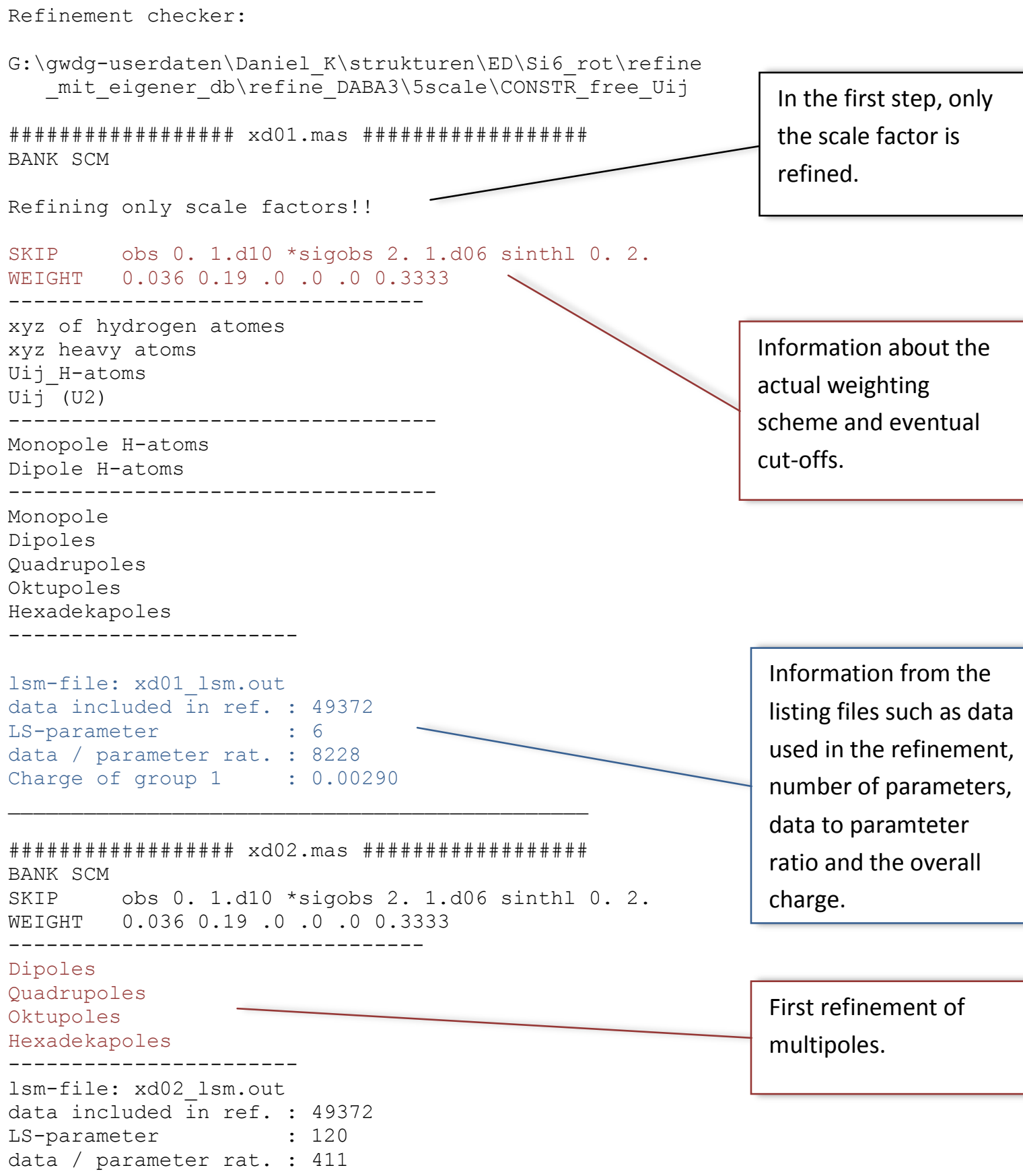
In the first step, only the scale factor is refined.

Information from the listing files such as data used in the refinement, number of parameters, data to paramteter

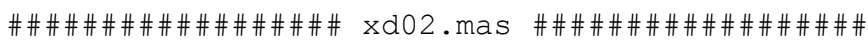
BANK SCM

SKIP obs 0.1.d10 *sigobs 2. 1.006 sinthl 0. 2. WEIGHT $\quad 0.036 \quad 0.19 \quad 0.0 \quad 0 \quad 0.0 \quad 0.3333$ ratio and the overall charge.

Dipoles

Quadrupoles

Oktupoles

First refinement of

Hexadekapoles multipoles.

lsm-file: $x d 02$ lsm.out

data included in ref. : 49372

LS-parameter : 120

data / parameter rat. : 411 


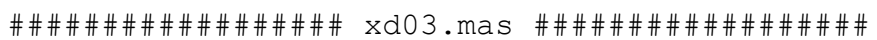

BANK SCM

SKIP obs 0. 1.d10 *sigobs 2. 1.d06 sinthl 0. 2.

WEIGHT $\quad 0.036 \quad 0.19 \quad 0.0 .0 .00 .3333$

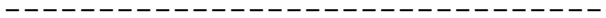

Monopole H-atoms

Monopole

--------------------

lsm-file: xd03_lsm.out

data included in ref. : 49372

LS-parameter : 21

data / parameter rat. : 2351

Charge of group $1: 0.00270$

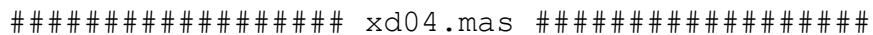

BANK SCM

SKIP obs 0.1.d10 *sigobs 2. 1.066 sinthl 0. 2.

$\begin{array}{lllllllllll}\text { WEIGHT } & 0.036 & 0.19 & 0 & 0 & 0 & 0 & 0 & 0.3333\end{array}$

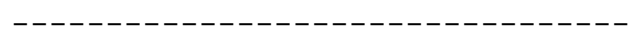

xyz heavy atoms

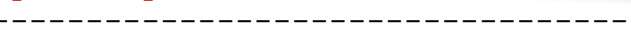

lsm-file: xd04_lsm.out

data included in ref. : 49372

LS-parameter : 149

data / parameter rat. : 331

Charge of group $1: 0.00300$

Refinement of the atomic coordinates.

etc.

Additionally, the refcheck program also checks the consistency of the following conditions and gives a warning if any of these are breached:

- the "title" key in the master file and the hkl-file are same.

- NDAT in hkl-file has the same value as there are rows in the respective hkl-file.

- the $\kappa$ and $\kappa^{\prime}$ values of the hydrogen atoms were left as default.

- the overall charge of the crystal is much bigger than zero or changes drastically during the refinement.

- the numbers of $\kappa$ and the "KEEP kappa" fields are consistent.

- the FOUR field is active, which is useful for residual density maps.

- the refinement of the scale factor is active.

- Extinction is refined.

- RESET is active.

- xyz-constraints are active.

- $\mathrm{U}_{\mathrm{ij}}$-constraints are active.

- coordinates of the non-hydrogen atoms are refined. 
- coordinates of hydrogen atoms are refined.

- $\mathrm{U}_{\mathrm{ij}}$ of the hydrogen atoms are refined.

- monopoles and/or dipoles of the hydrogen atoms are refined.

- $\mathrm{U}_{\mathrm{ij}}(\mathrm{U} 2)$ of the non-hydrogen atoms are refined.

- Gram-Charlier coefficients U3 and/or U4 are refined.

- monopoles, dipoles, quadrupoles octupoles or hexadecapoles of the non-hydrogen atoms are refined.

- $\quad \kappa$ and/or $\kappa^{\prime}$ is refined.

- the data to parameter ratio is too small. 


\section{Experimental Electron Density of Hexasilabenzene}

\subsection{Chemistry of Low Valent Silicon}

The similarities and differences between carbon and its heavier congener silicon still generates challenging synthetic targets. The difficulties in the synthesis of low-valent silicon compounds might be a reason for the formulation of the double bond rule ${ }^{[75]}$ stating that elements after the second row in the periodic table are unable to form $p_{\pi}-p_{\pi}$ bonds under normal conditions. Multiple bonds of this type were exclusively attributed to carbon, nitrogen, oxygen and boron. The double bond rule ignored the fact that even small $\mathrm{p}_{\pi}-\mathrm{p}_{\pi}$ bond containing molecules like $\mathrm{S}=\mathrm{C}=\mathrm{N}^{-}$or $\mathrm{SO}_{2}$ had been known for a long time.
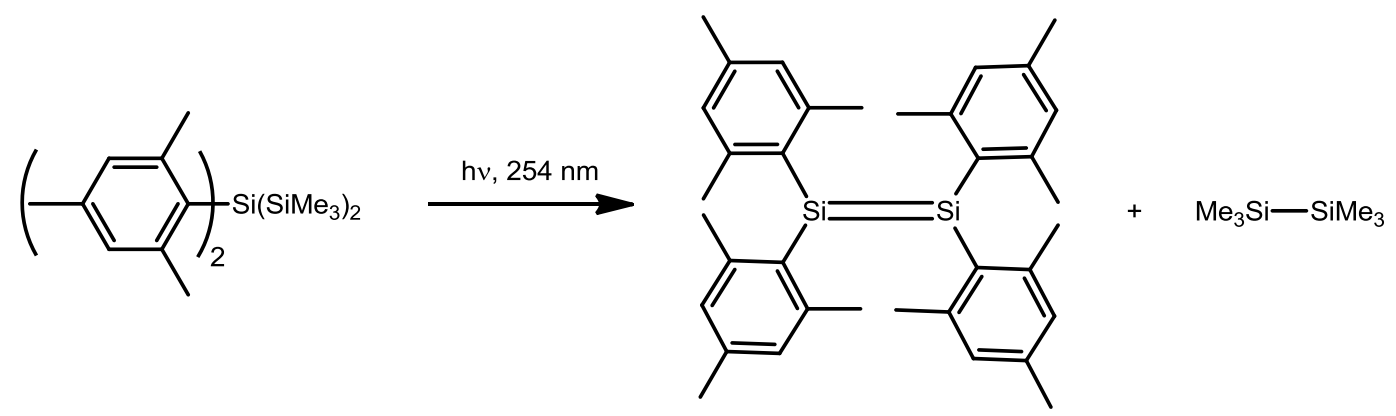

Scheme 2 Synthesis of tetramesityldisilene.

The low valent silicon chemistry started when West and Brook introduced the concept of kinetic stabilization. ${ }^{[76]}$ The first stable compound with a Si=Si double bond goes back to West et al. in 1981 (Scheme 2). ${ }^{[77-78]}$ Apart from their kinetically unstable nature the compounds with Si=Si double bonds are often thermally stable.

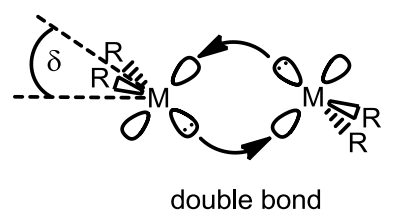

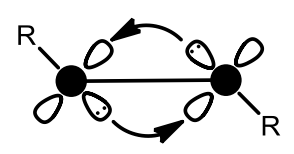

triple bond

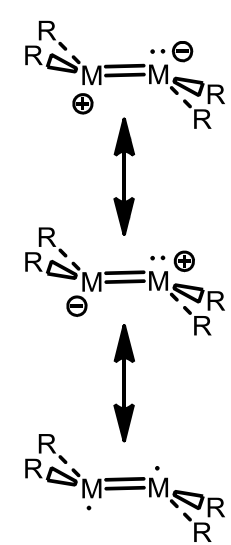

double bond

Scheme 3 Simple bonding models for heavier main group element alkenes ( $M=S i, G e, S n$ or $\mathrm{Pb}$ ). $\delta=$ trans-bent angle.

The nature of the Si=Si double bond was thoroughly investigated over the years. ${ }^{[79-80,78,81-84]}$ The $\mathrm{Si}=\mathrm{Si}$ double bond and its higher homologues show an interesting property ${ }^{[81]}$ In general, molecules with heavier group 14 elements that incorporate multiple bonding are often described as strained geometries that are bent or pyramidalized. This bending is in sharp contrast to the planar or linear 
structure of their carbon-based congeners. Unlike flat-shaped ethylene, structures of heavier main group analogues turned out to be of trans-bent shape (Scheme 3). The left is based on natural localized molecular orbitals and the right on delocalized canonical molecular orbitals. Both have their drawbacks. For instance, a triple bond in the alkyne analogue structure (Scheme 3 left) consist of two donor-acceptor bonds and one $\pi$-bond. If the bending angle is increased to $90^{\circ}$, the donoracceptor bonds are broken and only a $\pi$-bond rather than a $\sigma$-bond remains. The models on the right hand side of Scheme 3 are used for more strongly bent structures. Both models give different bond orders. A different description is based on a second-order Jahn-Teller interaction ${ }^{[85-86]}$ which explains the changes due to a non-degenerate ground state coupled with a low-lying excited state. In molecular orbital terms this becomes an interaction between an occupied high energy $\mathrm{MO}$ and an unoccupied low energy MO. Thus, it is an interaction between HOMO and LUMO in the same molecule. Besides the discussion of the bond origin, the trans-bent angle $\delta$ increases with increasing non-bonded electron pair character from carbon to lead. A very controversial topic is the bond order of multiple bonds because there are no objective parameters to determine the bond order of a chemical bond.
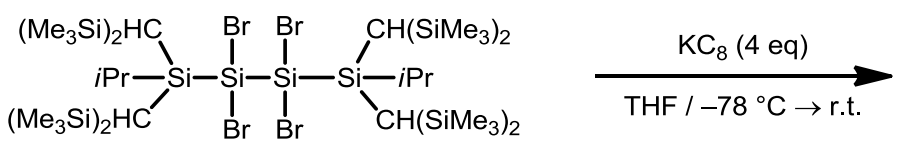

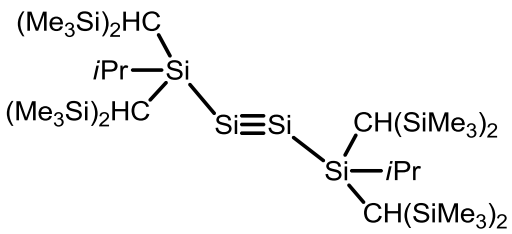

Scheme 4 Disilyne with a silicon-silicon triple bond by Sekiguchi.

The first Si=Si triple bond was synthesized in 2004 by Sekiguchi and Wiberg. ${ }^{[87-89]}$ The Disilyne of Sekiguchi (Scheme 4) was prepared by reduction of the tetrabrominated precursor with potassium graphite. For the success of the synthesis, it was important to use a sterically highly demanding ligand. They used the Dsi ${ }_{2} i \mathrm{PrSi}$ group $\left[\mathrm{Dsi}=\mathrm{CH}\left(\mathrm{SiMe}_{3}\right)_{2}\right]$ for the protection of the $\mathrm{Si}-\mathrm{Si}$ triple bond. The triple bond turned out to be 2.0622(9) $\AA$ long which is $3.8 \%$ shorter than a typical $\mathrm{Si}=\mathrm{Si}$ double bond $(2.14 \AA$ ) and $13.5 \%$ shorter than a single bond $(2.34 \AA)$. In comparison the $\mathrm{C} \equiv \mathrm{C}$ triple bond shows a shortening of only half the magnitude and linear a geometry, whereas the silicon compound has a trans-bending angle of $137.44(4)^{\circ}$. The bending is explained through the mixing of an in-plane $\pi$ orbital (HOMO) with a low lying $\sigma^{*}$ orbital (LUMO).

The first conjugated systems with $\mathrm{Si}=\mathrm{Si}$ double bonds were pioneered in 1997 by Weidenbruch et al. ${ }^{[90]}$ They were obtained by lithiation of a symmetric disilene and subsequent addition of mesitylbromide. The resulting bromine disilene then reacts with the remaining lithiated disilene to the hexaaryltetrasilabuta-1,3-diene (Scheme 5).
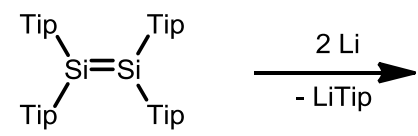

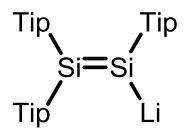

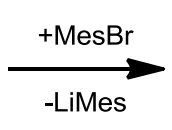

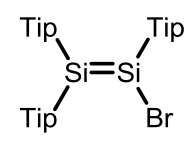

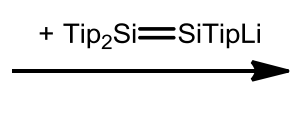

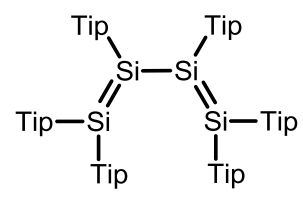

Scheme 5 synthesis of hexaaryltetrasilabuta-1,3-dien. 
After the $\mathrm{Si}=\mathrm{Si}$ double bonding has attracted a great deal of interest and $\mathrm{Si}=\mathrm{Si}$ double bonds were routinely isolated at room temperature, the focus turned on the possible existence of aromatic silicon compounds. Märkl et al. described the synthesis of 2,6-bis(trimethylsilyl)-1,4-di-tert-butylsilabenzene in 1988 stabilized only at $-100^{\circ} \mathrm{C}$ by the coordination of a Lewis-base solvent. The synthesis could only be proven from its relatively high field ${ }^{29} \mathrm{Si}$ NMR chemical shift ( $\delta=26.8 \mathrm{ppm}$ ). The successful synthesis of a stable silicon-substituted analogue of benzene was not accomplished until the year 2000 by Tokitoh et al. ${ }^{[91]}$ The synthesis started with stannacyclohexadiene over a ring opening reaction with Tbt-substituted trihydrosilane (Tbt = 2,4,6-tris[bis(trimethylsilyl)methyl]phenyl). After ring closure and chlorination the final silabenzene could be obtained via salt elimination (Scheme 6).

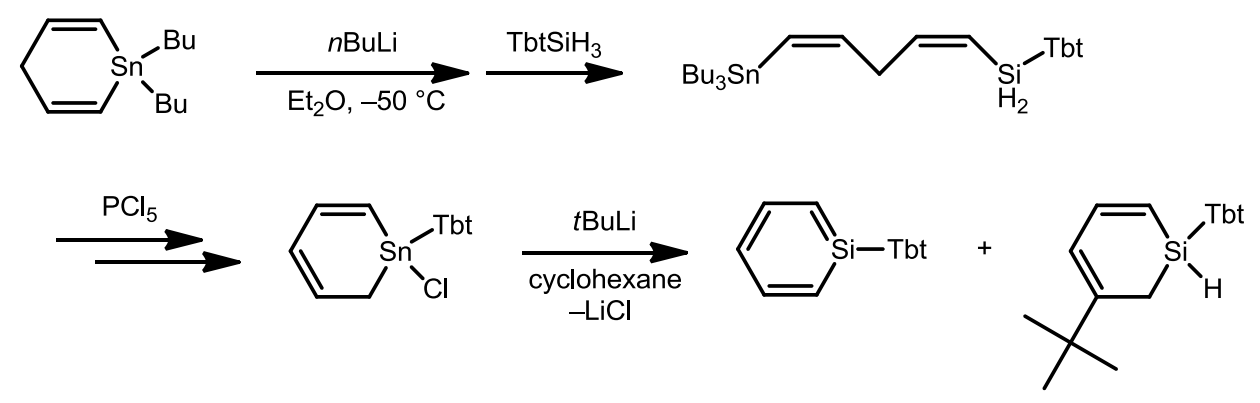

Scheme 6 Synthesis of silabenzene by Tokitoh et al. ${ }^{[91]}$

At the same time, Ando et al. independently reported the synthesis of 1,4-disila-(Dewar)-benzene by thermolysis of a stable bis-(silirene) in a degassed sealed tube at $100{ }^{\circ} \mathrm{C}$ in $\mathrm{C}_{6} \mathrm{D}_{6}$ (Scheme 7). ${ }^{[92-93]}$
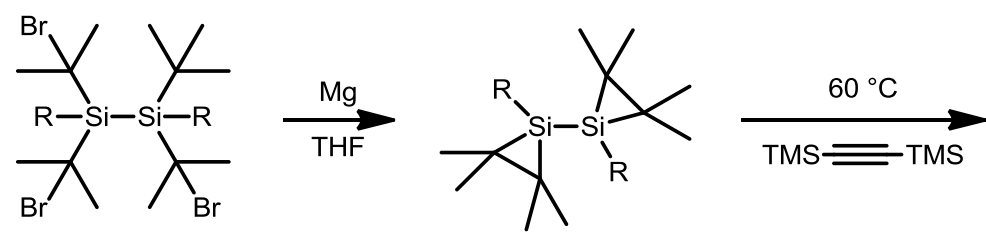

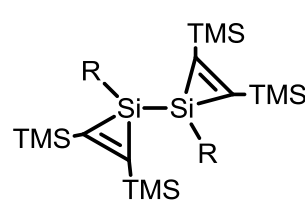

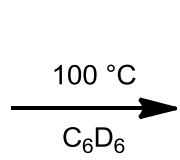

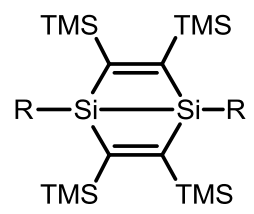

$\mathrm{R}=\mathrm{Me}, \mathrm{Et}, \mathrm{Ph}$

Scheme 7 Synthesis of 1,4-disila(Dewar-benzene).

Seven years later Sekiguchi et al. accomplished the synthesis of 1,2-disilabenzene by reacting $\mathrm{RSi} \equiv \mathrm{SiR}\left(\mathrm{R}=\mathrm{Si}\left(\mathrm{CH}\left(\mathrm{SiMe}_{3}\right)_{2} i \mathrm{Pr}\right)\right.$ with $\mathrm{Ph}-\mathrm{C} \equiv \mathrm{C}-\mathrm{H}$ in a formal $[2+2+2]$-cycloaddition reaction. ${ }^{[94]}$

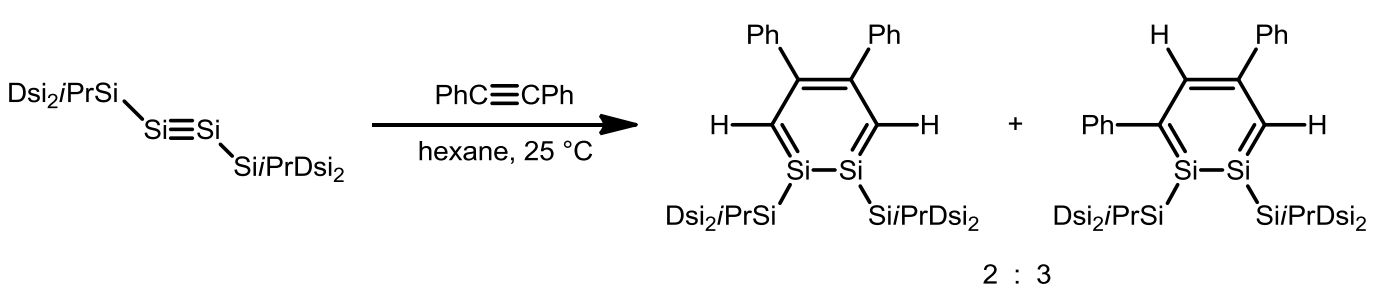

Scheme 8 Synthesis of 1,2-disilabenzene. Dsi $=\mathrm{CH}\left(\mathrm{SiMe}_{3}\right)_{2}$ 
Recently, in a cooperative effort of Stalke and the Roesky group the synthesis of a 1,4-disilabenzene by reaction of $\left[\left\{\mathrm{PhC}(\mathrm{NtBu})_{2}\right\} \mathrm{Si}\right]_{2}$ with diphenyl alkine was reported. ${ }^{\left[{ }^{[93]}\right.}$ With this ring structure, the question arose whether it is a 1,4-disilabenzene derivative or a diradical. The ${ }^{29} \mathrm{Si}$ NMR resonance was observed at $-18.05 \mathrm{ppm}$. The ring system is nearly planar and contains $6 \pi$-electrons. ESR measurements did not support the existence of a diradical. The bond lengths of the $\mathrm{Si}-\mathrm{C}$ and $\mathrm{C}-\mathrm{C}$ bonds in the ring show a shortening compared to the respecting single bonds from literature and are longer than their respective double bonded congeners. Full DFT gas phase optimizations carried out for the singlet and for the triplet state found the singlet state preferred by $10.61 \mathrm{kcal} \cdot \mathrm{mol}^{-1}$ with a high HOMO-LUMO gap of $34.67 \mathrm{kcal} \cdot \mathrm{mol}^{-1}$. The NICS(1) value of -3.64 indicate slightly aromatic character. The HOMO with a nodal plane in the ring plane resembles the equivalent orbitals from benzene in shape and symmetry but the degeneracy of the HOMO is lost.<smiles></smiles>

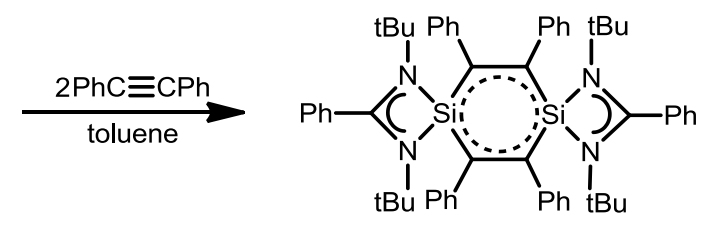

Scheme 9 Synthesis of 1,4-disilabenzene.

Furthermore, many kinds of derivatives of tetrasilacyclobutadiene were synthesized. ${ }^{[95-98]}$ They became feasible through the work of Sekiguchi et al. about the synthesis of cyclobutadiene dianions of tetreles. ${ }^{[99,96,100]}$ Tetrasilacyclobutadienes are impressive ligands in $\eta^{4}$-metal complexes, as the $\eta^{4}$-tetrasilacyclobutadiene (Scheme 10) from Sekiguchi et al. turned out to have a strong $\pi$ donating ability of the silacyclobutadiene moiety compared to that of its carbon counterpart. ${ }^{[97]}$<smiles>[R][Si]1(Br)[Si]([R])(Br)[Sn]([R])(Br)[Si]1(Br)Br</smiles>

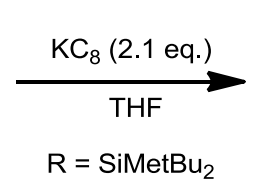<smiles>[R][SiH]1[Si]([R])(Br)[Si]1([R])Br</smiles>

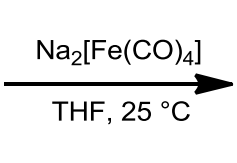

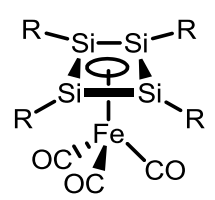

Scheme 10 Tetrasilacyclobutadiene in transition metal complexes.

Suzuki et al. described an antiaromatic homologue of cyclobutadiene. ${ }^{[101-102]}$ According to Hückels rule, molecules containing rings with $(4 n+2)$ delocalized $\pi$-electrons are most stable. In fact, cyclobutadiene is a very unstable molecule, but it gains extra stabilization from a covalent Jahn-Teller distortion. In cyclobutadiene the electrons are unpaired in the highest occupied level and placed into two degenerate singly occupied molecular orbitals (SOMOs). This leads to a rectangular molecule where two nonbonding electrons in the SOMOs are paired and occupy the HOMO, which is lower in energy. ${ }^{[103]}$

The synthesis of a Si analogue of cyclobutadiene by Suzuki et al. (Scheme 11, A) is remarkable, because $\mathrm{Si}=\mathrm{Si}$ bonds are much less stable than $\mathrm{C}=\mathrm{C}$ bonds and the $\mathrm{Si}_{4}$ ring of $\mathbf{A}$ would be further destabilized by antiaromaticity. ${ }^{[101-102]}$ The large 1,1,7,7-tetraethyl-3,3,5,5-tetramethyl-s-hydrindacen-4-yl (EMind) substituents kinetically stabilize the molecule and prevent dimerization. In comparison to $\mathrm{C}_{4} \mathrm{H}_{4}$, A has an unexpected rhombic structure. The four $\mathrm{Si}-\mathrm{Si}$ bond lengths differ by less than $0.03 \AA$ and are intermediate between typical Si-Si single $(2.34 \AA)$ and double $(2.14 \AA)$ 
bonds. Suzuki et al. explain this structural property by the stabilization of A from a polar Jahn-Teller distortion. This leads to the charge separated structure in Scheme 11.

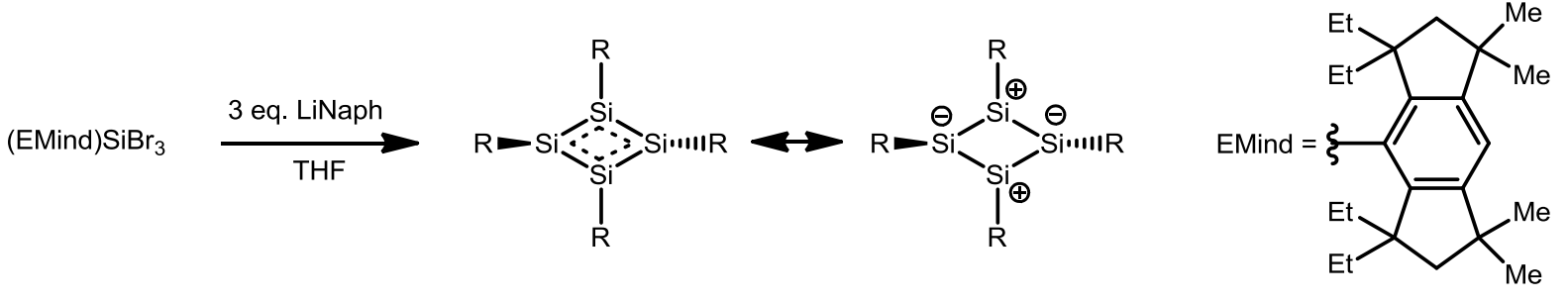

A

Scheme 11 Synthesis and two resonance structures of $\mathbf{A}$. $R=$ EMind.

The chemistry of the polyhedranes was enriched by Sekiguchi et al. with the synthesis of hexasilaprismane. For the synthesis of strained polyhedranes, the proper choice of both starting compounds with a suitable substituent and metals as reducing agent is of crucial importance. The metal should not be able to cleave the formed Si-Si bond. The hexasilaprismane of Sekiguchi et al. (Scheme 12) turned out to be fairly stable in the solid state against air for months. All the Si-Si bonds are slightly elongated with an average bond length of $2.38 \AA$ in comparison with normal $\mathrm{Si}-\mathrm{Si}$ single bonds of $2.34 \AA$. The 2,6-diisopropylphenyl groups are arranged in a screw-shaped manner so that they effectively cover the skeleton of the prismane.

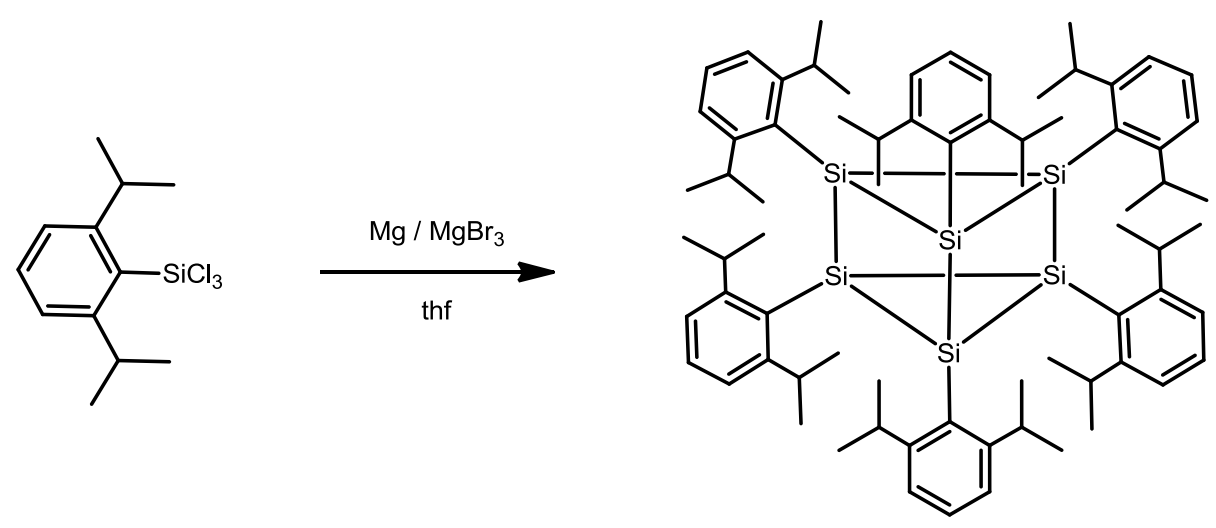

Scheme 12 Synthesis of hexasilaprismane.

The first homonuclear cluster with naked silicon(0) was synthesized by Wiberg. ${ }^{[104]}$ Their original intention was to synthesize the a dimer of tetrasilatetrahedrane ${ }^{[105]}$ by a metathesis of $\mathrm{MSi}_{4} \mathrm{R}_{3}{ }_{3}$ $\left(\mathrm{R}^{*}=\mathrm{SitBu}_{3}\right.$, "supersilyl") and $\mathrm{R}_{3}^{*} \mathrm{Si}_{4} \mathrm{X}(\mathrm{M}=$ alkali metal, $\mathrm{X}=$ Halogen). The metallation did not work as expected (Scheme 13). The reaction of the tetrasilatetrahedrane with iodine halogenated the tetrahedrane twice to the already known diiodotetrasupersilylcyclotetrasilene. ${ }^{[95]}$ A subsequent excess of iodine and treatment with $\mathrm{NaR}^{*}$ resulted in the unexpected cluster arrangement (Scheme 13). 


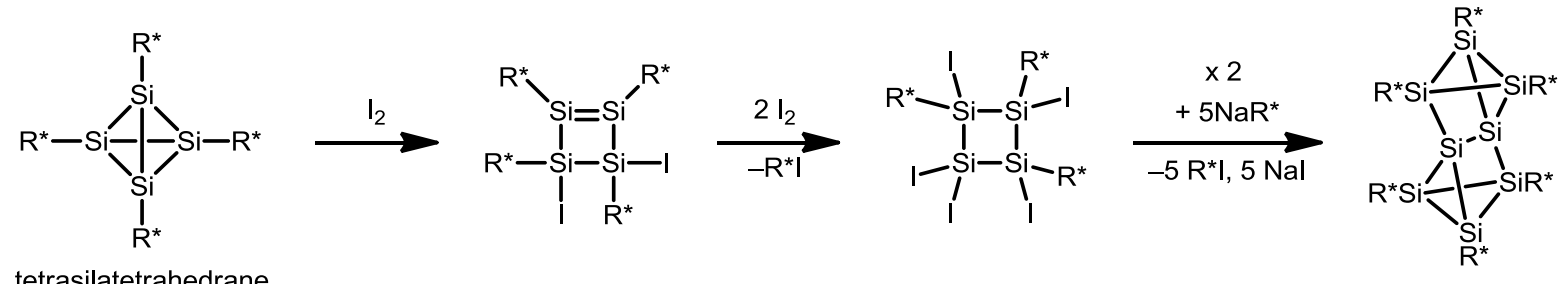

tetrasilatetrahedrane

Scheme 13 Synthesis of $\mathrm{Si}_{8}\left(\mathrm{SitBu}_{3}\right)_{6}, \mathrm{R}^{*}=\mathrm{SitBu}_{3}$.

With regards to homonuclear systems the Scheschkewitz group made groundbreaking progress with the isolation of ring ${ }^{[106-107]}$ and cage ${ }^{[108]}$ isomers (1 and 2) of hexasilabenzene (Scheme 14). Until then stable aromatic homonuclear silicon compounds were restricted to five or less ring atoms. ${ }^{[109,99,110,97,111-113]}$

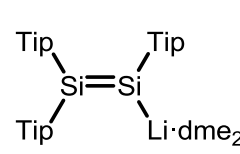

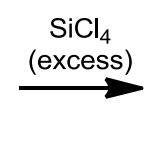<smiles></smiles>
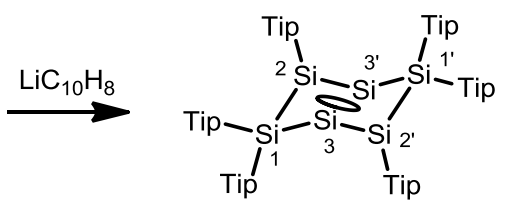

Scheme 14 Synthesis of the tricyclic isomer of hexasilabenzene (1). (Tip = 2,4,6-triisopropylphenyl)

The isomer 1 (Scheme 14) forms dark-green coloured crystals and crystallizes in the monoclinic space group $P 2_{1} / n$ with the midpoint of the central silicon ring residing on an inversion center. The asymmetric unit contains half a molecule and one benzene molecule as lattice solvent (structures with different lattice solvents are also published) ${ }^{[106,108]}$. The central motif of 1 consists of a tricyclic arrangement of silicon atoms in a chair conformation similar to the fully saturated compound previously reported by Kira et al. ${ }^{[14]}$ Two out of six silicon atoms are substituted by two Tip ligands, two by only one and two by none, only bound to three neighboring silicon atoms (Scheme 14). 1 exhibits a rhombic $\mathrm{Si}_{4}$-ring structure in the center, and two opposing $\mathrm{SiTip}_{2}$ units pointing up- and downwards with respect to the $\mathrm{Si}_{4}$ plane. The rhombohedral distortion is apparent in the diagonal Si3-Si3' distance of $2.70638(16)$ A which is about 17 \% longer than typical Si-Si single bonds.<smiles>[R][Si]1([R])[Si]([R])([R])[Si]([R])([Si]2([R])[Si]([R])([R])[Si]2([R])[R])[Si]1([R])[R]</smiles>

Scheme 15 Resonance formula of 1 . ( $R$ = Tip, 2,4,6-triisopropylphenyl)

Scheschkewitz et al. explained in the context of their analytical data, that the "mobile" electrons of 1 would be two $\pi$-, two $\sigma$ - and two nonbonding electrons cyclically delocalized over the four central silicon atoms. They use the term "dismutational aromaticity" for this type of cyclic delocalization. It involves a formal interruption of the $\sigma$-framework by two saturated $\mathrm{SiTip}_{2}$ homobridges. ${ }^{[115]}$ Because of the topological similarities to singlet diradicals of the Niecke type ${ }^{[116]}$ they did several calculations to elucidate the molecule properties. The optimization of the ${ }^{3} A_{u}$ state revealed a much longer diagonal distance (3.069 $\AA$ ) between the unsubstituted silicon atoms than was observed experimentally. The adiabatic singlet-triplet gap of $E_{S-T}=24.1 \mathrm{kcal} \cdot \mathrm{mol}^{-1}$ and over $90 \%$ contribution of a single closed-shell configuration supports a low diradical character of 1.

Later Scheschkewitz et al. reported an isomer of $\mathbf{1}$ which is obtainable by thermal or photolytic rearrangement (Scheme 16). ${ }^{[108]}$ 
<smiles>[R][Si]1([R])[Si]([R])([R])[Si]([R])([R])[Si]1([R])[R]</smiles>

Scheme 16 Rearrangement from 1 to 2 . ( $R$ = Tip, 2,4,6-triisopropylphenyl)

The structure of $\mathbf{2}$ consists of a silapropellane where two of the "propeller blades" are bridged by an additional $\mathrm{SiTip}_{2}$ unit. It turned out to be thermodynamically very stable. Scheschkewitz et al. could show that the $\Delta E_{S-T}=50.5 \mathrm{kcal} \cdot \mathrm{mol}^{-1}$ for a calculated model of 2 with 2,6-i $\mathrm{PrC}_{6} \mathrm{H}_{3}$ was much higher than in 1. It can be distilled at $10^{-2} \mathrm{mbar}$ and about $350^{\circ} \mathrm{C}$. The kinetic stability of $\mathbf{2}$ is much lower than 1-it decomposes within minutes in air as solution or solid. The ${ }^{29} \mathrm{Si} N \mathrm{NR}$ spectrum of 2 in $\left[D_{6}\right]$ benzene showed a remarkable dispersion of shifts $(\Delta \delta=448.8 \mathrm{ppm})$. They could explain the shifts with calculations and cluster currents as well (Figure 9). The strongest downfield shift was observed for the bridging silicon atom Si4 with $174.6 \mathrm{ppm}$. Such a strong shift would normally be a $\mathrm{sp}^{2}$-hybridized silicon atom. They explained it with a similar cluster current as in $\mathbf{1}$ where Si3 and $\mathrm{Si}^{\prime}(-274 \mathrm{ppm})$ are in a diatropic current loop, which thus exerts a magnetic shielding by back induction. This strong current vortex excludes the close-by lying Si4 atom, which is in turn surrounded by a distinct paratropic current vortex, leading to the unusual low-field resonance. The main current vortex branches around $\mathrm{Si2}$, $\mathrm{Si2}^{\prime}$ so that the shielding effect cancel out. This leads to a relatively small chemical shift of $-7.5 \mathrm{ppm}$. An also small shift has Si1 with $14.8 \mathrm{ppm}$.

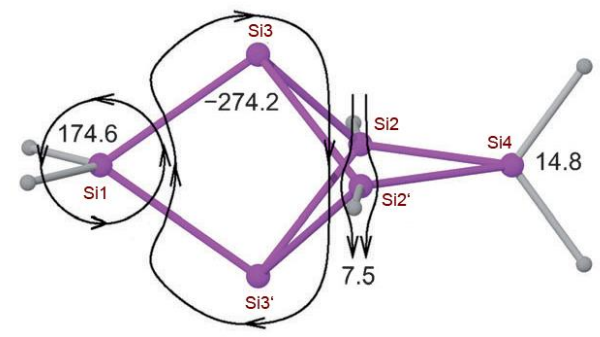

Figure 9 Most significant ring currents in $\mathbf{2}$ from Scheschkewitz et al.

The first unbridged [1.1.1]silapropellane was synthesized by Breher et al. (Scheme 17) ${ }^{[117-118]}$ but with only $1 \%$ yield. Before that, only two other compounds with "naked" silicon atoms were known. ${ }^{[104,119]}$ The silapropellane of Breher et al. showed a bridgehead distance of 2.6360(10) $\AA$ which is significantly shorter than the distance of 2.7076(8) Å reported by Scheschkewitz et al. for 2. Remarkably is that the distance found in this work with $2.6417(1) \AA$ is more similar to the distance reported by Breher. Even the Si-Si distance from the calculated ground state geometry is with $2.6553 \AA$ A more similar to the distance reported by Breher et al. This discrepancy might be caused of the low energy barrier of silicon clusters on the potential energy surface. ${ }^{[120]}$ 


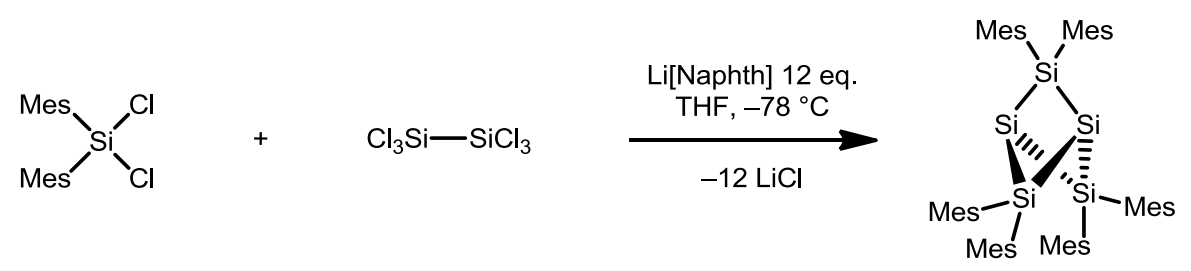

Scheme 17 Synthesis of a pentasila[1.1.1]propellane.

The excitation energy to the first triplet state $\left({ }^{3} A_{2}\right)$ was determined by UV-vis spectroscopy to be $52 \mathrm{kcal} \cdot \mathrm{mol}^{-1}$.

\subsubsection{Theoretical Predictions of Hexasilabenzenes}

Theoreticians tried to predict the stability and geometry of hexasilabenzene since the early 1980s. ${ }^{[121-127]}$ At that time the computer power and the level of theory were not able to predict its structure reliably. The introduction of electron correlation into the calculations changed the structure after geometry optimization from planar $D_{6 h}$ to the puckered chair like $D_{3 d}$ geometry (Scheme 18).<smiles>[SiH2]1[SiH2][SiH2][SiH2][SiH2][SiH2][SiH2]1</smiles>

$\mathrm{D}_{6 \mathrm{~h}}$<smiles>[SiH2]1[SiH2][SiH2][SiH2][SiH2]1</smiles>

$D_{3 d}$

Scheme 18 Schematic drawing of two possible geometries of $\mathrm{Si}_{6} \mathrm{H}_{6}$.

Due to the ambiguities of the electronic structure and properties, $\mathrm{Si}_{6} \mathrm{H}_{6}$ is still an area of research for theoreticians today. The fact that the planar geometry of $\mathrm{Si}_{6} \mathrm{H}_{6}$ is not a minimum energy isomer and has a vibrational instability that leads to a puckered structure is now common knowledge. ${ }^{[128-}$ ${ }^{132,120]}$ The distortion can be seen as a pseudo Jahn-Teller distortion by vibronic coupling between nondegenerate electronic states of proper symmetry. For distortion a strong coupling between unoccupied and occupied molecular orbitals has to take place. In solid state, the crystal packing also has a huge impact to the geometry since the energy differences for the distortion is in the range of the crystal packing forces. Also sterically demanding ligands and their variation should therefore have a big influence on the molecular geometry.
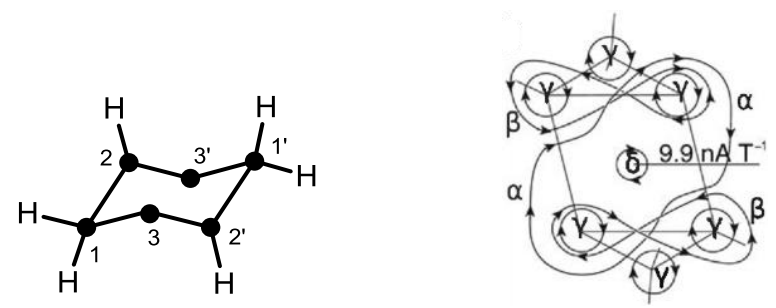

Figure 10 Schematic drawing of $1^{\prime}$ (left). Most significant ring currents from Scheschkewitz et al. (right).

Whether silicon analogues of benzene have aromatic character is still a point of constant debate. ${ }^{[125,133-135]}$ Therefore the isomer of hexasilabenzene $\left(\mathbf{1}^{\prime}\right)$ was analyzed in terms of NMR parameters to compare them with the aromatic behavior of benzene. ${ }^{[107]}$ The ${ }^{29} \mathrm{Si}$ NMR chemical shift of the silicon atoms with one substituent (Si2, Figure 10) was experimentally observed to be $\delta=$ 
$125 \mathrm{ppm}$ and in the region of tetrasilyl-substituted $\mathrm{Si}=\mathrm{Si}$ double bonds. A strong highfield shift was observed for Si3 and Si1 with $\delta=-89$ and $-85 \mathrm{ppm}$, respectively. These were in contrast to the calculated NICS(0) value of $\delta=-24 \mathrm{ppm}$ at the center of symmetry (benzene $\delta \approx-10 \mathrm{ppm}$ ). To rationalize these results, a magnetically induced probability density current field $\left(\mathrm{J}^{\mathrm{B}}\right)$ topology of $\mathbf{1}^{\prime}$ was investigated. ${ }^{[107]}$ The molecule was calculated at MP2/TZVPP level of theory with the simplification of hydrogen atoms instead of Tip substituents. They found a dominating ring-current contribution $\alpha$ around the unsubstituted Si3 and Si3' (Figure 10 right). This diamagnetic current shield Si3 and Si3' strongly. A counter-clockwise $\beta$ loop around Si2 and Si3 shields Si3 additionally and deshields Si2. This explains the large difference in the ${ }^{29} \mathrm{Si}$ NMR chemical shifts of atoms $\mathrm{Si2}$, Si2' and $\mathrm{Si} 3, \mathrm{Si}^{\prime}$. In addition the $\alpha$ loop shields Si1, resulting in a highfield ${ }^{29}$ Si NMR shift.

The integration of $\mathrm{J}^{\mathrm{B}}$ over the half plane of the molecule (black line in Figure 10 right) yielded in almost no paramagnetic contribution to the overall magnetic induced ring current of $9.9 \mathrm{nAT}^{-1}$. This value is almost the same as in benzene. A big difference to benzene was found in the NICS(0) value. While benzene has a central paramagnetic vortex which results in a NICS value of $-10 \mathrm{ppm}$, the $\operatorname{NICS}(0)$ value of $1^{\prime}$ was found to be $-24 \mathrm{ppm}$. This was attributed to the absence of the central paramagnetic current and excluded the classification as $6 \mathrm{e}^{-}$Hückel aromatic.

\subsubsection{Reactivity of 1 and 2}

The six-membered ring system 1 rearranges upon heating or UV irradiation to the red silicon cage compound $\mathbf{2}$ with a bridged propellane structure. ${ }^{[108]}$ An analogous transformation for fully saturated silicon compounds under irradiative conditions has been described by Kira and coworkers. $^{[114]}$

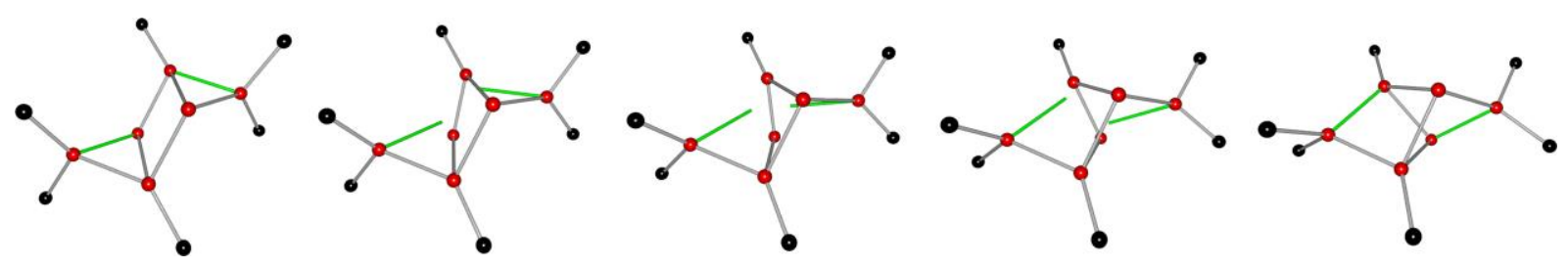

Figure 11 Proposed transition from the tricyclic isomer $\mathbf{1}$ (left) to the cage isomer $\mathbf{2}$ (right).

For the transition from the tricyclic isomer to the cage isomer, only a slight twist of the fourcyclic ring in $\mathbf{1}$ is necessary to form the cage compound. The transition of $\mathbf{1}$ to $\mathbf{2}$ via the reaction pathway in Figure 11 might be a possibility. The transformation proceeds via the breaking of the $\mathrm{Si1}-\mathrm{Si} 3$ and Si2-Si4 bonds in 1, accompanied with a twist of the four-membered silicon ring and subsequent formation of the new Si1-Si2 and Si3-Si4 bonds (green bonds in Figure 11).
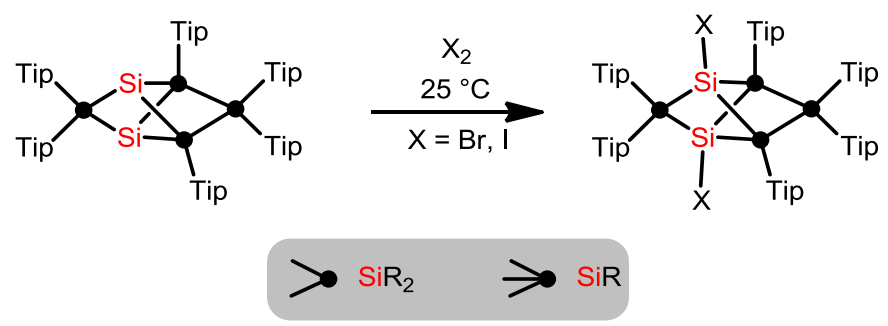

Scheme 19 Addition of halogens to 2. (Tip = 2,4,6-triisopropylphenyl) 
Compounds 1 and 2 react differently with halogens. ${ }^{[136]} \mathbf{2}$ reacts with halogens $\left(\mathrm{Br}_{2}, \mathrm{I}_{2}\right)$ at room temperature to a clean product (Scheme 19$)^{[108]}$ and the cyclic isomer $\mathbf{1}$ is much more reactive. The reaction with elementary halogens results in a crude mixture of products. The use of the mild halogenation reagent bismuth trichloride $\left(\mathrm{BiCl}_{3}\right)$ results in only four major products (Scheme 20 ).

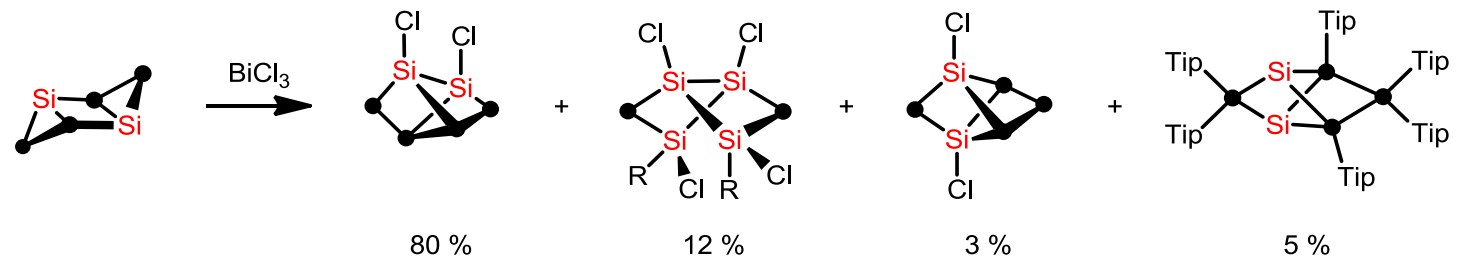

Scheme 20 Reaction of 1 with halogens.

\subsection{Refinement of Disordered Structures with XD2006}

The crystals for the measurement of $\mathbf{1}$ and $\mathbf{2}$ were synthesized and crystallized in the workgroup of Prof. Scheschkewitz (Lehrstuhl für Allgemeine und Anorganische Chemie der Universität des Saarlandes, 66125 Saarbrücken). The crystals from THF, benzene and hexane of both compounds form well shaped blocks with sharp edges and without inclusions and intergrowths. They can be handled in perfluorinated polyether oil without cooling under the microscope for a few minutes.

\subsubsection{Invariom Transfer to Disordered Structures}

The data sets of $\mathbf{1}$ and $\mathbf{2}$ were collected with a Bruker smart APEX II Ultra with a TXS Mo rotating anode, Incoatec mirror optics and an APEX II detector. The crystals were cooled with a Bruker Kryoflex II liquid nitrogen cooling device to $100 \mathrm{~K}$. For the multipole refinement of 1 and $\mathbf{2}$ a SHELXL starting model according to chapter 4.4.1 was used to generate an invariom model. ${ }^{[19]}$ The occupation of the disordered moieties was obtained from the SHELXL refinement and was also used for the XD2006 model. An initial XD2006 ${ }^{[137]}$ master and instruction file was created with $x$ dini according to the $X D 2006$ manual. ${ }^{[137]}$ The IAM refinement was then repeated with $x d l s m$ (the leastsquares refinement part of XD2006). The invariom model was essential to get a proper model for the disordered parts of the structure.

$X D 2006$ was never designed to model disorder, so the refinement of disordered structures with XD2006 needs some essential prerequisites:

- No disorder must be present in regions where properties have to be calculated. The free refinement of multipoles in disordered regions is possible but poorly supported by the current refinement programs. ${ }^{[138]}$ For example XD2006 only allows a limited number of constraints but to model disorder often more constraints as the program allows are needed. And the most important fact is that disorder makes the calculation of properties difficult because the required programs are not built for this purpose. The results are either not obtainable because of program crashes or not trustworthy.

- A proper starting model as in chapter 4.4.1 has to be made. For big molecules like $\mathbf{1}$ and $\mathbf{2}$ the atom names have to be clearly arranged and properly named. 
- The subsequent assignment of invarioms is easier if the atoms next to disordered atoms are also included in one of the disordered parts of the SHELXL refinement. Only by using this approach, InvariomTool can recognize the disorder.

- Negative part numbers have to be converted to positive numbers after SHELXL refinement, because the programs used in the subsequent refinement steps do not know the concept of negative parts. (SHELXL has the ability to set structure parts between PART -1 ... PART 0 to prevent bonds between the PART -1 and any other PART).

- The dataset should have as high resolution as in charge density refinement for a nondisordered structure.

- The Invariom database must have all entries for the atoms of the desired molecule. It is possible to generate invariom models with missing invarioms in the database but in this case the electro-neutrality of the molecule would not be fulfilled.

The program MolecoolQT $T^{[139]}$ is able to write a "xd_part.aux" file which defines the different parts of the disorder. When atoms are too close to each other the "xd_part.aux" needs some manual editing because MolecoolQT the treats them as one single atom.

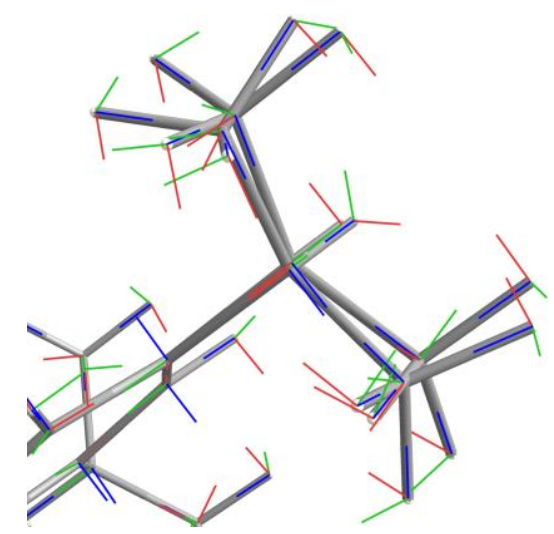

Figure 12 Local coordinate systems in the disordered isopropyl groups in $\mathbf{1}$ and $\mathbf{2}$ (red: $\mathbf{x}$, green: y, blue: $\mathbf{z}$ ).

A first run of InvariomTool generates an "Invariome.out" file. This file has to be inspected carefully whether all invarioms are assigned correctly. Badly assigned invarioms (mostly because InvariomTool cannot detect all cases of disorder) have to be edited by hand. The above procedure has to be performed in an iterative process until the file setup is correct. To model the disordered parts of a molecule, it is essential to have a correct "xd_part.aux" and "Invariome.in" file. MolecoolQT is important to visually control the input files and the orientation of every local coordinate system (Figure 12). The resulting XD master files have to be checked for completeness of atoms, constraints and dummy atoms before performing the multipole refinement.

\subsubsection{Procedure to Build the Invariom Database}

If structural segments of the molecule are missing in the invariom database, such invarioms must be generated. The generation of the invariom database is not very different to the transfer from the database to the molecule (chapter 4.4.2). Several steps to build the invariom database for the starting model of the subsequent refinement are required:

- The first step is to identify which invarioms are missing in the invariom database. For the missing invarioms, suitable model compounds have to be calculated. In the case of $\mathbf{1}$ and $\mathbf{2}$, the whole 
molecules were calculated, because the calculated density was also needed to compare it with the experimental results.

- Structure factors were calculated by analytical Fourier transformation of the real-space quantum chemical electron density with TONTO $^{[140-141]}$ using previously calculated wave functions (Gaussian ${ }^{[142]}$ at $\omega B$ 97XD/6-311G(d,p) level) ${ }^{[106,108]}$.

- A multipole refinement with XD2006 against the calculated structure factors was performed. The refinement was achieved as usual (chapter 4.4) but no positional parameters and ADPs were refined. Mono- and all multipoles for non-hydrogen atoms were refined as well as the $\kappa$ and $\kappa^{\prime}$ values for non-hydrogen atoms. To derive adequate parameters for the contraction of the hydrogen atoms, $\kappa$ and $\kappa^{\prime}$ values suggested by Volkov et al. were introduced and kept fixed during the refinement. ${ }^{[17]}$

- The refined multipole parameters have to be highly constrained in terms of symmetry in the molecule. For example, all methyl groups in $\mathbf{1}$ and $\mathbf{2}$ share the same multipole parameters. Otherwise the database would contain lots of different invarioms for the identical invariom notation.

- An invariom database with the multipole refinement results was created. To generate the database a special InvariomTool analyzes the invariom notation of each atom and writes the multipole parameters to the database. Such database entries are afterwards transferable to any molecule with the same molecular topology.

- The obtained invariom database was applied to the SHELXL starting model from which the multipole refinement against the experimental data was carried out.

\subsubsection{Multipole Refinement of 1}
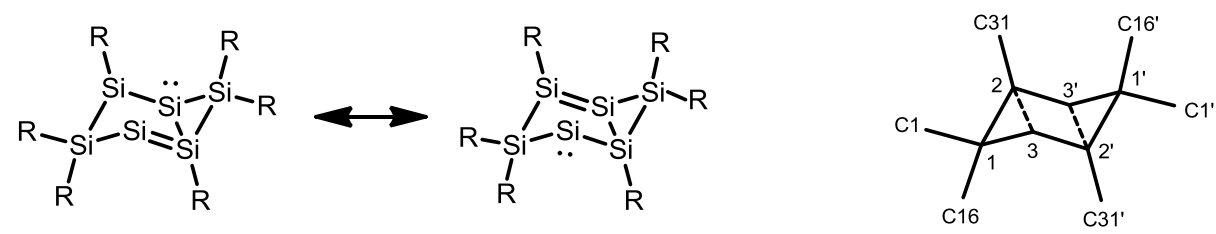

Scheme 21 Structure and numbering scheme of 1. ( $R$ = Tip, 2,4,6-triisopropylphenyl)

The multipole refinement using the atom-centered multipole model of Hansen and Coppens ${ }^{[16]}$ was carried out against $F^{2}$ with the full-matrix-least-squares refinement program $x d l s m$ implemented in the $X D 2006^{[137]}$ program package. The core and the spherical valence densities were composed of STO-Dirac-Fock atomic relativistic wavefunctions reported by Su, Coppens, Macchi (SCM bank file). ${ }^{[143-144]}$ The radial fit of these functions was described by the expansioncontraction parameters $\kappa$ and $\kappa^{\prime}$. The expansions over the spherical harmonics were truncated at the hexadecapolar level for all hetero atoms $\left(n_{l}=1\right.$ to 4$)$ and all multipoles of each atom shared the same $\kappa^{\prime}$-set (keep kappa constraint). The deformation densities of the hydrogen atoms were represented by hybrid scattering factors via a monopole, a bond directed dipole and a bond directed quadrupole. These quadrupoles were not refined, but fixed at the invariom level. The contraction of all atoms was described by $\kappa$ and $\kappa^{\prime}$ values implemented in the invariom database (chapter 5.2.2) and were kept fixed during the refinement (Kappa restricted refinement). 
Moreover, a riding model and distance constraints were applied for the hydrogen atoms during the first steps of the multipole refinement. In the final steps of the refinement the hydrogen atoms were refined freely. After each refinement step the distances of the hydrogen atoms were reset to atomic distances obtained in neutron diffraction experiments. ${ }^{[72,13]}$ The successive refinements led to a suitable model were convergence was reached.

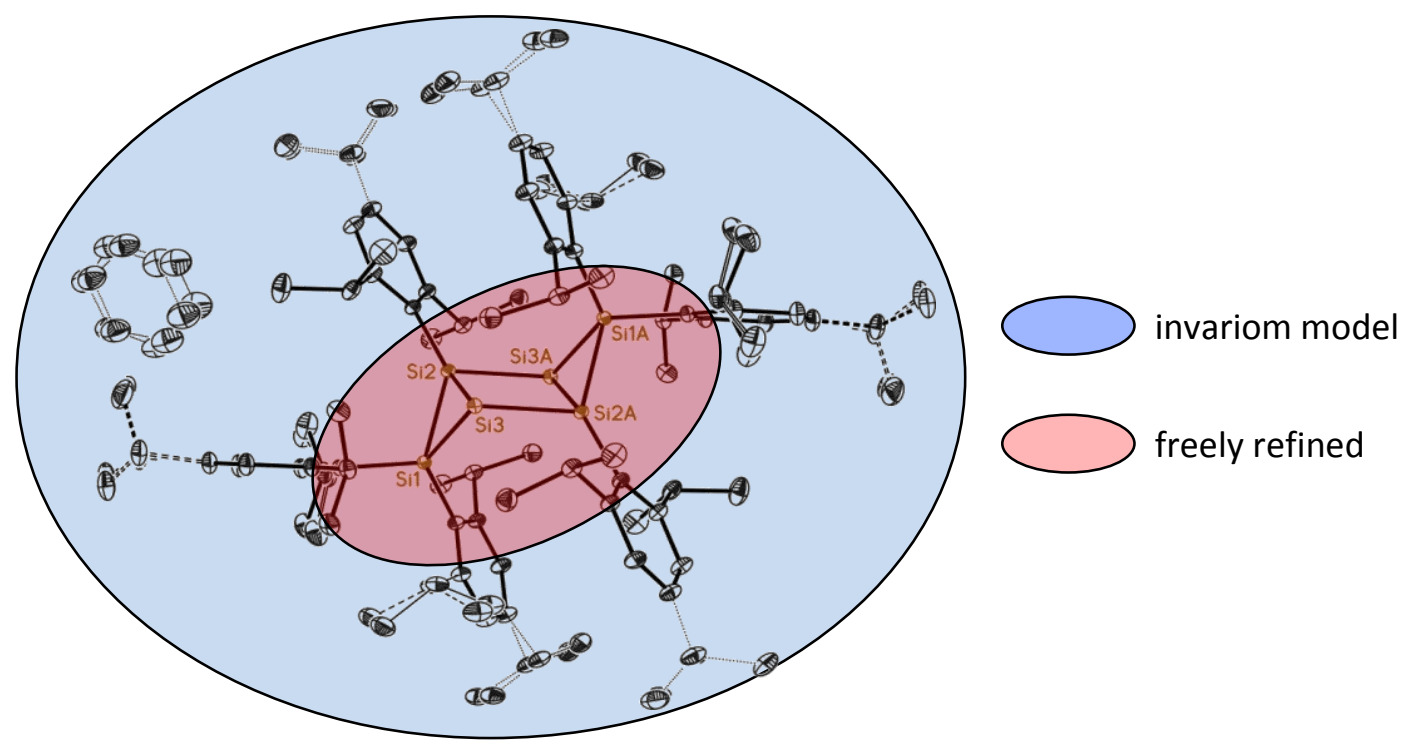

Figure 13 Schematic drawing of the invariom model used during the multipole refinements. Dotted lines are disordered regions.

Due to the disorder of the benzene rings in $\mathbf{1}$, as well as the Tip ligands, the multipole parameters of these parts were kept fixed at their invariom level. Normally restraints are used in cases of disorder to ensure reasonable bond lengths and vibrational parameters but XD2006 has no implementation of restraints. ${ }^{[12]}$ Therefore, the positional vibrational parameters of the solvent molecules and of the disordered Tip groups were not refined during the multipole refinements.

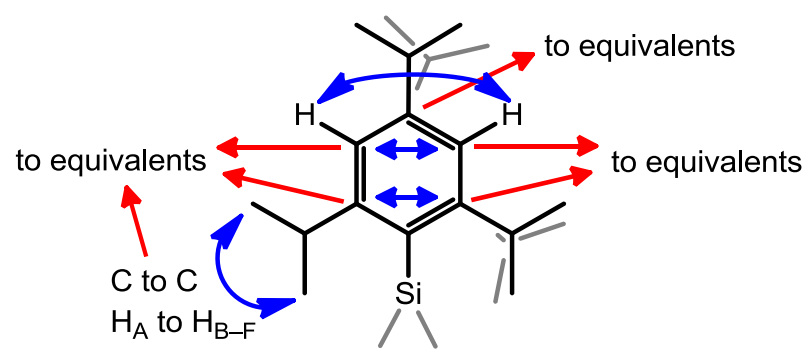

Scheme 22 Graphical representation of the chemical constraints during the refinement of 1. red: constraints to equivalent atoms in the other Tip groups. blue: internal constraints. grey: second part of disordered isopropyl groups.

Several models have been refined and compared, differing in the degree of applied chemical constraints and degree of refinement. If too many parameters on the Tip ligands were refined, the model got overfitted and was not consistent any more. The best results were obtained with the multipoles of the Tip ligands kept at invariom level, with the exception that the monopoles of the ring-carbon atoms were refined to allow charge transfer inside the molecule. Only the multipoles of the silicon atoms as well as their adjacent carbon atoms were refined completely free. A maximum amount of chemical constraints (Scheme 22) and symmetry restrictions for the multipolar functions led to a stable refinement. On the other hand the model had to be flexible enough to account for 
small differences of chemically equivalent atoms. The silicon atoms and their adjacent atom were refined without any chemical constraints. The density parameters were introduced in the refinement routines in a stepwise manner until convergence was reached (see chapter 4.4.3). Chemically equivalent or similar atoms were constrained to share the same expansion/contraction parameters, monopole and multipole populations in the beginning of the refinement. Hydrogen atoms with similar chemical environment were modeled with one set of parameters each.

The constraints of Scheme 22 were important to stabilize the refinement. Within each Tip ligand the methyl hydrogen atoms of the isopropyl groups were chemically constrained on each other. The use of invarioms led to a high data-to-parameter ratio (94 for 1), because many similar atoms were constraint to each other. Without the use of invariom scattering factors the refinement would not be possible with $X D 2006$.

Table 2 Crystallographic data after multipole refinement of $\mathbf{1 .}$

\begin{tabular}{|c|c|c|c|}
\hline CCDC number & 915313 & $\mathrm{Z}$ & 2 \\
\hline Empirical formula & $\mathrm{C}_{102} \mathrm{H}_{150} \mathrm{Si}_{6}$ & Crystal size $\left[\mathrm{mm}^{3}\right]$ & $0.22 \times 0.21 \times 0.14$ \\
\hline Formula weight $\left[\mathrm{gmol}^{-1}\right]$ & 1544.76 & $\rho_{\text {calc }}\left[\mathrm{Mgm}^{-3}\right]$ & 1.059 \\
\hline Temperature $[\mathrm{K}]$ & $100(2)$ & $\mu\left[\mathrm{mm}^{-1}\right]$ & 0.129 \\
\hline 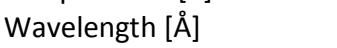 & 0.71073 & $F(000)$ & 1692 \\
\hline Crystal system & monoclinic & $\Theta$ range $\left[{ }^{\circ}\right]$ & 1.54 to 52.24 \\
\hline Space group & $P 2_{1} / n$ & Reflections collected & 731646 \\
\hline Unit cell dimensions $[\AA ̊]$ & & Unique reflections & 55651 \\
\hline$a=$ & $12.644(2)$ & $\mathrm{R}_{\text {int }} / \mathrm{R}_{\sigma}$ & $0.0490 / 0.0182$ \\
\hline$b=$ & $26.368(5)$ & Completeness to $\theta_{\max }$ & 99.7 \\
\hline$c=$ & $15.206(3)$ & data / parameters & 42922 / 455 \\
\hline$\alpha=$ & $90^{\circ}$ & GooF & 2.226 \\
\hline$\beta=$ & $107.183(3)^{\circ}$ & $\mathrm{R} 1[\mathrm{I}>2 \sigma(\mathrm{I})]$ & 0.029 \\
\hline$\gamma=$ & $90^{\circ}$ & $w R 2[I>1.4 \sigma(I)]$ & 0.064 \\
\hline Volume $\left[\AA^{3}\right]$ & $4843.3(16)$ & diff. peak / hole $\left[\mathrm{e}^{-3}\right]$ & $0.441 /-0.392$ \\
\hline Resolution $[\AA ̊]$ & 0.449 & & \\
\hline
\end{tabular}

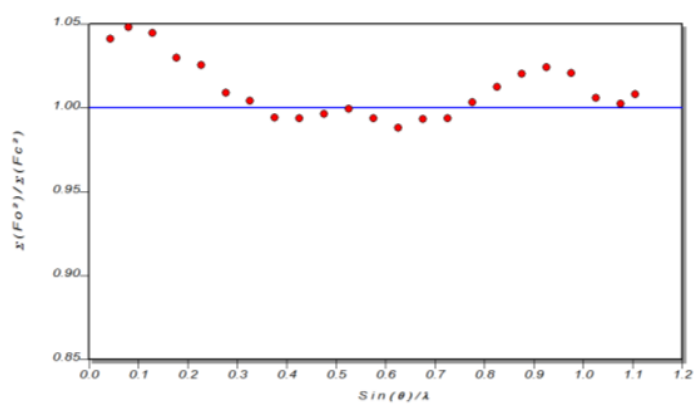

Figure 14 Variation of the scale factor against resolution after multipole refinement.

The variation of the scale factor in Figure 14 looks very well keeping in mind that a big amount of density was modeled with a rigid invariom model. The increase in the scale factor in the region lower resolution $\left(0-0.3 \AA^{-1}\right)$ probably come from the poorer fit of the disordered regions in the molecule. 


\subsubsection{Multipole Refinement of 2}<smiles>[R][Si]1([R])[Si]([R])([R])[Si]2([R])[Si]([R])([R])[Si]12[R]</smiles><smiles>ClC1(Cl)C2(Cl)C(Cl)(Cl)C3(Cl)C1(Cl)C23Cl</smiles>

Scheme 23 Structure and numbering scheme of 2. (R = Tip, 2,4,6-triisopropylphenyl)

The refinement of $\mathbf{2}$ with XD2006 was almost similar to the refinement of structure 1 . An invariom model from a previously calculated database (chapter 5.2.2) was transferred (chapter 4.4.2 and 5.2.1) into the starting model (chapter 4.4.1). The two disordered THF molecules were kept at invariom level were and not refined. The most prominent difference was that the isopropyl groups did not show as much disorder as in 1. Therefore, the model was allowed to be more flexible in the region of the Tip ligands. The most satisfactory model was the one where the monopoles of the Tip ligands ( $\mathrm{C}$ and $\mathrm{H}$ atoms) were refined freely with respect to strict chemical constraints (Scheme 24). In contrast to the model of $\mathbf{1}$, the parameters of the ligands were not constrained on each other (except the para-isopropyl groups. Earlier attempts to freely refine the multipoles in the Tip ligands did not lead to a consistent model. The multipole parameters of the silicon atoms and their adjacent carbon atoms were refined freely. All $\kappa$ and $\kappa^{\prime}$ parameters were kept fixed during all refinement steps (kappa restricted refinement). ${ }^{[145]}$

A known problem with second-row elements is that the equidistant normalized single Slater-type functions (see chapter 3.1) are inaccurate for heavy atoms with respect to accounting for electron density delocalized into the covalent bonds. There are many examples in the literature were different $n_{l}$ and $\zeta$ values were tested. ${ }^{[16,146-149]}$ The variation of $n_{l}$ and $\zeta$ values did not lead to a significant reduction of the residual density around the silicon atoms (chapter 5.4.8). Therefore, the $\zeta$ values were kept at the default values of the SCM database.

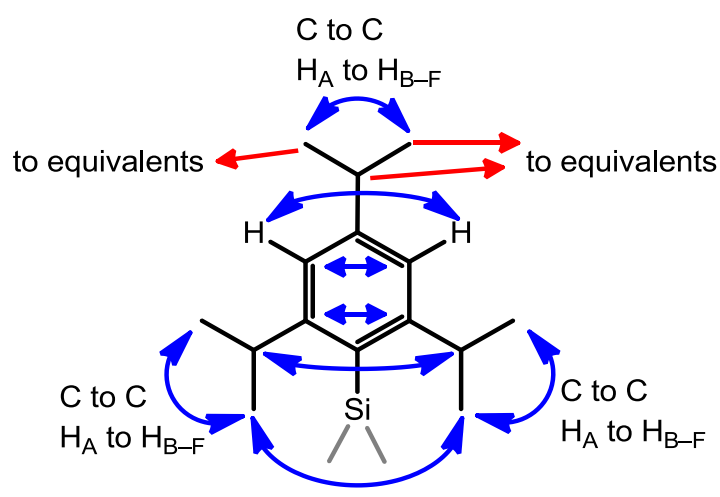

Scheme 24 Graphical representation of the chemical constraints during the refinement of 2 . Red: constraints to equivalent atoms in the other Tip groups. Blue: internal constraints.

It is remarkable how little residual density is left in the region of the Tip ligands (see chapter 5.3.7 and 5.4.8). This proves the fundamental suitability of the invariom model for the refinement of 1 and 2. 
Table 3 Crystallographic data after multipole refinement of $\mathbf{2}$.

\begin{tabular}{|c|c|c|c|}
\hline CCDC number & 915312 & Z & 2 \\
\hline Empirical formula & $\mathrm{C}_{102} \mathrm{H}_{162} \mathrm{O}_{3} \mathrm{Si}_{6}$ & Crystal size $\left[\mathrm{mm}^{3}\right]$ & $\begin{array}{l}0.191 \times 0.197 \times \\
0.272\end{array}$ \\
\hline Formula weight $\left[\mathrm{gmol}^{-1}\right]$ & 1604.86 & $\rho_{\text {calc }}\left[\mathrm{Mgm}^{-3}\right]$ & 1.102 \\
\hline Temperature $[\mathrm{K}]$ & $100(2)$ & $\mu\left[\mathrm{mm}^{-1}\right]$ & 0.134 \\
\hline Wavelength $[\AA \stackrel{\circ}{A}]$ & 0.71073 & $F(000)$ & 3528 \\
\hline Crystal system & monoclinic & $\Theta$ range $\left[{ }^{\circ}\right]$ & 1.51 to 55.81 \\
\hline Space group & $C 2 / c$ & Reflections collected & 618471 \\
\hline Unit cell dimensions $[\AA ̊]$ & & Unique reflections & 55022 \\
\hline$a=$ & $21.505(6)$ & $R_{\text {int }} / R_{\sigma}$ & $0.0337 / 0.0137$ \\
\hline$b=$ & $17.375(5)$ & Completeness to $\theta_{\max }$ & 98.1 \\
\hline$c=$ & $25.979(7)$ & data / parameters & 49372 / 683 \\
\hline$\alpha=$ & $90^{\circ}$ & GooF & 2.63 \\
\hline$\beta=$ & $94.554(10)^{\circ}$ & $\mathrm{R} 1[\mathrm{I}>2 \sigma(\mathrm{I})]$ & 0.022 \\
\hline$\gamma=$ & $90^{\circ}$ & $\mathrm{wR} 2[\mathrm{I}>1.4 \sigma(\mathrm{I})]$ & 0.056 \\
\hline Volume $\left[\AA^{3}\right]$ & $9676.3(50)$ & diff. peak / hole $\left[\mathrm{e} \AA^{-3}\right]$ & $0.411 /-0.494$ \\
\hline Resolution $[\AA ̊]$ & 0.430 & & \\
\hline
\end{tabular}
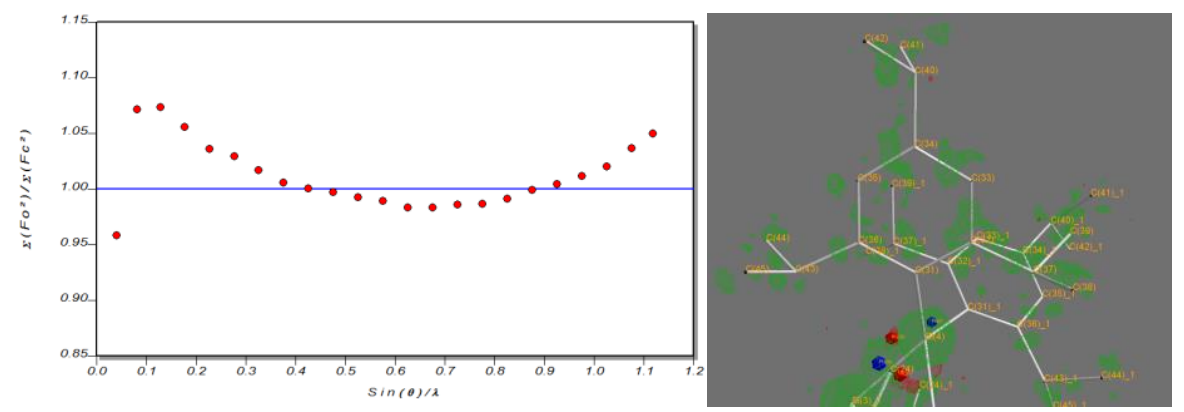

Figure 15 Variation of the scale factor against resolution (left) after refinement with one scale factor. Residual density at the Tip groups on Si 4 in 2 (right, at $0.11 \mathrm{e}^{-3}$ level).

The refinement of $\mathbf{2}$ showed significant variation of the scale factor distribution (Figure 15) (see chapter 4.2.3 for details on the scale factor). Since a proper scaling is important for correct modeling of the electron density (see Chapter 4.2.3), three different refinements with different resolution batches of scale factors were tested independently. The properties after the refinement with 1, 5 and 10 different scale factors were compared to determine the best refinement model. For this purpose a python script was developed which is able to divide a $h k l$ data set into $n$ equal sized batches based on resolution (in $\AA^{-1}$ ) limits (like "start, step, number of steps") or into selfdefined scale factor resolution batches (like "0.2, 0.5, 0.8, 1.0").

As quality indicators of the different refinement models, the scale factor variation over the resolution, the residual density in one moiety of the molecule, and the $R$-value of $\rho(\boldsymbol{r})$ as well as $\nabla^{2} \rho(\boldsymbol{r})$ at the bond critical point of 14 different bonds (all Si-Si and Si-C bonds) were compared. For the calculation of the $R$-value the results from the refinement against theory data (Chapter 5.2.2) was used as calculated observation.

$$
R(x)=\frac{\sum\left(\left|x_{o}\right|-\left|x_{c}\right|\right)}{\left|x_{o}\right|}
$$



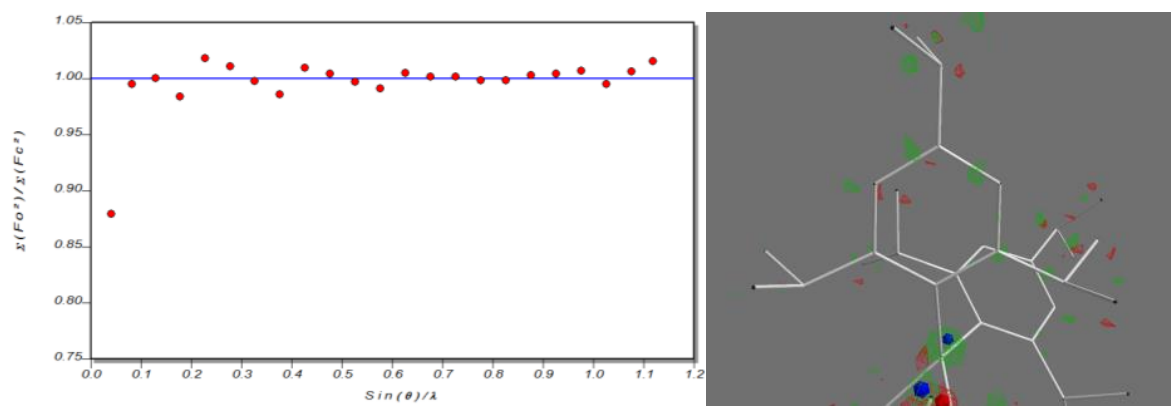

Figure 16 Variation of the scale factor against resolution (left) after refinement with five scale factors. Residual density at the Tip groups on $\mathrm{Si} 4$ in $\mathbf{2}$ (right, at $0.11 \mathrm{e}^{-3}$ level).
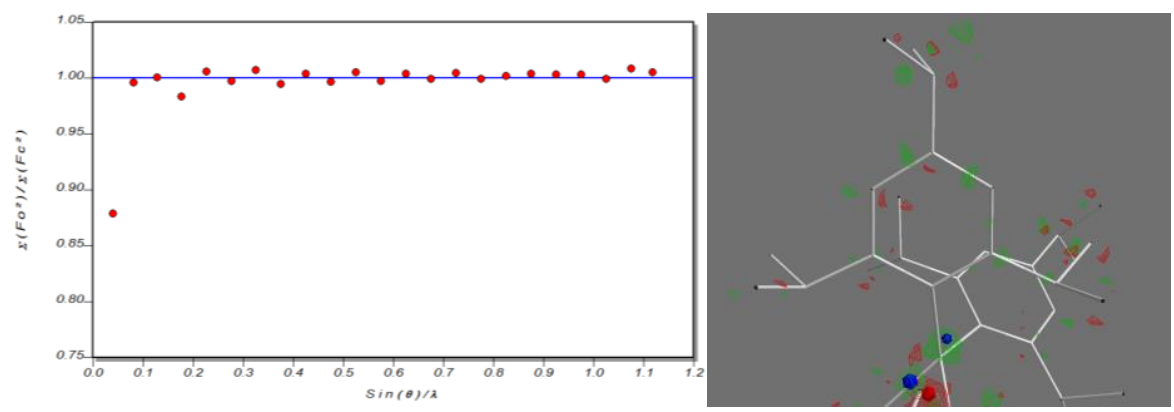

Figure 17 Variation of the scale factor against resolution (left) after refinement with ten scale factors. Residual density at the Tip groups on $\mathrm{Si} 4$ in 2 (right, at $0.11 \mathrm{e}^{-3}$ level).

Table 4 Quality indicators after multipole refinement of $\mathbf{2}$ with $n$ scale factors.

\begin{tabular}{ccc}
$\begin{array}{c}n \text { scale } \\
\text { factors }\end{array}$ & $R\left[\rho\left(\boldsymbol{r}_{B C P}\right)\right]$ & $R\left[\nabla^{2} \rho\left(\boldsymbol{r}_{B C P}\right)\right]$ \\
\hline \hline 1 & $2.2 \%$ & $19 \%$ \\
5 & $1.6 \%$ & $14 \%$ \\
10 & $1.3 \%$ & $16 \%$ \\
\hline
\end{tabular}

The introduction of more than one scale factor improved the distribution of the scale factor against the resolution significantly (Figure 16 and Figure 17) as well as the residual density distribution. The $R$-value for $\rho\left(\boldsymbol{r}_{B C P}\right)$ dropped by $0.6 \%$ and $0.9 \%$ for 5 and 10 scale factors, respectively. Interestingly, the $R$-Value for $\nabla^{2} \rho\left(\boldsymbol{r}_{B C P}\right)$ drops with five scale factors but increases with ten scale factors.

The refinement using five scale factors was chosen because it was in better agreement with theory. The refinement with ten scale factors did not improve the model enough to justify the use of five more parameters.

\subsection{Topological Analysis of the Hexasilabenzene Isomer 1}

The following electron density analysis is focused on the properties that can be derived from experimental data. The theoretical details such as NMR resonance and density functional calculations were previously analyzed by Scheschkewitz et al. ${ }^{[106-107]}$ Nevertheless, all property values were compared to the respective values derived from the multipole refinement against calculated structure factors obtained from Fourier transform with the program TONTO ${ }^{[140-141]}$ from a 
previously calculated single-point wave functions (Gaussian ${ }^{[142]}$ at the $\omega B 97 X D / 6-311 G(d, p)$ level ${ }^{[106,108]}$ All values which are compared to the values obtained from the refinements against calculated (with TONTO) structure factors are given in brackets. Since the outer sphere of $\mathbf{1}$ was modeled by invarioms, no information on the topology of the electron density for these part of the molecules can be extracted. Therefore, the following study is only focused on the $\mathrm{Si}-\mathrm{Si}$ and $\mathrm{Si}-\mathrm{C}$ bonds of the molecules.

\subsubsection{Bond Critical Points and Molecular Graph of 1}

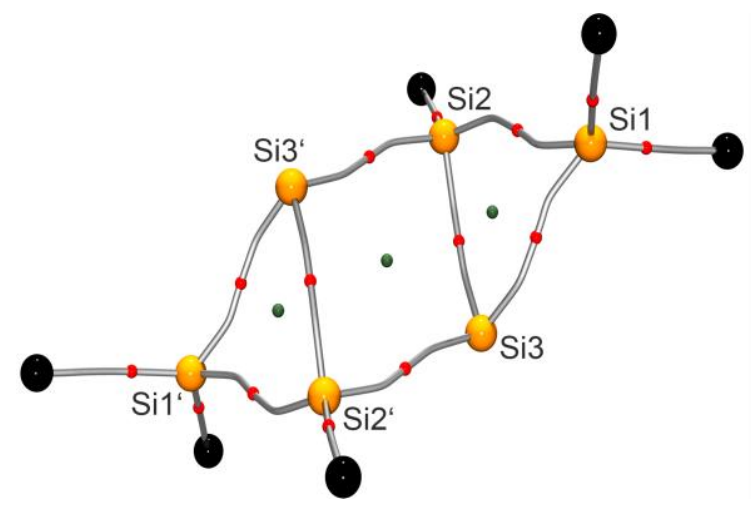

Figure 18 Molecular graphs of 1, depicting the curved bond paths, bond critical points (red) and ring critical points (green).

The triangle formed by Si1-Si2-Si3 (Figure 18) is a typical example of strained ring systems with bent bonds. ${ }^{[150,36,151-152]}$ The bond paths are extremely curved (Figure 18). The maxima in the deformation density are clearly outside of the straight line directly connecting the atoms (Figure 19). The deformation density map in Figure 19 shows directly that the bond between Si2 and Si3 contains a lot less density than the Si1-Si2,3 bonds. In Table 5 the density at the bond critical point for Si2-Si3 is just 0.466(7) [0.484] e $\AA^{-3}$ but 0.537(5) [0.524] e $\AA^{-3}$ and 0.545(6) [0.518] e $\AA^{-3}$ for Si1$\mathrm{Si} 2$ and $\mathrm{Si1}-\mathrm{Si} 3$, respectively.

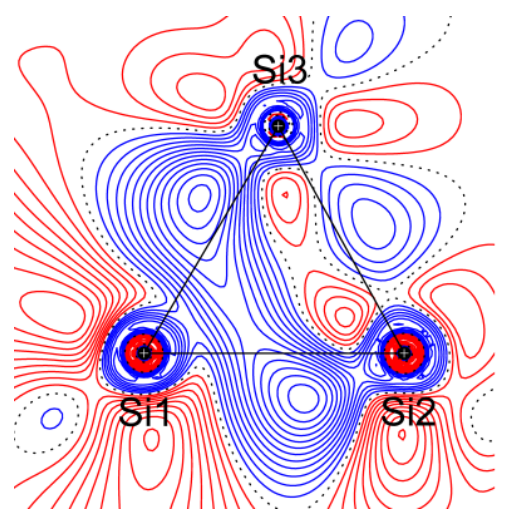

Figure 19 Static deformation density contour plot of 1 . Contour lines are drawn at $\pm 0.015,0.03, \ldots \mathrm{e} \AA^{-3}$ interval level; blue: positive; red: negative values.

Importantly, no BCP was found for a possible through-space interaction between $\mathrm{Si} 3$ and $\mathrm{Si3}$ '. This cannot be ruled out from the outset because (1) the structure might have biradicaloid character and a possibly more stable triplet state could be formed if an interstitial bond is present and (2) the distance between Si3 and Si3' is significantly longer ( $2.8 \AA$ ) than the other Si-Si bonds (2.4 Å). 

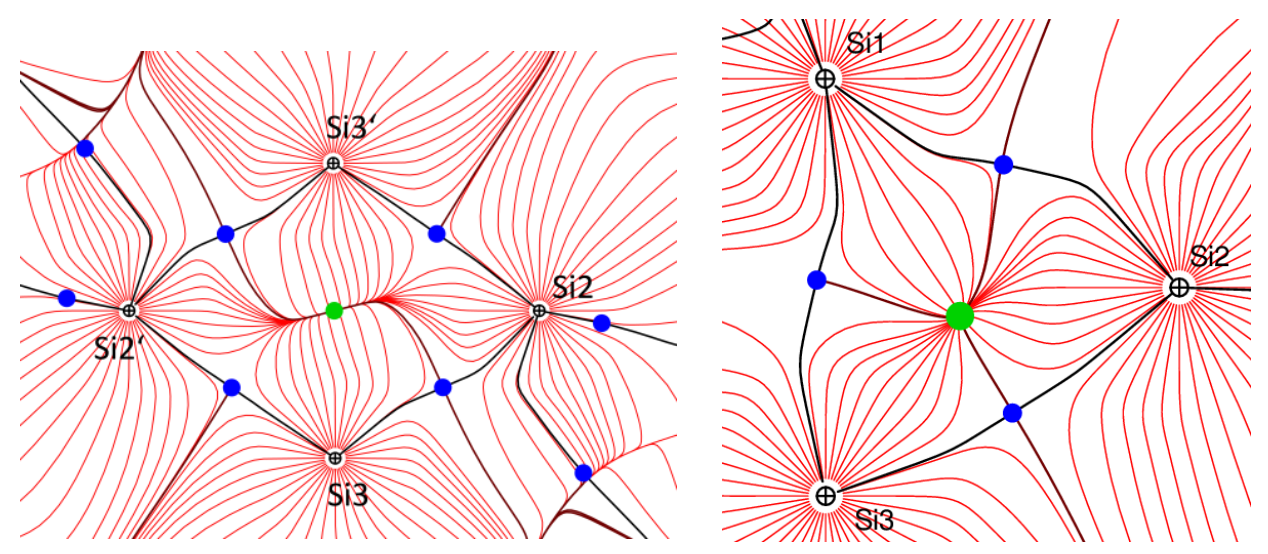

Figure 20 Gradient field of 1 (experimental data) in the plane of the four membered ring (left) and the three membered ring (right).

The gradient plot in Figure 20 shows the atomic basins in the four-membered ring plane. A ring critical point was found in the center. An interesting fact is that only one zero-flux surface is going through the center. Instead, the ring critical point resides on an s-shaped boundary which was unexpected. Presumably, the "missing" zero-flux surface is just close to be also existent. The adjacent three membered ring shows extremely curved bond paths between Si1 and Si2, Si3 with the ring critical point in the center.

Table 5 Topological parameters of the BCP and integrated atomic charges of 1 [theoretical values in brackets].

\begin{tabular}{|c|c|c|c|c|c|}
\hline bond $^{[a]}$ & $\rho(\mathbf{r})\left[\mathrm{e} \AA^{-3}\right]$ & $\nabla^{2} \rho(\mathbf{r})\left[\mathrm{e} \AA^{-5}\right]$ & $\epsilon_{\mathrm{BCP}}$ & $\eta_{B C P}$ & $\mathrm{Q}[\mathrm{e}]$ \\
\hline $\mathrm{SI} 1 *-\mathrm{SI} 2$ & $0.537(5) \quad[0.524]$ & $-1.645(8)[-1.798]$ & $0.20[0.33]$ & 1.32 [1.63] & $1.63[1.38]$ \\
\hline Si1-Si3* & $0.545(6) \quad[0.518]$ & $-1.628(8)[-1.616]$ & $0.21[0.24]$ & $1.28[1.34]$ & $-0.34[-0.14]$ \\
\hline $\mathrm{Si} 2 *-\mathrm{Si} 3$ & $0.466(7) \quad[0.484]$ & $-1.164(9)[-0.940]$ & $0.20[0.16]$ & $1.07[0.96]$ & $+0.62[+0.62]$ \\
\hline Si2'-Si3 & $0.595(11)[0.537]$ & $-2.285(12)[-1.798]$ & $0.10[0.33]$ & $1.72[1.56]$ & $-\quad[-]$ \\
\hline Si1-C1* & $0.826(7) \quad[0.736]$ & $+2.315(17)[+0.957]$ & $0.06[0.08]$ & $0.39[0.45]$ & $-0.83[-0.68]$ \\
\hline Si1-C16* & $0.853(7) \quad[0.736]$ & $+2.354(17)[+1.169]$ & $0.12[0.07]$ & $0.41[0.44]$ & $-0.83[-0.68]$ \\
\hline Si1-C31* & $0.776(8) \quad[0.756]$ & $+2.288(19)[+1.609]$ & $0.22[0.09]$ & $0.42[0.42]$ & $-0.76[-0.71]$ \\
\hline
\end{tabular}

Q: the charge of the $\left({ }^{*}\right)$-marked atom, derived by the difference of atomic number $Z$ and $\rho(\boldsymbol{r})$ integrated over the respective atomic basin.

\subsubsection{Integrated Charges and Valence Shell Charge Concentrations}

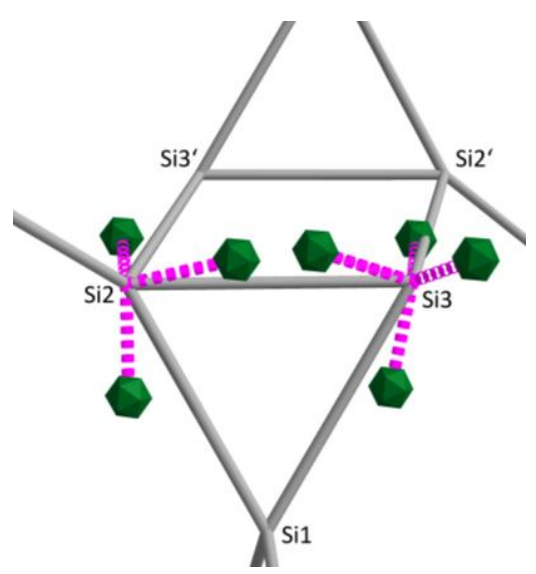

Figure 21 Arrangement of the VSCCs (green) found at Si2 and Si3. 
At the $\mathrm{Si} 3$ and $\mathrm{Si}^{\prime}$ atoms, a distinct valence shell charge concentration (VSCC) of -2.08 $[-1.39] \mathrm{eA}^{-5}$ is observed in the position where the lone pair in the Lewis diagram of the silicon(0) atom ( $\mathrm{Si} 3$ and Si3', Figure 21 and Figure 22) would be expected. The tetrahedral geometry of the VSCC arrangement in Si3 explains why there can be no interstitial bond path between $\mathrm{Si} 3$ and $\mathrm{Si}^{\prime}$. The VSCC from Si3 pointing to any other atom is only $37^{\circ}$ of from the Si2-Si3-Si2'-Si3' plane (Figure 21).

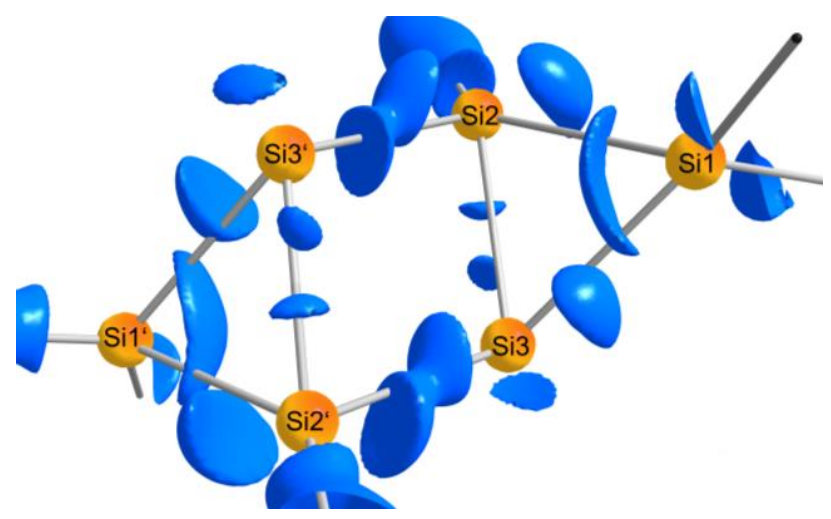

Figure 22 Laplacian distribution from experimental data around the silicon atoms of 1 at an isosurface level of $-1.9 \mathrm{eA}^{-5}$.

Formally one could assign the oxidation state $+\mathrm{Il}$ to $\mathrm{Si} 1,+\mathrm{I}$ to $\mathrm{Si} 2$ and zero to $\mathrm{Si} 3$ based on the Lewis diagram. The integration of the atomic basins according to Baders QTAIM gives a similar pattern of +1.6 e [ +1.4 e], +0.6 e $[+0.6$ e], -0.3 e [ -0.1 e], respectively. This documents a good agreement of experiment and theory. It is remarkable that the formally zero-charged silicon atoms are even negatively charged. Normally, a negative oxidation state of silicon is very unusual. The derived charges also show that the density is partly conjugated through the system. The different charges indicate that polarization in the silicon ring occurs: the higher electronegativities of the adjacent carbon atoms suggest a polarization especially of the Si-C bonds. 
Table 6 Integrated charges and VSCCs at selected atoms in $\mathbf{1}$ (Experimental data).

\begin{tabular}{|c|c|c|c|c|c|}
\hline & \begin{tabular}{|l} 
Integration \\
$\mathrm{Q}[\mathrm{e}]$
\end{tabular} & $\begin{array}{l}\text { integrated } \\
\text { Lagrangian }\end{array}$ & $\begin{array}{l}\text { VSCC } \\
\nabla^{2} \rho(\mathbf{r})\left[\mathrm{e}^{-5}\right]\end{array}$ & distance $[\AA]]$ & \begin{tabular}{|l} 
VSCC \\
direction
\end{tabular} \\
\hline Si(1) & 1.63 & $3.66 \cdot 10^{-4}$ & -2.54 & 0.906 & $\mathrm{Si} 2, \mathrm{Si} 3$ \\
\hline \multirow[t]{3}{*}{ Si(2) } & 0.62 & $1.13 \cdot 10^{-3}$ & -2.44 & 0.916 & $\mathrm{Si} 3$ \\
\hline & & & -4.10 & 0.903 & Si1 \\
\hline & & & -3.64 & 0.907 & $\mathrm{Si}^{\prime}$ \\
\hline \multirow[t]{4}{*}{ Si(3) } & -0.34 & $3.89 \cdot 10^{-4}$ & -2.08 & 0.912 & LP \\
\hline & & & -3.11 & 0.908 & Si2' \\
\hline & & & -3.87 & 0.892 & Si1 \\
\hline & & & -2.28 & 0.911 & $\mathrm{Si} 2$ \\
\hline \multirow[t]{3}{*}{$C(1)$} & -0.83 & $9.29 \cdot 10^{-4}$ & -30.57 & 0.482 & Si1 \\
\hline & & & -29.04 & 0.483 & C2 \\
\hline & & & -28.01 & 0.485 & C6 \\
\hline \multirow[t]{3}{*}{$C(16)$} & -0.83 & $1.27 \cdot 10^{-3}$ & -27.40 & 0.486 & C17 \\
\hline & & & -29.25 & 0.484 & $\mathrm{Si} 2$ \\
\hline & & & -30.07 & 0.481 & $\mathrm{C} 21$ \\
\hline \multirow[t]{3}{*}{$C(31)$} & -0.76 & $-4.55 \cdot 10^{-4}$ & -32.79 & 0.480 & C36 \\
\hline & & & -23.97 & 0.487 & Si2 \\
\hline & & & -27.42 & 0.485 & C32 \\
\hline
\end{tabular}

Table $\mathbf{7}$ Integrated charges and VSCCs at selected atoms in $\mathbf{1}$ (theoretical data).

\begin{tabular}{|c|c|c|c|c|c|}
\hline & \begin{tabular}{|l} 
Integration \\
$\mathrm{Q}[\mathrm{e}]$
\end{tabular} & $\begin{array}{l}\text { integrated } \\
\text { Lagrangian }\end{array}$ & $\begin{array}{l}\operatorname{VSCC} \\
\Delta^{2}(\rho)\left[\mathrm{e} \AA^{-5}\right]\end{array}$ & distance $[\AA ̊]$ & $\begin{array}{l}\text { VSCC } \\
\text { direction }\end{array}$ \\
\hline \multirow[t]{4}{*}{ Si(2) } & 1.38 & $-1.63 \cdot 10^{-3}$ & -3.68 & 0.922 & C32 \\
\hline & & & -3.33 & 0.913 & $\mathrm{Si} 1$ \\
\hline & & & -3.73 & 0.920 & $\mathrm{C} 4$ \\
\hline & & & -2.82 & 0.922 & $\mathrm{Si} 3$ \\
\hline \multirow[t]{4}{*}{ Si(3) } & 0.62 & $7.18 \cdot 10^{-4}$ & -3.21 & 0.915 & Si2 \\
\hline & & & -2.06 & 0.922 & $\mathrm{Si} 1$ \\
\hline & & & -2.68 & 0.923 & Si88 \\
\hline & & & -3.68 & 0.939 & $\mathrm{C} 60$ \\
\hline \multirow[t]{4}{*}{ Si(1) } & -0.14 & $6.84 \cdot 10^{-4}$ & -2.49 & 0.922 & Si90 \\
\hline & & & -2.63 & 0.916 & $\mathrm{Si} 2$ \\
\hline & & & -1.39 & 0.925 & LP \\
\hline & & & -2.20 & 0.913 & $\mathrm{Si3}$ \\
\hline \multirow[t]{3}{*}{$C(4)$} & -0.68 & $6.02 \cdot 10^{-4}$ & -23.72 & 0.487 & $\mathrm{Si} 2$ \\
\hline & & & -24.93 & 0.487 & $\mathrm{C} 5$ \\
\hline & & & -25.25 & 0.487 & $\mathrm{C} 10$ \\
\hline \multirow[t]{3}{*}{$C(60)$} & -0.71 & $-1.81 \cdot 10^{-4}$ & -23.63 & 0.488 & $\mathrm{Si3}$ \\
\hline & & & -25.77 & 0.486 & C61 \\
\hline & & & -25.74 & 0.486 & $\mathrm{C} 66$ \\
\hline \multirow[t]{3}{*}{$C(91)$} & -0.68 & $6.08 \cdot 10^{-4}$ & -25.25 & 0.487 & C92 \\
\hline & & & -23.72 & 0.487 & Si89 \\
\hline & & & -24.93 & 0.487 & $\mathrm{C} 97$ \\
\hline
\end{tabular}

The bonding between Si1 and Si2/3 is special. At Si1 only one VSCC directed to the bond path between Si2 and Si3 could be found from experimental data. This is a difference to the Laplacian distribution of the theoretical data where all four expected VSCCs could be found (Table 6). Instead of the expected bond-directed VSCCs for the Si1-Si2 and Si1-Si3 bond, one can see in the three dimensional distribution of $L(\boldsymbol{r})$ that there is a broad VSCC directed to the transannular bond (Figure 22). Also for Si2 the VSCC directed to the adjacent Tip ligand could not be found in the experimental data. 


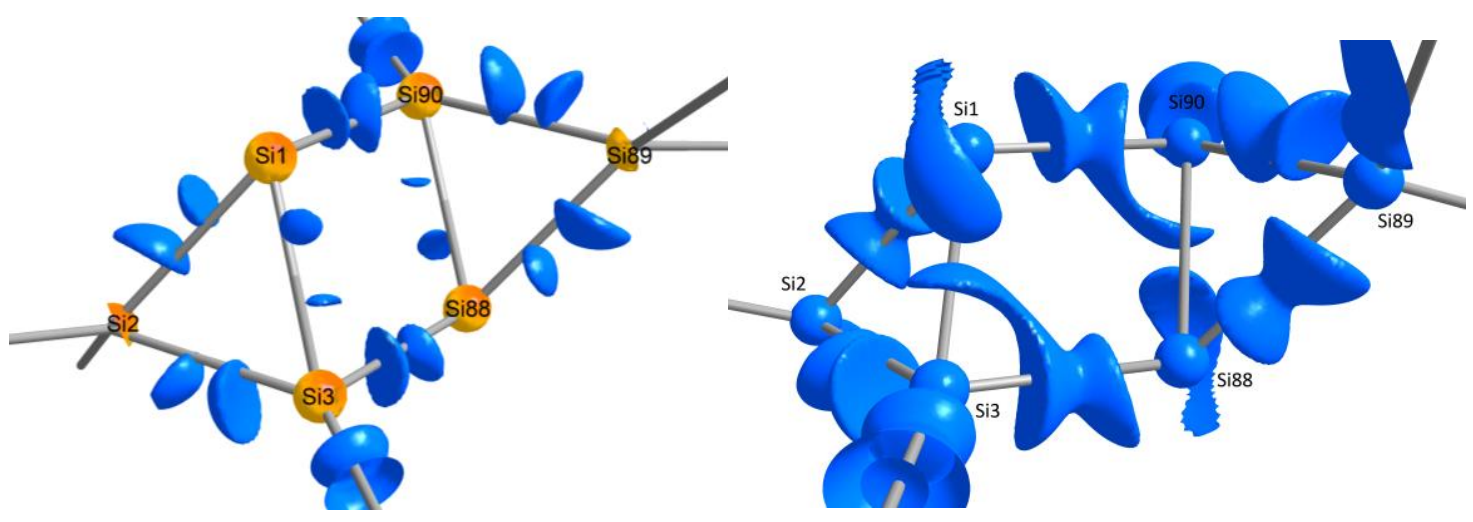

Figure 23 Laplacian distributions from theoretical data around the silicon atoms of $\mathbf{1}$ at an isosurface level of $-1.9 \mathrm{eA}^{-5}$ (left) and at $-1.34 \mathrm{eA}^{-5}$ level (right).

The distribution of $L(\boldsymbol{r})$ in Figure 23 shows a much more symmetric picture than the experimental results. The VSCCs for the Si1-Si90 and Si3-Si88 bonds are also more distinct than those of the transannular bonds but more straight directed to each other. The nonbonding VSCC in Figure 23 right appears at much lower absolute isolevel and hardly separates from the transannular VSCC (Figure 23 right). A reason for the more symmetric behavior in the theoretical data might be that the gas phase geometry is more symmetric than the crystal symmetry. The bonding, in the experiment, between $\mathrm{Si} 1$ and $\mathrm{Si} 2 / 3$ has similarities to a bifurcated hydrogen bond where the density of one donor atom is shared between two acceptor hydrogen atoms, ${ }^{[153-154]}$ or in newer publications with nitrogen as donor atom. ${ }^{[155-157]}$

\subsubsection{Electron Density at the Bond Critical Points}

The values of $\rho(\boldsymbol{r})$ at the BCP of the Si1-Si2 and Si1-Si3 bonds are similar with 0.537(5) [0.524] and $0.545(6)$ [0.518] $\AA^{-3}$, respectively, whereas the transannular Si2-Si3 bond accumulates only $0.466(7)$ [0.484] e $\AA^{-3}$. The highest value of $\rho\left(\boldsymbol{r}_{B C P}\right)$ is found between the Si2-Si3' silicon atoms with $0.595(11)$ [0.537] $\mathrm{e}^{-3}$. These findings fit to the values of $L(\boldsymbol{r})$ at the bond critical points. The stronger Si2-Si3'-bond accumulates the most density and the transannular bond the least. The plots of the static deformation density in Figure 26 and Figure 27 also support this picture. Remarkably is the accumulation of density in the tricyclic parts, as the density of atom Si1 interacts strongly with the bond density of the transannular bond.

\subsubsection{The Laplacian along the Bond Paths}

The same as for $\rho\left(\boldsymbol{r}_{B C P}\right)$ is valid for the $L\left(\boldsymbol{r}_{B C P}\right)$ values. Between Si1-Si2 and Si1-Si3 $L(\boldsymbol{r})$ is similar with $-1.645(8)[-1.798] \mathrm{e} \AA^{-5}$ and $-1.628(8)[-1.616] \mathrm{e} \AA^{-5}$, respectively, while the transannular Si2-Si3 bond shows a much higher $L(\boldsymbol{r})$ value of $-1.164(9)[-0.940]$ e $\AA^{-5}$. The most negative $L(\boldsymbol{r})$ for a Si-Si bond in 1 was found for the Si2-Si3' bond with -2.285(12) [-1.798] e $\AA^{-5}$. 

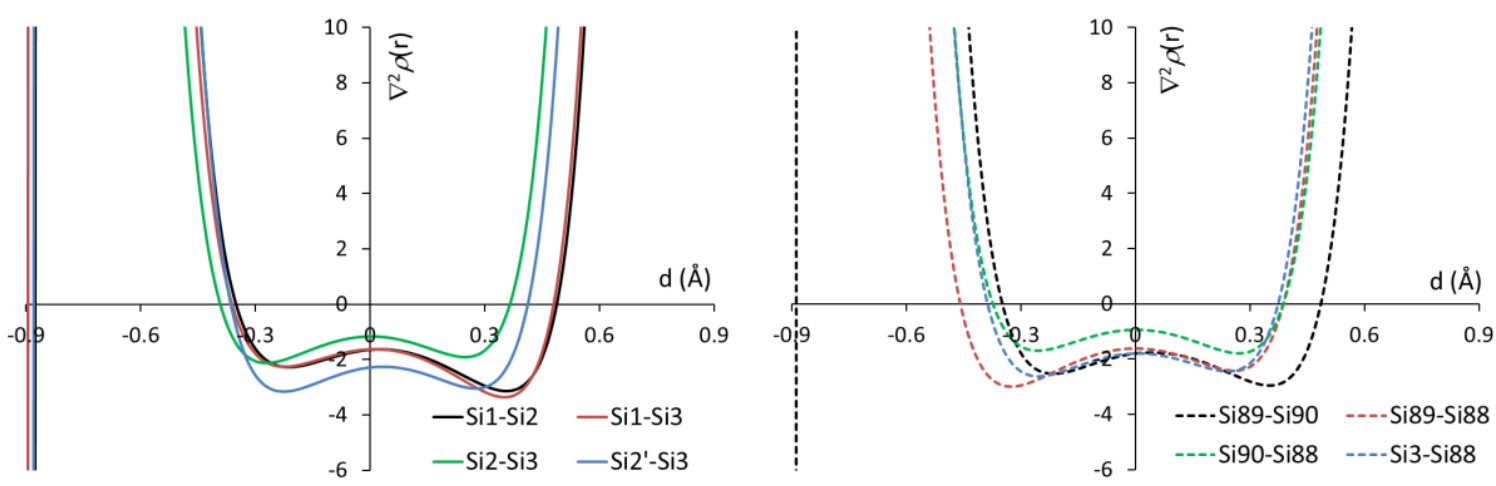

Figure 24 Distribution of $\boldsymbol{L}(\boldsymbol{r})$ along selected Si-Si bond paths from experimental data (left) and theory (right). The same color indicated equivalent bonds, see chapter 10.5.2 for numbering scheme.

The qualitative comparability for theory and experiment of the $L(\boldsymbol{r})$ distribution is remarkably good, except for Si1-Si3 (Figure 24). The weakest interaction is the transannular bond between Si2 and $\mathrm{Si} 3$, the strongest is in the four-membered ring between $\mathrm{Si}^{2}$ and $\mathrm{Si} 3$, accommodating the $\pi$ bond in the Lewis diagram, while the Si1-Si3 single bond in the three-membered ring lies half-way in between.

In contrast to the covalent silicon-silicon bonds, the silicon-carbon (Figure 25) bonds are much more polar. Close to the silicon atom $L(\boldsymbol{r})$ reaches a high maximum around $+600 \mathrm{e}^{-5}$ and decreases to almost zero at the BCP with a plateau of slightly negative values and reaches a minimum close to the carbon atoms. This distribution can be attributed to the strongly polar character of these bonds. The distribution of $L(\boldsymbol{r})$ also explains the differences of the value at the BCP between experiment and theory. A slight change in the position of the BCP makes a huge difference in the $L(\boldsymbol{r})$ value.
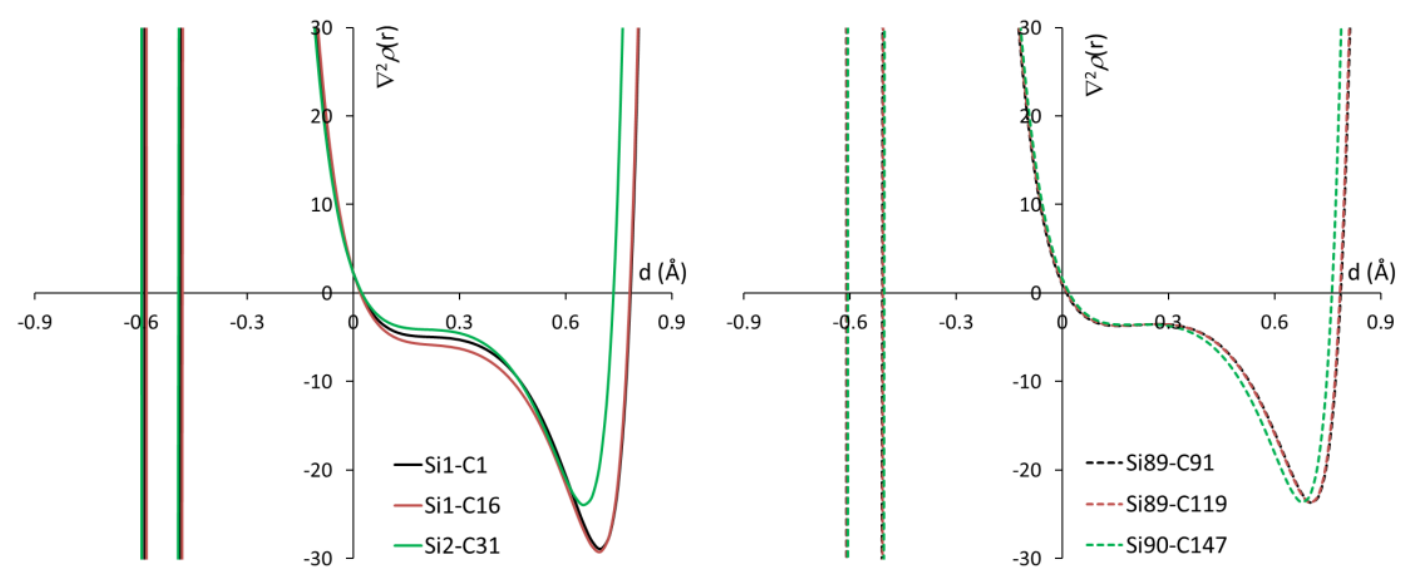

Figure 25 Laplacian distribution along selected $\mathrm{Si}-\mathrm{C}$ bond paths from experimental data (left) and theory (right). The same color indicated equivalent bonds, see chapter 10.5.2 for numbering scheme.

The comparison of experiment and theory in Figure 25 also documents a remarkably good agreement. 


\subsubsection{Static Deformation Density Maps of 1}
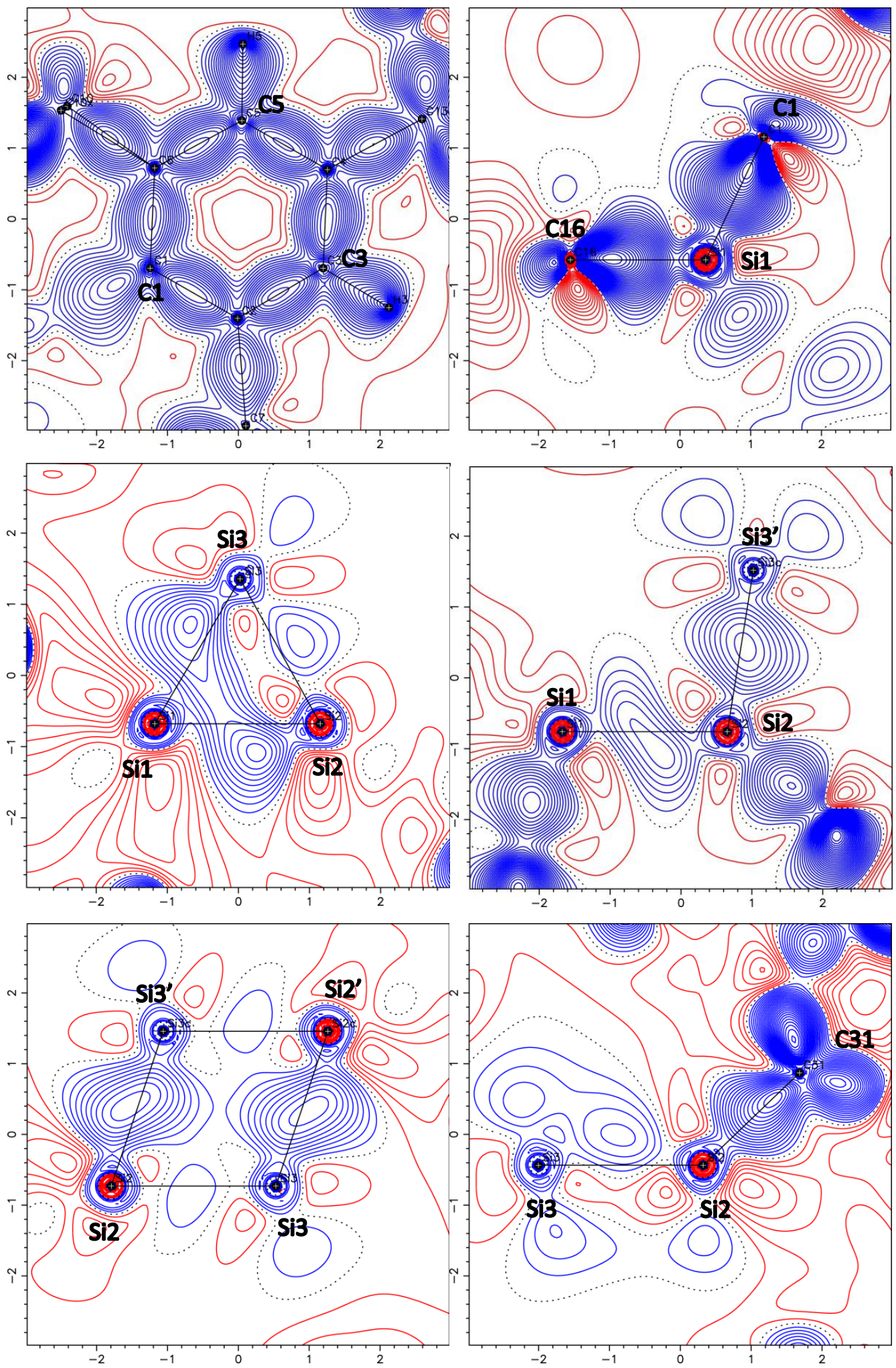

Figure 26 Contour plots of static deformation density of 1 . Contour lines are drawn at $\pm 0.02,0.04, \ldots$ e $\AA^{-3}$ level (first plot at $0.04 \mathrm{e}^{-3}$ ). 


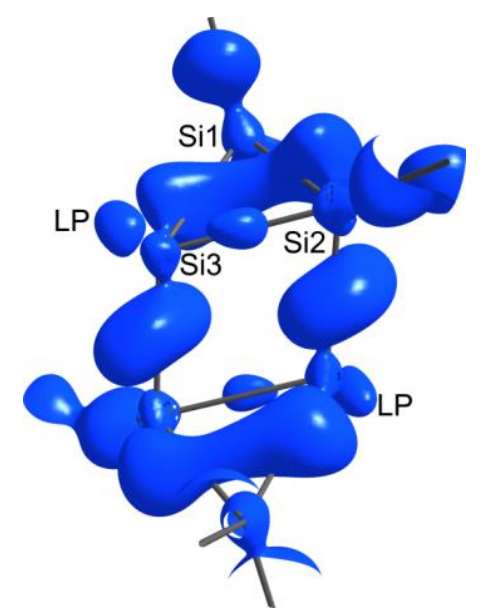

Figure 27 Isosurface representation of the static deformation density of 1 at $0.07 \mathrm{e}^{-3}$ level.

Depicted in Figure 26 are several plots of the static deformation density in different planes of the molecule 1. Figure 27 shows a three dimensional isosurface representation of the static deformation density. The Si-Si bonds in the tricyclic parts of 1 show clear indications of bent bonds with the electron density out of the straight line between two atoms. This behavior is much less pronounced in the Si2-Si3' and Si2'-Si3 bonds but the deformation density in Figure 27 shows a broad density distribution in these two bonds. This originates possibly from the rhomboid shape of the four cyclic ring of Si2-Si3-Si2'-Si3'.

\subsubsection{Ellipticity along the Bond Path}
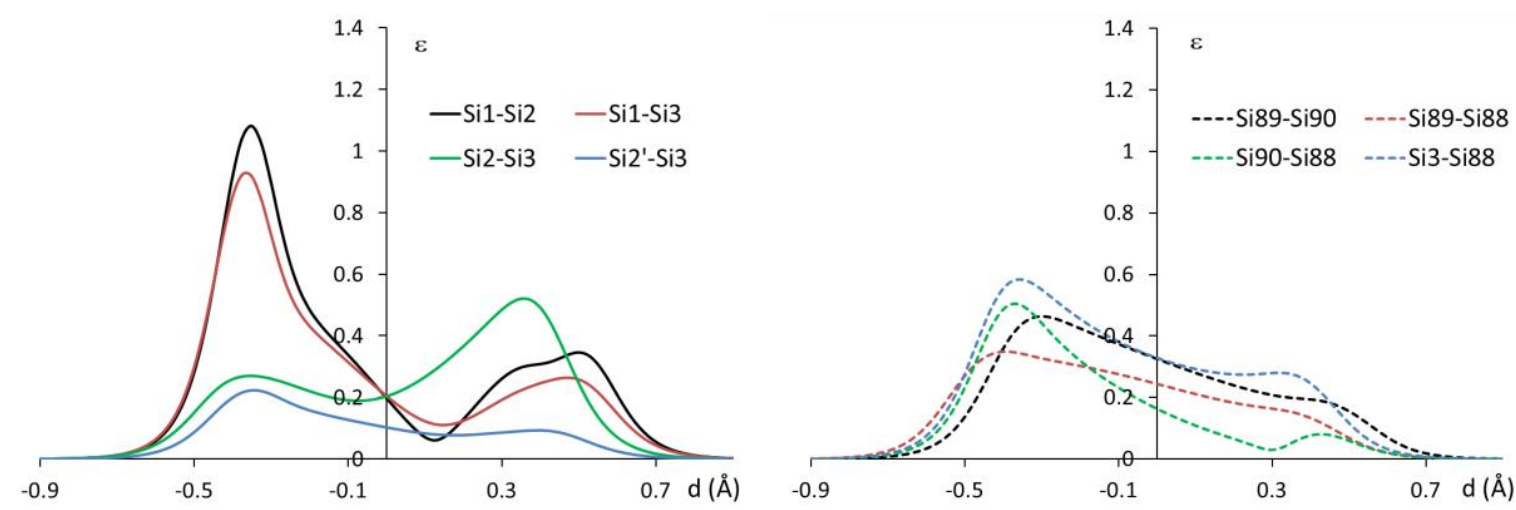

Figure 28 Distribution of $\epsilon$ along the Si-Si bond paths from experimental data (left) and theory (right). The same color indicated equivalent bonds, see chapter 10.5.2 for numbering scheme.

The distribution of the ellipticity in Figure 28 shows a generally higher ellipticity for the Si-Si bonds in comparison to the $\sigma-\mathrm{C}-\mathrm{Si}$ bonds. Especially near $\mathrm{Si} 1 \epsilon$ reaches a high level. This peak in $\epsilon$ might be caused by the special binding of Si1 to Si2 and Si3 discussed in chapter 5.3.2. 

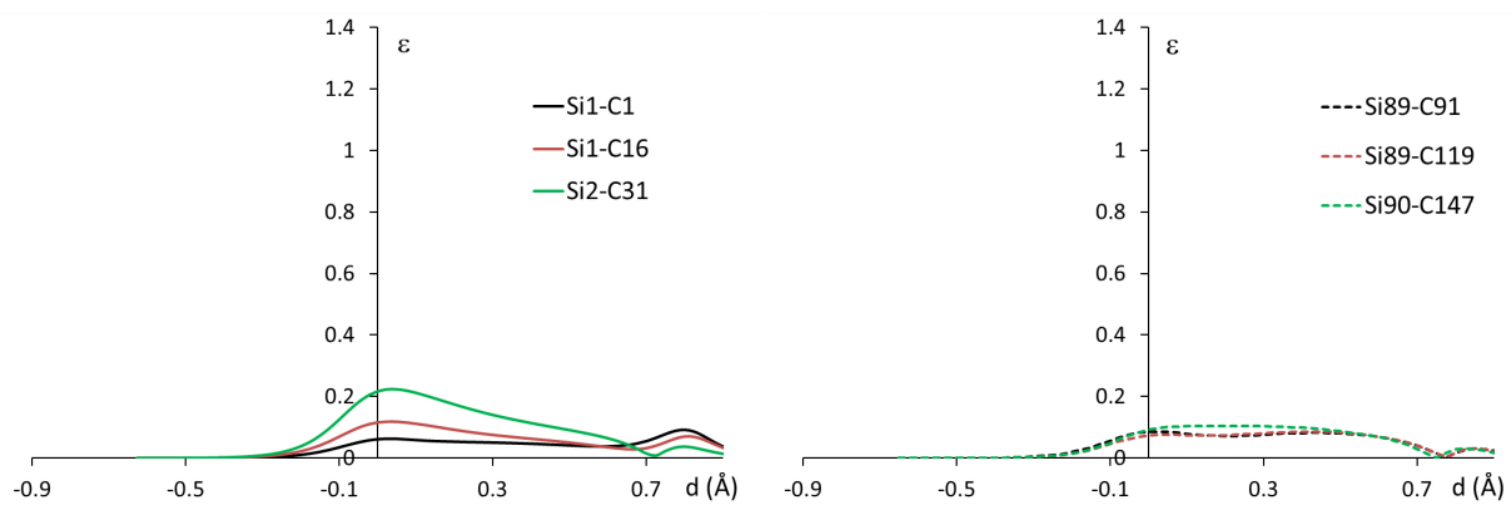

Figure 29 Distribution of $\epsilon$ along the $\mathrm{Si}-\mathrm{C}$ bond paths from experimental data (left) and theory (right). The same color indicated equivalent bonds, see chapter 10.5.2 for numbering scheme.

The experimentally derived ellipticity along the $\mathrm{Si}-\mathrm{C}$ bonds is low, as expected for $\sigma$-bonds, and is comparable to the theory.

\subsubsection{Residual Density Distribution}

Table 8 shows the highest peaks and deepest holes in the residual density distribution and their distance to the next atom after multipole refinement.

Table 8 Results from the Fast Fourier Transformation (xdfft, no cutoff) after final refinement of 1.

\begin{tabular}{lrllrllr} 
peak & distance $[\AA ̊$ & & & & & \multicolumn{2}{c}{$\begin{array}{l}\text { height } \\
{\left[\mathrm{e}^{-3}\right]}\end{array}$} \\
\hline $\mathrm{PK}(1)$ & 0.58 & from & $\mathrm{C}(55)$ & 0.7873 & 0.0478 & 0.6255 & 0.44 \\
$\mathrm{PK}(2)$ & 0.53 & from & $\mathrm{Si}(2)$ & 0.5780 & 0.0256 & 0.1354 & 0.40 \\
$\mathrm{PK}(3)$ & 0.55 & from & $\mathrm{C}(42)$ & 0.4724 & -0.0653 & 0.3000 & 0.37 \\
$\mathrm{PK}(4)$ & 0.53 & from & $\mathrm{Si}(1)$ & 0.5227 & 0.0964 & 0.0379 & 0.36 \\
\hline $\mathrm{HL}(1)$ & 0.46 & from & $\mathrm{C}(51)$ & 0.8023 & 0.1338 & 0.5492 & -0.39 \\
$\mathrm{HL}(2)$ & 0.40 & from & $\mathrm{C}(50)$ & 0.7807 & 0.1046 & 0.5615 & -0.39 \\
$\mathrm{HL}(3)$ & 0.19 & from & $\mathrm{C}(42)$ & 0.4696 & -0.0714 & 0.2755 & -0.27 \\
$\mathrm{HL}(4)$ & 0.47 & from & $\mathrm{C}(11)$ & 0.6230 & 0.2377 & 0.1723 & -0.26 \\
\hline
\end{tabular}
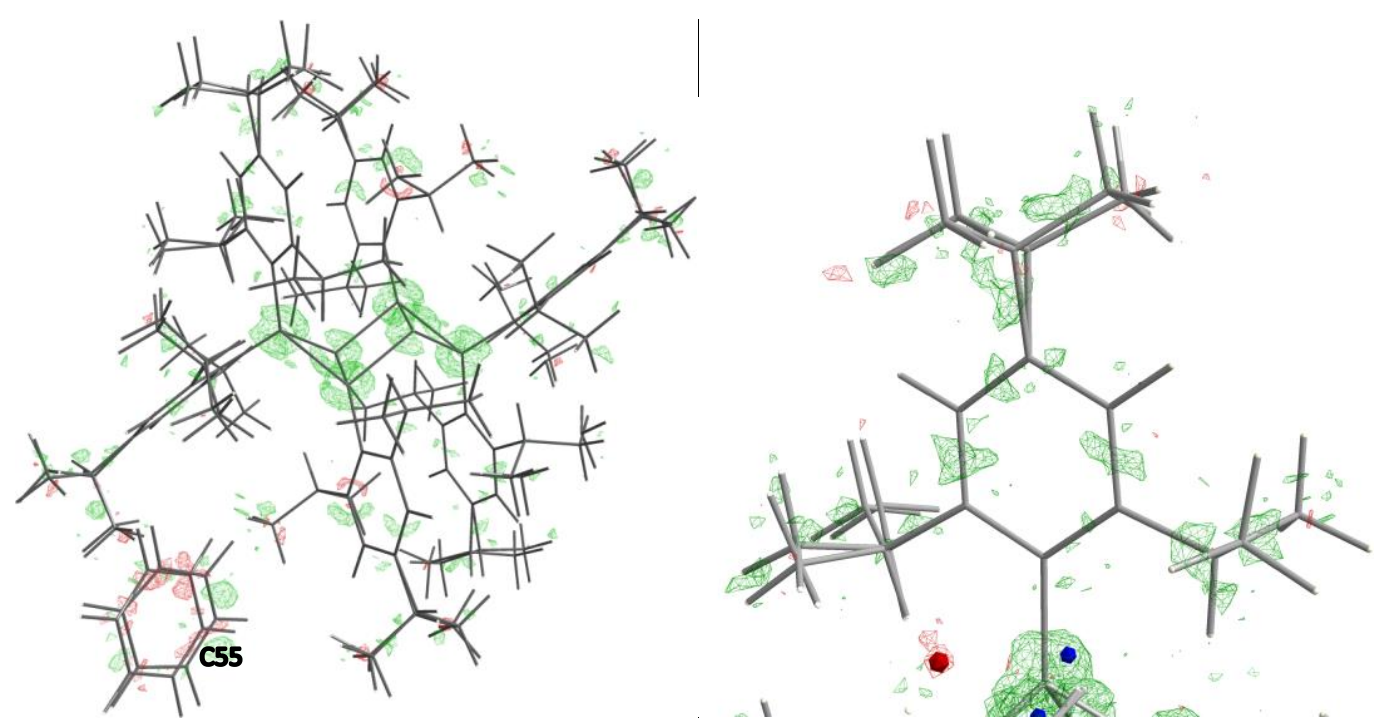

Figure 30 Left: Residual density analysis of 1 after final refinement cycle (all data, contour value $=0.14 \mathrm{e}^{-3}$ ). Right: Residual density analysis of one Tip group (all data, contour value $=0.11 \mathrm{e}^{-3}$ ). 
The highest residual peak is located $0.58 \AA$ next to $\mathrm{C} 55\left(0.44 \mathrm{e} \AA^{-1}\right)$ of the benzene solvent molecule. Because of the lack of restraints in XD2006 and the suspected dynamic disorder this could not be modeled better. The residual density around the silicon atoms has ring-like features. It was not possible to lower the residual density around the silicon atoms with different zeta values so it was left at the default value of the Su, Coppens, Macchi data bank file included in XD2006.

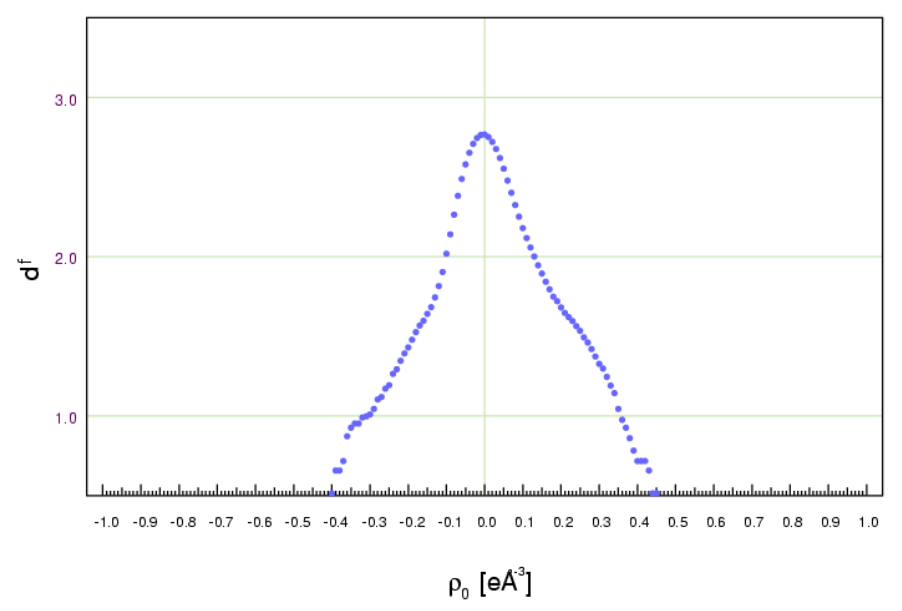

Figure 31 Fractal dimension versus residual density in structure $1^{[48]}$

Inspection of the residual density with jnk2RDA (chapter 3.3.5) results in the distribution of the fractal dimension seen in Figure 31. ${ }^{[4]}$ The deviation from ideal Gaussian shape originates mainly from the non-ideal description of the solvent molecules and the isopropyl groups of the Tip ligands. Nonetheless, the multipole refinement was successful because of the high data to parameter ratio (chapter 5.2.3) and yielded a satisfactorily residual density even in the disordered regions of the Tip groups. It shows that an invariom model is capable to describe the ligand periphery successfully. 


\subsection{Topological Analysis of the Hexasilabenzene Isomer 2}

The following electron density analysis is focused on the properties that can be derived from experimental data. The theoretical details such as NMR resonance and density functional calculations were previously analyzed by Scheschkewitz et al. ${ }^{[108]}$ Nevertheless, all property values were compared to the respective values derived from the multipole refinement against calculated structure factors obtained from Fourier transform with the program TONTO ${ }^{[140-141]}$ from a previously calculated single-point wave functions (Gaussian ${ }^{[142]}$ at the $\omega B 97 X D / 6-311 G(d, p)$ level) ${ }^{[106,108]}$ All values which are compared to the values obtained from the refinements against calculated structure factors (with TONTO ${ }^{[141]}$ ) are given in brackets. Since the outer sphere of $\mathbf{2}$ was modeled by invarioms, no information on the topology of the electron density for these part of the molecules can be extracted. Therefore, the following study is only focused on the Si-Si and Si-C bond of the molecule. The second investigated isomer of hexasilabenzene $\mathbf{2}$ crystallizes in the space group $\mathrm{C} 2 / \mathrm{c}$ with $\mathrm{Si} 1$ and $\mathrm{Si} 4$ on a twofold rotational axis. The asymmetric unit contains half a molecule and 1.5 THF molecules as lattice solvent. The bridged propellane-like $\mathbf{2}$ adopts a cage structure consisting of six silicon atoms. As in 1, two vertices are substituted by two Tip ligands, two by one and two by none, but bound to just three silicon atoms.

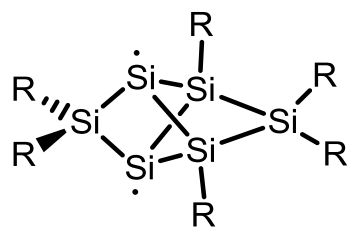<smiles></smiles>

Scheme 25 Structure 1 (left) and [1.1.1]propellane (right). R = Tip, 2,4,6-triisopropylphenyl.

\subsubsection{Bond Critical Points and Molecular Graph of 2}

A formally correct Lewis diagram of $\mathbf{2}$ can only be drawn as diradical or with a bond between the bridgehead atoms to fulfill the octet rule and the fourfold valency of carbon. This immediately brings up the question if there is a bond between the bridgehead atoms ( $\mathrm{Si} 3$ and $\mathrm{Si}^{\prime}$ ).

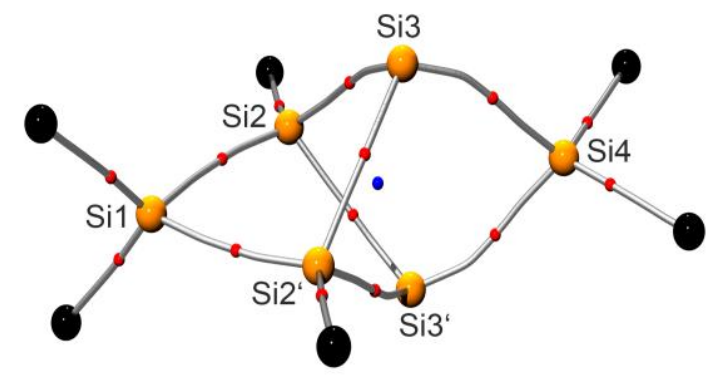

Figure 32 Molecular graphs of 2, depicting the curved bond paths, bond critical points (red) and ring critical point (blue).

Table 9 Topological parameters of the BCP and integrated atomic charges of $\mathbf{2}$ [theoretical values in brackets]. 


\begin{tabular}{|c|c|c|c|c|c|}
\hline bond $^{[a]}$ & $\rho(\mathbf{r})\left[\mathrm{e} \AA^{-3}\right]$ & $\nabla^{2} \rho(\mathbf{r})\left[\mathrm{e} \AA^{-5}\right]$ & $\epsilon_{\mathrm{BCP}}$ & $\eta_{\mathrm{BCP}}$ & $\mathrm{Q}[\mathrm{e}]$ \\
\hline Si1*-Si6 & $0.580(5)[0.575]$ & $-2.942(8) \quad[-2.580]$ & $0.13[0.08]$ & $2.60[2.17]$ & $+1.06[+1.45]$ \\
\hline $\mathrm{Si} 2 *-\mathrm{Si} 3^{\prime}$ & $0.555(9) \quad[0.538]$ & $-1.895(10)[-1.783]$ & $0.64[0.29]$ & $1.80[1.55]$ & $+0.73[+0.64]$ \\
\hline Si2-Si3* & $0.512(4) \quad[0.532]$ & $-1.586(7) \quad[-1.749]$ & $0.49[0.29]$ & $1.47[1.50]$ & $-0.30[-0.15]$ \\
\hline Si3-Si4* & $0.527(4) \quad[0.519]$ & $-1.925(4) \quad[-1.685]$ & $0.38[0.29]$ & $1.63[1.43]$ & $+1.55[+1.41]$ \\
\hline Si1-C1* & $0.771(5) \quad[0.763]$ & $+2.822(8) \quad[+2.052]$ & $0.04[0.07]$ & $0.36[0.40]$ & $-0.64[-0.70]$ \\
\hline Si2-C16* & $0.776(7) \quad[0.785]$ & $+2.645(16)[+2.512]$ & $0.24[0.07]$ & $0.40[0.38]$ & $-0.67[-0.73]$ \\
\hline $\mathrm{Si} 4-\mathrm{C} 31^{*}$ & $0.775(5) \quad[0.776]$ & $+1.569(8) \quad[+0.950]$ & $0.16[0.07]$ & $0.45[0.46]$ & $-0.61[-0.71]$ \\
\hline
\end{tabular}

$\mathrm{Q}$ : the charge of the $\left({ }^{*}\right)$-marked atom, derived by the difference of atomic number $Z$ and $\boldsymbol{\rho}(\boldsymbol{r})$ integrated over the respective atomic basin.

Different to the carbon-based [1.1.1]propellane there is no accumulation of electron density (Figure 33) and no indication of a $\mathrm{Si}-\mathrm{Si}$ bond path between the bridgehead atoms $\mathrm{Si} 3 \cdots \mathrm{Si} 3$ ' at the hub of the silapropellane moiety. ${ }^{[117]}$ Like in 1 , there are distinct VSCCs in the non-bonding regions of Si3 and Si3' $\left(-4.00[-1.15]\right.$ e $\left.\AA^{-5}\right)$ pointing away from the inner silicon cage (Figure 37$)$ in line with the expectation derived from the simple Lewis formalism (Scheme 25).

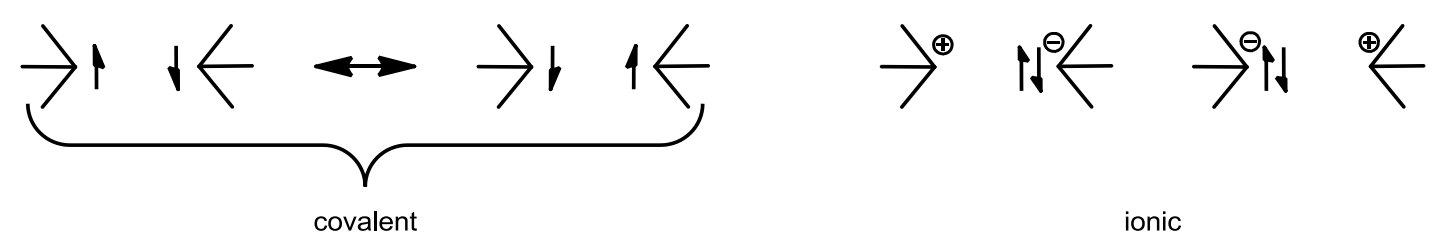

Scheme 26 Valence bond structures that describe a charge-shift bond.

In the homologous [1.1.1]propellanes the existence of a central bond is, amongst other things, explained by Shaik et al. with the existence of a charge shift bond (Scheme 26). ${ }^{[158-159]}$ The hybrid atomic orbitals located at the bridge-head of [1.1.1]propellane are outwardly directed, which is in agreement with the general consensus that there is a degree of hybridization lying in between the classical $\mathrm{sp}$ and $\mathrm{sp}^{2}$ hybrid orbitals. In valence bond theory and in the chemist's language, a bond is regarded as an interaction between two localized hybrid atomic orbitals. In the homonuclear case (as in [1.1.1]propellane), this interaction always takes the form of a combination of a major covalent structure and two minor ionic ones (Scheme 26). This bond type is a non-classical type of bonding in which the covalent-ionic resonance energy plays the major role. A typical characteristic is that the electron density is depleted in the middle of the bond and thus at the bond critical point, a positive $L(\boldsymbol{r})$ is found. A bond is defined as charge shifted when the resonance energy arising from the mixing of covalent and ionic structures $\left(R E_{c-i}\right)$, defined as the energy difference between the ground-state and the major valence bond structure, contributes more than $50 \%$ to the overall bonding energy. 


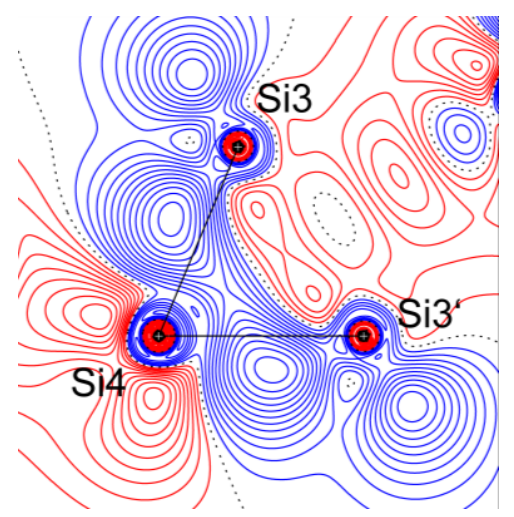

Figure 33 Static deformation density contour plot of 2. Contour lines are drawn at $\pm 0.015,0.03, \ldots e \AA^{-3}$ interval level; blue: positive; red: negative values.

The non-existence of a bridgehead bond path confirms a substantial biradical character, but the closeness of a ring critical point to the potential bond critical point (blue point in Figure 32, and Figure 36) also confirms that even small differences in the density of these systems can create or prevent a bridgehead bond path. ${ }^{[160]}$
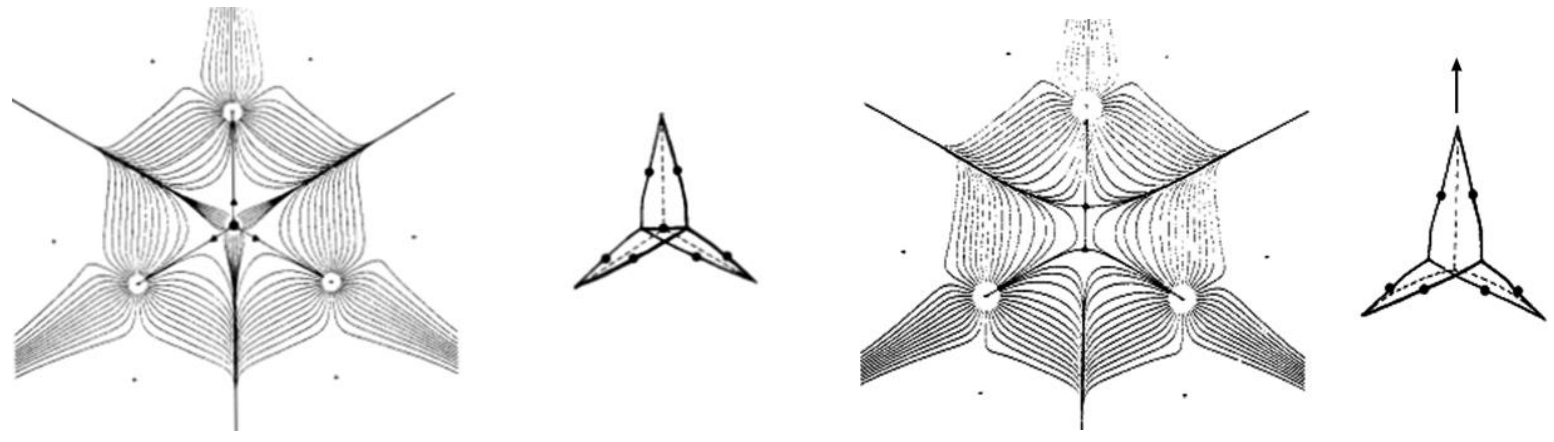

Figure 34 Maps of the gradient vector field in the $\sigma_{h}$-plane of [1.1.1]propellane and the corresponding molecular graph. broken lines: profiles of the interatomic surfaces. Left: undistorted molecule with central bond path. Right: distorted molecule without central bond path. ${ }^{[161]}$

By comparison of this work with the results from Bader et al. (1981) it became even more clear that slight distortion of the geometry of [1.1.1]propellanes can create or prevent a central bond path. ${ }^{[161]}$ The structure in Figure 34 (right) illustrates the change in the gradient vector field when one apical carbon atom is being displaced. The central bond path vanishes, and its bond critical point changes into a pair of ring critical points (Figure 35). 


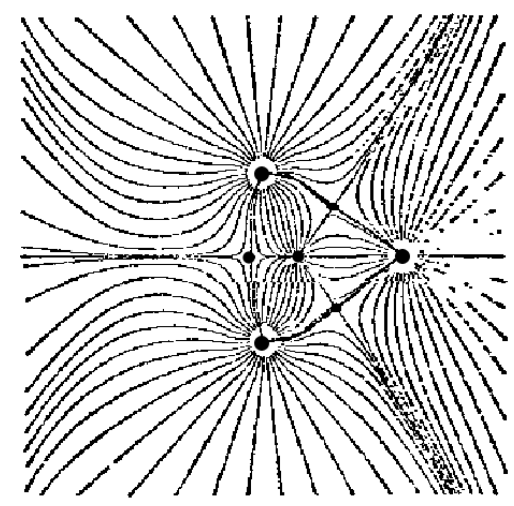

Figure 35 Map of the gradient vector field for $\mathrm{C}_{5} \mathrm{H}_{6}$ in the $\sigma_{\mathrm{v}}$-plane containing the two bridgehead carbons and one apical carbon. ${ }^{[161]}$

It is remarkable that the gradient vector field for $\mathbf{2}$ contains a similar picture to the findings of Bader et al. for a distorted [1.1.1]propellane where one apical carbon atom is displaced in a way such that its bonds are elongated. In Figure 36 right the two ring critical points similar to Figure 35 appear in the triangle of Si4-Si3-Si3'. In Figure 36 left, there is again a similar behavior to the propellane in Figure 34 with two ring critical points. The third $(3,-1)$ point results from the bridging $\mathrm{Si} 4$ atom. A second influence on the gradient vector field originates from the hydrogen atoms of the isopropyl groups near $\mathrm{Si} 3$ and $\mathrm{Si}^{\prime}$. This results in the formation of bond path and a cage critical point (Figure 36, left). The formation of bond path to the bridgehead atoms might be a reason for the relatively high chemical stability of compound $\mathbf{2}$.

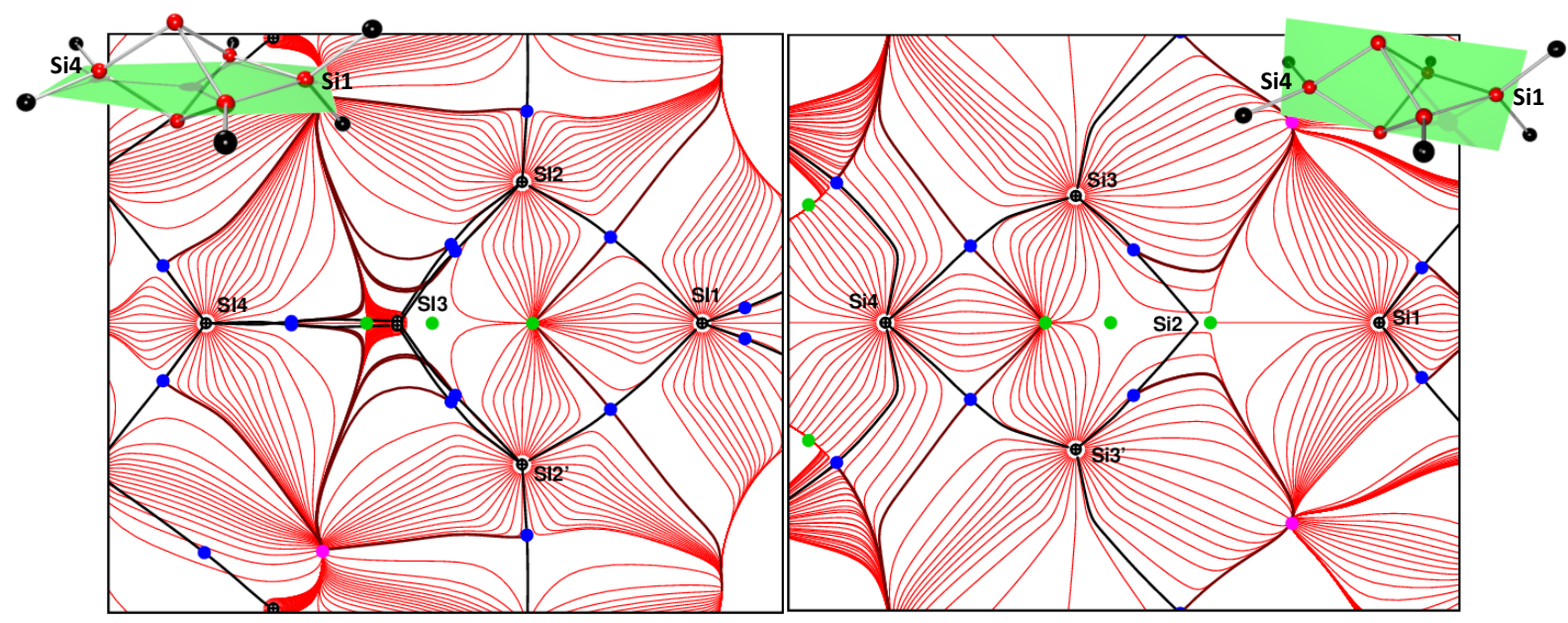

Figure $\mathbf{3 6}$ Gradient vector field of $\mathbf{2}$ in two perpendicular planes. Bond critical points: blue, ring critical points: green, cage critical points: purple. 


\subsubsection{Integrated Charges and Valence Shell Charge Concentrations}

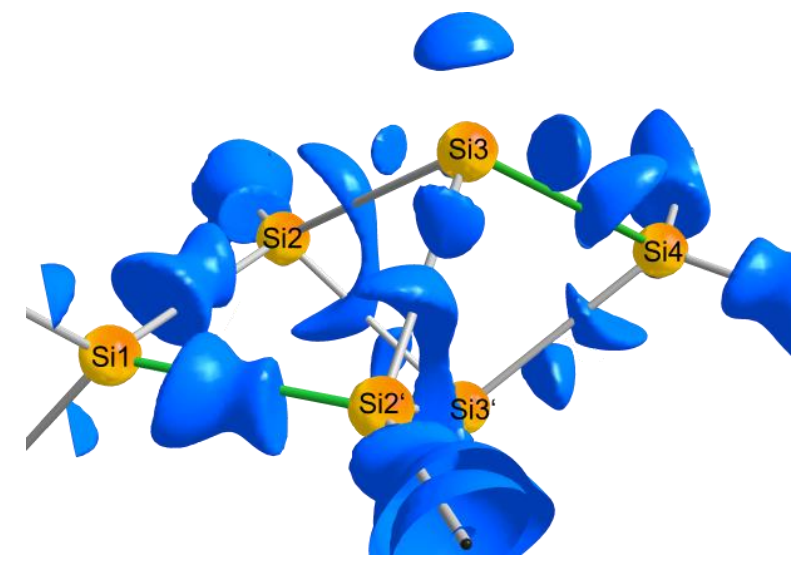

Figure $\mathbf{3 7}$ Laplacian distribution from experimental data around the silicon atoms of $\mathbf{2}$ at an isosurface level of $-1.9 \mathrm{e} \AA^{-5}$.

As the conformational change from $\mathbf{1}$ to $\mathbf{2}$ would not require any ligand scrambling in the cage structure 2 the expected oxidation states of the silicon atoms are $+\mathrm{II}$ for Si1 and Si4, $+\mathrm{I}$ for Si2 and zero for Si3 like in $\mathbf{1}$. The integration of the experimental density resulted in a similar pattern with a slight difference to the theoretical data. Si1 and Si4 differ clearly in their experimentally derived value of 1.06 [1.45] e (Si1) and 1.55 [1.41] e (Si4). In fact, the differing charges are in better agreement with chemistry than the theory. Si1 and Si4 show different reactivity of $\mathbf{2}$ as recently reported by the group of Scheschkewitz. ${ }^{[136]}$ However, the high integrated charge of Si4 also fits the unusual low field resonance of $\delta=174.6 \mathrm{ppm}$ in the ${ }^{29} \mathrm{Si} \mathrm{NMR} \mathrm{spectrum} \mathrm{of} 2$ described earlier, and it can be rationalized by invoking magnetically induced cluster currents. ${ }^{[108]} \mathrm{Si2}$ and $\mathrm{Si3}$ show much lower Bader charges (+0.73 e [+0.64 e] and -0.30 e [-0.15 e]).

The valence shell charge concentrations in Figure 37 show that after conversion from $\mathbf{1}$ to $\mathbf{2}$ the charge concentrations of Si1 to Si2 are more directly pointed to the atoms and not to the bond density as in 1 . The VSCC at Si3 has a very round shape. In contrast to are the VSCCs at Si2 and Si4, their charge concentrations in the direction of $\mathrm{Si} 3$ and $\mathrm{Si}^{\prime}$ ' is more smeared out and even merges for two VSCCs in Figure 37. This documents a delocalization of density in the propellane moiety.

Table 10 Integrated charges and VSCCs at selected atoms in $\mathbf{2}$ (experimental data).

\begin{tabular}{|c|c|c|c|c|c|}
\hline & \begin{tabular}{|l} 
Integration \\
$\mathrm{Q}[\mathrm{e}]$
\end{tabular} & $\begin{array}{l}\text { integrated } \\
\text { Lagrangian }\end{array}$ & $\begin{array}{l}\text { VSCC } \\
\nabla^{2} \rho(\mathbf{r})\left[\mathrm{e} \AA^{-5}\right]\end{array}$ & distance $[\AA]]$ & $\begin{array}{l}\text { VSCC } \\
\text { direction }\end{array}$ \\
\hline \multirow[t]{2}{*}{ Si(1) } & 1.06 & $-3.17 \cdot 10^{-3}$ & -4.36 & 0.911 & $\mathrm{Si} 2$ \\
\hline & & & -4.36 & 0.911 & $\mathrm{Si}^{\prime}$ \\
\hline \multirow[t]{3}{*}{ Si(2) } & 0.73 & $2.12 \cdot 10^{-4}$ & -3.47 & 0.914 & Si3' \\
\hline & & & -3.46 & 0.928 & Si1 \\
\hline & & & -2.84 & 0.921 & $\mathrm{Si} 3$ \\
\hline \multirow[t]{4}{*}{ Si(3) } & -0.30 & $-4.21 \cdot 10^{-4}$ & -2.41 & 0.922 & $\mathrm{Si} 2$ \\
\hline & & & -3.37 & 0.913 & $\mathrm{Si} 4$ \\
\hline & & & -2.55 & 0.925 & $\mathrm{Si}^{\prime}$ \\
\hline & & & -4.00 & 0.904 & LP \\
\hline \multirow[t]{3}{*}{ Si(4) } & 1.55 & $1.80 \cdot 10^{-3}$ & -3.15 & 0.921 & $\mathrm{Si} 3$ \\
\hline & & & -4.86 & 0.948 & C31 \\
\hline & & & -3.15 & 0.921 & $\mathrm{Si}^{\prime}$ \\
\hline \multirow[t]{3}{*}{$C(1)$} & -0.64 & $-7.90 \cdot 10^{-4}$ & -27.50 & 0.484 & C6 \\
\hline & & & -30.18 & 0.481 & $\mathrm{C} 2$ \\
\hline & & & -30.97 & 0.479 & Si1 \\
\hline
\end{tabular}




\begin{tabular}{l|ll|ll|l}
\hline $\mathbf{C ( 1 6 )}$ & $\mathbf{- 0 . 6 7}$ & $6.77 \cdot 10^{-5}$ & $\mathbf{- 2 9 . 9 6}$ & 0.482 & $\mathrm{C} 21$ \\
& & & $\mathbf{- 2 6 . 7 5}$ & 0.483 & Si2 \\
& & & $\mathbf{- 2 8 . 5 0}$ & 0.484 & $\mathrm{C} 17$ \\
\hline $\mathbf{C ( 3 1 )}$ & $\mathbf{- 0 . 6 1}$ & $-3.25 \cdot 10^{-3}$ & $\mathbf{- 2 9 . 8 5}$ & 0.482 & $\mathrm{C32}$ \\
& & & $\mathbf{- 2 7 . 4 6}$ & 0.483 & Si4 \\
& & & $\mathbf{- 2 9 . 1 2}$ & 0.482 & $\mathrm{C} 36$ \\
\hline
\end{tabular}

Table 11 Integrated charges and VSCCs at selected atoms in $\mathbf{2}$ (theoretical data).

\begin{tabular}{|c|c|c|c|c|c|}
\hline & \begin{tabular}{|l} 
Integration \\
$\mathrm{Q}[\mathrm{e}]$
\end{tabular} & $\begin{array}{l}\text { integrated } \\
\text { Lagrangian }\end{array}$ & $\begin{array}{l}\text { VSCC } \\
\nabla^{2} \rho(\mathbf{r})\left[\mathrm{e} \AA^{-5}\right]\end{array}$ & distance $[\AA ̊]$ & \begin{tabular}{|l} 
VSCC \\
direction
\end{tabular} \\
\hline \multirow[t]{4}{*}{ 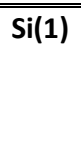 } & 1.45 & $1.29 \cdot 10^{-3}$ & -4.00 & 0.936 & C35 \\
\hline & & & -4.00 & 0.936 & C7 \\
\hline & & & -3.48 & 0.925 & Si2 \\
\hline & & & -3.48 & 0.925 & Si6 \\
\hline \multirow[t]{3}{*}{ Si(2) } & 0.64 & $2.47 \cdot 10^{-3}$ & -2.76 & 0.924 & $\mathrm{Si} 3$ \\
\hline & & & -3.53 & 0.922 & Si1 \\
\hline & & & -2.73 & 0.924 & Si5 \\
\hline \multirow[t]{4}{*}{ Si(3) } & -0.15 & $1.88 \cdot 10^{-3}$ & -1.15 & 0.929 & LP \\
\hline & & & -2.65 & 0.921 & $\mathrm{Si} 4$ \\
\hline & & & -2.56 & 0.922 & Si2 \\
\hline & & & -2.64 & 0.922 & Si6 \\
\hline \multirow[t]{4}{*}{$\mathrm{Si}(4)$} & 1.41 & $-2.25 \cdot 10^{-4}$ & -2.77 & 0.921 & $\mathrm{Si} 3$ \\
\hline & & & -4.15 & 0.914 & C91 \\
\hline & & & -4.15 & 0.914 & C119 \\
\hline & & & -2.77 & 0.921 & Si5 \\
\hline \multirow[t]{3}{*}{$\bar{C} C(7)$} & -0.70 & $4.63 \cdot 10^{-4}$ & -24.92 & 0.485 & Si1 \\
\hline & & & -25.11 & 0.487 & C14 \\
\hline & & & -25.66 & 0.486 & C8 \\
\hline \multirow[t]{3}{*}{$C(63)$} & -0.73 & $5.81 \cdot 10^{-4}$ & -25.36 & 0.487 & C64 \\
\hline & & & -25.36 & 0.487 & C70 \\
\hline & & & -24.41 & 0.486 & Si2 \\
\hline \multirow[t]{3}{*}{$C(91)$} & -0.71 & $1.12 \cdot 10^{-3}$ & -25.43 & 0.486 & C92 \\
\hline & & & -24.11 & 0.486 & C98 \\
\hline & & & -25.46 & 0.487 & $\mathrm{Si} 4$ \\
\hline
\end{tabular}

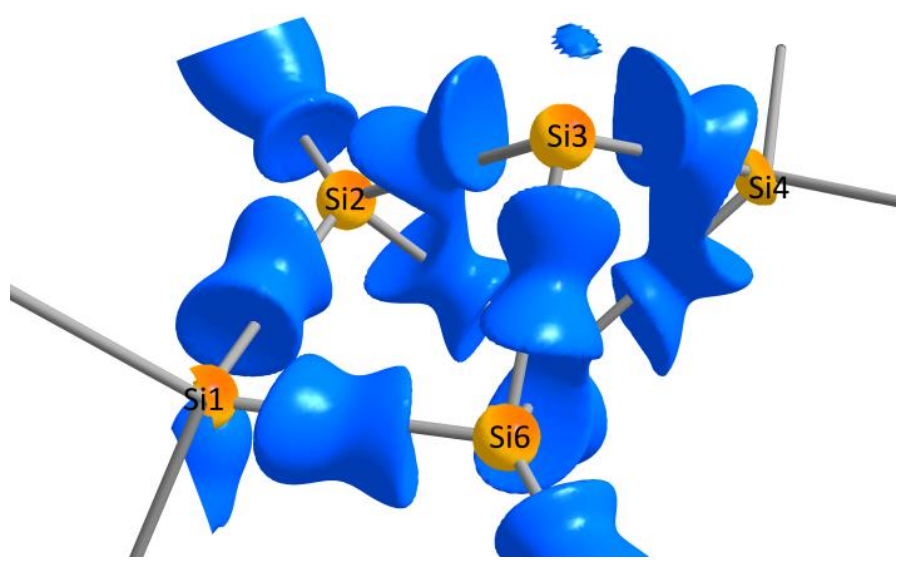

Figure 38 Laplacian distributions from theoretical data around the silicon atoms of $\mathbf{2}$ at an isosurface level of $-1.1 \mathrm{e}^{-5}$ level.

In comparison to Figure 37, the distribution of $L(\boldsymbol{r})$ in Figure 38 is much more symmetrically distributed. While the features of the $\mathrm{Si} 1-\mathrm{Si} 2$ and $\mathrm{Si} 3-\mathrm{Si} 4$ bonds are generally well comparable to 
the experiment, the VSCC above Si3 is at much smaller level. The merged VSCCs at Si2, Si6 and Si4 show that the density concentrations in the bonds to Si3 are not only pointing towards the Si3 alone. Especially the VSCCS of Si4 are more merged and not so well separated as in the experiment. Nevertheless, the VSCCS of the apical silicon atoms have a tendency to merge into the direction of the propellane center.

\subsubsection{Electron Density at the Bond Critical Point}

The electron density values at the bond critical point of the Si1-Si2 bond are upon conversion from 1 to 2, the highest values in 2 with 0.580 [0.575] e $\AA^{-3}$, followed by Si2-Si3' and Si2-Si3 with $0.555[0.538]$ and $0.512[0.532] \mathrm{e}^{-3}$, respectively. The Si3-Si4 bond lies in between with 0.527 [0.519] $\mathrm{e}^{-3}$. In terms of bond strength this picture is consistent with $L(\boldsymbol{r})$ at the BCP. Si1-Si2 has the strongest shared interaction with $-2.942[-2.580] \mathrm{e}^{-5}$ and the other BCPs show a much lower level of around $-1.8 \mathrm{e}^{-5}$ with the weakest interaction being the $\mathrm{Si} 2-\mathrm{Si} 3$ bond.

\subsubsection{The Laplacian along the Bond Paths}

The qualitative shapes of the $L(\boldsymbol{r})$ curves in $\mathbf{2}$ are comparable to $\mathbf{1}$, but the shape of the four curves for $\mathbf{2}$ is very similar. This is a hint that charge is more evenly distributed in the silicon cage. The silicon-carbon bonds in $\mathbf{2}$ also shows the strong polarization towards the carbon atoms (Figure 41) with a low $\eta$ value (Table 9, chapter 5.4.1) and thus high ionic contribution. ${ }^{[162-163]}$

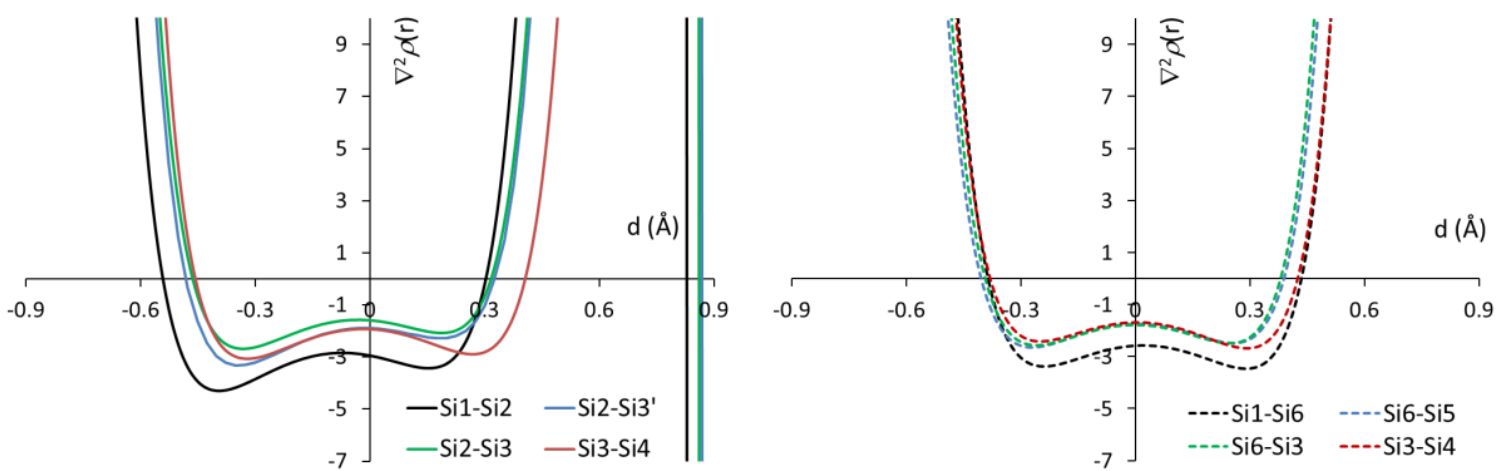

Figure 39 Distribution of $\boldsymbol{L}(\boldsymbol{r})$ along selected Si-Si bond paths from experimental data (left) and theory (right). The same color indicated equivalent bonds, see chapter 10.5.3 for numbering scheme. 

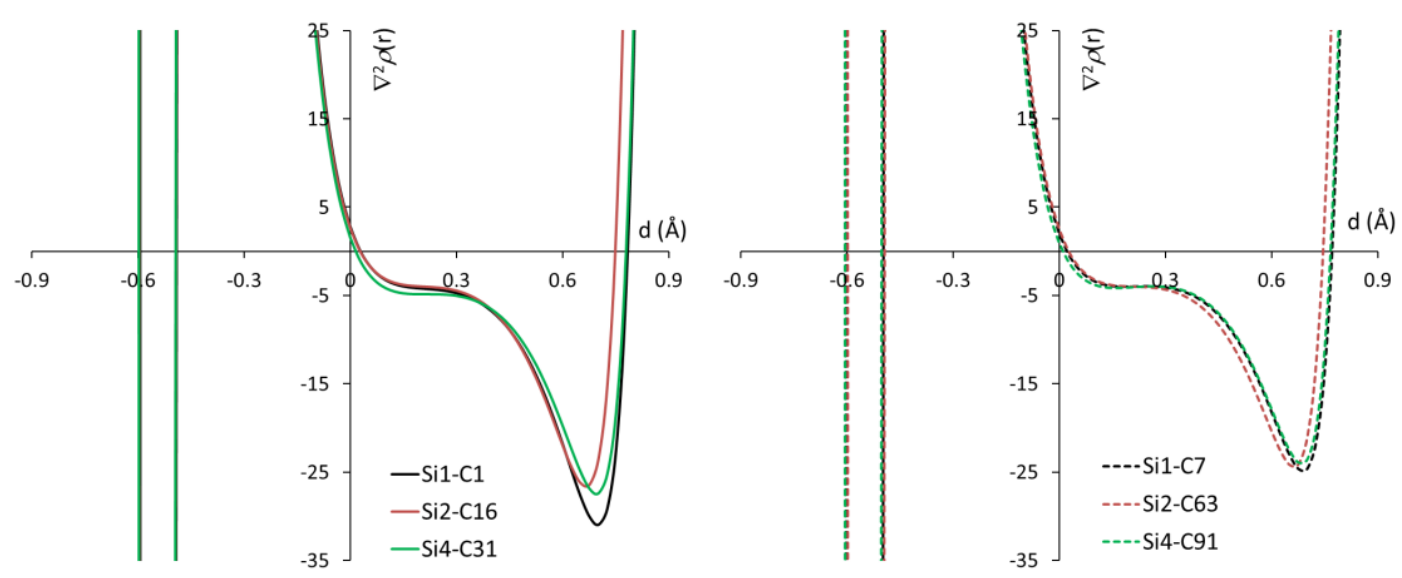

Figure 40 Distribution of $L(\boldsymbol{r})$ along selected $\mathrm{Si}-\mathrm{C}$ bond paths from experimental data (left) and theory (right). The same color indicated equivalent bonds, see chapter 10.5.3 for numbering scheme.

In conclusion, the distribution of $L(\boldsymbol{r})$ along the bond paths documents that all silicon bonds except the Si1-Si2 bond are very similar.

\subsubsection{Static Deformation Density Maps of 2}
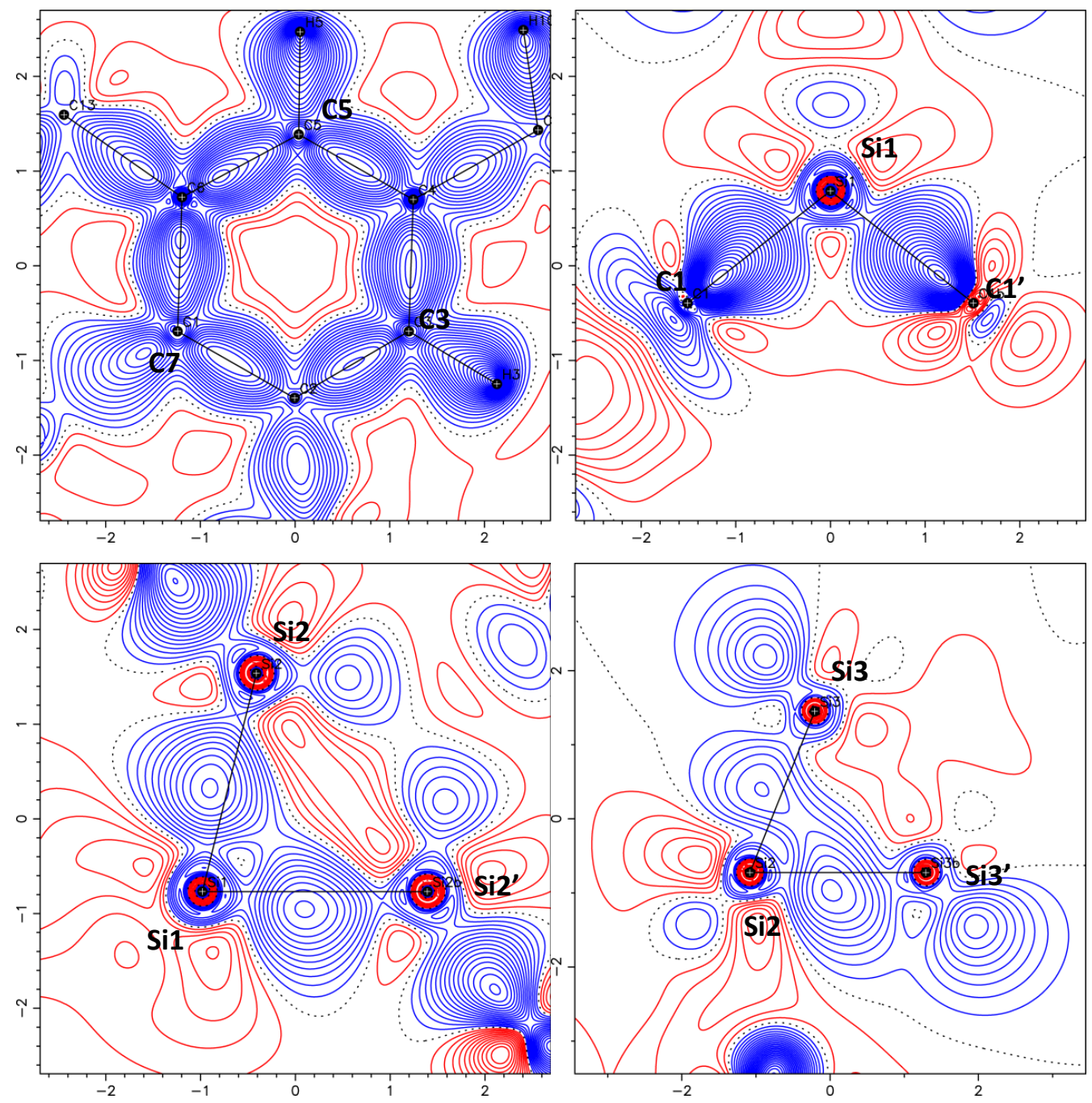


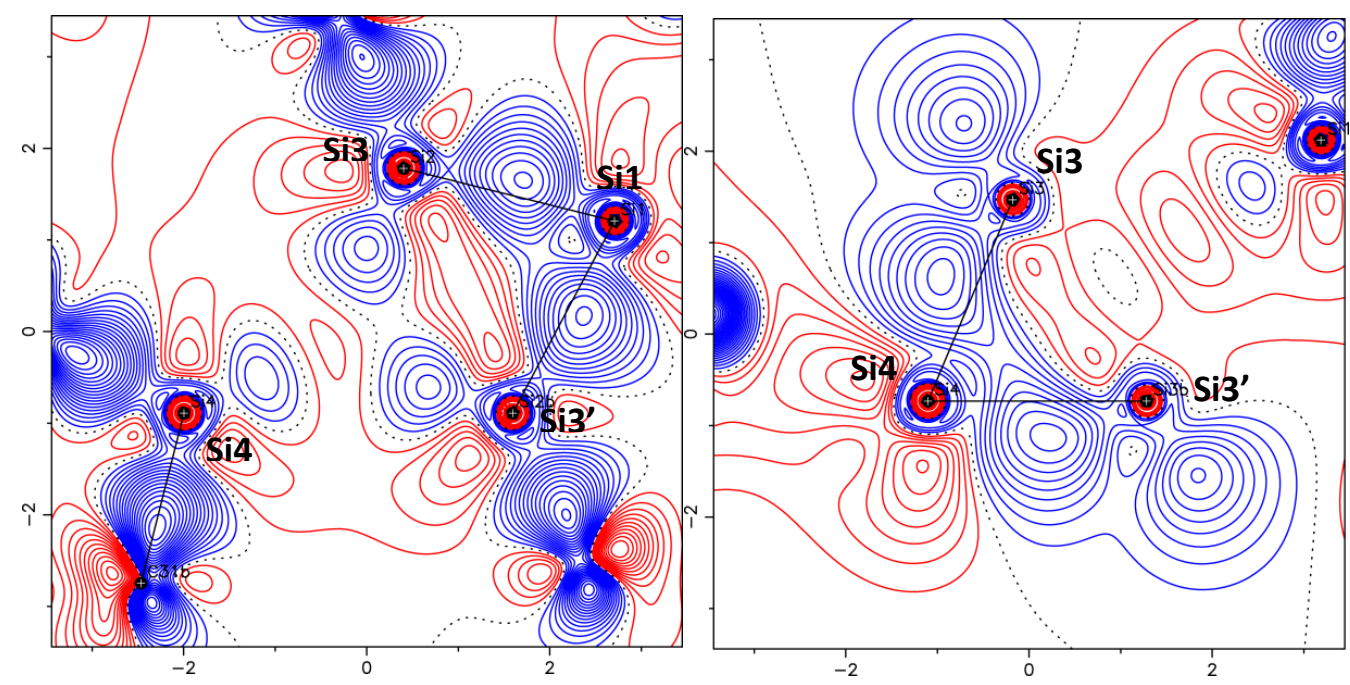

Figure 41 Contour plots of static deformation density of 2 . Contour lines are drawn at $\pm 0.02 \mathrm{e}^{-3}$ level (first plot at $0.04 \mathrm{e}^{-3}$ ).

Depicted in Figure 41 are several plots of the static deformation density in different planes of the molecule 2. All Si-Si bonds show clear indications of bent bonds with the electron density out of the straight line between two atoms. The density at the apical atoms which is directed to the bridgehead atoms is not fully separated. This is an indicator for delocalization of bond density in the cage system of 2 .

\subsubsection{Ellipticity along the Bond Path}
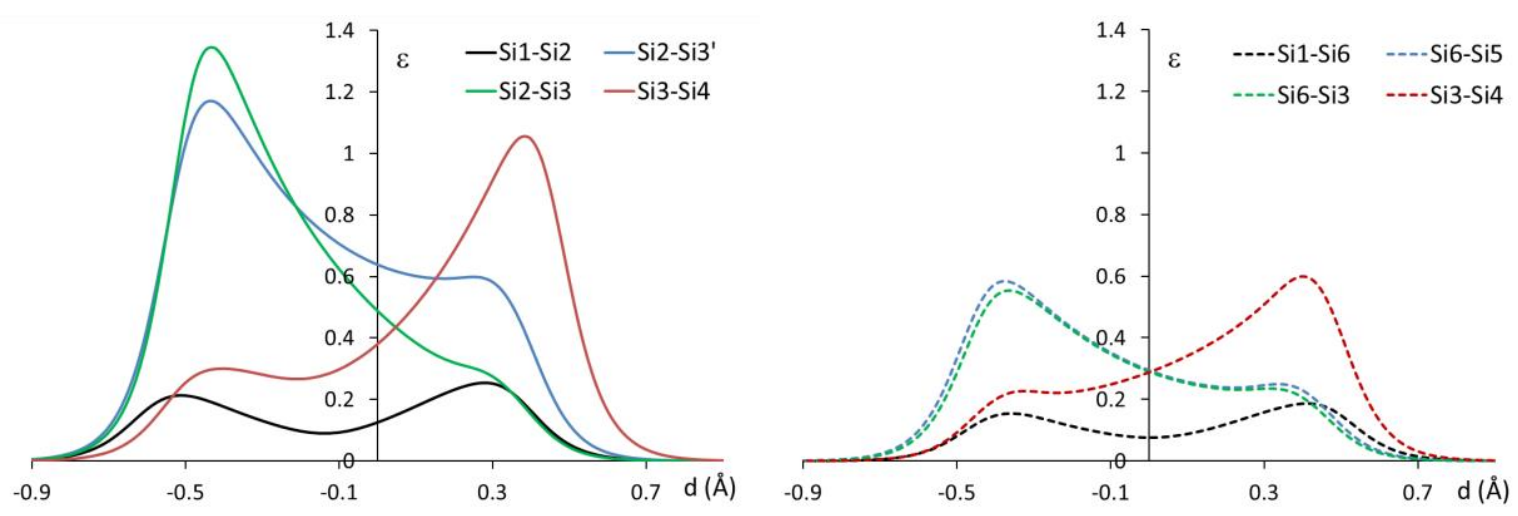

Figure 42 Distribution of $\epsilon$ along the Si-Si bond paths from experimental data (left) and theory (right). The same color indicated equivalent bonds, see chapter 10.5 .3 for numbering scheme.

The shape of a plot of experimentally determined $\epsilon$ values along the Si-Si bond paths Si-Si bonds matches theory remarkably well. However, the absolute value is more distinct in the experiment. According to the distribution of $\epsilon$ the $\mathrm{Si} 1-\mathrm{Si} 2$ bond behaves like a single bond with low $\pi$-density while the other Si-Si bonds have much higher ellipticity. 

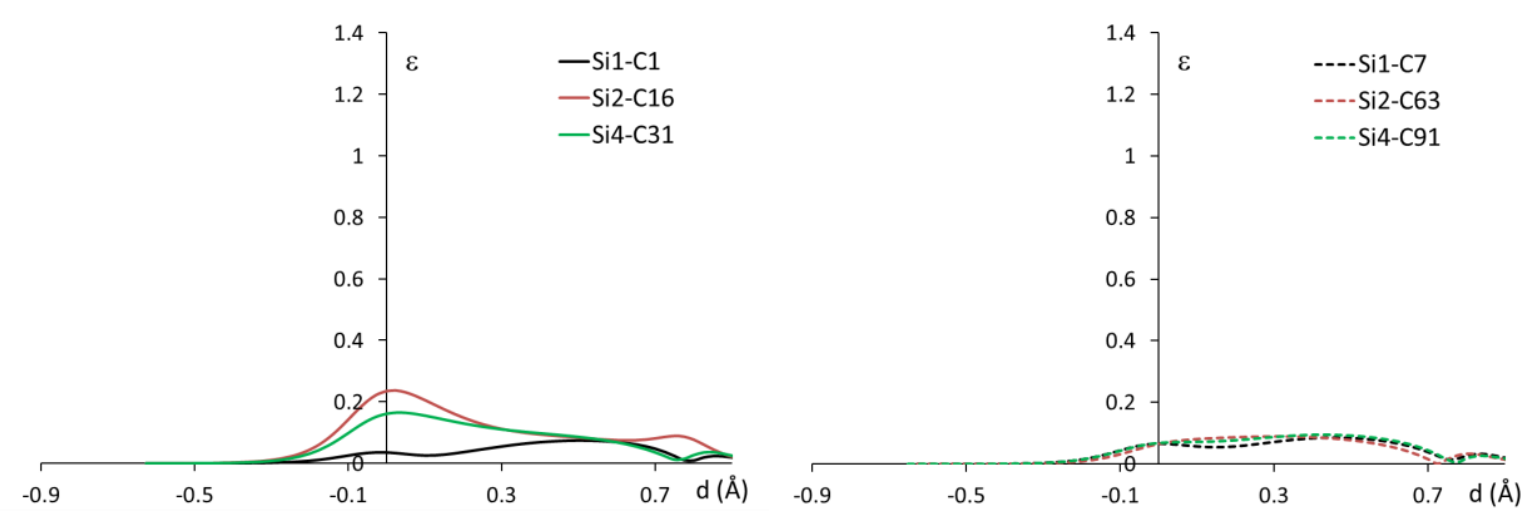

Figure 43 Distribution of $\epsilon$ along the Si-C bond paths from experimental data (left) and theory (right). The same color indicated equivalent bonds, see chapter 10.5 .3 for numbering scheme.

The $\epsilon$ values along the Si-C bond paths in $\mathbf{2}$ have similar distributions as in $\mathbf{1}$ while Si2-C16 and Si4-C31 differ most prominently from the theoretical values (Figure 43). This is consistent with the deviation of $\epsilon$ in the $\mathrm{Si2}-\mathrm{Si} 3$ and the Si3-Si4 bond where the deviation is also most prominent.

\subsubsection{Electrostatic potential}

A comparison of the electrostatic potential mapped on the static electron density from the silicon atoms of $\mathbf{1}$ and $\mathbf{2}$ explains the higher air sensitivity of $\mathbf{2} .^{[106,108]}$ Oxygen as electrophile is attracted by the top of $\mathrm{Si} 3$ in $\mathbf{2}$ (Figure 44 right) where a negative electrostatic potential and accumulation of electron density is observed. The electrostatic potential in 1 (figure 44 left) is everywhere positive around the silicon atoms.

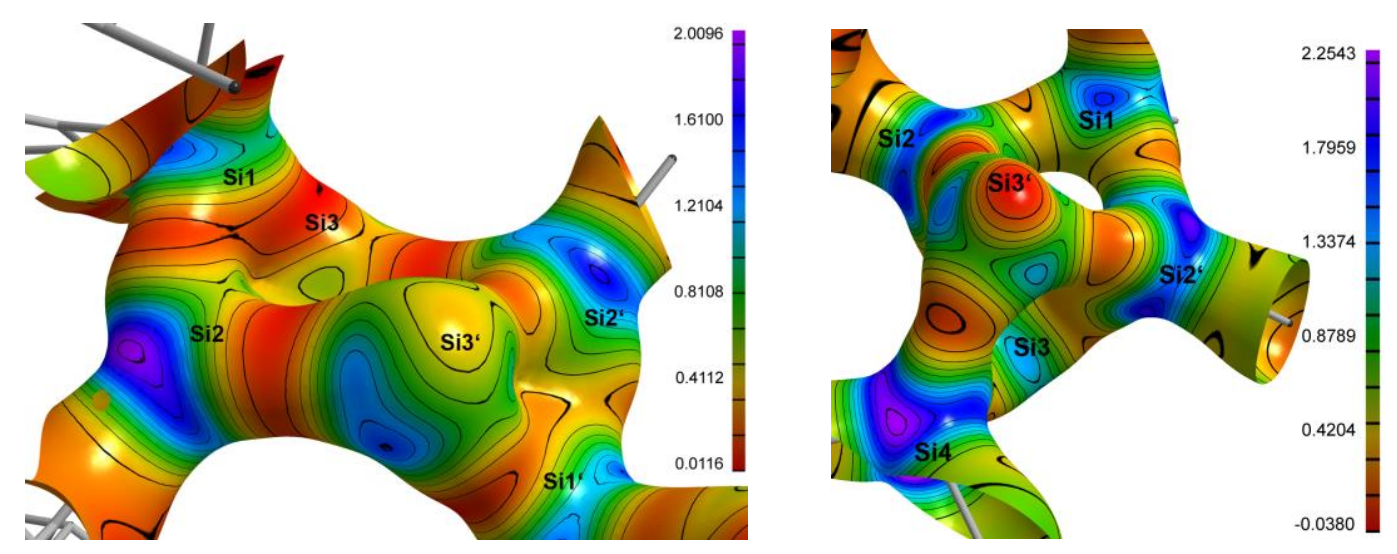

Figure 44 Electrostatic potential in $\AA^{-1}$ mapped on the static electron density at $0.4 \mathrm{e}^{-3}$ level for 1 left and 2 right. 


\subsubsection{Residual Density Distribution}

Table 12 shows the highest peaks and deepest holes in the residual density distribution and their distance to the next atom after multipole refinement.

Table 12 Results from the Fast Fourier Transformation ( $x d f f t$, no cutoff) after final refinement of 2.

\begin{tabular}{lrlllllr}
\hline peak & distance $[\AA]$ & & & $x$ & $y$ & $z$ & \multicolumn{2}{l}{$\begin{array}{l}\text { height } \\
{\left[\AA^{-3}\right]}\end{array}$} \\
\hline \hline $\mathrm{PK}(1)$ & 1.76 & from & $\mathrm{Si}(3)$ & 0.6386 & 0.0562 & 0.7286 & 0.41 \\
$\mathrm{PK}(2)$ & 0.59 & from & $\mathrm{Si}(3)$ & 0.5805 & 0.0776 & 0.7294 & 0.39 \\
$\mathrm{PK}(3)$ & 0.53 & from & $\mathrm{C}(15)$ & 0.2995 & 0.2308 & 0.7997 & 0.34 \\
$\mathrm{PK}(4)$ & 0.41 & from & $\mathrm{Si}(4)$ & 0.5000 & -0.0697 & 0.7500 & 0.33 \\
\hline $\mathrm{HL}(1)$ & 0.55 & from & $\mathrm{C}(1 \mathrm{~A})$ & 0.2595 & 0.6903 & 0.5007 & -0.49 \\
$\mathrm{HL}(2)$ & 0.53 & from & $\mathrm{O}(2 \mathrm{~A})$ & 0.3822 & 0.5317 & 0.5003 & -0.40 \\
$\mathrm{HL}(3)$ & 0.16 & from & $\mathrm{H}(7 \mathrm{~B} 1)$ & 0.3528 & 0.5227 & 0.3981 & -0.28 \\
$\mathrm{HL}(4)$ & 0.44 & from & $\mathrm{C}(24)$ & 0.5626 & 0.0122 & 0.6162 & -0.28 \\
\hline
\end{tabular}

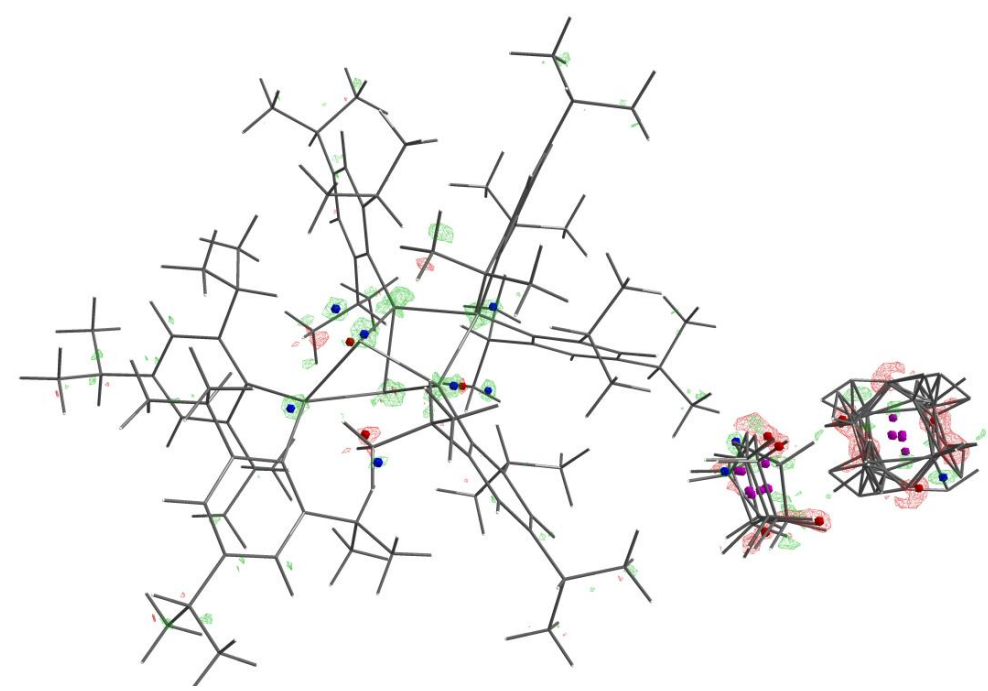

Figure 45 Residual density analysis of 2 after final refinement cycle (all data, contour interval $=0.14 \mathrm{e}^{-3}$ )

The highest peak is located $1.76 \AA$ from Si3 $\left(0.41 \mathrm{e}^{-3}\right)$. The origin of this peak remains unclear. The first idea was a contamination with halogens, but it is too far away from the Si3 to be fluorine and too close to be chlorine or bromine $(\mathrm{d}(\mathrm{Si}-\mathrm{F}) \approx 1.56 \AA \mathrm{A}(\mathrm{Si}-\mathrm{Cl}) \approx 2.07 \AA, \mathrm{d}(\mathrm{Si}-\mathrm{Br}) \approx 2.17 \AA$ ) and also too far away to be $\mathrm{Si}-\mathrm{O}(\sim 1.64 \AA)$. The second highest peaks are located at $\mathrm{C} 15$ of an isopropyl group. However, the residual density was too low to be described by a reasonable disorder model. This residual density and the dynamic disorder of the solvent molecules could not be modeled better because of the lack of restraints in XD2006. 


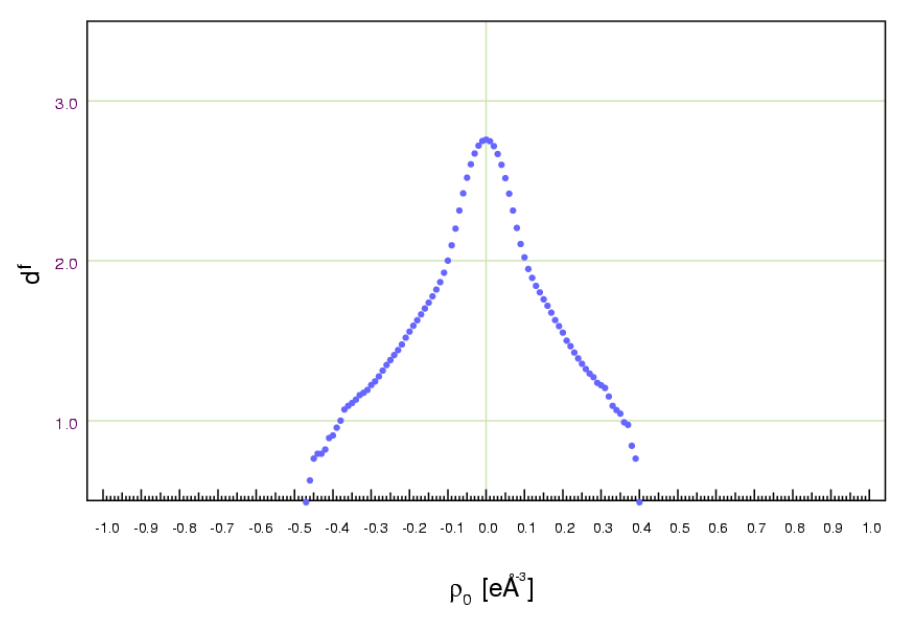

Figure 46 Fractal dimension versus residual density in structure 2.

Inspection of the residual density with jnk2RDA (chapter 3.3.5) results in the distribution of the fractal dimension seen in Figure 46. The deviation from an ideal Gaussian shape originates mainly from the description of the solvent molecules and the isopropyl groups of the Tip ligands which were not ideal. Nonetheless, the multipole refinement delivered very good results due to the high data to parameter ratio of 76 (chapter 5.2.4) as was also the case for $\mathbf{1}$. 


\subsection{Conclusion}

It was possible to obtain convincing electron density models for the challenging structures of 1 and $\mathbf{2}$ by using a combination of a multipole model on the core and an invariom model on the outer disordered entities. It is remarkable how well the model of the central core in both molecules matches the findings obtained from theory. This work would not have been possible without the use of a scattering factor database. The model bias introduced by the database seems to be small enough to get reasonable results for the central core of the molecule. Even the NMR parameters are better explained by the model than the theory. The valence density in the periphery is welldescribed by the invarioms and the charge distribution could be ensured by the monopole refinement. One important factor for the success is that the database approach gives a very large data to parameter ratio.

The experimental charge density investigation shows that the assumption of aromaticity in the ring isomer of hexasilabenzene 1 is valid. Clearly there is a VSCC present in the non-bonding region in the apical position of Si3. Furthermore, the transannular VSCCs of opposite silicon atoms indicate the presence of two transannular bonds. The Si1 atom in structure 1 shows very unusual bonding behavior with their extremely curved bond paths and a broad VSCC pointing in between on the bond density of the central four-membered ring. It behaves more like a three-center bond but also shows the characteristics of a $\sigma$-bond.

An interstitial bond between both $\mathrm{Si}^{0}$ bridgehead atoms in the cage silapropellane conformer 2 could not be found although it is present in the similarly arranged carbon propellane. But carefully inspection of the electron density showed that only slight distortion of the molecule would result in an interstitial bond path. It is very interesting to note that the Bader charges correlate well with the three different oxidation states of the silicon atoms and even respond to the chemically different environment in $\mathbf{2}$. The density distribution in $\mathbf{2}$ clearly shows more aromatic properties than the cyclic compound $\mathbf{1}$. The bridgehead silicon atoms in $\mathbf{2}$ are even slightly negatively charged which is very unusual for silicon atoms.

Further theoretical investigations of the influence of the ligand sphere on the reactivity and stability of both silicon clusters would be interesting. Especially, the influence of the hydrogensilicon bond paths from the Tip ligand to the $\mathrm{Si}^{0}$ atoms would offer exciting research opportunities. However, this would not be possible with experimental data because of the disorder in the ligand sphere.

Additionally, data quality and reliability of modern rotating anode technology in combination with the fast measurement and precise detection abilities of the present APEX II CCD detector was mandatory for this research. The need for high quality data for the refinement of disordered structures must not be underestimated. The upcoming technology of very large CMOS detectors in the combination of shutterless data collection and high brilliant liquid metal X-ray sources will help to get high quality data in the required resolution for charge density investigations.

Further investigations should be made on the automation of the invariom transfer process. It is clear that this method has some limitations, because the automated recognition of molecule topologies is not always unambiguously. The introduction of restraints in the refinement process would bring the method a big step forward. Therefore, different refinement programs should be tested for their suitability. 


\section{Experimental electron density of $\left[\mathrm{Mg}\left\{\left(\mathrm{pz}^{*}\right)_{3} \mathrm{C}\right\}_{2}\right](3)$}

\subsection{Tris(pyrazolyl) Complexes}

Anionic tripodal $\mathrm{C}_{3}$-symmetrical ligands like $\left[\mathrm{MeSi}(\mathrm{NtBu})_{3}\right]^{3-},[164]\left[\mathrm{Sb}(\mathrm{N} t \mathrm{Bu})_{3}\right]^{3-},[165]$ or $\left[\mathrm{S}(\mathrm{NtBu})_{3}\right]^{2-}{ }^{[166-169]}$ were originally designed to coordinate one hemisphere of various metal cations and later turned out to be promising ligands in e.g. enantioselectively catalytic transformations. ${ }^{[170-}$ 172]
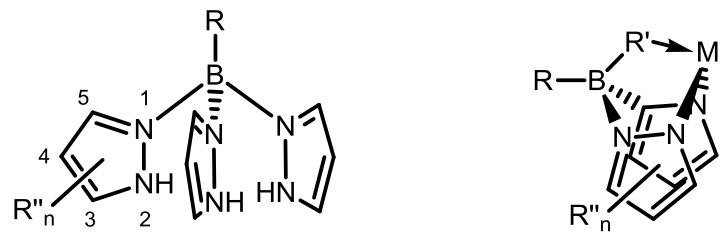

Scheme 27 General structure of trispyrazolylborate anions, $\left[R_{n} B(p z)_{4-n}\right]^{-}$, where $n$ can be 0,1 , or 2 , pz is a pyrazol-1-yl group, and $\mathrm{R}$ can be $\mathrm{H}$, an alkyl, or aryl group.

Hückel's tris(pyrazolyl)methane and Trofimenco's $\left[\mathrm{HB}\left(\mathrm{pz}^{*}\right)_{3}\right]^{-}$scorpionates (Scheme 27) might be regarded as the original templates for this whole class of ligands. ${ }^{[173-178]}$ As pyrazolyl ligands are very suitable ligands in e.g. bio inspired coordination chemistry ${ }^{[179]}$ this original tripodal motif was recently modified by switching the anchor atom to tin, ${ }^{[180-182]}$ germanium ${ }^{[180]}$, silicon ${ }^{[183]}$ or carbon. ${ }^{[184-196]}$ These compounds can serve as site selective Janus-head ligands ${ }^{[197-198]}$ because they provide the $\mathrm{N}$-chelating coordination site and the lone-pair at the anchor atom. Hence, they are interesting ligands to form multimetallic complexes. ${ }^{[199]}$
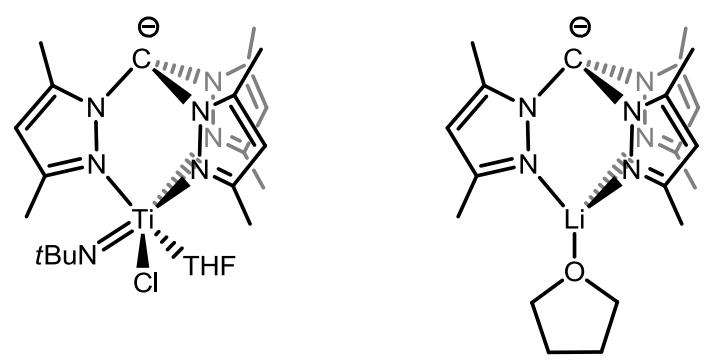

Scheme 28 tris(3,5-dimethylpyrazolyl)methane complexes.

The work presented in this chapter on tris(3,5-dimethylpyrazolyl)methane complexes with $\left[\mathrm{Ti}(\mathrm{NtBu}) \mathrm{Cl}_{2}(\mathrm{py})_{3}\right]$ is based on the work of Mountford et al. (Scheme 28 left). It features a free carbanion with pyramidal geometry which is without any significant intermolecular interactions. ${ }^{[193]}$ Later, Breher et al. made a similar molecule with lithium as anchor (Scheme 28 right). ${ }^{[194]}$ Many different zwitterionic tris-pyrazolylmethane complexes were found afterwards. ${ }^{[193,200-202,196]}$ This chapter is based on the molecule of Breher et al. with two carbanionic ligands complexing a central magnesium atom. 


\subsection{Experimental Details}

The crystals of $\left[\mathrm{Mg}\left\{\left(\mathrm{pz}^{*}\right)_{3} \mathrm{C}\right\}_{2}\right]$ (3) were synthesized and crystallized in the work group of Prof. Breher (Institut für Anorganische Chemie des Karlsruher Institut für Technologie, KIT). The measurement was performed according to chapter 4.2.1 and the data was collected on a Bruker smart APEX II Ultra with a TXS Mo rotating anode, Incoatec mirror optics and an APEXII detector. The dataset was collected at $15 \mathrm{~K}$ with an Oxford Cryosystems Helijet crystal cooling device. To minimize the formation of ice on or near the crystal, the upper half of the diffractometer was enclosed in a transparent plastic cover (Figure 47). This had the effect that the chamber was filled with helium during the measurement and no moisture or oxygen could crystallize anymore. The helium consumption out of the 450 I helium dewar was monitored with four bathroom scales. The accuracy of the weight measurement turned out to be very precise. Especially considering that the weight difference was only $\sim 50$ kilo with a total weight of $\sim 400$ kilo. The helium consumption was $4.17 \mathrm{~kg}$ liquid helium per day or 1.40 I per hour. This led to a total measuring time of 10 days with a 450 I dewar.
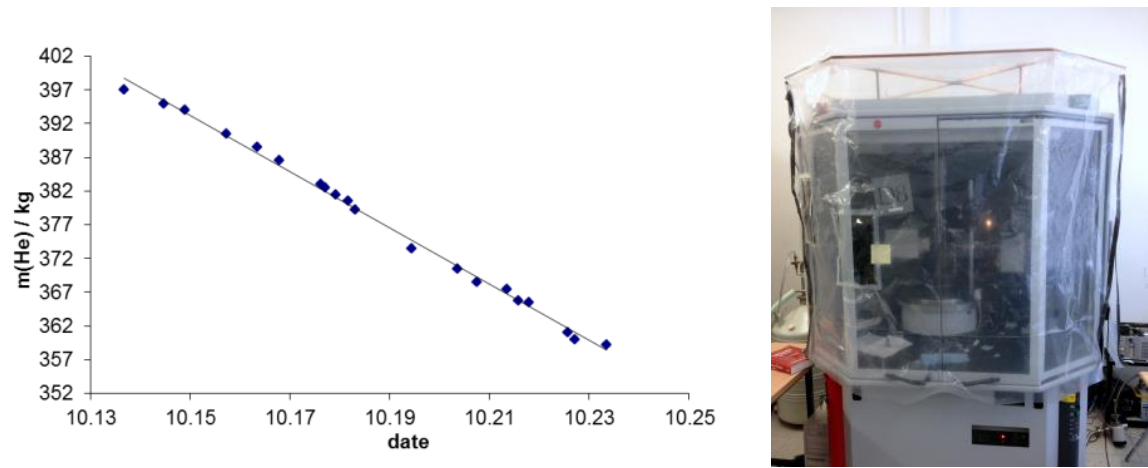

Figure 47 Liquid helium consumption of the Helijet cooling device (left). Plastic cover to enclose the helium during the measurement (right).

3 crystallizes in the space group $R \overline{3}$ with $M g 1$ on the special position $x=1 / 3, y=2 / 3, z=1 / 6$, leading to a symmetrical 6 -fold coordination of the central metal atom with one nitrogen atom of each pyrazolyl group. The asymmetric unit contains just one sixth of the complex due to the high symmetric space group.
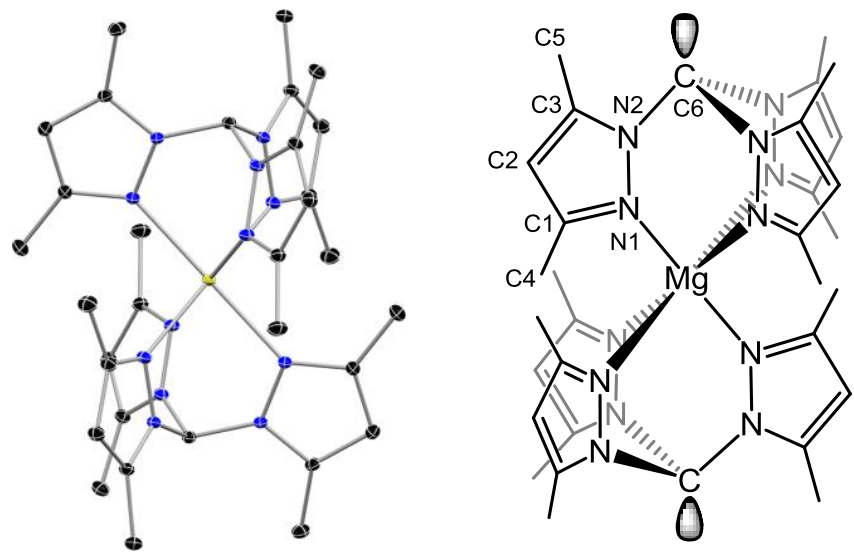

Figure 48 Molecular structure of $\left[\mathrm{Mg}\left\{\left(\mathrm{pz}^{*}\right)_{3} \mathrm{C}\right\}_{2}\right](3)$ with anisotropic displacement parameters depicted at $50 \%$ probability level (left) and Lewis diagram (right). 
The data set was collected in the omega-scan mode with $\Delta \omega=0.3^{\circ}$ at fixed $\varphi$-angles with a detector distance of $5 \mathrm{~cm}$ and an exposure time of $4 \mathrm{~s}$ for the low-order runs with $2 \theta$ at $-33^{\circ}$, $20 \mathrm{~s}$ exposure for the runs with $2 \theta$ at $-45^{\circ}$ and high-order runs with $40 \mathrm{~s}$ exposure time for $2 \theta$ at $-78^{\circ}$. Each run was measured with a sweep of $180^{\circ}$ in $\omega$. The unit cell was determined with the $A P E X I I$ suite ${ }^{[203]}$ and the subsequent data reduction was performed with SAINT V7.68A. ${ }^{[62]}$. Several tests have been made to integrate with a fixed integration box size, but the results turned out to be better with the box size refinement activated. Three different integrations were performed for the three different $2 \theta$ positions. The high-order data was specially treated to correct slightly slipping of the crystal during the measurement. For each high-order run an individual .p4p file was created for which SAINT could determine a different orientation matrix to get a better starting value for the integration. For details about this see chapter 4.2.3. Afterwards, the data was scaled, corrected for absorption and merged with SADABS 2008/2. The absorption correction was done with the empirical method of Blessing ${ }^{[204]}$ because the numerical method with indexed crystal faces gave worse results due to the low absorption of the crystal $\left(\mu=0.1 \mathrm{~mm}^{-1}\right)$.

The starting model for the multipole refinement was obtained as described in chapter 4.4.1. For the methyl groups $\mathrm{C} 4$ with rotational disorder, the procedure was different. The electron density of hydrogen atoms is low so that their position is hard to detect with X-ray data, even without disorder. Consequently, it was not possible to place the hydrogen atoms of a disordered methyl group just by assigning their position from peaks in the difference electron density map.
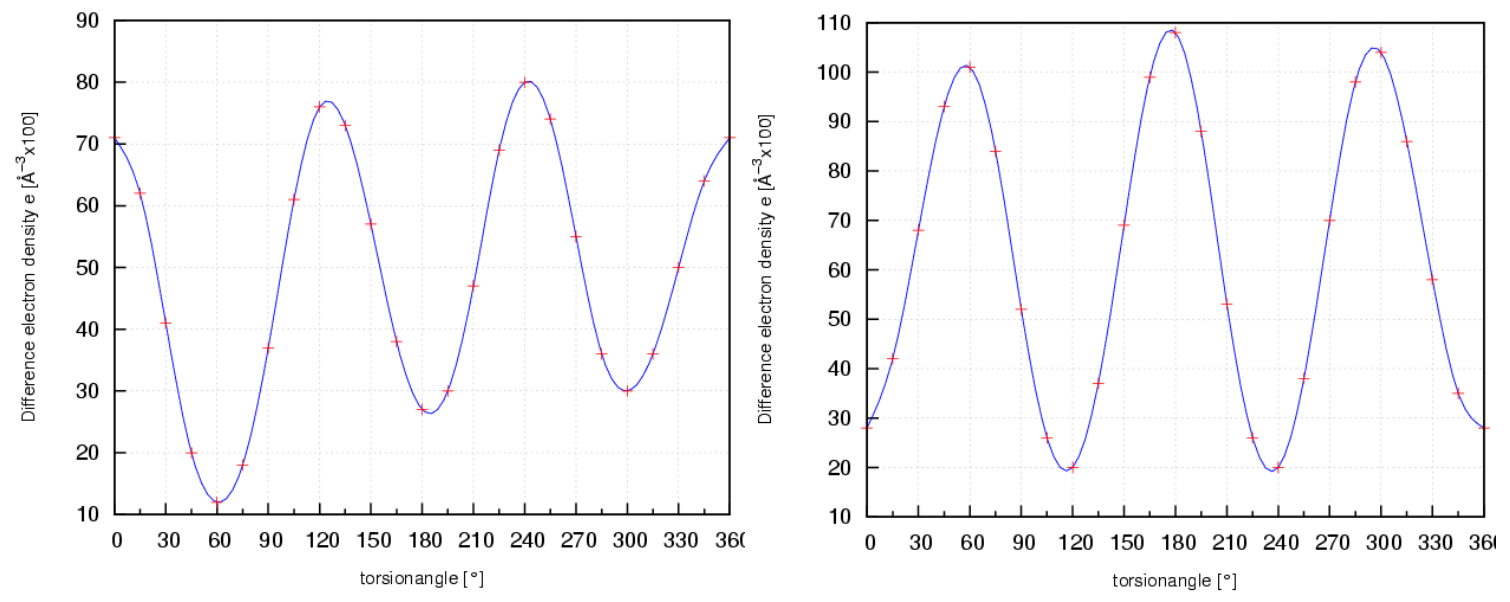

Figure 49 Difference electron density in the cone of the hydrogen atoms around C4 (left) and C5 (right) in 3.

Figure 49 shows the difference electron density given in the .Ist file of SHELXL in the cone of the hydrogen atoms around $\mathrm{C} 4$ and $\mathrm{C} 5$ in compound $\mathbf{3}$. The $\mathrm{C} 5$ atom shows a very symmetrical distribution of the electron density with peaks and minima of almost equal height, whereas the density around $\mathrm{C} 4$ is less symmetric. The lowest values of two minima are higher and the overall heights of the peaks are lower around C4. That clearly shows that the electron density distribution around C4 is not as localized as around C5. Instead the two parts of the methyl group disorder were modeled by AFIX 137 riding model with variable cone angle and free rotation in two parts with SHELXL. This means the cone angle of the $\mathrm{CH}_{3}$ group and the rotation of the two parts were allowed to be refined freely. The occupation factors refined to values of 0.695 and 0.305 , and they were therefore fixed to 0.7 and 0.3 , respectively, during the subsequent refinements. 


\subsection{Multipole Refinement of 3}

The multipole refinement using the atom-centered multipole model of Hansen and Coppens was carried out on $F^{2}$ with the full-matrix-least-squares refinement program $x d l s m$ implemented in the $X D 2006^{[137]}$ program package. Preliminary tested models led to a flat and featureless residual density distribution except a residual density peak at the magnesium atom position. The core and the spherical valence densities were composed of relativistic Dirac-Fock wave functions reported by Su, Coppens and Macchi (SCM bank file). ${ }^{[205-207,143]}$ The radial fit of these functions was optimized by refinement of the expansion-contraction parameters $\kappa$ and $\kappa^{\prime}$. The expansions over the spherical harmonics were truncated at the hexadecapolar level for all hetero atoms and all multipoles $\left(n_{l}=1\right.$ to 4 ) of each atom shared the same $\kappa^{\prime}$-set (keep kappa constraint). The deformation densities of the hydrogen atoms were represented by bond directed dipoles and quadrupoles. To derive adequate parameters for the contraction of the hydrogen atoms, $\kappa$ and $\kappa^{\prime}$ values suggested by Volkov et al. were introduced and kept fixed during the refinement. ${ }^{[17]}$ Moreover, a riding model and distance constraints were applied to the hydrogen atoms during the first steps of the multipole refinement. In the final steps of the refinement the hydrogen atoms (except the disordered group at C4) were refined without distance and thermal motion constraints with a low-order cut-off. After each step the change of the hydrogen parameters was checked for significance.

Several models have been refined and compared, differing in the degree of applied chemical constraints and local non-crystallographic symmetry. A maximum amount of chemical constraints and symmetry restrictions for the multipolar functions stabilized the refinement procedures and reduced correlations. On the other hand the model had to be flexible enough to account for small differences of supposed chemically equivalent atoms. The density parameters were implemented in the refinement routines in a stepwise manner but in the final $18^{\text {th }}$ cycle all parameters but the $\mathrm{K}^{\prime}$ (due to the known effect of large correlations with the other multipole parameters) ${ }^{[17]}$ were refined together using an $I / \sigma(I)$ cut-off of 2 until convergence was reached.

Chemically equivalent or similar atoms were constrained to share the same expansion/contraction parameters, monopole and multipole populations in the beginning of the refinement. In the final refinement stages all constraints for similar atoms were dismissed. Hydrogen atoms with similar chemical environment were modeled with one set of parameters each. The hydrogen atoms $\mathrm{H} 4 \mathrm{~B}$ and $\mathrm{H} 4 \mathrm{C}$ were chemically constrained to $\mathrm{H} 4 \mathrm{~A}$ while $\mathrm{H} 4 \mathrm{E}$ and $\mathrm{H} 4 \mathrm{~F}$ were constrained to H4D. Also $\mathrm{H} 5 \mathrm{~B}$ and $\mathrm{H} 5 \mathrm{C}$ were constrained to $\mathrm{H} 5 \mathrm{~A}$. Due to the diffuse density around $\mathrm{C} 4$ the free refinement of the adjacent hydrogen atoms was not possible. They were treated as a rigid group which was able to move riding on $\mathrm{C} 4$. The final multipole refinement led to a very satisfactory result. The electron density is not biased by it and is well separated from the thermal motion of the non-hydrogen atoms. This was justified by the rigid bond test (DMSDA test) according to Hirshfeld. ${ }^{[208]}$ 
Table 13 Crystallographic information for $\mathbf{3}$ after multipole refinement.

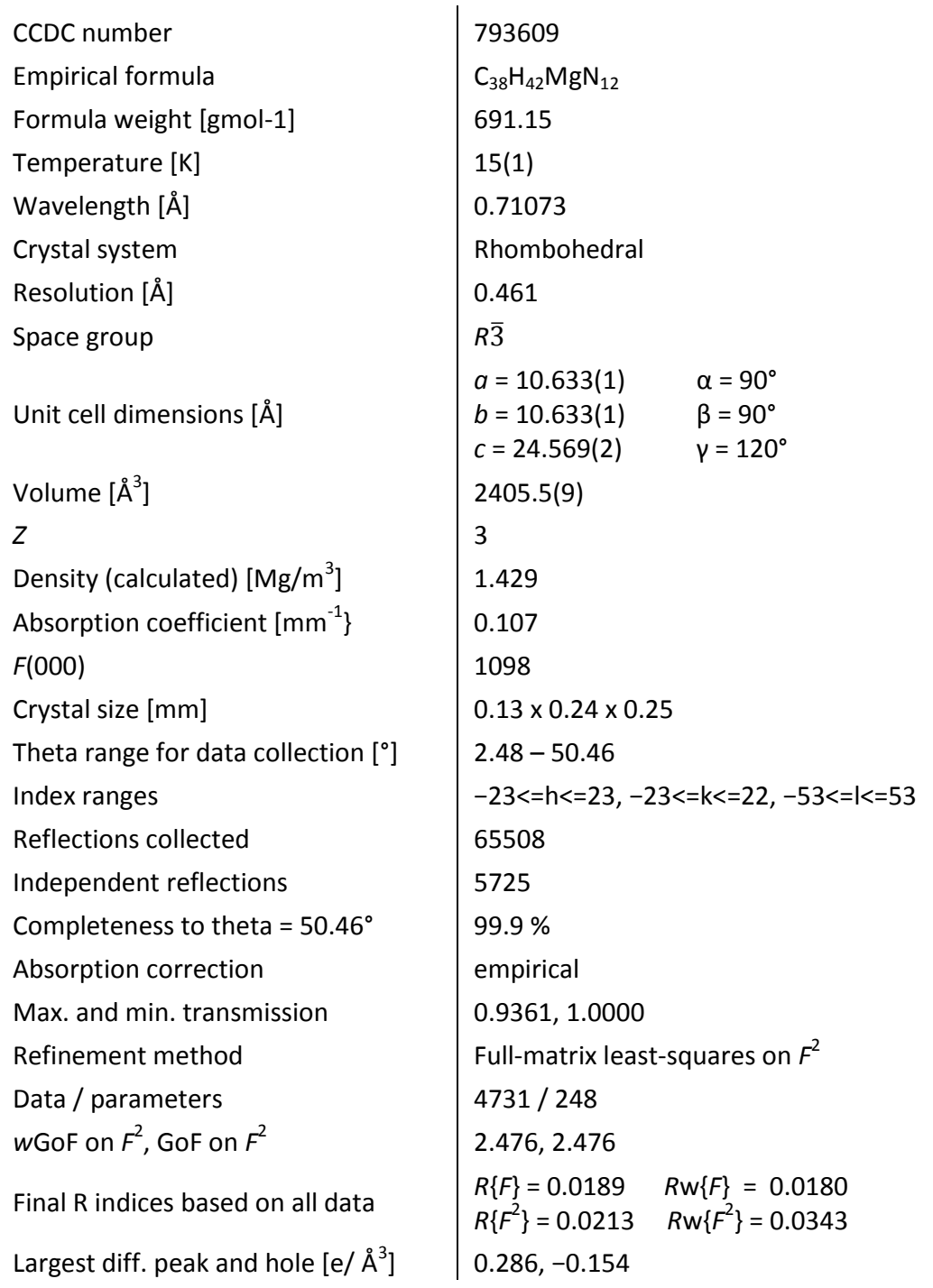

Table 14 Results of the DMSDA test.

\begin{tabular}{ccr} 
(DMSDA) $\left[10^{4} \AA^{2}\right]$ along interatomic bonds \\
\hline N1-N2 & $1.3755 \AA$ & -2 \\
N2-C3 & $1.3532 \AA$ & 2 \\
C1-C2 & $1.4076 \AA$ & 0 \\
C2-C3 & $1.3865 \AA$ & -1 \\
C3-C5 & $1.4869 \AA$ & 6 \\
N1-C1 & $1.3359 \AA$ & 2 \\
N2-C6 & $1.4421 \AA$ & 6 \\
C1-C4 & $1.4918 \AA$ & 3 \\
\hline
\end{tabular}

The central $\mathrm{Mg}^{2+}$ ion is fixed between two $\mathrm{C}\left(\mathrm{pz}^{*}\right)^{3}$ anions. It is coordinated by one nitrogen atom (N1) of each of the six pyrazolyl rings. The carbanionic atom C6 shows tetrahedral environment with the potential lone pair pointing away from the distorted octahedrally coordinated magnesium atom. 


\subsubsection{Residual Density Analysis}

The residual density is in very good approximation flat and featureless, which can be seen by visual inspection of the Fourier maps above or numerically by jnk2RDA developed by Meindl and Henn. ${ }^{[48]}$ The shape of the distribution of the fractal dimension shows a shoulder on low level $\left(<0.3 \mathrm{e}^{-3}\right)$ because of the residual density near the magnesium atom.
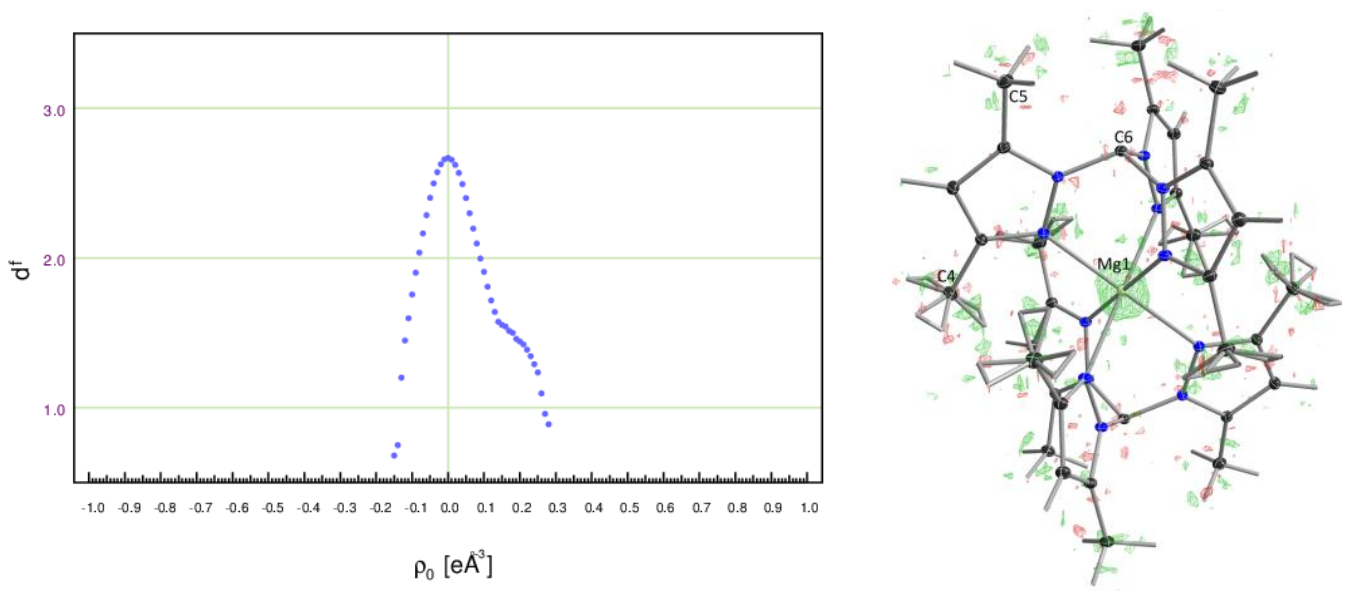

Figure 50 Fractal dimension of the residual density of $\mathbf{3}$ (left) and residual density (right, all data, contour interval $=0.08 \mathrm{e}^{-3}$ )

The origin of the residual density at the Mg1 atom remained unclear. The low residual density in the other areas of the molecule shows that the refinement was nevertheless successful.

\subsubsection{Bond Critical Points and Molecular Graph}

All anticipated critical points could be determined and quantified (Figure 51). Presumably due to the disorder, the bond path between $\mathrm{C} 4$ and $\mathrm{H} 4 \mathrm{~A}-\mathrm{C}$ could not be found. Instead a highly curved bond path between C4 and H4D-F was found. The bond critical points, $(3,-1)$ critical points in $\rho(\boldsymbol{r})$, are displayed as red spheres. The ring critical point, $(3,+1)$ critical points in $\rho(\boldsymbol{r})$, are displayed as yellow spheres. The connection lines between the atoms are the calculated bond paths, lines of maximum density between two atoms. ${ }^{[39]}$ The atoms are represented as blue spheres. 


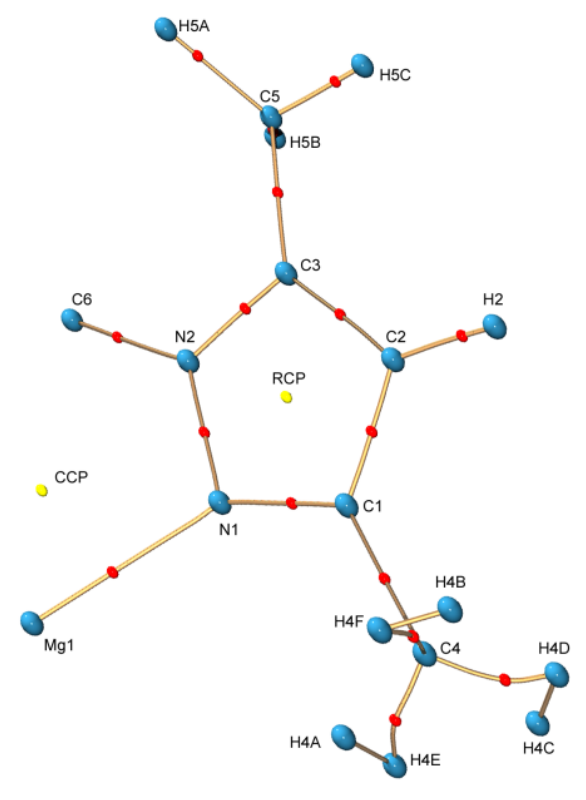

Figure 51 Molecular graph of 3, made up by the bond paths (connection lines), bond critical points (red spheres), ring critical point (RCP, yellow sphere), cage critical point (CCP, yellow sphere), and local extrema of the density (atomic positions, blue spheres).

The bond path between Mg1 and N1 is $2.1973 \AA$ long and the related bond critical point has the properties commonly assigned to closed-shell interactions with low $\left(\rho\left(r_{B C P}\right)=0.21 \mathrm{e}^{-3}\right)$ and a positive $\nabla^{2} \rho\left(r_{B C P}\right)=+3.33 \mathrm{e}^{-5}$. The spatial distribution of the Laplacian field $L(r)$ is positive around the metal ion and reveals a distinct negative value at the nitrogen atom (Figure 52), which can be attributed to the donating nitrogen lone-pair.
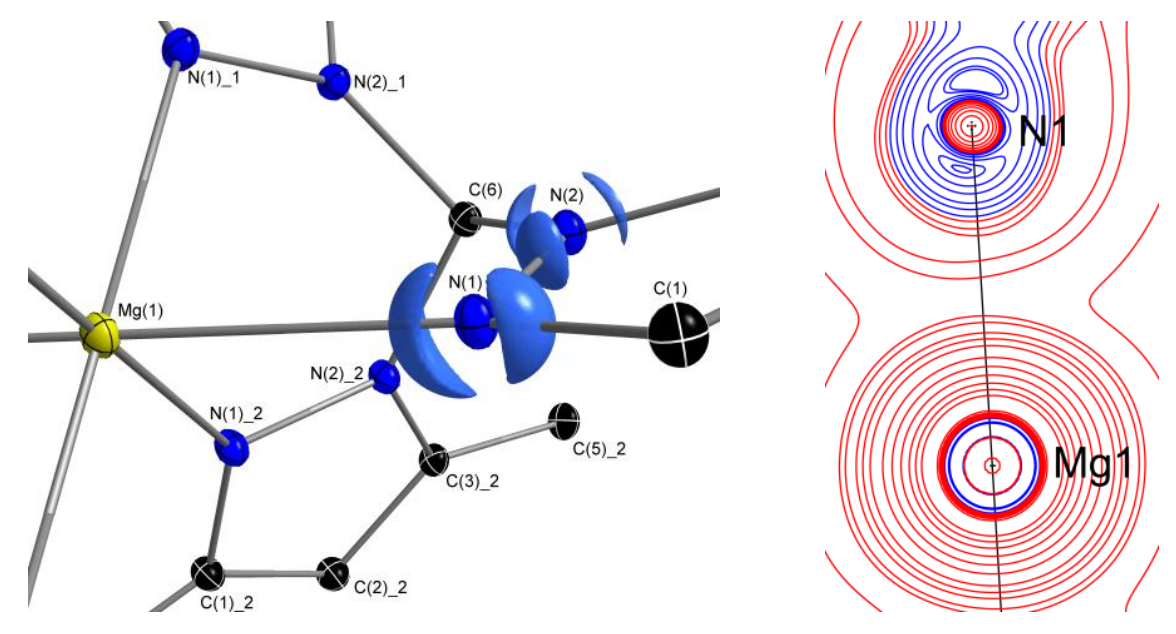

Figure $52 L(\boldsymbol{r})$ around the coordinating nitrogen atom N1.

$L(r)$ along the bond path (Figure 53) is typical for a dative bond in organometallic chemistry: a distinct region of charge concentration in the vicinity of the donating atom (N1) originating from the lone-pair density, which is not far expanded into the bond and a strong depletion of density around the receiving metal ion. ${ }^{[209]}$ In addition the VSCC of N1 to Mg1 does not point directly towards the metal atom which can be clearly seen in Figure 52. 


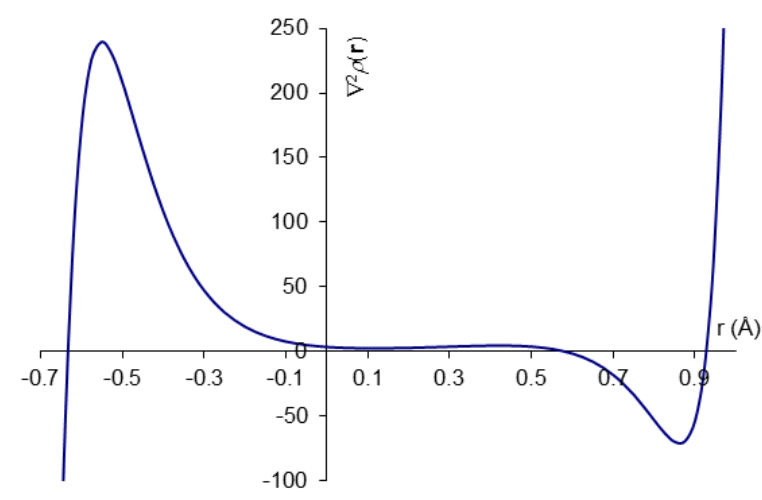

Figure $53 \mathbf{L}(\boldsymbol{r})$ along the Mg1-N1 bond path in $\mathbf{3}$

More than $0.5 \AA$ ahead of the bond critical point, $L(\boldsymbol{r})$ changes the sign and stays positive on a low level, indicating charge depletion over the whole bonding region. $L(\boldsymbol{r})$ rises drastically when reaching the basin of the metal as expected for a cation. The closed-shell nature of the $\mathrm{N}-\mathrm{Mg}$ interaction is displayed in both the distribution of $L(\boldsymbol{r})$ along the bond path as well as the spherically shaped distribution around the cation in space (Figure 52, right). An intriguing feature is the orientation of the pyrazolyl rings to the central magnesium atom. Their planes are rotated along the $\mathrm{C} 6-\mathrm{N} 2$ bond which results in a $0.52 \AA$ shift of the ring mean plane relative to the Magnesium atom.

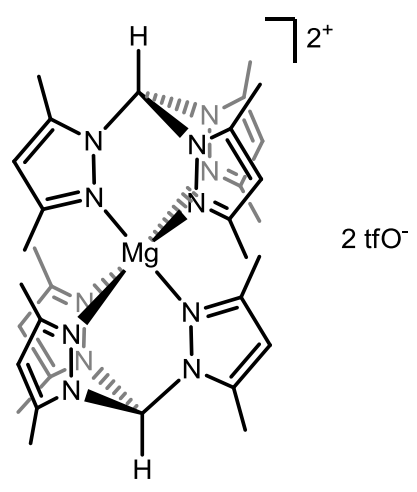

Figure $\mathbf{5 4}$ Molecular structure of $\mathbf{3}$ with protonation at C6.

Compound $\mathbf{3}$ can be easily protonated at $\mathrm{C} 6$ with trifluoromethanesulfonic acid. The resulting $\left[\mathrm{Mg}\left(\mathrm{pz}_{3}{ }_{3} \mathrm{CH}\right)_{2}\right]^{2+} \cdot 2 \mathrm{Otf}^{-}$(Figure 54 ) has some interesting differences compared to 3 . The $\mathrm{C} 6-\mathrm{N} 2$ bonds in $\mathbf{3}$ are slightly elongated, the $\mathrm{CN}_{3}$ bridgehead has a more acute angle, and the rings are rotated about the $\mathrm{C} 6-\mathrm{N} 2$ bond, which results in a more pronounced dislocation of $\mathrm{Mg} 1$ from the plane (0.52 vs. $0.23 \AA$ for the protonated species). ${ }^{[210]}$ However, the $\mathrm{Mg}-\mathrm{C}$ distance is even slightly larger for $\mathbf{3}$ and DFT calculations previously performed on a lithium derivative of $\mathbf{3}$ revealed only small electrostatic interactions between the cation and the anionic carbon atom. ${ }^{[194]}$ Therefore, we assumed the reason might be related to the favorable coupling of the lone-pair at C6 to the $\pi$ density of the pyrazolyl rings (indicated by a quite appreciable ellipticity close to the BCP of C6-N2, Fig. 4). This might cause a propeller-like torsion about the $\mathrm{C} 6-\mathrm{N}$ bonds and the dislocation of the metal from the ring plane. The integrated atomic charge of $\mathrm{N} 1$ within the ring $(0.62 \mathrm{e})$ as well as the bonding criteria for N1-C1 (Table 15) are comparable to those found for other nitrogen heterocycles ${ }^{[209]}$ and do not support any non-typical electronic situation. 
Table 15 Topological parameters of the bond critical points in $\mathbf{3}$.

\begin{tabular}{|c|c|c|c|c|c|c|c|c|c|}
\hline bond & $\begin{array}{l}\rho\left(\mathbf{r}_{\mathrm{BCP}}\right) \\
{\left[\mathrm{e}^{-3}{ }^{-3}\right]}\end{array}$ & $\begin{array}{l}\nabla^{2} \rho\left(\mathbf{r}_{\mathrm{BCP}}\right) \\
{\left[\mathrm{e} \AA^{-5}\right]}\end{array}$ & $\begin{array}{l}d(\mathrm{~A}-\mathrm{B}) \\
{[\AA \AA]}\end{array}$ & $\begin{array}{l}\mathrm{d} 1_{\mathrm{BCP}} \\
{[\AA \AA]}\end{array}$ & $\begin{array}{l}\mathrm{d} 2_{\mathrm{BCP}} \\
{[\AA \AA]}\end{array}$ & $\begin{array}{l}\lambda_{1} \\
{\left[\mathrm{e} \AA^{-5}\right]}\end{array}$ & $\begin{array}{l}\lambda_{2} \\
{\left[\mathrm{e} \AA^{-5}\right]}\end{array}$ & $\begin{array}{l}\lambda_{3} \\
{\left[\mathrm{e}^{-5}\right]}\end{array}$ & $\epsilon\left(\mathbf{r}_{\mathrm{BCP}}\right)$ \\
\hline MG1-N1 & 0.208 & 3.327 & 2.1973 & 0.9402 & 1.2571 & -1.45 & -1.28 & 6.06 & 0.14 \\
\hline $\mathrm{N} 2-\mathrm{C} 6$ & 1.792 & -15.033 & 1.4422 & 0.8629 & 0.5793 & -15.33 & -11.84 & 12.14 & 0.29 \\
\hline N2-N1 & 2.335 & -5.633 & 1.3755 & 0.6997 & 0.6758 & -19.88 & -17.71 & 31.96 & 0.12 \\
\hline $\mathrm{N} 2-\mathrm{C} 3$ & 2.286 & -21.501 & 1.3534 & 0.8006 & 0.5528 & -19.77 & -16.65 & 14.92 & 0.19 \\
\hline C3-C5 & 1.806 & -14.591 & 1.4871 & 0.7612 & 0.7259 & -12.84 & -12.62 & 10.86 & 0.02 \\
\hline C3-C2 & 2.154 & -17.775 & 1.3870 & 0.6959 & 0.6911 & -17.38 & -13.43 & 13.04 & 0.29 \\
\hline $\mathrm{C} 2-\mathrm{C} 1$ & 2.069 & -16.483 & 1.4079 & 0.6932 & 0.7147 & -15.69 & -13.52 & 12.73 & 0.16 \\
\hline N1-C1 & 2.434 & -23.221 & 1.3360 & 0.7688 & 0.5672 & -21.23 & -17.65 & 15.66 & 0.20 \\
\hline $\mathrm{C} 1-\mathrm{C} 4$ & 1.873 & -12.922 & 1.4918 & 0.7389 & 0.7530 & -12.91 & -12.69 & 12.68 & 0.02 \\
\hline $\mathrm{C} 2-\mathrm{H} 2$ & 1.744 & -17.079 & 1.0759 & 0.7373 & 0.3386 & -16.97 & -16.15 & 16.03 & 0.05 \\
\hline $\mathrm{C} 4-\mathrm{H} 4 \mathrm{~A}$ & 2.336 & -15.570 & 1.1849 & 0.6099 & 0.5751 & -18.22 & -8.10 & 10.75 & 1.25 \\
\hline $\mathrm{C} 4-\mathrm{H} 4 \mathrm{~B}$ & 2.334 & -15.553 & 1.1852 & 0.6098 & 0.5754 & -18.22 & -8.09 & 10.76 & 1.25 \\
\hline $\mathrm{C} 4-\mathrm{H} 4 \mathrm{C}$ & 2.333 & -15.555 & 1.1852 & 0.6099 & 0.5753 & -18.23 & -8.09 & 10.76 & 1.25 \\
\hline $\mathrm{C} 4-\mathrm{H} 4 \mathrm{D}$ & 2.333 & -15.555 & 1.1114 & 0.6099 & 0.5015 & -18.23 & -8.09 & 10.76 & 1.25 \\
\hline C4-H4E & 2.336 & -15.570 & 1.1111 & 0.6099 & 0.5013 & -18.22 & -8.10 & 10.75 & 1.25 \\
\hline $\mathrm{C} 4-\mathrm{H} 4 \mathrm{~F}$ & 2.334 & -15.553 & 1.1113 & 0.6098 & 0.5015 & -18.22 & -8.09 & 10.76 & 1.25 \\
\hline$C(5)-H(5 A)$ & 1.715 & -18.877 & 1.0846 & 0.7618 & 0.3228 & -16.65 & -16.02 & 13.79 & 0.04 \\
\hline$C(5)-H(5 B)$ & 1.714 & -18.829 & 1.0845 & 0.7619 & 0.3226 & -16.59 & -16.04 & 13.81 & 0.03 \\
\hline$C(5)-H(5 C)$ & 1.714 & -18.867 & 1.0844 & 0.7615 & 0.3229 & -16.64 & -16.01 & 13.78 & 0.04 \\
\hline
\end{tabular}

$\lambda_{3}$ is the curvature of $\rho(\mathbf{r})$ along the bond path, the ellipticity $\epsilon=\lambda_{2} / \lambda_{1}-1$, dBP the total length of the BP, d1, $2_{B C P}$ the distance of the first and second named atom to the BCP.

The electron density distribution in the $\mathrm{pz}^{*}$-rings is related to the question of the electronic state at $\mathrm{N} 1$. The bonds in the heteroatomic $\mathrm{C}_{3} \mathrm{~N}_{2}$-perimeter are expected either to display $6 \pi$-aromatic character or to exhibit localized double bonds. Consideration of the bond lengths alone would not provide a decisive picture since the differences in the bond path lengths are only marginal (Table 15). The C2-C3 (1.3870 $)$ and N1-C1 (1.3360 $)$ distances are the anticipated double bonds, but they are only $0.02 \AA$ shorter than C1-C2 (1.4079 $)$ ) and N2-C3 (1.3534 $)$ ), respectively. The fact that the bonds are different is much more pronounced in the topological descriptors at the bond critical points and even more in the descriptors along the bond paths:

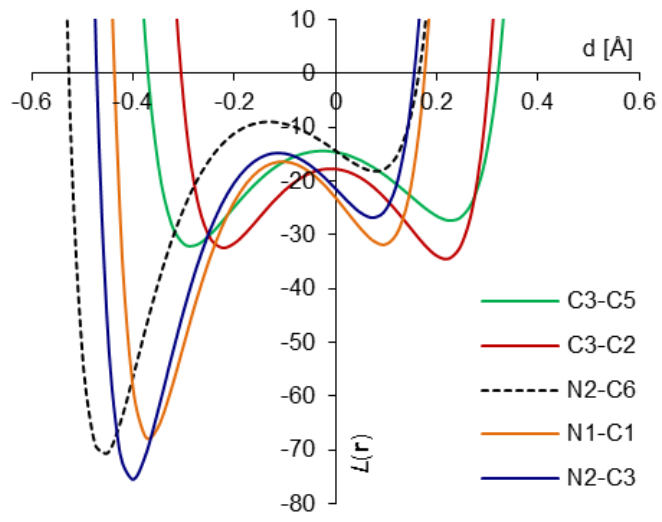

Figure $55 \boldsymbol{L}(\boldsymbol{r})$ distribution along selected bond paths.

The density and $L(\boldsymbol{r})$ at the bond critical points correlates with the lengths of the bond paths. The shorter bonds reveal higher $\rho\left(\boldsymbol{r}_{B C P}\right)$ values and more negative $L(\boldsymbol{r})$ (Table 15). Further insight into the bond properties gives the inspection of the whole bond path. Both $\mathrm{C}-\mathrm{C}$ bonds in the heteroaromatic ring show increased ellipticities over a wide range in the interatomic region. The ellipticity along the bond path of $\mathrm{C2}-\mathrm{C} 3$ is constantly twice as high as that of $\mathrm{C} 1-\mathrm{C} 2$ and very close to the known value from double bonds (Figure 56)..$^{[162,211,209]}$ Less eye-striking, but suiting the 
chemical intuition, are the findings for the $\mathrm{C}-\mathrm{N}$ bonds. $\rho\left(\boldsymbol{r}_{B C P}\right)$ and $\nabla^{2} \rho\left(\boldsymbol{r}_{B C P}\right)$ correlate with the bond lengths differences. $L(\boldsymbol{r})$ along the bond paths is characteristic for shared interactions (Figure 55 ) with a distinct negative vale of $L(\boldsymbol{r})$ over whole bond path. The deep minima in the N2-C6, N1$\mathrm{C} 1$ and N2-C3 originate from polarization the more electronegative nitrogen atoms.

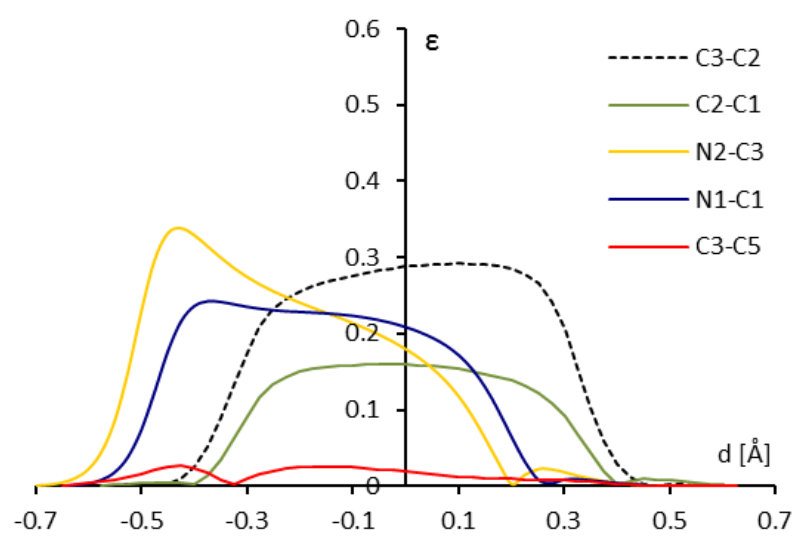

Figure 56 Ellipticity along selected bond paths.

The polarization in the N2-C3 bond is slightly more distinct compared to N1-C1. Here we find less pronounced minima and increased charge concentration in the vicinity of the bond critical point. These findings are supported by the distribution of the ellipticity along the bond path (Figure 55). For N1-C1 it is comparable to $\mathrm{C} 2-\mathrm{C} 3$, but slightly more shifted towards the nitrogen atoms. For N2-C3 we find a comparable absolute value for $\epsilon$, but here it is much more asymmetrically distributed with a well-defined maximum at N2. This feature originates from an overall increased electron density perpendicular to the ring plane at $\mathrm{N} 2$.

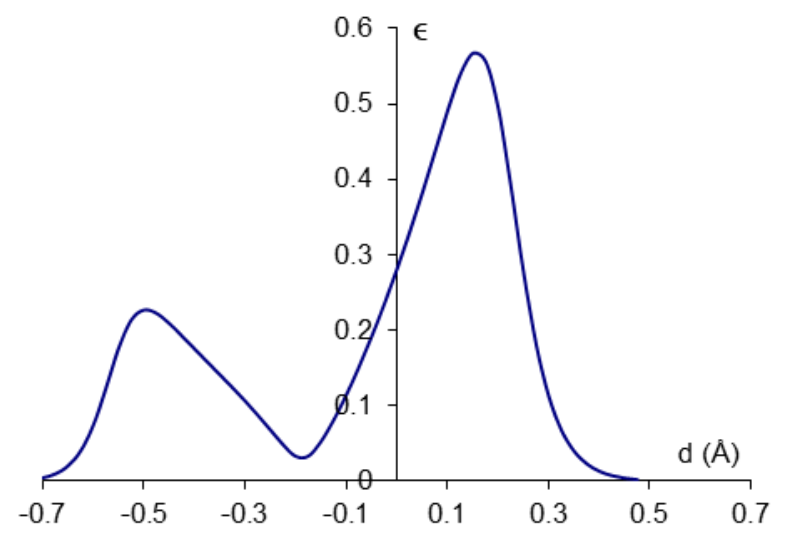

Figure 57 Ellipticity along the bond path bond path from C6 to N2.

The same effect is shown between N2 and $\mathrm{C} 6$ even on higher level. The highest values of $\epsilon$ for all investigated bonds were found close to N2 (0.58, Figure 57). The two peaks in the ellipticity-plot show that the major axis $\left(\lambda_{1}\right)$ of the relatively small ellipticity is found in the region of the carbon atom (left side in Figure 57). Going to N2 it flips into the $\pi$-plane of the nitrogen atom just before the bond critical point is reached. Thus most of the $\pi$-density lies within the basin of the nitrogen atom in the $\mathrm{C}-\mathrm{N}$ bond. 


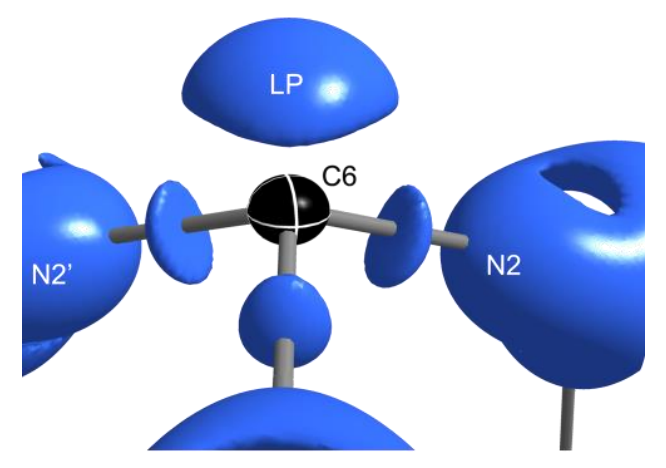

Figure 58 Laplacian distribution at C6 at an isosurface level of $-13.0 \mathrm{e}^{-5}$.

The lone-pair at $\mathrm{C} 6$ is clearly evident from $L(\boldsymbol{r})$. A well-defined lone-pair related charge concentration at a high absolute level $\left[\nabla^{2} \rho(\boldsymbol{r})_{\min }=-30.55 \mathrm{e} \AA^{-5}\right]$ was found in the apical position of C6 (Figure 58). This value is notably higher than other lone-pairs which have been quantified for carbanions, which had VSCCS of $-23 \mathrm{e}^{-5}$ and $-15 \mathrm{e}^{-5}$, respectively. ${ }^{[162,212]}$ The charge density at C6 clearly indicates a carbanion with a stereochemically active lone-pair. Interestingly, the integrated charge at $\mathrm{C} 6$ is +0.64 e and hence unexpectedly positive at first sight. However, it is well known that nitrogen atoms with their high electronegativity tend to contract the atomic basins of the adjacent carbon atoms. ${ }^{[209]}$ The distance from the nucleus position of $\mathrm{C} 6$ to the bond critical point is only $0.58 \AA$, dividing the bond into a ratio of 2:3. The same effect is present at C1 and C3 although at a lower level. Taking into account that $\mathrm{C} 6$ is bonded to three electron withdrawing nitrogen atoms without any other bonding partners, which are able to compensate the polarization (e.g. $\mathrm{CH}$ and $\mathrm{CH}_{3}$ at $\mathrm{C} 3$ ), the charge determined for $\mathrm{C} 6$ is in accordance with the interpretation of $\mathrm{C} 6$ as a carbanion. If there had not been any charge accumulated from the metallation the atom would clearly be considerably more positive than it actually is.

Table 16 integrated atomic charges of 3.

\begin{tabular}{l|r} 
Atom & $\begin{array}{l}\text { integrated } \\
\text { atomic } \\
\text { charge [e] }\end{array}$ \\
\hline Mg1 & +1.840 \\
N1 & -0.618 \\
N2 & -0.733 \\
C1 & +0.334 \\
C2 & -0.196 \\
C3 & +0.353 \\
C4 & -0.427 \\
C5 & -0.516 \\
C6 & +0.636 \\
H2 & +0.201 \\
H5A,B,C & +0.190
\end{tabular}

From the topological features the $\mathrm{C} 6-\mathrm{N} 2$ bond shows no indication of significant bond strengthening due to $\pi$-contributions: the bond is about $0.1 \AA$ longer. The $\rho\left(\boldsymbol{r}_{B C P}\right) 20 \%$, and $\nabla^{2} \rho\left(\boldsymbol{r}_{B C P}\right)$ is about 30\% reduced, compared to the ring $\mathrm{C}-\mathrm{N} \pi$-bonds. Hence, the lone-pair density at $\mathrm{C} 6$ has only, if at all, a minor coupling into the aromatic system of the $\pi$-acceptor $\mathrm{pz}^{*}$ ring system. The origin of the observed dislocated metal remains open. 


\subsection{Conclusion}

Conclusively, the electron density distribution doubtlessly shows the Mg-complex $\mathbf{3}$ to exhibit two carbanions. Two well-defined lone-pairs at the apical positions suitable to act as 2-electron donor for metal coordination were found. ${ }^{[198]}$ The negative charge, even though not straightforwardly reflected by the integrated values, is located at $\mathrm{C} 6$. This could be deduced from the lack of delocalized density from the carbanion towards the heterocycle. The $\mathrm{pz}^{*}$-ring reveals characteristics of localized double bonds, instead of a delocalized $\pi$-system. Increased charge concentration was found at $\mathrm{N} 1$, but no charge transfer to the metal center was observed, leading to the identification of the magnesium atom to be $\mathrm{Mg}^{2+}$ cation and the $\mathrm{N}-\mathrm{Mg}$ bond to be a typical example of a dative lone pair driven bond.

The experiment could show that the combination of a Bruker TXS with an Oxford Cryosystems Helijet is an ideal combination for charge density measurements. The covering of the machine housing to keep the atmosphere around the crystal saturated with helium turned out to be very convenient. No icing at the crystal was observed afterwards which is one of the most common problems with open flow helium cooled measurements. 


\section{Synthesis}

The synthesis of tripodal ligands and their metal complexation was the continuation of the work during my diploma thesis. ${ }^{[13]}$ Different metal complexes of tripodal iminophosphoranes could be synthesized and characterized by single crystal X-ray diffraction. In addition a similar tripodal 1,2,3-triazole containing ligand system was established and characterized by single crystal X-ray diffraction.

\subsection{Tripodal Ligands}

Tripodal ligands where three ligand arms coordinate to a central metal atom are versatile ligands to stabilize metals in a well-defined ligand sphere. The design of the ligand sphere plays a key-role in the design of new systems for asymmetric catalysis. ${ }^{[214-215]}$ For catalysis as such, the ligand-metal system must on the one hand be designed to increase the rate of the desired reaction and on the other hand it must be selective regarding to the stereo chemistry. ${ }^{[216]}$ The selectivity can be improved with distinct ligand symmetry. In chiral catalysts the number of possible diastereomeric intermediates is reduced by the rotational symmetry element. While $C_{2}$ symmetry is widely used in the asymmetric catalysis, ${ }^{[217]}$ there are much less systems with $C_{3}$ or higher symmetry. Burk et al. were the first who introduced a concept for the influence of $C_{3}$ symmetric ligands to stereo selective catalysis (Scheme 29). ${ }^{[218-219]}$ The high selectivity of $C_{2}$ symmetric ligands is generally attributed to the reduced number of possible diastereomeric transition states caused by the two equivalent asymmetric environments along the z-axis (+ and -). ${ }^{[220]}$ In an octahedral catalytic transition state with a chiral $\mathrm{C}_{2}$ symmetric ligand, the substrate binding to the metal has two diastereotopic choices. It can coordinate axial or equatorial (Scheme 29 left).

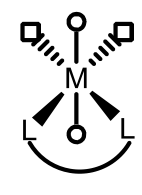

$\mathrm{C}_{2}$

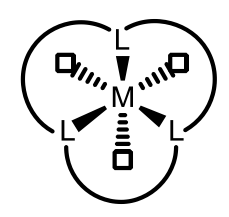

$\mathrm{C}_{3}$

Scheme 29 Ligand model of Burke ( $L$ = Ligand, $M=$ metal).

In an octahedral environment with a $\mathrm{C}_{3}$-symmetric Ligand, all three free coordination sites are equivalent (homotopic). ${ }^{[221]}$ This leads to a reduced number of nonequivalent coordination possibilities and thus to a higher selectivity of the complex. Therefore the stereochemical alternatives would be reduced to the two possible orientations of the prochiral faces with respect to the attacking ligand (Scheme 29 right). 
<smiles>CC(CCc1ccccc1)(Cc1ccccc1)Cc1ccccc1</smiles>

A

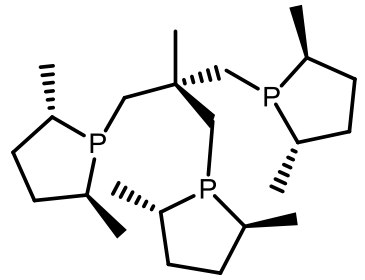

B

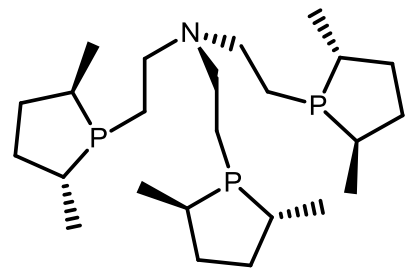

C

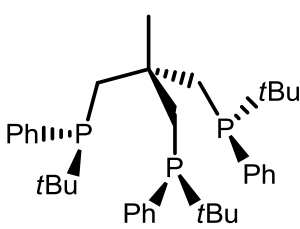

D

Scheme 30 Achiral (A) an chiral (B-D) tripodal phosphane ligands.

Several types of achiral tripodal phosphano ligands were studied especially by Sacconi, ${ }^{[222]}$ Meek ${ }^{[223]}$ Vezani $^{[224]}$ and Bianchini, ${ }^{[225]}$ in the last centuries. They mainly concentrated on triphos (Scheme $30 \mathrm{~A}$ ) and analogous compounds where the central carbon atom is exchanged to a heteroatom like nitrogen or phosphorus. Since these ligands have proven to be versatile ligands in complex chemistry and can form complexes in various oxidation stages it was tried by Gade et al. to build similar chiral derivatives (Scheme 30 B-D). ${ }^{[18]}$

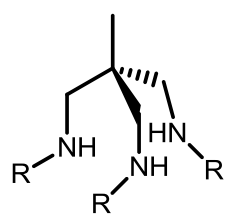

A<smiles>[R]N[SiH2][C@@](C)([SiH3])[SiH](N[R])N[R]</smiles>

B<smiles>[R]N[Si][Si](C)([SiH2]N[R])[SiH2]N[R]</smiles>

C

Scheme 31 Tripodal amino ligands.

A large number of tripodal $\mathrm{C}_{3}$-symmetric amines with a central carbon atom (Scheme $31 \mathrm{~A}$ ) and or a silicon including backbone (Scheme 31 B and C) were synthesized and their metal complexes analyzed in terms of reactivity. ${ }^{[226-230,171]}$ The silicon based ligands can complex bigger metal ions than their carbon based counterparts. With the use of chiral amines, these type of ligands could also be converted to chiral ligands. ${ }^{[231]}$<smiles>[R]N(CC)C12N([R])CCN1CCN2[R]</smiles>

Scheme 32

Tetradentate trisaminoamine ("tren") ligands like in Scheme 32 have been the subject of extensive studies. ${ }^{[232-235]}$ Their specialty is that the central atom can participate in the complexation of the metal atom. The behavior of these ligands is easily changeable through variation of the substituent $R$. Because of the ligand flexibility and the low inversion barrier it is not possible to make chiral ligands through variation of $R$. The generation of chiral tripodal amines can be realized by the functionalization of the ligand backbone. Similar systems are built of $C_{3}$ symmetric trioles und alkoxides. ${ }^{[236-237]}$ 
<smiles>[R]C1COC(C([R])([R])C2=N[C@@H]([R])CO2)=N1</smiles>

A

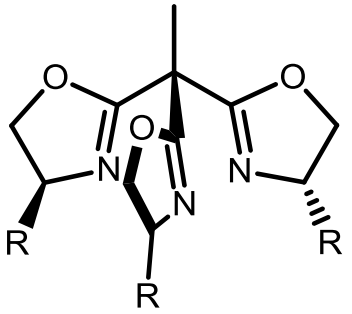

B

Scheme 33 BOX ligand (A) and trisox ligand (B).

A relatively new type of ligand is a polydentate oxazoline. Oxazolines were first published in 1997 as bidentate bis(oxazolinyl)methane ("BOX") ligand, ${ }^{[238]}$ and the tris(oxazolinyl)ethane ligand ("trisox"). ${ }^{[239-240]}$ Both are highly effective stereodirecting ligands. They are mostly used for complexation of late transition metals. The oxazoline ligand stands out in a class of its own, particular in the field of asymmetric catalysis. The oxazoline moiety owes its success to a number of factors: A nitrogen donor atom with in-plane lone-pair and moderate hardness is suitable for a wide range of metals. The synthesis from $\alpha$-amino acids makes derivatives easily accessible und the position of the chiral centre makes them efficient as an asymmetric ligand. The BOX ligand with $\mathrm{CH}_{2}$ and $\mathrm{CHMe}$ bridges can also be used as anionic ligand and is thus analog to the $\beta$-diketiminato ligands

\subsection{Iminophosphoranes}

A recently developed ligand system is built up by tripodal triiminophosphoranes. ${ }^{[241-244]}$ Because of the strong polarization of the $\mathrm{P}-\mathrm{N}$ bond iminophosphoranes are mainly two electron $\sigma$-donors and only weak $\pi$-acceptors. Electron density studies showed that the $\mathrm{P}-\mathrm{N}$ bond is better described as a polarized $\mathrm{P}^{+}-\mathrm{N}^{-}$single bond rather than as a double bond (Scheme 34). ${ }^{[245-246,147]}$ The strong charge at the nitrogen makes it ideal either as a neutral ligand or as an anionic ligand. ${ }^{[247-248,181,249-}$ $255,197,256-258]$
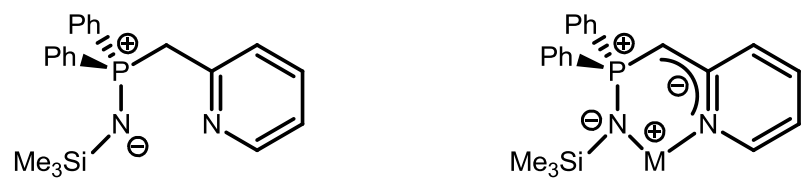

Scheme 34 Electronic distribution in free (left) and metallated iminophosphoranes (right).

The highly charged nitrogen atom in iminophosphoranes makes the formation of metal complexes with low oxidation states difficult but the high donor capability makes them excellent ligands. The potential of iminophosphoranes in coordination chemistry is now well established and recent studies have emphasized their utility as ligands in homogeneous catalysis. ${ }^{[259-264,241,265-}$ $266,243,267]$

The synthesis and crystallization of the iminophosphoranes in chapter 7.6 is the continuation of my previous work. ${ }^{[213]}$ The metal complexes presented herein prove that metals can be coordinated by a tripodal ligand system as shown in Scheme 35 . The ligand system could be further functionalized by the use of different (chiral) phosphines and a different ligand backbone in form of tripodal azides. It would be interesting to try synthesizing an azide with a structure similar to the ligand in Scheme 32 where a central nitrogen atom is able to provide an additional donor possibility. One 
problem with this system is that one precursor of the azide would be a nitrogen lost, also known as "Senfgas", (HN3) which is prohibited to prepare and sell due to the War Weapons Control Act (WWCA).

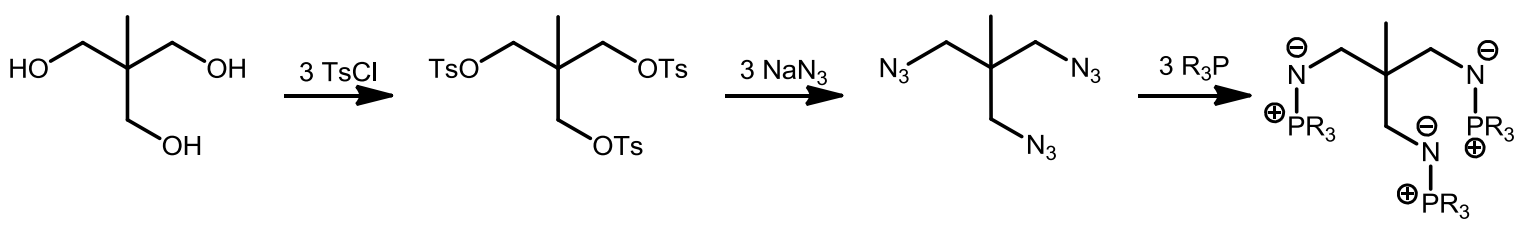

Scheme 35 General synthesis of the tripodal triimonophosphorane ligand systems in chapters 7.6 and 8.

Iminophosphoranes are easily accessible by the reaction of phosphorus(III) compounds with azides in a Staudinger reaction (Scheme 35). The tripodal azide was synthesized from its tosylated alcohol. An alternative reaction to iminophosphoranes is the Kirsanov-Reaction where phosphorus(V) halides react with amines und hydrohalide elimination. ${ }^{[28]}$ However, this reaction is generally not favored because of the tendency to produce more byproducts.

\section{3. $1,2,3$-Triazoles}

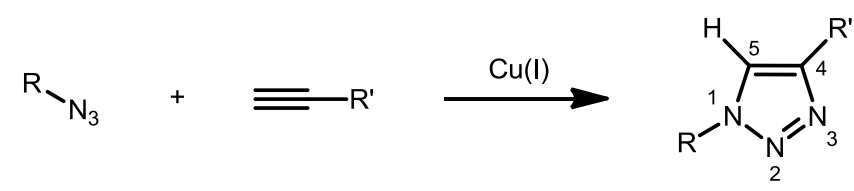

Scheme 36 Copper(I) catalyzed azide-alkyne 1,3-dipolar cycloaddition.

From the tripodal azide in Scheme 35 it is obvious that also azide-alkyne-cycloadditions (AAC) can be used to build a tripodal N-heterocyclic ligand. Meldal ${ }^{[269-270]}$ and Sharpless ${ }^{[271]}$ first described this $\mathrm{Cu}(\mathrm{I})$-catalyzed azide-alkine 1,3-dipolar-cycloaddition (CUAAC) of organic azides with terminal alkynes. This reaction is also known as "click" ${ }^{[272]}$ reaction and it is part of the Huisgen type 1,3dipolar cycloadditions. ${ }^{[273]}$ It is currently receiving considerable attention as a mild, modular method for the generation of functional ligand scaffolds. Furthermore, the 1,4-functionalized 1,2,3triazoles have the potential to act as either neutral $\mathrm{N}$ or carbenoic $\mathrm{C}$ donor ligands. They are already used as mono-- ${ }^{[274-275]} \mathrm{bi}^{\left[{ }^{[276]}\right.}$, tri-, and polydentate ligands with a wide range of metal ions. Because of the tolerance against many functional groups the CUAAC are also used a lot in biochemical systems. ${ }^{[277]}$ Tridentate triazoles are also used for inhibitors against bacterial adhesion, ${ }^{[278]}$ in the cleavage of RNA model phosphate diesters ${ }^{[279]}$ or as anti-inflammatory and anti-cancer drug. ${ }^{[280]}$

A different route to tripodal 1,2,3-triazoles is the reaction of tripodal alkynes with azides. ${ }^{[281]}$ This was not investigated in the current work. The advantage of this method is that it is easier to functionalize the azide scaffold but a disadvantage is that the resulting azide might be more hazardous in terms of explosive decomposition.

Starting from the triazol compound (Scheme 36 ) the tridentate ligand can be easily converted to an $\mathrm{N}$-heterocyclic carbene (NHC). Scheme 37 shows the two most likely binding modes for metals on the ligand system of chapter 7.6 . 

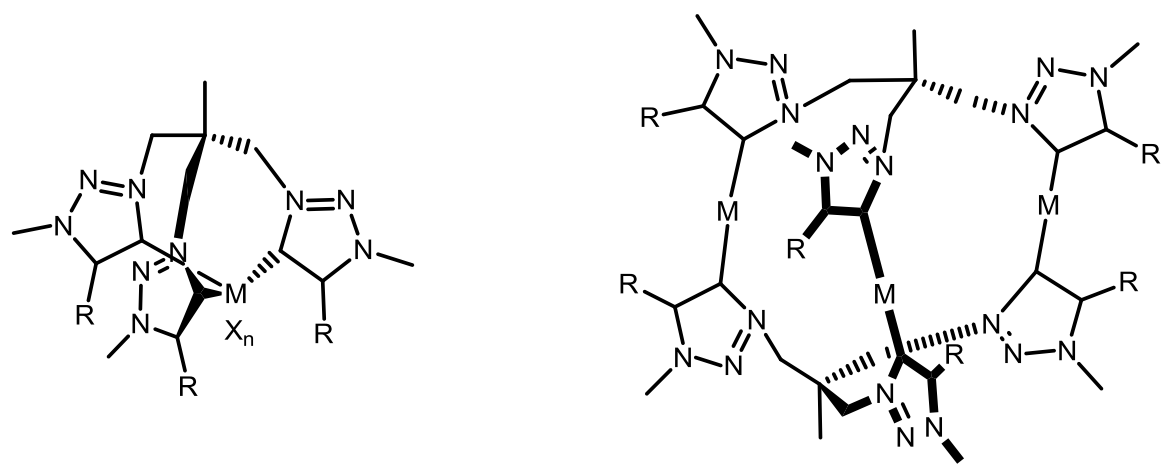

Scheme 37 Possible metal complexes with ligands 15-12 (see chapter 7.6).

Compound 15 in chapter 7.6 can thus be seen as the precursor of the metal complexes shown in Scheme 37.

\subsection{Carbenes}

Carbenes were first mentioned by Wanzlik ${ }^{[282]}$ and Öfele ${ }^{[283]}$ and were pushed forward by Arduengo et al. ${ }^{[284-287]}$ who reported the first stable carbenes. ${ }^{[288]}$

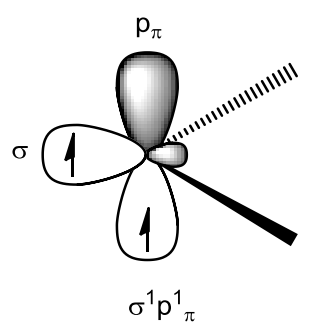

singlett carbene

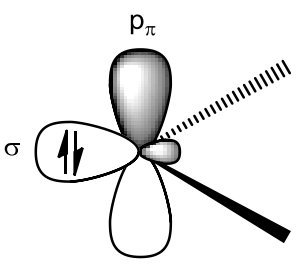

$\sigma 2$

triplett carbene

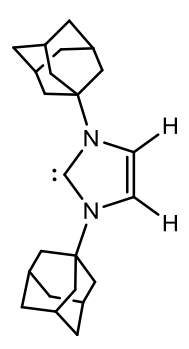

Scheme 38 Electronic configuration at the carbene carbon atom (left). Arduengo carbene (right).

Carbenes are neutral divalent carbon compounds with only six valence electrons. In a formal carbene $-\ddot{C}-$ the arrangement of the two bonds can be linear or angled. The linear geometry implies a sp-hybridization with two non-bonding degenerated orbitals $\left(p_{x}\right.$ and $\left.p_{y}\right)$ at the carbene center. Angular arrangement in cyclic carbenes cancels the degeneration and an $\mathrm{sp}^{2}$-type hybridization is reached. The $p_{y}$ orbital $\left(p_{\pi}\right)$ stays mainly unchanged while the $p_{x}$ orbital $\left(p_{\sigma}\right)$ gains more $s-$ character and is energetically lowered. Two factors play a considerable role for the properties of $\mathrm{N}$ heterocyclic carbenes. The electron withdrawing - 1 effect, on the one hand, stabilizes the nonbonding $\sigma$-orbital by raising its s-character. On the other hand, the energy of the empty $\mathrm{p}_{\pi}$-orbital is lowered by linear combination with the lone pairs of the nitrogen atoms. ${ }^{[289]}$ The impact of NHCs has generally been rationalized by the covalent $\mathrm{M}-\mathrm{C}$ carbene bond and by the strong donor ability. ${ }^{[288]}$ Arduengo-type imidazolylidenes (Scheme 38 ) have been used most widely, presumably because the free carbene is extensively stabilized by heteroatoms adjacent to the carbene, which makes them easy to handle. ${ }^{[284-285]}$

Since the 90s the metal organic carbene complexes attracted an enormous interest in the chemical research. N-heterocyclic carbenes (NHCs) were first intended as simple replacement of phosphano ligands, for example in the Grubbs olefin metathesis system, ${ }^{[290-296]}$ but they are able to outreach them in catalytic activity, range of applications and diversity. Despite the large number of 
stable carbenes, the $\mathrm{N}$-heterocyclic carbenes have gained the biggest distribution in the catalytic chemistry. [289,297-299]<smiles>[R]c1nnn([R])c1[2H]</smiles>

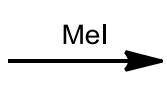<smiles>[R]c1c([2H])[n+](C)nn1[R]</smiles>

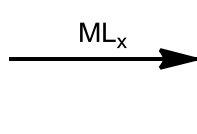<smiles></smiles>

Scheme 39 Metallation of triazolium salts.

\subsection{General Procedures}

All reactions were carried out under Schlenk $k^{[300-301]}$ conditions. Glass ware was heated for several hours at $140{ }^{\circ} \mathrm{C}$, built up hot and cooled to room temperature under vacuum. Air and moisture sensitive compounds were handled under nitrogen atmosphere or in an argon glove-box. All used solvents were freshly distilled and dried with common methods. The metal organic reactants were purchased from the company Rockwood Lithium, all others from the companies Sigma Aldrich and Merck.

Organic azides are potentially-explosive substances. Heat, light and pressure can be initiators for an explosion. The azide 4 is relatively insensitive, it can be heated up to $\sim 200{ }^{\circ} \mathrm{C}$ without decomposition, but the preparation of azides should never be done without blast shield, protective gloves, the quantities should be limited to about $5 \mathrm{~g}$ or less. Sodium azide can form explosive metal azides in contact with metal surfaces e.g. in the laboratory.

\subsection{Synthesis of Tripodal Ligands and their Complexation}

\subsection{1. $\left[\mathrm{NiCl}\left(\mathrm{CH}_{2} \mathrm{NPPhMe}_{2}\right)_{3} \mathrm{CMe}\right]^{+}[\mathrm{Cl}]^{-}(7)$}<smiles>CC(CN=P(C)(C)c1ccccc1)(CN=P(C)(C)c1ccccc1)CN=P(C)(C)c1ccccc1</smiles>

5

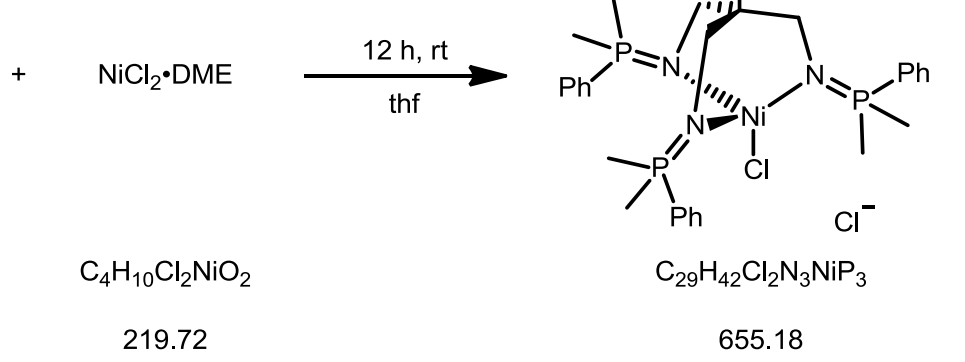

7

$\mathrm{NiCl}_{2} \cdot \operatorname{DME}(2.07 \mathrm{~g}, 9.44 \mathrm{mmol})$ and $5(5.00 \mathrm{~g}, 9.44 \mathrm{mmol})$ were mixed together in THF $(20 \mathrm{~mL})$. The instant deep blue mixture was stirred for $12 \mathrm{~h}$. The little precipitation was filtered off and the filtrate was dried in vacuo. Complex 7 was obtained as blue powder ( $3.86 \mathrm{~g}, 5.85 \mathrm{mmol}, 62 \%)$. For crystallization a small amount (ca. $100 \mathrm{mg}$ ) was mixed with Acetonitrile (ca. $0.3 \mathrm{~mL}$ ) in a dry box. A few drops of toluene were added to the solution until slightly obfuscation appeared. This solution was stored at $-40{ }^{\circ} \mathrm{C}$ over night to get dark-blue crystals suitable for single crystal X-ray diffraction. 


\subsection{2. $\left[\mathrm{SnCl}\left(\mathrm{CH}_{2} \mathrm{NPPhMe}_{2}\right)_{3} \mathrm{CMe}\right]^{+}[\mathrm{Cl}]^{-}(8)$}<smiles>CC(CN=P(C)(C)c1ccccc1)(CN=P(C)(C)c1ccccc1)CN=P(C)(C)c1ccccc1</smiles>

5

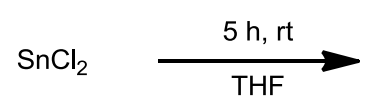

189.62

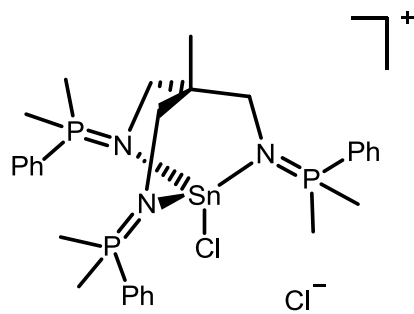

$\mathrm{C}_{29} \mathrm{H}_{42} \mathrm{Cl}_{2} \mathrm{~N}_{3} \mathrm{P}_{3} \mathrm{Sn}$

715.20

To a mixture of $\mathrm{SnCl}_{2}(0.613 \mathrm{~g}, 3.23 \mathrm{mmol})$ and $1(1.70 \mathrm{~g}, 3.23 \mathrm{mmol}) \mathrm{THF}(35 \mathrm{~mL})$ was added at room temperature. A white precipitate began to fall out immediately. The mixture was stirred for another $5 \mathrm{~h}$. The product was filtered off, rinsed with THF $(2 \times 5 \mathrm{~mL})$ and dried in vacuo to obtain 6 as white powder ( $3.68 \mathrm{~g}, 0.514 \mathrm{mmol}, 16 \%$ ). For crystallization a small amount (ca. $200 \mathrm{mg}$ ) was dissolved in hot Acetonitrile. The solution was allowed to cool to room temperature and was stored over night to get colorless crystals.

\subsection{3. $\left[\mathrm{Sn}\left(\mathrm{CH}_{2} \mathrm{NPPh}_{3}\right)_{3} \mathrm{CMe}\right]^{2+} 2[\mathrm{Tos}]^{-}(9)$}<smiles>CCCCCCCCCCCCCCCCN=P(c1ccccc1)(c1ccccc1)c1ccccc1</smiles>

6

$$
\begin{gathered}
+\quad \mathrm{Sn}\left[\mathrm{N}\left(\mathrm{SiMe}_{3}\right)\right]_{2} \\
\mathrm{C}_{6} \mathrm{H}_{18} \mathrm{~N}_{2} \mathrm{Si}_{2} \mathrm{Sn} \\
293.10
\end{gathered}
$$

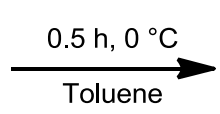

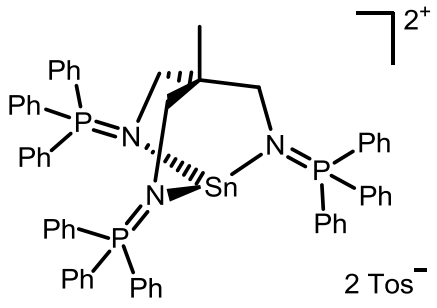

$\mathrm{C}_{59} \mathrm{H}_{54} \mathrm{ClN}_{3} \mathrm{P}_{3} \mathrm{Sn}$ 1052.16 9

$6(0.50 \mathrm{~g}, 0.56 \mathrm{mmol})$ was dissolved in Toluene $(30 \mathrm{~mL})$ and a solution of $\mathrm{Sn}\left[\mathrm{N}\left(\mathrm{SiMe}_{3}\right)\right]_{2}(0.75 \mathrm{~g}$, $1.70 \mathrm{mmol})$ in Toluene $(15 \mathrm{~mL})$ was added at $0{ }^{\circ} \mathrm{C}$ over $30 \mathrm{~min}$. After stirring over $3 \mathrm{~d}$ at r.t. the mixture was stored at $4{ }^{\circ} \mathrm{C}$ over night. Afterwards, a few drops of dioxane were added and the mixture was stored at room temperature to grow colorless crystals. 


\subsection{4. $\left.\left[\mathrm{Sn}\left\{\mathrm{N}\left(\mathrm{SiMe}_{3}\right)_{2} \mathrm{CH}_{2} \mathrm{NP}\left(\mathrm{C}_{6} \mathrm{H}_{4}\right) \mathrm{Ph}_{2}\right)\right\}_{3} \mathrm{CMe}\right](10)$}

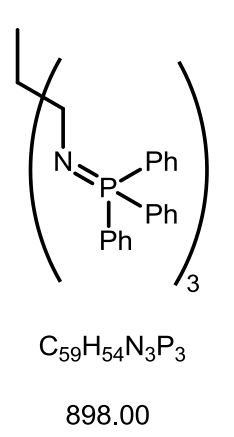

6

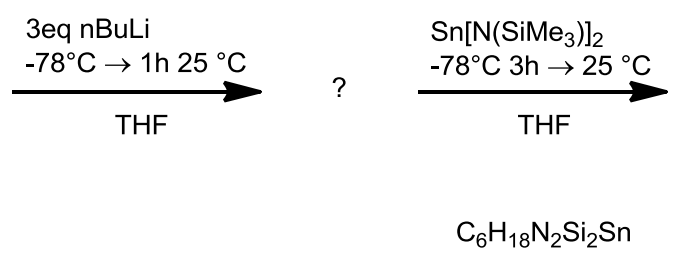

293.10

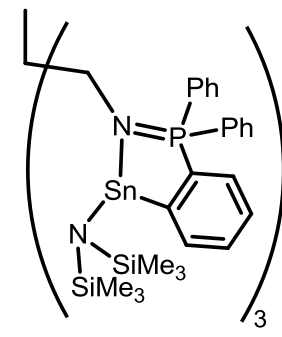

$\mathrm{C}_{59} \mathrm{H}_{54} \mathrm{~N}_{3} \mathrm{P}_{3} \mathrm{Sn}$

1052.16

To a solution of $6(1.00 \mathrm{~g}, 1.11 \mathrm{mmol})$ in THF $(50 \mathrm{~mL}) n B u L i(2.04 \mathrm{~mL}, 3.37 \mathrm{mmol})$ was dropped at $-78^{\circ} \mathrm{C}$ over $1 / 2 \mathrm{~h}$. The solution turned orange. While the mixture was heated over $1 \mathrm{~h}$ to room temperature the solution turned over red to black. The solution was again cooled to $-78{ }^{\circ} \mathrm{C}$ and $\mathrm{Sn}\left[\mathrm{N}\left(\mathrm{SiMe}_{3}\right)\right]_{2}(1.47 \mathrm{~g}, 3.34 \mathrm{mmol})$ solved in toluene $(15 \mathrm{~mL})$ was dropped to the mixture over $1 / 2 \mathrm{~h}$. The color turned to dark green. After warming up to room temperature while stirring overnight, the color turned brown. After storing of the mixture at $4{ }^{\circ} \mathrm{C}$ for two weeks, colorless crystals were grown.

\subsection{5. $\mathrm{MeC}\left\{\mathrm{CH}_{2}\left(\mathrm{~N}_{3} \mathrm{C} 2 \mathrm{H}-4-n \mathrm{Pr}\right)\right\}_{3}(11)$}

To a solution of 1,1,1-tris(azidomethyl)ethane $(3.00 \mathrm{~g}, 15.4 \mathrm{mmol}, 1.00 \mathrm{eq})$ and 1-pentin $(3.14 \mathrm{~g}$, $46.1 \mathrm{mmol}, 3.00 \mathrm{eq})$ in $\mathrm{tBuOH} / \mathrm{H} 2 \mathrm{O}(1: 1,70 \mathrm{ml}), \mathrm{CuSO}_{4} \cdot 5 \mathrm{H}_{2} \mathrm{O}(0.51 \mathrm{~g}, 2.04 \mathrm{mmol}, 4.4 \mathrm{~mol} \%$ of pentin) was added. The suspension changed its color from green over yellow to colorless in ten minutes with a white precipitate. After stirring for $75 \mathrm{~h}$ at room temperature, the two phases were poured in ice water $(90 \mathrm{ml})$ and washed with dichloromethane $(4 \times 30 \mathrm{~mL})$. The solvent of the combined organic phases were removed in vacuo and the crude product was again solved in dichloromethane $(25 \mathrm{ml})$. The solution was extracted with ammonia $(25 \%, 4 \times 20 \mathrm{~mL})$. The ammonia ph $\cdots$ 'vas extracted with dichloromethane $(1 \times 20 \mathrm{ml})$ and the $\cdots \cdots$ nt of the combined organic phé ${ }^{\mathbf{4}}$ las removed in vacuo. The thick mass was again dissc ${ }^{\mathbf{1 1}}$ in THF (15 ml) and diethylether was added until slight precipitation occurred. The flask was cooled in liquid $\mathrm{N}_{2}$ and the precipitate was filtered off $(3.27 \mathrm{~g})$. Pentane was added to the filtrate and the precipitate was filtered off $(1.22 \mathrm{~g})$. The combined white solids were dried in vacuo. 12 was obtained as white powder (4.49 g, $11.2 \mathrm{mmol}, 73 \%$ ).

7.6.6. $\mathrm{MeC}\left\{\mathrm{CH}_{2}\left(\mathrm{~N}_{3} \mathrm{C}_{2} \mathrm{H}-4-\mathrm{Ph}\right)\right\}_{3}(12)$ 


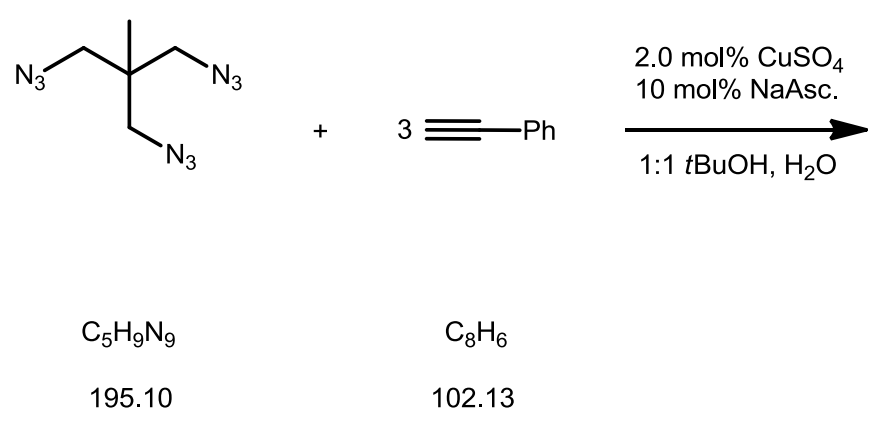<smiles>CC(Cn1cc(-c2ccccc2)nn1)(Cn1cc(-c2ccccc2)nn1)Cn1cc(-c2ccccc2)nn1</smiles>

$\mathrm{C}_{29} \mathrm{H}_{27} \mathrm{~N}_{9}$

501.58

To a suspension of 1,1,1-tris(azidomethyl)ethane $(0.74 \mathrm{~g}, 3.79 \mathrm{mmol}, 1.00 \mathrm{eq})$ and phenyl-ethin (1.22 g, $11.9 \mathrm{mmol}, 3.15 \mathrm{eq})$ in $\mathrm{tBuOH} / \mathrm{H} 2 \mathrm{O}(1: 1,70 \mathrm{ml}), \mathrm{CuSO}_{4} \cdot 5 \mathrm{H}_{2} \mathrm{O}(0.25 \mathrm{~g}, 1.00 \mathrm{mmol}, 2.2 \mathrm{~mol} \%)$ and sodiumascorbate $\left(0.15 \mathrm{~g}, 0.606 \mathrm{mmol}\right.$ in $\left.3 \mathrm{ml} \mathrm{H} \mathrm{H}_{2} \mathrm{O}\right)$ was added. The suspension changed its color from yellow over dark-orange to colorless in ten minutes with a white precipitate. After stirring for $32 \mathrm{~h}$ at room temperature, the two phases were poured in ice water $(90 \mathrm{ml})$ and filtrated. The solvent of the combined organic phases were removed in vacuo and the crude product was again solved in dichloromethane $(25 \mathrm{ml})$. The crude product was washed with water $(2 \times$ $20 \mathrm{ml}$ ) and recrystallized from acetonitrile and 12 was retrieved as white powder $(1.42 \mathrm{~g}$, $0.28 \mathrm{mmol}, 75 \%)$. Crystals for single crystal diffraction were grown through evaporation of a solution in acetonitrile at room temperature.

\subsection{7. $\mathrm{MeC}\left\{\mathrm{CH}_{2}\left(\mathrm{~N}_{3} \mathrm{C}_{2} \mathrm{H}-4-\mathrm{C}_{3} \mathrm{H}_{4}\right)\right\}_{3}(13)$}<smiles>CC(CN)(CN)CN</smiles>

$\mathrm{C}_{5} \mathrm{H}_{9} \mathrm{~N}_{9}$

195.10<smiles>CC(Cn1cc(C2CC2)nn1)(Cn1cc(C2CC2)nn1)Cn1cc(C2CC2)nn1</smiles>

$\mathrm{C}_{20} \mathrm{H}_{27} \mathrm{~N}_{9}$

393.49

To a suspension of $1(1.48 \mathrm{~g}, 0.76 \mathrm{mmol})$ and cyclopropylethyne $(1.49 \mathrm{~g}, 2.25 \mathrm{mmol})$ in ${ }^{t} \mathrm{BuOH} / \mathrm{H}_{2} \mathrm{O}$ mixture $(36 \mathrm{~mL}, 1: 1)$ were added Sodiumascorbate $(0.46 \mathrm{~g}, 0.232 \mathrm{mmol}$, solved in $3 \mathrm{~mL}$ $\left.\mathrm{H}_{2} \mathrm{O}\right)$ and $\mathrm{CuSO}_{4} \cdot 5 \mathrm{H}_{2} \mathrm{O}\left(0.12 \mathrm{~g}, 0.048 \mathrm{mmol}\right.$, solved in $\left.1 \mathrm{~mL} \mathrm{H} \mathrm{H}_{2} \mathrm{O}\right)$. The mixture was stirred for $24 \mathrm{~h}$ at room temperature. The two phases were separated and the aqueous phase was extracted with $\mathrm{Et}_{2} \mathrm{O}(2 \times 30 \mathrm{~mL})$. The combined organic phases were washed with brine $(30 \mathrm{~mL})$ and a saturated aqueous solution of $\mathrm{NaHCO}_{3}(30 \mathrm{~mL})$. Finally the solvent was removed in vacuo and the Product was obtained as white powder (2.27 g, $5.77 \mathrm{mmol}, 76 \%$ ).

7.6.8. $\mathrm{MeC}\left\{\mathrm{CH}_{2}\left(\mathrm{~N}_{3} \mathrm{C} 2 \mathrm{H}-4-\mathrm{SiMe}_{3}\right)\right\}_{3}(14)$ 
<smiles>CC(CN)(CN)CN</smiles>

$\mathrm{C}_{5} \mathrm{H}_{9} \mathrm{~N}_{9}$

195.10
$2 \mathrm{~mol}_{2} \mathrm{CuSO}_{4}$

$\underset{1: 1 \mathrm{tBuOH}, \mathrm{H}_{2} \mathrm{O}}{10 \mathrm{~mol} \% \mathrm{NaAsc} \text {. }}$

$\mathrm{C}_{5} \mathrm{H}_{10} \mathrm{Si}$

98.22<smiles>CC(Cn1cc(S(C)(=O)=O)nn1)(Cn1cc(S(C)(=O)=O)nn1)Cn1cc(S(C)(=O)=O)nn1</smiles>

$\mathrm{C}_{20} \mathrm{H}_{39} \mathrm{~N}_{9} \mathrm{Si}_{3}$

489.84

4

14

To a suspension of $1(1.48 \mathrm{~g}, 0.76 \mathrm{mmol})$ and trimethylsilylethylene $(2.21 \mathrm{~g}, 2.25 \mathrm{mmol})$ in $t \mathrm{BuOH} / \mathrm{H}_{2} \mathrm{O}$ mixture $(36 \mathrm{~mL}, 1: 1)$ were added sodiumascorbate $(0.46 \mathrm{~g}, 0.232 \mathrm{mmol}$, solved in $3 \mathrm{~mL}$ $\mathrm{H}_{2} \mathrm{O}$ ) and $\mathrm{CuSO}_{4} \cdot 5 \mathrm{H}_{2} \mathrm{O}\left(0.12 \mathrm{~g}, 0.048 \mathrm{mmol}\right.$, solved in $\left.1 \mathrm{~mL} \mathrm{H} \mathrm{H}_{2} \mathrm{O}\right)$. The mixture was stirred for $24 \mathrm{~h}$ at room temperature. The two phases were separated and the aqueous phase was extracted with $\mathrm{Et}_{2} \mathrm{O}(2 \times 30 \mathrm{~mL})$. The combined organic phases were washed with brine $(30 \mathrm{~mL})$ and a saturated aqueous solution of $\mathrm{NaHCO}_{3}(30 \mathrm{~mL})$. Finally the solvent was removed in vacuo and the Product was obtained as yellow paste $(2.27 \mathrm{~g}, 5.45 \mathrm{mmol}, 72 \%)$.

\subsection{9. $\left[\mathrm{MeC}\left\{\mathrm{CH}_{2}\left(\mathrm{~N}_{3} \mathrm{C}_{2} \mathrm{H}-4-n \mathrm{Pr}\right)\right\}_{3}\right]^{3+} 3[1]^{-}$(15)}<smiles>CCCc1cn(CC(C)(Cn2cc(CC)nn2)Cn2cc(CCC)nn2)nn1</smiles>

12

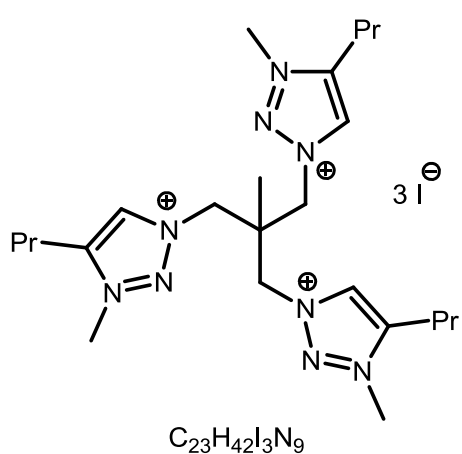

825.35

15

Methyliodide $(4.70 \mathrm{ml}, 75.0 \mathrm{mmol}, 30 \mathrm{eq})$ was dropped slowly to a clear solution of $12(1.00 \mathrm{~g}$, $2.50 \mathrm{mmol}, 1.00 \mathrm{eq})$ in acetonitrile $(20 \mathrm{ml})$. The yellow solution was stirred under reflux for $34 \mathrm{~h}$. The solvent was removed in vacuo and the product was obtained as yellow powder $(1.10 \mathrm{~g}, \mathrm{l})$. Crystals for single crystal diffraction were grown through evaporation of a solution in dichloromethane at room temperature. 


\section{Structures of Self-Synthesized Compounds}

\subsubsection{Unpublished Structures}
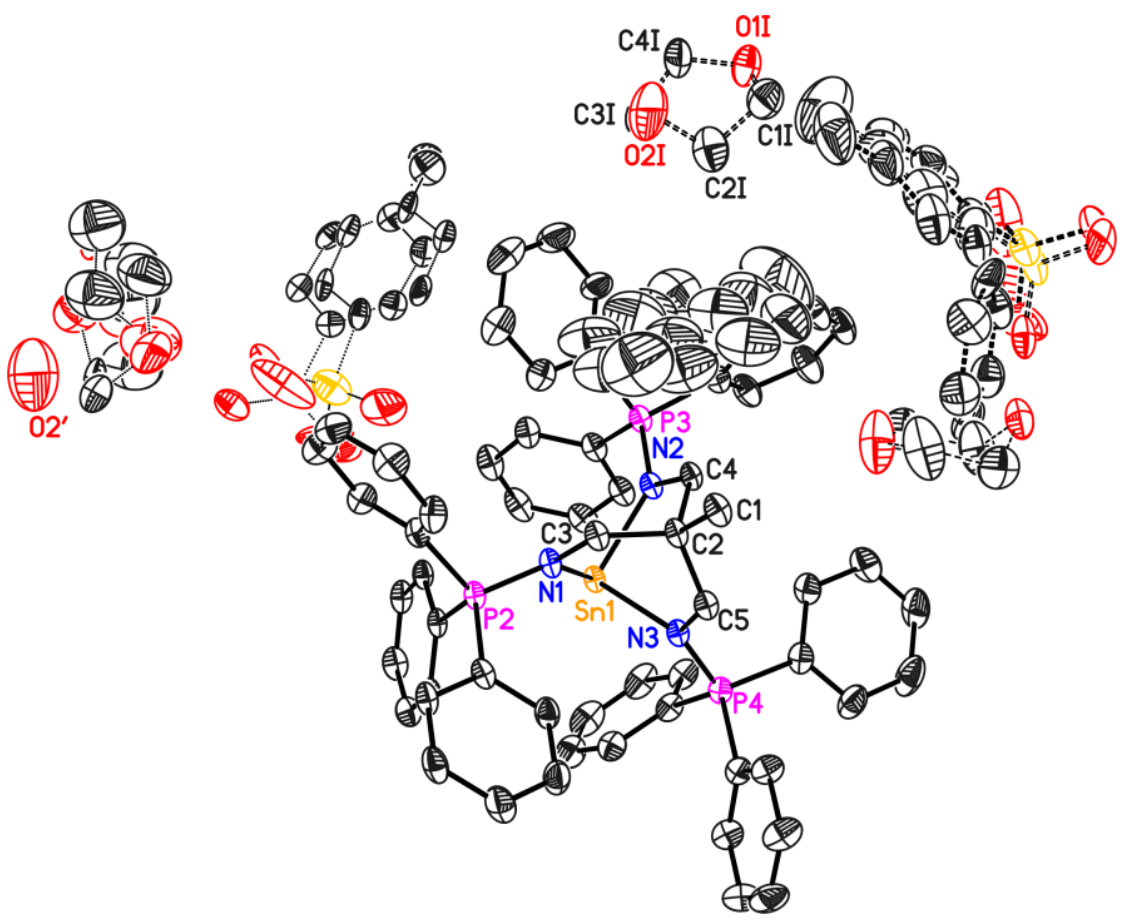

\begin{tabular}{|c|c|c|c|}
\hline Structure code & 21005-snkomplex & Z & 2 \\
\hline Empirical formula & $\mathrm{C}_{87} \mathrm{H}_{90} \mathrm{~N}_{3} \mathrm{O}_{10} \mathrm{P}_{3} \mathrm{~S}_{2} \mathrm{Sn}$ & Crystal size $\left[\mathrm{mm}^{3}\right]$ & $0.3 \times 0.25 \times 0.2$ \\
\hline Formula weight [gmol-1] & 1603.74 & $\rho_{\text {calc }}\left[\mathrm{Mgm}^{-3}\right]$ & 1.393 \\
\hline Temperature $[\mathrm{K}]$ & $100(2)$ & $\mu\left[\mathrm{mm}^{-1}\right]$ & 0.514 \\
\hline Wavelength $[\AA ̊]$ & 0.71073 & $F(000)$ & 1670 \\
\hline Crystal system & Monoclinic & $\Theta$ range $\left[{ }^{\circ}\right]$ & 2.17 to 26.40 \\
\hline Space group & $P \overline{1}$ & Reflections collected & 109559 \\
\hline Unit cell dimensions $[\AA ̊]$ & & Unique reflections & 15644 \\
\hline$a=$ & $10.9564(18)$ & $\mathrm{R}_{\text {int }} / \mathrm{R}_{\sigma}$ & $0.0708 / 0.0377$ \\
\hline$b=$ & $14.820(2)$ & Completeness to $\theta_{\max }$ & $99.8 \%$ \\
\hline$c=$ & $24.610(4)$ & restraints/parameters & 1747 / 1398 \\
\hline$\alpha=$ & $76.204(3)^{\circ}$ & GooF & 1.115 \\
\hline$\beta=$ & $88.086(2)^{\circ}$ & $\mathrm{R} 1[\mathrm{I}>2 \sigma(\mathrm{I})]$ & 0.0495 \\
\hline$\gamma=$ & $80.175(2)^{\circ}$ & wR2 (all data) & 0.1273 \\
\hline Volume $\left[\AA^{3}\right]$ & $3823.6(11)$ & diff. peak / hole $\left[\mathrm{e}^{-3}\right]$ & $1.432 /-1.231$ \\
\hline Resolution [Å] & 0.799 & & \\
\hline
\end{tabular}

The crystal structure of $\mathbf{5}$ consists of the main molecule with Tin(II) coordinated by three nitrogen atoms. The counter ions are two tosylate anions. The starting material was obviously contaminated with tosylate from the azide synthesis. The rest in between turned out to be two dioxane molecules, one toluene molecule and one water molecule. 


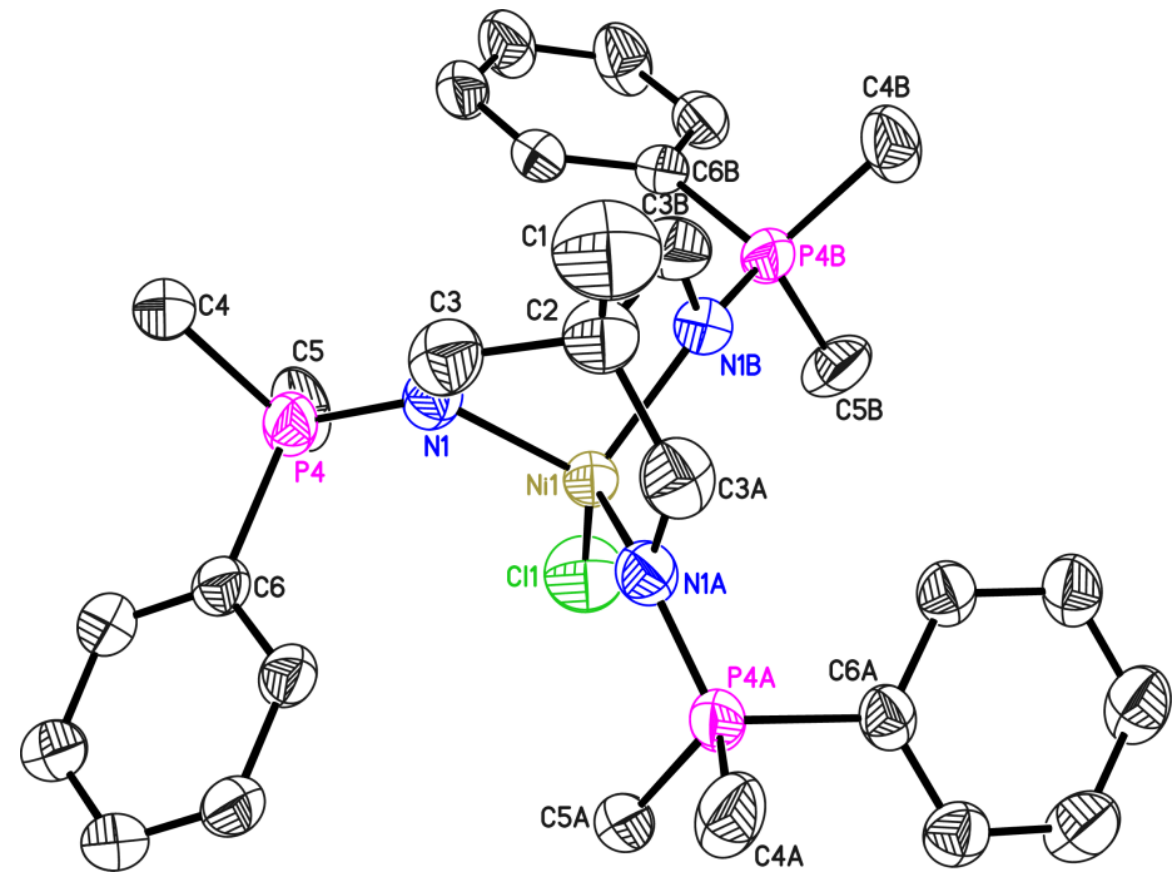

\begin{tabular}{|c|c|c|c|}
\hline Structure code & DK_3004 & Z & 4 \\
\hline Empirical formula & $\mathrm{C}_{161} \mathrm{H}_{219} \mathrm{Cl}_{7} \mathrm{~N}_{15} \mathrm{Ni}_{4} \mathrm{P}_{12}$ & Crystal size $\left[\mathrm{mm}^{3}\right]$ & $0.20 \times 0.20 \times 0.10$ \\
\hline Formula weight [gmol-1] & 3219.14 & $\rho_{\text {calc }}\left[\mathrm{Mgm}^{-3}\right]$ & 1.292 \\
\hline Temperature $[\mathrm{K}]$ & $100(2)$ & $\mu\left[\mathrm{mm}^{-1}\right]$ & 0.731 \\
\hline Wavelength $[\AA ̊]$ & 0.71073 & $F(000)$ & 6804 \\
\hline Crystal system & Cubic & $\Theta$ range $\left[{ }^{\circ}\right]$ & 2.26 to 26.06 \\
\hline Space group & $I \overline{4} 3 d$ & Reflections collected & 110704 \\
\hline Unit cell dimensions $[\AA ̊]$ & & Unique reflections & 2743 \\
\hline$a$ & $25.480(9)$ & $R_{\text {int }} / R_{\sigma}$ & 0.0797 / 0.0182 \\
\hline$b$ & $25.480(9)$ & Completeness to $\theta_{\max }$ & $99.9 \%$ \\
\hline$c$ & $25.480(9)$ & restraints/parameters & $190 / 247$ \\
\hline$\alpha$ & $90^{\circ}$ & GooF & 1.102 \\
\hline$\beta$ & $90^{\circ}$ & $\mathrm{R} 1[\mathrm{I}>2 \sigma(\mathrm{I})]$ & 0.0316 \\
\hline$\gamma$ & $90^{\circ}$ & wR2 (all data) & 0.0841 \\
\hline Volume $\left[\AA^{3}\right]$ & $16543(17)$ & diff. peak / hole $\left[\mathrm{e}^{-3}\right]$ & 0.189 and -0.256 \\
\hline Resolution $[\AA ̊]$ & 0.809 & Flack $\mathrm{x}$ & $0.006(7)$ \\
\hline
\end{tabular}

One disordered Toluene, one acetonitrile molecule and a chlorine counter ion were omitted. 


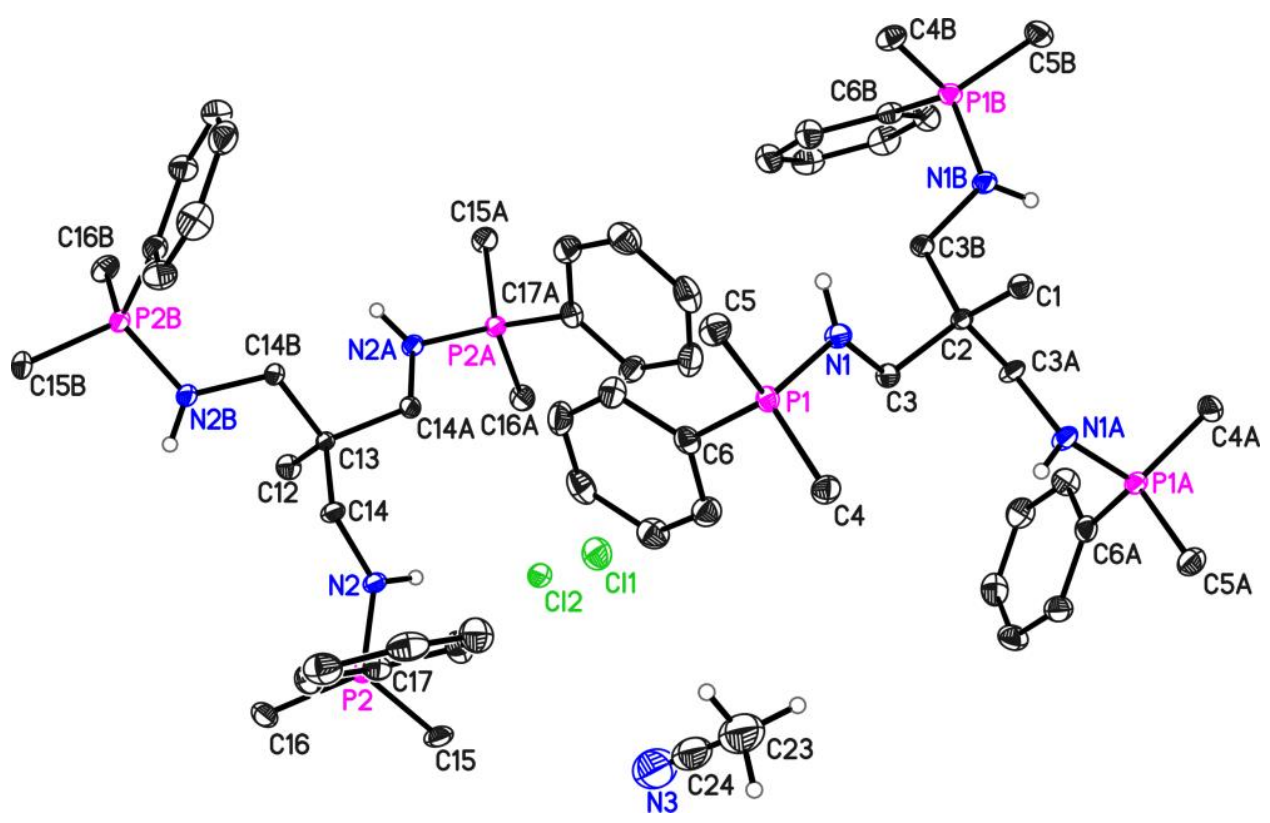

\begin{tabular}{|c|c|c|c|}
\hline Structure code & DK_30011 (5) & $\mathrm{Z}$ & 2 \\
\hline Empirical formula & $\mathrm{C}_{60} \mathrm{H}_{93} \mathrm{Cl}_{6} \mathrm{~N}_{7} \mathrm{P}_{6}$ & Crystal size $\left[\mathrm{mm}^{3}\right]$ & $0.3 \times 0.25 \times 0.2$ \\
\hline Formula weight [gmol-1] & 1310.93 & $\rho_{\text {calc }}\left[\mathrm{Mgm}^{-3}\right]$ & 1.309 \\
\hline Temperature $[\mathrm{K}]$ & $100(2)$ & $\mu\left[\mathrm{mm}^{-1}\right]$ & 0.446 \\
\hline Wavelength $[\AA]$ & 0.71073 & $F(000)$ & 1388 \\
\hline Crystal system & Trigonal & $\Theta$ range $\left[{ }^{\circ}\right]$ & 1.658 to 27.885 \\
\hline Space group & P31c & Reflections collected & 34536 \\
\hline Unit cell dimensions $[\AA ̊]$ & & Unique reflections & 5352 \\
\hline$a=$ & $12.507(4)$ & $\mathrm{R}_{\text {int }} / \mathrm{R}_{\sigma}$ & $0.0592 / 0.0390$ \\
\hline$b=$ & $12.507(4)$ & Completeness to $\theta_{\max }$ & $100.0 \%$ \\
\hline$c=$ & $24.561(9)$ & restraints/parameters & $365 / 287$ \\
\hline$\alpha=$ & $90^{\circ}$ & GooF & 1.044 \\
\hline$\beta=$ & $90^{\circ}$ & $\mathrm{R} 1[\mathrm{I}>2 \sigma(\mathrm{I})]$ & 0.0308 \\
\hline$\gamma=$ & $120^{\circ}$ & wR2 (all data) & 0.0727 \\
\hline Volume $\left[\AA^{3}\right]$ & $3327(3)$ & diff. peak / hole $\left[\mathrm{e} \AA^{-3}\right]$ & 0.224 and -0.252 \\
\hline Resolution $[\AA]$ & 0.760 & Flack $\mathrm{x}$ & $0.01(3)$ \\
\hline
\end{tabular}

The asymmetric unit contains one third of the two main molecules each with one chlorine counter ion each and one acetonitrile molecule. This means each molecule is protonated by one third. Both ligand backbones are disordered over two positions with $15 \%$ occupation (C1, C2, C3, $\mathrm{N} 1)$ and $25 \%$ occupation (C12, C13, C14, N2). 


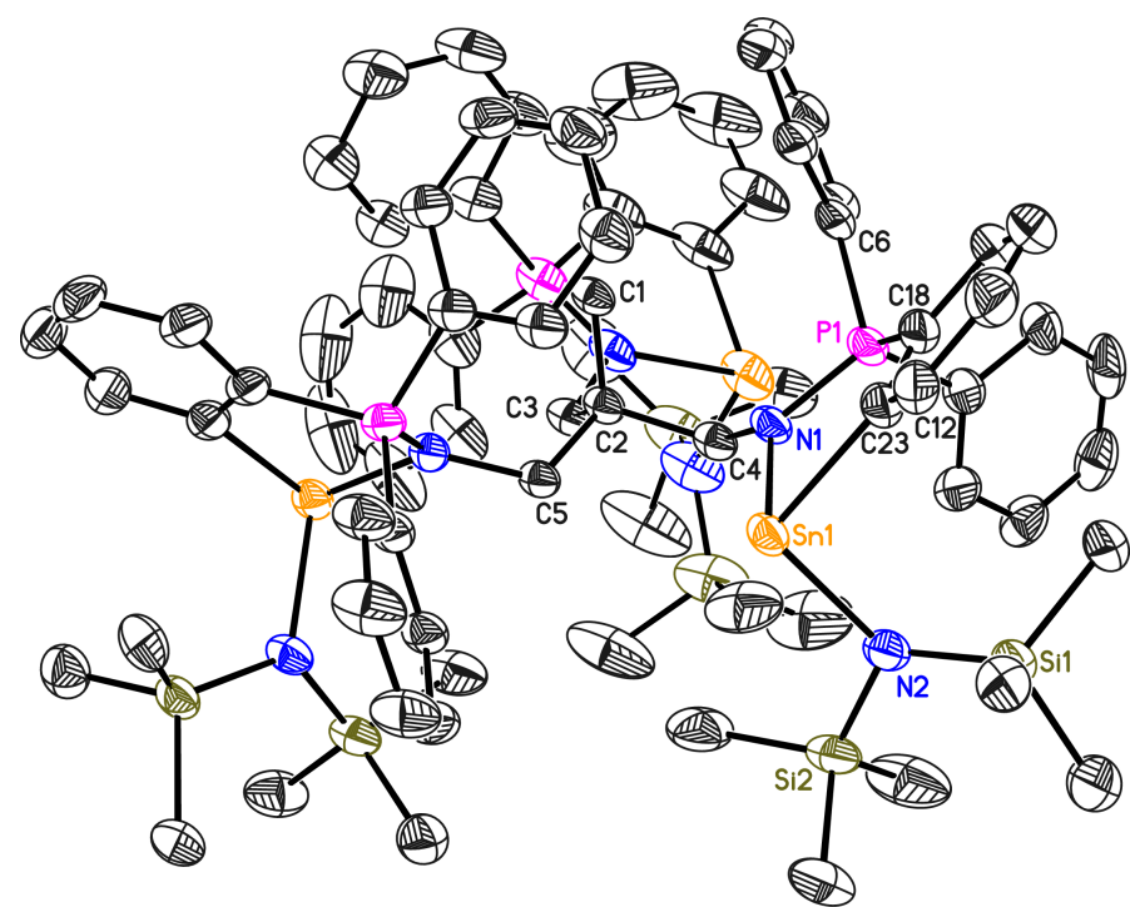

\begin{tabular}{|c|c|c|c|}
\hline Structure code & DK_210014 & z & 4 \\
\hline Empirical formula & $\mathrm{C}_{104} \mathrm{H}_{136.5} \mathrm{~N}_{6} \mathrm{O}_{0.5} \mathrm{P}_{3} \mathrm{Si}_{6} \mathrm{Sn}_{3}$ & Crystal size $\left[\mathrm{mm}^{3}\right]$ & $0.21 \times 0.20 \times 0.20$ \\
\hline Formula weight [gmol-1] & 2096.23 & $\rho_{\text {calc }}\left[\mathrm{Mgm}^{-3}\right]$ & 1.304 \\
\hline Temperature $[\mathrm{K}]$ & $100(2)$ & $\mu\left[\mathrm{mm}^{-1}\right]$ & 0.855 \\
\hline Wavelength $[\AA ̊]$ & 0.71073 & $F(000)$ & 4342 \\
\hline Crystal system & Monoclinic & $\Theta$ range $\left[{ }^{\circ}\right]$ & 1.696 to 25.713 \\
\hline Space group & $P 2_{1} / n$ & Reflections collected & 240267 \\
\hline Unit cell dimensions $[\AA ̊]$ & & Unique reflections & 20300 \\
\hline$a=$ & $14.0624(14)$ & $R_{\text {int }} / R_{\sigma}$ & $0.0476 / 0.0216$ \\
\hline$b=$ & $32.433(3)$ & Completeness to $\theta_{\max }$ & $100.0 \%$ \\
\hline$c=$ & $23.760(2)$ & restraints/parameters & $2673 / 1564$ \\
\hline$\alpha=$ & $90^{\circ}$ & GooF & 1.100 \\
\hline$\beta=$ & $99.811(2)^{\circ}$ & $\mathrm{R} 1[\mathrm{l}>2 \sigma(\mathrm{l})]$ & 0.0380 \\
\hline$\gamma=$ & $90^{\circ}$ & wR2 (all data) & 0.0953 \\
\hline Volume $\left[\AA^{3}\right]$ & 10678.0(18) & diff. peak / hole $\left[\mathrm{e}^{-3}\right]$ & 1.293 and -2.345 \\
\hline Resolution $[\AA ̊]$ & 0.819 & & \\
\hline
\end{tabular}

The asymmetric unit contains the main complex and several highly disordered toluene and dioxane solvent molecules. 


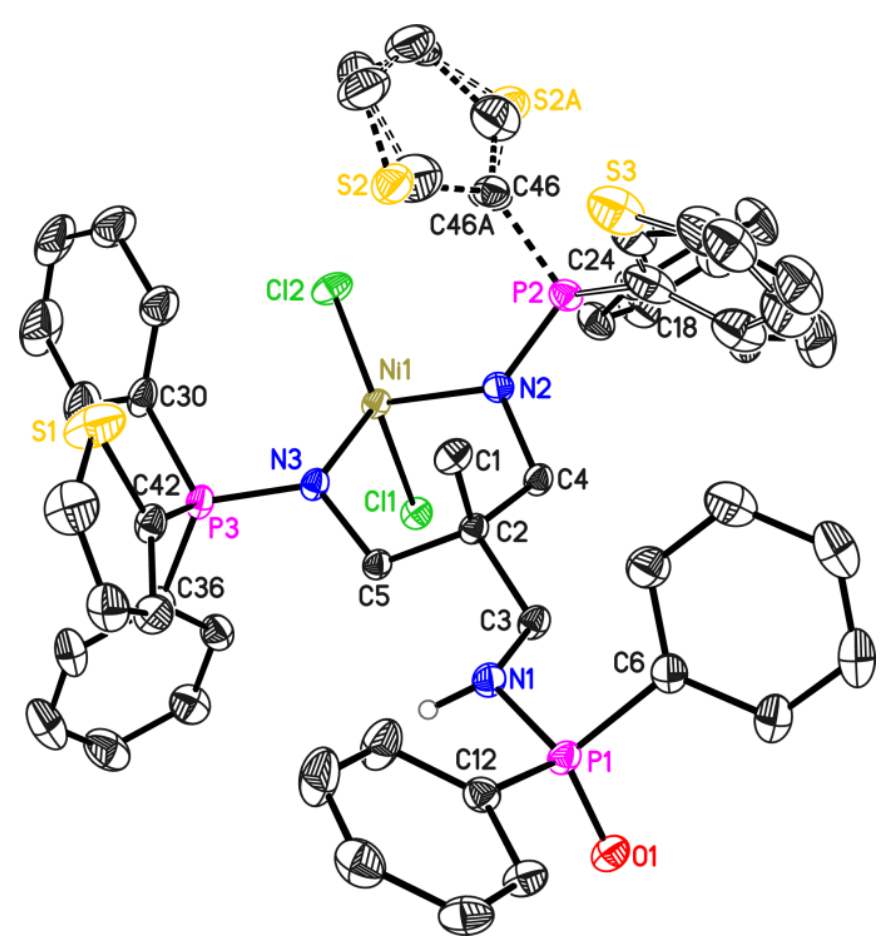

\begin{tabular}{|c|c|c|c|}
\hline Structure code & kristall1 / CV_4025 & Z & 2 \\
\hline Empirical formula & $\mathrm{C}_{61.4} \mathrm{H}_{61.5} \mathrm{Cl}_{2} \mathrm{~N}_{6.1} \mathrm{NiOP}_{3} \mathrm{~S} 2.1$ & Crystal size $\left[\mathrm{mm}^{3}\right]$ & $0.18 \times 0.10 \times 0.10$ \\
\hline Formula weight [gmol-1] & 1189.93 & $\rho_{\text {calc }}\left[\mathrm{Mgm}^{-3}\right]$ & 1.302 \\
\hline Temperature $[\mathrm{K}]$ & $100(2)$ & $\mu\left[\mathrm{mm}^{-1}\right]$ & 0.604 \\
\hline Wavelength $[\AA ̊]$ & 0.71073 & $F(000)$ & 1242 \\
\hline Crystal system & Triclinic & $\Theta$ range $\left[{ }^{\circ}\right]$ & 1.490 to 27.507 \\
\hline Space group & $P \overline{1}$ & Reflections collected & 87297 \\
\hline Unit cell dimensions $[\AA ̊]$ & & Unique reflections & 13901 \\
\hline$a=$ & $11.3750(8)$ & $R_{\text {int }} / R_{\sigma}$ & $0.0367 / 0.0216$ \\
\hline$b=$ & $14.1723(10)$ & Completeness to $\theta_{\max }$ & $99.9 \%$ \\
\hline$c=$ & $19.5507(14)$ & restraints/parameters & 1024 / 889 \\
\hline$\alpha=$ & $102.911(3)$ & GooF & 1.031 \\
\hline$\beta=$ & $92.146(4)$ & $\mathrm{R} 1[\mathrm{I}>2 \sigma(\mathrm{I})]$ & 0.0364 \\
\hline$\gamma=$ & $97.783(4)$ & wR2 (all data) & 0.0989 \\
\hline Volume $\left[\AA^{3}\right]$ & $3036.3(4)$ & diff. peak / hole $\left[\mathrm{e} \AA^{-3}\right]$ & 0.835 and -0.686 \\
\hline Resolution $[\AA ̊]$ & 0.769 & & \\
\hline
\end{tabular}

The asymmetric contains the main complex and disordered Acetonitrile as well as one partly occupied toluene solvent molecule. The thiophene ring with S2 is disordered over two positions. The ring with $\mathrm{S} 3$ is disordered over two positions and partly occupied by a phenyl group. 


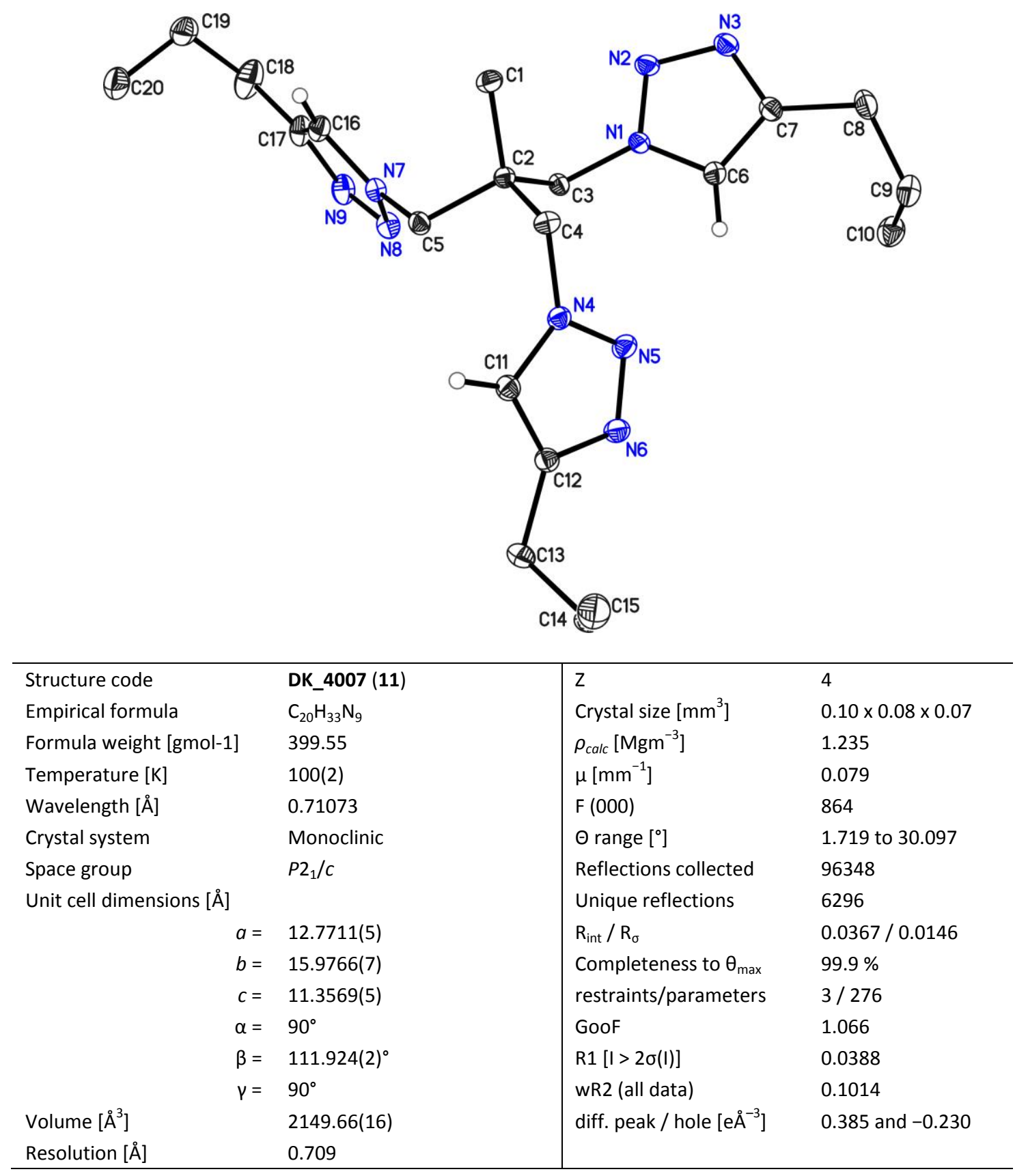




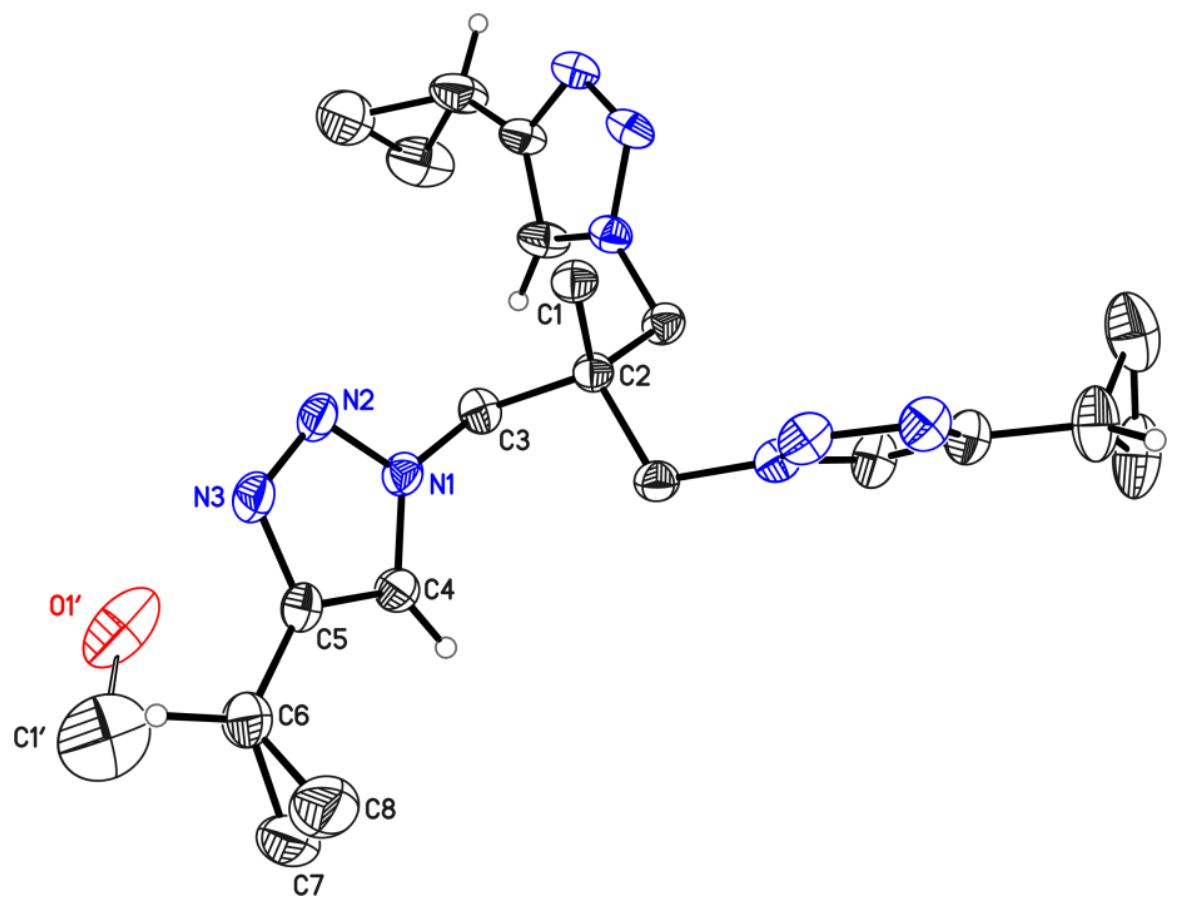

\begin{tabular}{|c|c|c|c|}
\hline Structure code & DK_4008 (12) & Z & 6 \\
\hline Empirical formula & $\mathrm{C}_{20.17} \mathrm{H}_{27.67} \mathrm{~N}_{9} \mathrm{O}_{0.17}$ & Crystal size $\left[\mathrm{mm}^{3}\right]$ & $0.32 \times 0.28 \times 0.22$ \\
\hline Formula weight [gmol-1] & 398.84 & $\rho_{\text {calc }}\left[\mathrm{Mgm}^{-3}\right]$ & 1.201 \\
\hline Temperature $[\mathrm{K}]$ & $100(2)$ & $\mu\left[\mathrm{mm}^{-1}\right]$ & 0.078 \\
\hline Wavelength $[\AA ̊]$ & 0.71073 & $F(000)$ & 1278 \\
\hline Crystal system & Trigonal & $\Theta$ range $\left[{ }^{\circ}\right]$ & 2.490 to 29.101 \\
\hline Space group & $R 3 c$ & Reflections collected & 70287 \\
\hline Unit cell dimensions $[\AA ̊]$ & & Unique reflections & 1992 \\
\hline$a=$ & $12.021(6)$ & $R_{\text {int }} / R_{\sigma}$ & 0.0390 / 0.0117 \\
\hline$b=$ & $12.021(6)$ & Completeness to $\theta_{\max }$ & $99.9 \%$ \\
\hline$c=$ & $26.434(8)$ & restraints/parameters & $21 / 108$ \\
\hline$\alpha=$ & $90^{\circ}$ & GooF & 1.121 \\
\hline$\beta=$ & $90^{\circ}$ & $\mathrm{R} 1[\mathrm{I}>2 \sigma(\mathrm{I})]$ & 0.0391 \\
\hline$\gamma=$ & $120^{\circ}$ & wR2 (all data) & 0.1173 \\
\hline Volume $\left[\AA^{3}\right]$ & $3308(3)$ & diff. peak / hole $\left[\mathrm{e}^{-3}\right]$ & 0.225 and -0.170 \\
\hline Resolution $[\AA ̊]$ & 0.731 & Flack $x$ & $-0.2(3)$ \\
\hline
\end{tabular}

The absolute structure could not be determined reliably because of the low anomalous signal. The methanol molecule is located on a threefold axis with $6 \%$ occupation. 


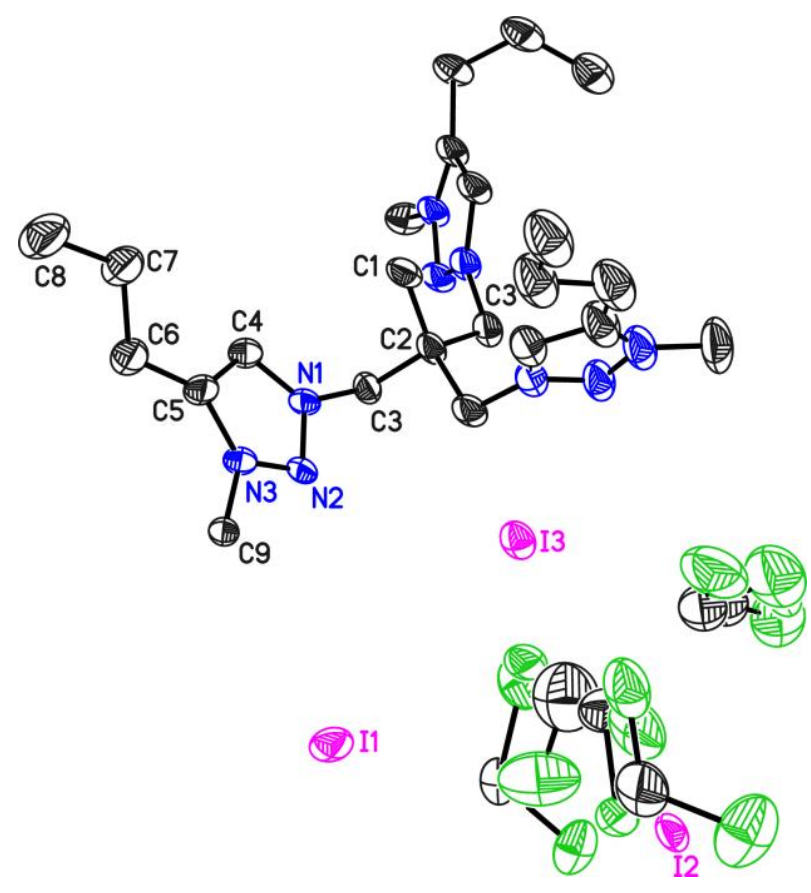

\begin{tabular}{|c|c|c|c|}
\hline Structure code & DK_6005 (15) & Z & 4 \\
\hline Empirical formula & $\mathrm{C}_{25.10} \mathrm{H}_{46.20} \mathrm{Cl}_{4.21} \mathrm{I}_{3} \mathrm{~N}_{9}$ & Crystal size $\left[\mathrm{mm}^{3}\right]$ & $0.34 \times 0.15 \times 0.14$ \\
\hline Formula weight [gmol-1] & 1004.05 & $\rho_{\text {calc }}\left[\mathrm{Mgm}^{-3}\right]$ & 1.594 \\
\hline Temperature $[\mathrm{K}]$ & $100(2)$ & $\mu\left[\mathrm{mm}^{-1}\right]$ & 2.537 \\
\hline Wavelength $[\AA ̊]$ & 0.71073 & $F(000)$ & 1961 \\
\hline Crystal system & Monoclinic & $\Theta$ range $\left[{ }^{\circ}\right]$ & 1.433 to 29.689 \\
\hline Space group & $P 2_{1} / c$ & Reflections collected & 208035 \\
\hline Unit cell dimensions $[\AA ̊]$ & & Unique reflections & 8919 \\
\hline$a=$ & $12.0251(11)$ & $R_{\text {int }} / R_{\sigma}$ & $0.0570 / 0.0180$ \\
\hline$b=$ & $28.413(2)$ & Completeness to $\theta_{\max }$ & $98.7 \%$ \\
\hline$c=$ & $13.2136(11)$ & restraints/parameters & $1659 / 628$ \\
\hline$\alpha=$ & $90^{\circ}$ & GooF & 1.174 \\
\hline$\beta=$ & $112.054(3)^{\circ}$ & $\mathrm{R} 1[\mathrm{I}>2 \sigma(\mathrm{I})]$ & 0.0744 \\
\hline$\gamma=$ & $90^{\circ}$ & wR2 (all data) & 0.1697 \\
\hline Volume $\left[\AA^{3}\right]$ & $4184.4(6)$ & diff. peak / hole $\left[\mathrm{e}^{-3}\right]$ & 1.514 and -1.693 \\
\hline Resolution $[\AA ̊]$ & 0.717 & & \\
\hline
\end{tabular}

Each branch of the three-membered ligand was found to be disordered over two positions. Two dichloromethane molecules were found to be disordered over six positions. 


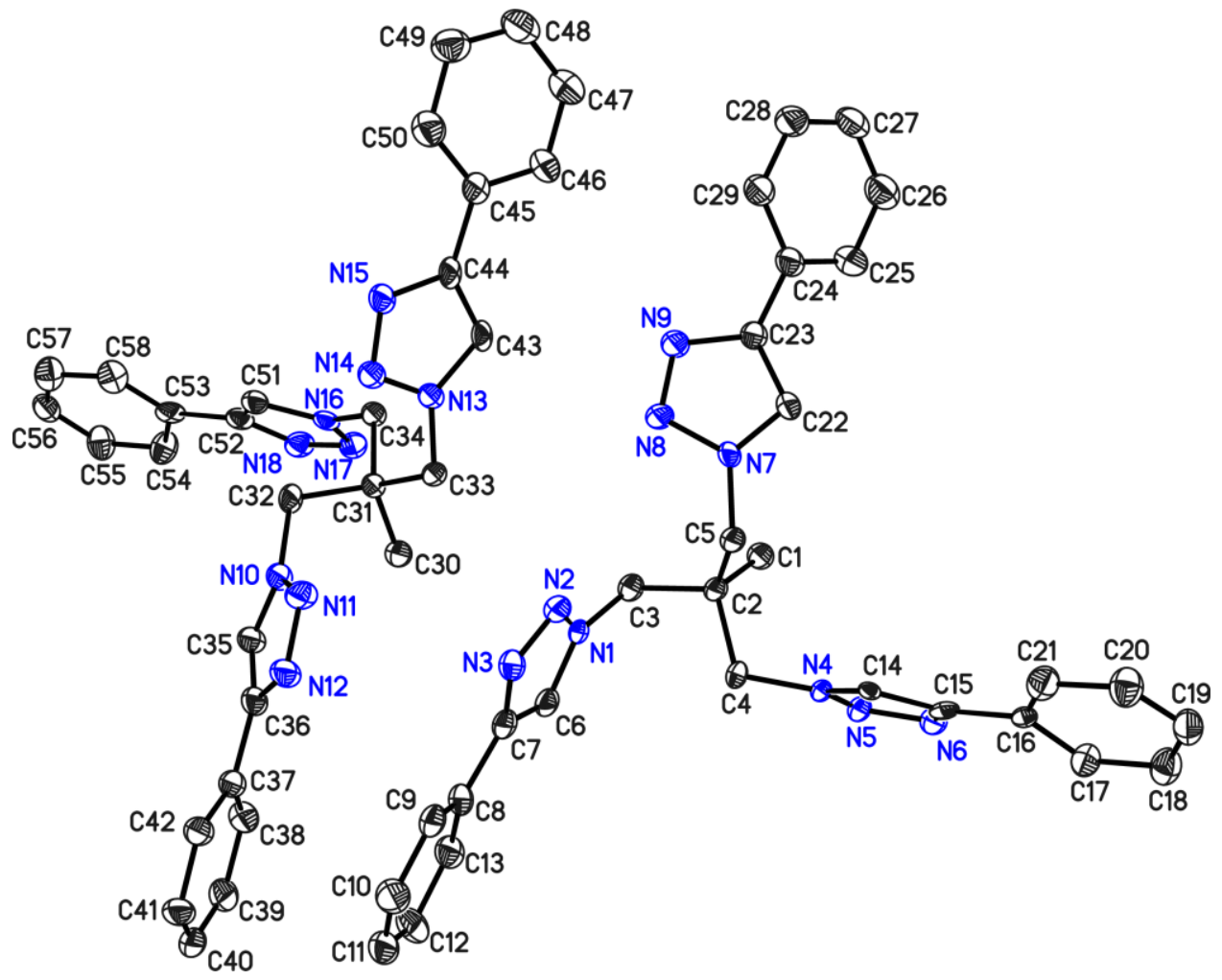

\begin{tabular}{|c|c|c|c|}
\hline Structure code & DK_4006 (12) & Z & 4 \\
\hline Empirical formula & $\mathrm{C}_{29} \mathrm{H}_{27} \mathrm{~N}_{9}$ & Crystal size $\left[\mathrm{mm}^{3}\right]$ & $0.1 \times 0.1 \times 0.05$ \\
\hline Formula weight [gmol-1] & 501.59 & $\rho_{\text {calc }}\left[\mathrm{Mgm}^{-3}\right]$ & 1.343 \\
\hline Temperature $[\mathrm{K}]$ & $100(2)$ & $\mu\left[\mathrm{mm}^{-1}\right]$ & 0.085 \\
\hline Wavelength $[\AA ̊]$ & 0.71073 & $F(000)$ & 1056 \\
\hline Crystal system & Triclinic & $\Theta$ range $\left[{ }^{\circ}\right]$ & 1.236 to 23.255 \\
\hline Space group & $P \overline{1}$ & Reflections collected & 54589 \\
\hline Unit cell dimensions $[\AA ̊]$ & & Unique reflections & 7147 \\
\hline$a=$ & $5.9616(5)$ & $\mathrm{R}_{\text {int }} / \mathrm{R}_{\sigma}$ & $0.0633 / 0.0383$ \\
\hline$b=$ & $16.7221(14)$ & Completeness to $\theta_{\max }$ & $99.8 \%$ \\
\hline$c=$ & $25.4001(19)$ & restraints/parameters & 1134 / 687 \\
\hline$\alpha=$ & $83.565(6)^{\circ}$ & GooF & 1.131 \\
\hline$\beta=$ & $83.915(4)^{\circ}$ & $\mathrm{R} 1[\mathrm{I}>2 \sigma(\mathrm{I})]$ & 0.1332 \\
\hline$\gamma=$ & $81.791(4)^{\circ}$ & wR2 (all data) & 0.3615 \\
\hline Volume $\left[\AA^{3}\right]$ & $2480.0(4)$ & diff. peak / hole $\left[\mathrm{e} \AA^{-3}\right]$ & 0.998 and -0.474 \\
\hline Resolution $[\AA ̊]$ & 0.900 & & \\
\hline
\end{tabular}

The asymmetric contains two molecules. Due to not optimal crystal quality the residual density was higher than normal but the desired features of the structure could be identified unambiguously. 


\section{Crystallographic Cooperation}

This chapter deals with the crystallographic measurements which were performed in cooperation with other research groups.

\subsection{Structures Measured for Prof. Swadhin K. Mandal}

\subsubsection{Published Structures}

\begin{tabular}{ll}
$\begin{array}{l}\text { CCDC number / } \\
\text { structure code }\end{array}$ & Reference \\
\hline $\begin{array}{l}\text { not in database } \\
\text { DK_SKMMeNNMeCu }\end{array}$ & A. Mukherjee, T. K. Sen, S. Mandal, D. Kratzert, A. Döring, C. Schulzke, J. Chem. Sci. 2011, 123, \\
\hline $\mathbf{8 0 5 5 7 9}$ & T. K. Sen, A. Mukherjee, A. Modak, P. K. Ghorai, D. Kratzert, M. Granitzka, D. Stalke, S. K. \\
DK_SMAIMe2NN & Mandal, Chem. Eur. J. 2012, 18, 54-58. \\
$\mathbf{8 0 5 5 7 7}$ & \\
DK_SMOOAIMe2 & \\
$\mathbf{8 0 5 5 7 8}$ & \\
DK_SMiPrNNAIMe2 & \\
\hline
\end{tabular}

\subsubsection{Unpublished Structures}

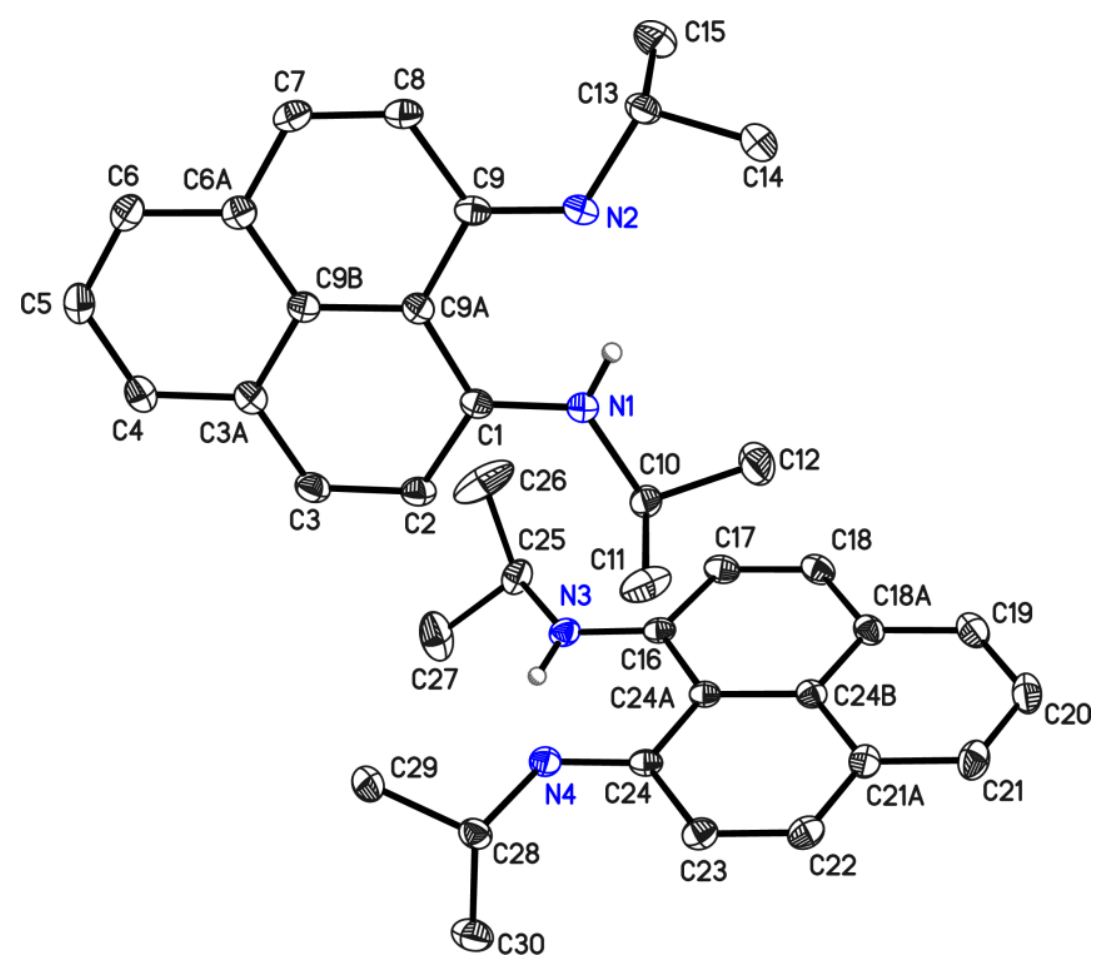

\begin{tabular}{ll|ll}
\hline Structure code & DK_SKMiprnniprCu_ligand & Z & 8 \\
Empirical formula & $\mathrm{C}_{19} \mathrm{H}_{22} \mathrm{~N}_{2}$ & Crystal size $\left[\mathrm{mm}^{3}\right]$ & $0.18 \times 0.18 \times 0.07$ \\
Formula weight [gmol-1] & 278.39 & $\rho_{\text {calc }}\left[\mathrm{Mgm}^{-3}\right]$ & 1.192 \\
Temperature $[\mathrm{K}]$ & $100(2)$ & $\mu\left[\mathrm{mm}^{-1}\right]$ & 0.070 \\
Wavelength $[\AA]$ & $\mathrm{F}(000)$ & 1200 \\
Crystal system & 0.71073 & $\Theta$ range $\left[{ }^{\circ}\right]$ & 2.07 to 27.88 \\
Space group & Monoclinic & Reflections collected & 37848
\end{tabular}




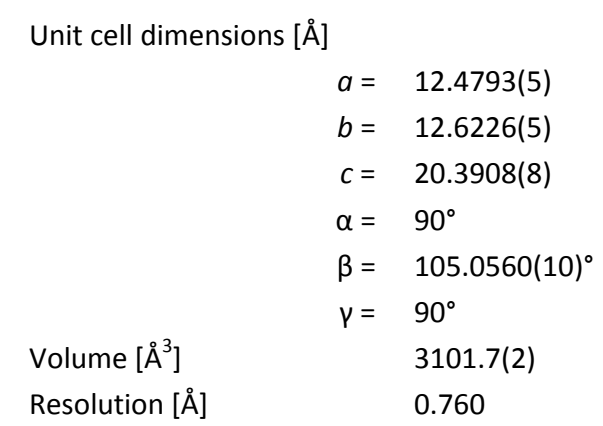

\begin{tabular}{|ll} 
Unique reflections & 3708 \\
$\mathrm{R}_{\text {int }} / \mathrm{R}_{\sigma}$ & $0.0338 / 0.0156$ \\
Completeness to $\theta_{\max }$ & $100.0 \%$ \\
restraints/parameters & $6 / 401$ \\
GooF & 1.042 \\
$\mathrm{R} 1[\mathrm{I}>2 \sigma(\mathrm{I})]$ & 0.0330 \\
WR2 (all data) & 0.0897 \\
diff. peak / hole $\left[\mathrm{e} \AA^{-3}\right]$ & $0.260 /-0.183$ \\
Flack $x$ & - \\
\hline
\end{tabular}

The absolute structure could not be determined because of the low anomalous signal of the included atoms with Mo-Radiation. The hydrogen atoms $\mathrm{H} 1$ and $\mathrm{H} 2$ were each found on both nitrogen atoms of each molecule. The occupancy of $\mathrm{H} 1$ vs. $\mathrm{H} 1$ ' and $\mathrm{H} 2 \mathrm{vs}$. $\mathrm{H}_{2}$ ' were refined with a free variable. The positions of these hydrogen atoms were found by difference Fourier synthesis and the positions were freely refined with a "DFIX 0.010 .91 " restraint.

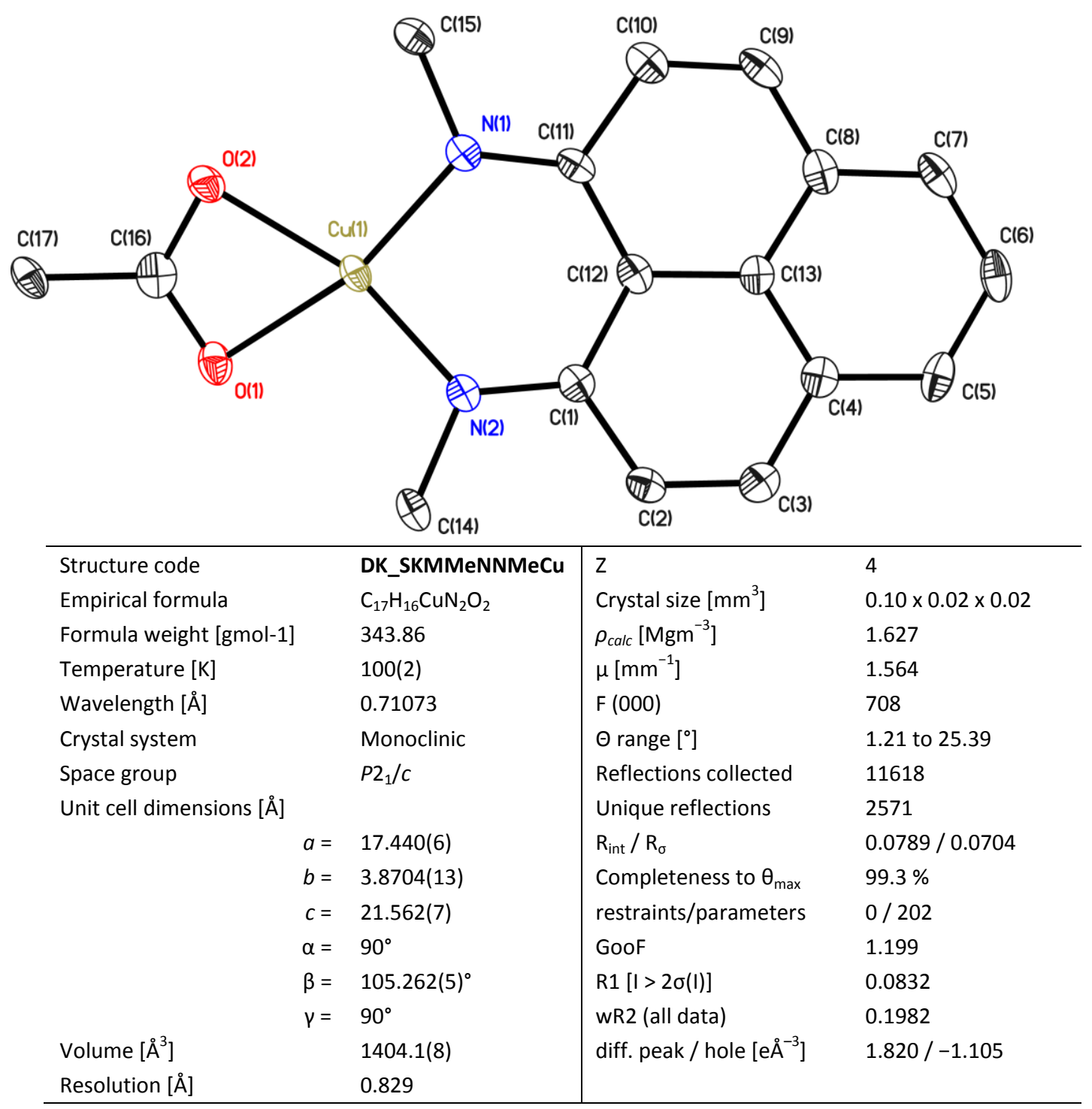

This structure was published in the Journal of Chemical Science, but without knowledge of the coauthors and without supplementing of the cif file to the CSD and very few crystallographic information in the paper. 


\subsection{Structures Measured for Prof. Peter Jones}

\subsubsection{Published structures}

\begin{tabular}{ll} 
CCDC number & Reference \\
\hline $\mathbf{7 6 9 8 4 2}$ & C. Woelper, M. D. P. Bastardes, I. Dix, D. Kratzert, P. G. Jones, Z. Naturforsch., B: Chem. Sci. 2010, 65, \\
Morse & 647-673.
\end{tabular}

\subsection{Structures Measured for Sakya S. Sen (Prof. H. W. Roesky)}

\subsubsection{Published Structures}

\begin{tabular}{|c|c|}
\hline $\begin{array}{l}\text { CCDC number / } \\
\text { structure code }\end{array}$ & Reference \\
\hline 762176 & R. S. Ghadwal, S. S. Sen, H. W. Roesky, M. Granitzka, D. Kratzert, S. Merkel, D. Stalke, Angew. \\
\hline DK_SA07 & Chem., Int. Ed. 2010, 49, 3952-3955. \\
\hline 762177 & S. S. Sen, G. Tavcar, H. W. Roesky, D. Kratzert, J. Hey, D. Stalke, Organometallics 2010, 29, 2343- \\
\hline DK_SA08 & 2347. \\
\hline 778534 & S. S. Sen, R. S. Ghadwal, D. Kratzert, D. Stern, H. W. Roesky, D. Stalke, Organometallics 2011, 30, \\
\hline DK_SA09 & 1030-1033. \\
\hline 767704 & S. S. Sen, D. Kratzert, D. Stern, H. W. Roesky, D. Stalke, Inorg. Chem. 2010, 49, 5786-5788. \\
\hline \multicolumn{2}{|l|}{ DK_SA12_new } \\
\hline 781628 & S. Khan, S. S. Sen, D. Kratzert, G. Tavcar, H. W. Roesky, D. Stalke, Chem. Eur. J. 2011, 17, 4283-4290. \\
\hline \multicolumn{2}{|l|}{ DK_SA13 } \\
\hline $7 \overline{78537}$ & G. Tavcar, S. S. Sen, H. W. Roesky, J. Hey, D. Kratzert, D. Stalke, Organometallics 2010, 29, 3930- \\
\hline DK_SA14 & 3935. \\
\hline 843608 & S. S. Sen, J. Hey, D. Kratzert, H. W. Roesky, D. Stalke, Organometallics 2012, 31, 435-439. \\
\hline \multicolumn{2}{|l|}{ DK_SA16 } \\
\hline 782243 & G. Tavcar, S. S. Sen, H. W. Roesky, J. Hey, D. Kratzert, D. Stalke, Organometallics 2010, 29, 3930- \\
\hline DK_SA21 & 3935. \\
\hline 793275 & S. S. Sen, S. Khan, H. W. Roesky, D. Kratzert, K. Meindl, J. Henn, D. Stalke, J.-P. Demers, A. Lange, \\
\hline DK_SA25A & Angew. Chem., Int. Ed. 2011, 50, 2322-2325. \\
\hline 807092 & S. S. Sen, S. Khan, D. Kratzert, H. W. Roesky, D. Stalke, Eur. J. Inorg. Chem. 2011, 1370-1373. \\
\hline \multicolumn{2}{|r|}{ 要, } \\
\hline 819818 & S. Khan, S. S. Sen, R. Michel, D. Kratzert, H. W. Roesky, D. Stalke, Organometallics 2011, 30, 2643- \\
\hline $\begin{array}{c}\text { See work of Reent } \\
\text { Michel }\end{array}$ & 2645. \\
\hline
\end{tabular}




\subsubsection{Unpublished Structures}

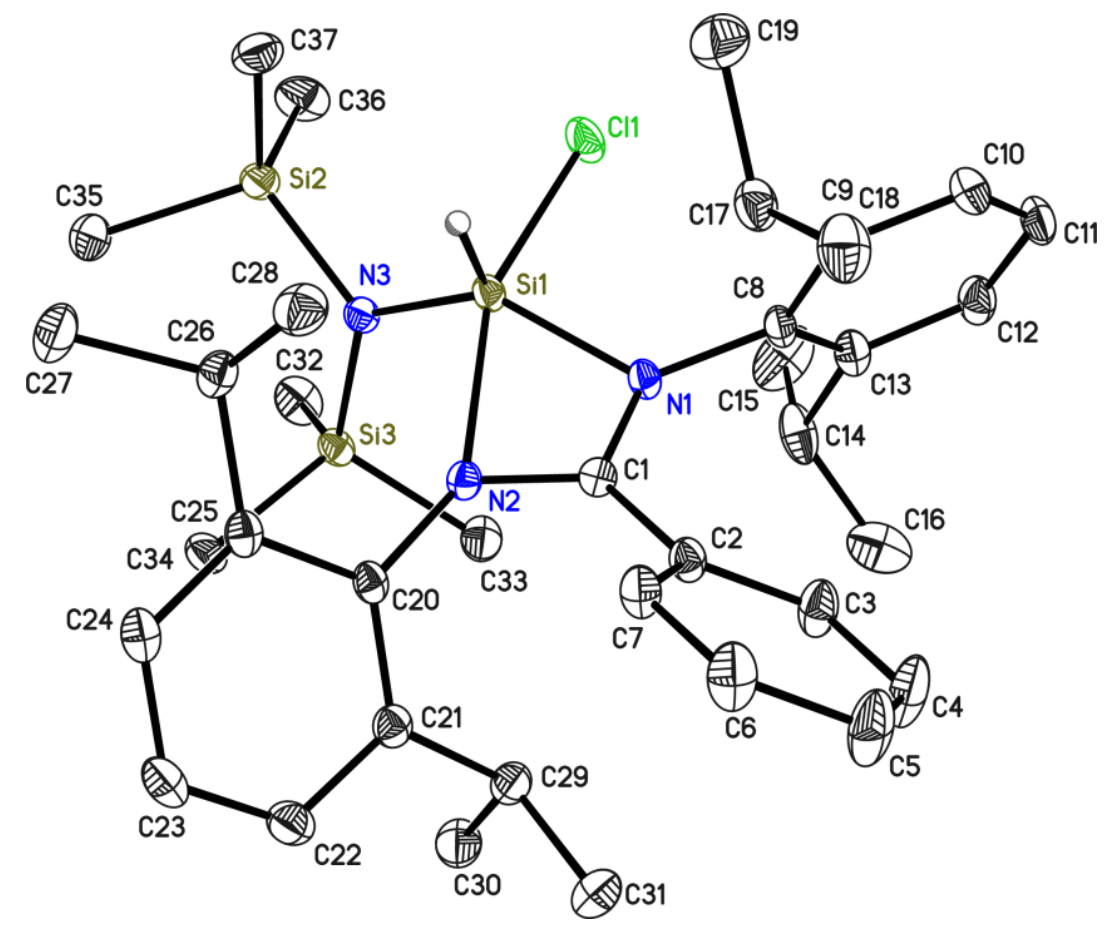

\begin{tabular}{|c|c|c|c|}
\hline Structure code & DK_SA03 & Z & 4 \\
\hline Empirical formula & $\mathrm{C}_{37} \mathrm{H}_{58} \mathrm{CIN}_{3} \mathrm{Si}_{3}$ & Crystal size $\left[\mathrm{mm}^{3}\right]$ & $0.25 \times 0.20 \times 0.10$ \\
\hline Formula weight [gmol-1] & 664.58 & $\rho_{\text {calc }}\left[\mathrm{Mgm}^{-3}\right]$ & 1.129 \\
\hline Temperature $[\mathrm{K}]$ & $100(2)$ & $\mu\left[\mathrm{mm}^{-1}\right]$ & 0.218 \\
\hline Wavelength $[\AA ̊]$ & 0.71073 & $F(000)$ & 1440 \\
\hline Crystal system & Monoclinic & $\Theta$ range $\left[{ }^{\circ}\right]$ & 1.596 to 27.892 \\
\hline Space group & $P 2_{1} / n$ & Reflections collected & 32212 \\
\hline Unit cell dimensions $[\AA ̊]$ & & Unique reflections & 9331 \\
\hline$a=$ & $12.0054(7)$ & $\mathrm{R}_{\text {int }} / \mathrm{R}_{\sigma}$ & $0.0226 / 0.0218$ \\
\hline$b=$ & $18.2581(10)$ & Completeness to $\theta_{\max }$ & $99.9 \%$ \\
\hline$c=$ & $18.6808(11)$ & restraints/parameters & 82 / 414 \\
\hline$\alpha=$ & $90^{\circ}$ & GooF & 1.033 \\
\hline$\beta=$ & $107.3090(10)^{\circ}$ & $\mathrm{R} 1[\mathrm{I}>2 \sigma(\mathrm{I})]$ & 0.0331 \\
\hline$\gamma=$ & $90^{\circ}$ & wR2 (all data) & 0.0909 \\
\hline Volume $\left[\AA^{3}\right]$ & 3909.3(4) & diff. peak / hole $\left[\mathrm{e}^{-3}\right]$ & $0.407 /-0.315$ \\
\hline Resolution $[\AA ̊]$ & 0.760 & & \\
\hline
\end{tabular}

The hydrogen atom $\mathrm{H} 1$ was found by difference Fourier analysis and its position was refined freely with a distance restraint. The isotropic motion of $\mathrm{H} 1$ was constrained 1.6 times to the $\mathrm{U}_{\text {eq }}{ }^{-}$ value of Si1. 


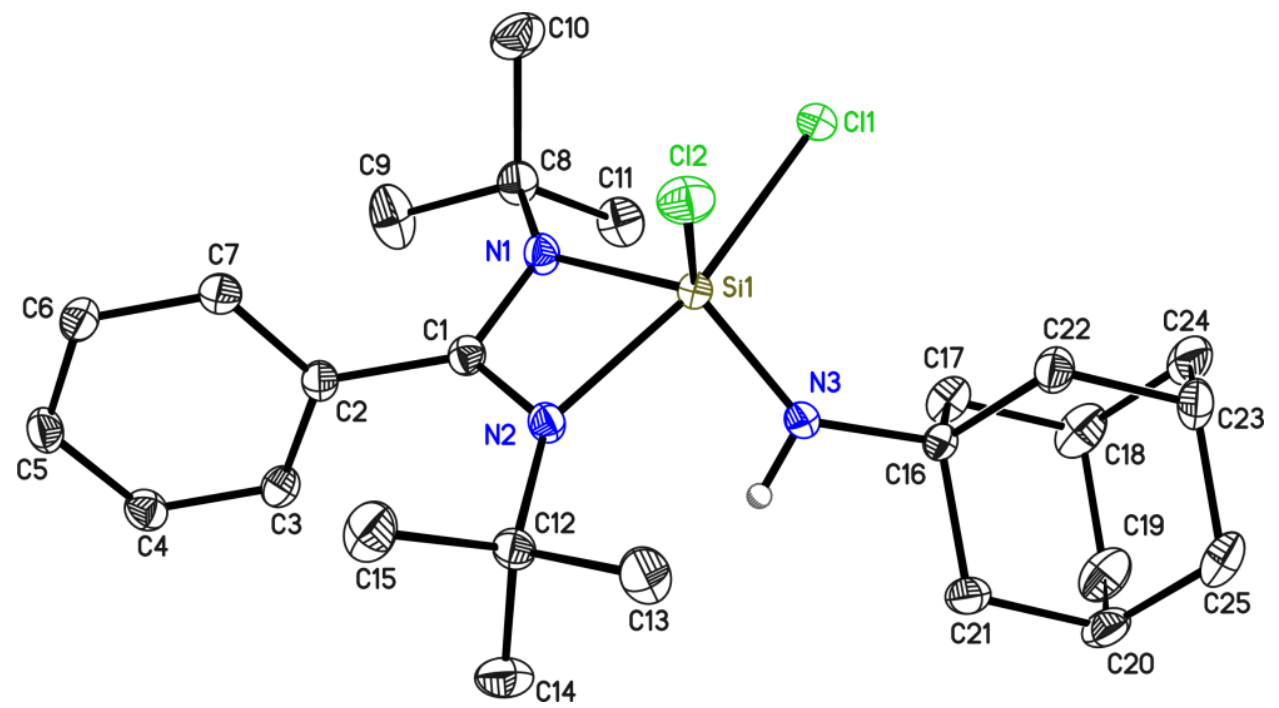

\begin{tabular}{|c|c|c|c|}
\hline Structure code & DK_SA15 & $z$ & 4 \\
\hline Empirical formula & $\mathrm{C}_{25} \mathrm{H}_{39} \mathrm{Cl}_{2} \mathrm{~N}_{3} \mathrm{Si}$ & Crystal size $\left[\mathrm{mm}^{3}\right]$ & $0.18 \times 0.15 \times 0.14$ \\
\hline Formula weight [gmol-1] & 480.58 & $\rho_{\text {calc }}\left[\mathrm{Mgm}^{-3}\right]$ & 1.251 \\
\hline Temperature $[\mathrm{K}]$ & $100(2)$ & $\mu\left[\mathrm{mm}^{-1}\right]$ & 0.320 \\
\hline Wavelength $[\AA ̊]$ & 0.71073 & $F(000)$ & 1032 \\
\hline Crystal system & Monoclinic & $\Theta$ range $\left[{ }^{\circ}\right]$ & 1.72 to 27.55 \\
\hline Space group & $P 2_{1} / c$ & Reflections collected & 57975 \\
\hline Unit cell dimensions $[\AA ̊]$ & & Unique reflections & 5856 \\
\hline$a$ & $12.3433(5)$ & $\mathrm{R}_{\text {int }} / \mathrm{R}_{\sigma}$ & $0.0375 / 0.0188$ \\
\hline$b=$ & $16.8493(6)$ & Completeness to $\theta_{\max }$ & $99.8 \%$ \\
\hline$c=$ & $12.7271(5)$ & restraints/parameters & $1 / 289$ \\
\hline$\alpha=$ & $90^{\circ}$ & GooF & 1.032 \\
\hline$\beta=$ & $105.861(2)$ & $\mathrm{R} 1[\mathrm{I}>2 \sigma(\mathrm{I})]$ & 0.0302 \\
\hline$\gamma=$ & $90^{\circ}$ & wR2 (all data) & 0.0780 \\
\hline Volume $\left[\AA^{3}\right]$ & $2546.16(17)$ & diff. peak / hole $\left[\mathrm{e}^{-3}\right]$ & $0.392 /-0.211$ \\
\hline Resolution $[\AA]$ & 0.768 & & \\
\hline
\end{tabular}

The hydrogen atom H3' was found by difference Fourier analysis and its position was refined freely with a distance restraint. The isotropic motion of $\mathrm{H}^{\prime}$ ' was constrained 1.2 times to the $\mathrm{U}_{\mathrm{eq}^{-}}$ value of $\mathrm{N3}$.

\subsection{Structures Measured for Shabana Khan (Prof. H. W. Roesky)}

\subsubsection{Published Structures}

\begin{tabular}{cl}
$\begin{array}{c}\text { CCDC number / } \\
\text { Structure code }\end{array}$ & Reference \\
\hline $\mathbf{7 7 9 0 9 6}$ & S. Khan, S. S. Sen, H. W. Roesky, D. Kratzert, R. Michel, D. Stalke, Inorg. Chem. 2010, 49, 9689-9693. \\
SK_01 & \\
\hline $\mathbf{7 8 1 6 2 9}$ & S. Khan, S. S. Sen, D. Kratzert, G. Tavcar, H. W. Roesky, D. Stalke, Chem. Eur. J. 2011, 17, 4283-4290. \\
DK_SK08 & \\
$\mathbf{7 8 1 6 3 1}$ & \\
DK_SK24 & \\
\hline $\mathbf{7 9 3 2 7 6}$ & S. S. Sen, S. Khan, H. W. Roesky, D. Kratzert, K. Meindl, J. Henn, D. Stalke, J.-P. Demers, A. Lange, \\
DK_SK13 & Angew. Chem., Int. Ed. 2011, 50, 2322-2325. \\
\hline
\end{tabular}




\subsubsection{Unpublished Structures}

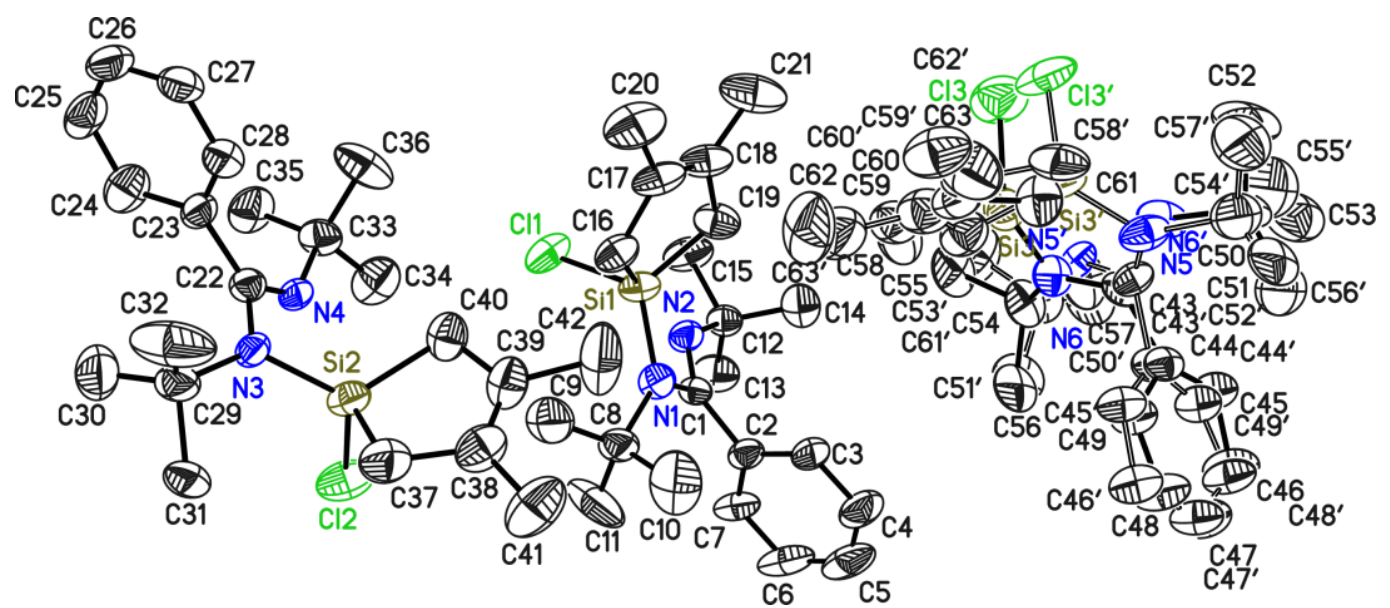

\begin{tabular}{|c|c|c|c|}
\hline Structure code & DK_SK11 & Z & 12 \\
\hline Empirical formula & $\mathrm{C}_{21} \mathrm{H}_{33} \mathrm{ClN}_{2} \mathrm{Si}$ & Crystal size $\left[\mathrm{mm}^{3}\right]$ & $0.2 \times 0.18 \times 0.05$ \\
\hline Formula weight $\left[\mathrm{gmol}^{-1}\right]$ & 377.03 & $\rho_{\text {calc }}\left[\mathrm{Mgm}^{-3}\right]$ & 1.184 \\
\hline Temperature $[\mathrm{K}]$ & $100(2)$ & $\mu\left[\mathrm{mm}^{-1}\right]$ & 0.244 \\
\hline Wavelength $[\AA ̊]$ & 0.71073 & $F(000)$ & 2448 \\
\hline Crystal system & Monoclinic & $\Theta$ range $\left[{ }^{\circ}\right]$ & 1.482 to 25.437 \\
\hline Space group & $P 2_{1} / n$ & Reflections collected & 98262 \\
\hline Unit cell dimensions $[\AA ̊]$ & & Unique reflections & 11712 \\
\hline$a=$ & $11.243(4)$ & $\mathrm{R}_{\mathrm{int}} / \mathrm{R}_{\sigma}$ & $0.0800 / 0.0429$ \\
\hline$b=$ & $13.738(4)$ & Completeness to $\theta_{\max }$ & $99.7 \%$ \\
\hline$c=$ & $41.082(13)$ & restraints/parameters & $1365 / 914$ \\
\hline$\alpha=$ & $90^{\circ}$ & GooF & 1.047 \\
\hline$\beta=$ & $90^{\circ}$ & $\mathrm{R} 1[\mathrm{I}>2 \sigma(\mathrm{I})]$ & 0.0521 \\
\hline$\gamma=$ & $90^{\circ}$ & wR2 (all data) & 0.1368 \\
\hline Volume $\left[\AA^{3}\right]$ & $6345(3)$ & diff. peak / hole $\left[\mathrm{e}^{-3}\right]$ & $0.261 /-0.282$ \\
\hline Resolution $[\AA ̊]$ & 0.827 & & \\
\hline
\end{tabular}

The structure turned out to refine better in $P 2_{1} / n$ with the twin law $-1000-10001$ instead of $P n a 2_{1}$. One of the three molecules is disordered over two positions with $50 \%$ occupation each. The bond lengths of the disordered molecule were made similar to the ordered molecules.

\subsection{Structures Measured for Nina Tölle (Prof. L. F. Tietze)}

\subsubsection{Published Structures}

\begin{tabular}{cl}
$\begin{array}{l}\text { CCDC number / } \\
\text { structure code }\end{array}$ & Reference \\
\hline $\mathbf{7 4 5 3 3 0}$ & L. F. Tietze, N. Tolle, D. Kratzert, D. Stalke, Org. Lett. 2009, 11, 5230-5233. \\
DK_NTD103a & \\
$\mathbf{7 4 5 3 2 9}$ & \\
DK_NTE03A & \\
\hline
\end{tabular}




\subsubsection{Unpublished Structures}

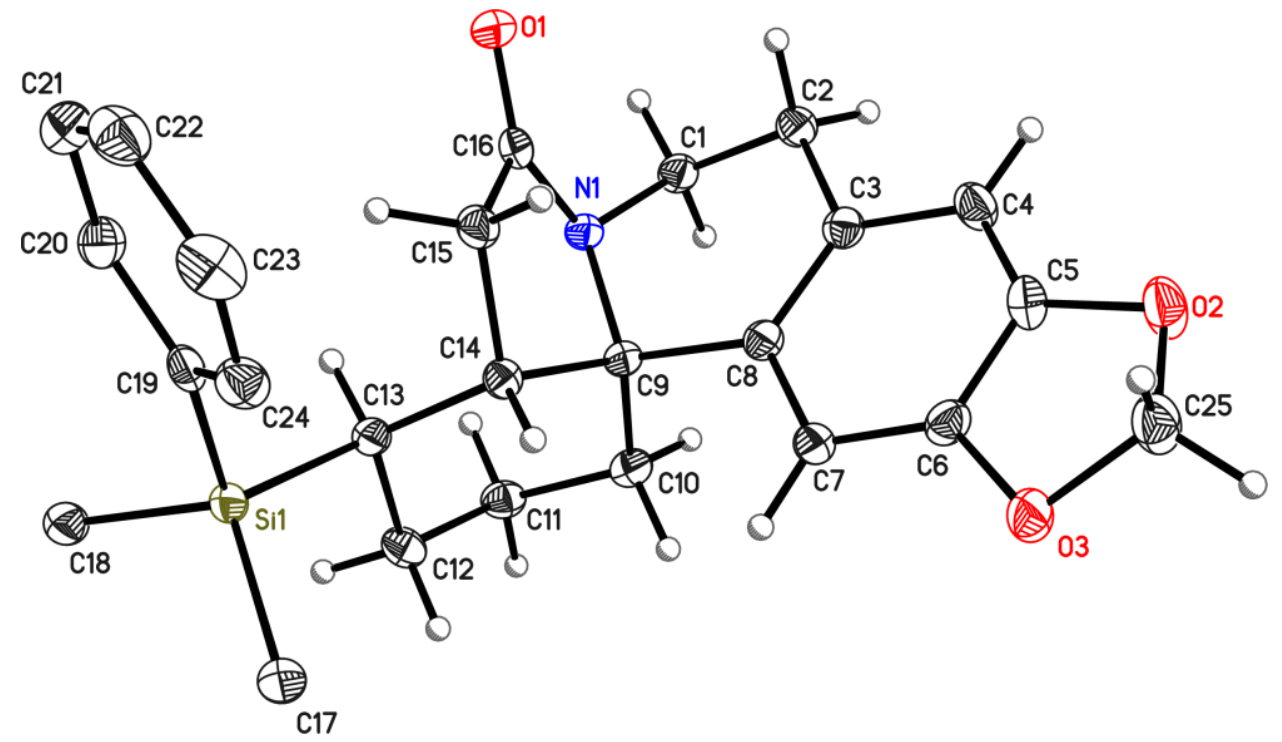

\begin{tabular}{|c|c|c|c|}
\hline Structure code & DK_NTD45a & Z & 4 \\
\hline Empirical formula & $\mathrm{C}_{25} \mathrm{H}_{29} \mathrm{NO}_{3} \mathrm{Si}$ & Crystal size $\left[\mathrm{mm}^{3}\right]$ & $0.18 \times 0.15 \times 0.10$ \\
\hline Formula weight [gmol-1] & 419.58 & $\rho_{\text {calc }}\left[\mathrm{Mgm}^{-3}\right]$ & 1.297 \\
\hline Temperature $[\mathrm{K}]$ & $100(2)$ & $\mu\left[\mathrm{mm}^{-1}\right]$ & 0.136 \\
\hline Wavelength $[\AA ̊]$ & 0.71073 & $F(000)$ & 896 \\
\hline Crystal system & Monoclinic & $\Theta$ range $\left[{ }^{\circ}\right]$ & 1.59 to 24.71 \\
\hline Space group & $P 2_{1} / c$ & Reflections collected & 30274 \\
\hline Unit cell dimensions $[\AA ̊]$ & & Unique reflections & 3672 \\
\hline$a=$ & $12.9682(15)$ & $R_{\text {int }} / R_{\sigma}$ & $0.0533 / 0.0274$ \\
\hline$b=$ & $7.2856(8)$ & Completeness to $\theta_{\max }$ & $100.0 \%$ \\
\hline$c=$ & $22.984(3)$ & restraints/parameters & $0 / 273$ \\
\hline$\alpha=$ & $90^{\circ}$ & GooF & 1.031 \\
\hline$\beta=$ & $98.181(2)^{\circ}$ & $\mathrm{R} 1[\mathrm{I}>2 \sigma(\mathrm{I})]$ & 0.0336 \\
\hline$\gamma=$ & $90^{\circ}$ & wR2 (all data) & 0.0812 \\
\hline Volume $\left[\AA^{3}\right]$ & 2149.4(4) & diff. peak / hole $\left[\mathrm{e} \AA^{-3}\right]$ & $0.284 /-0.249$ \\
\hline Resolution $[\AA]$ & 0.850 & & \\
\hline
\end{tabular}

Hydrogen atoms from methyl- and Phenyl groups has been omitted. 


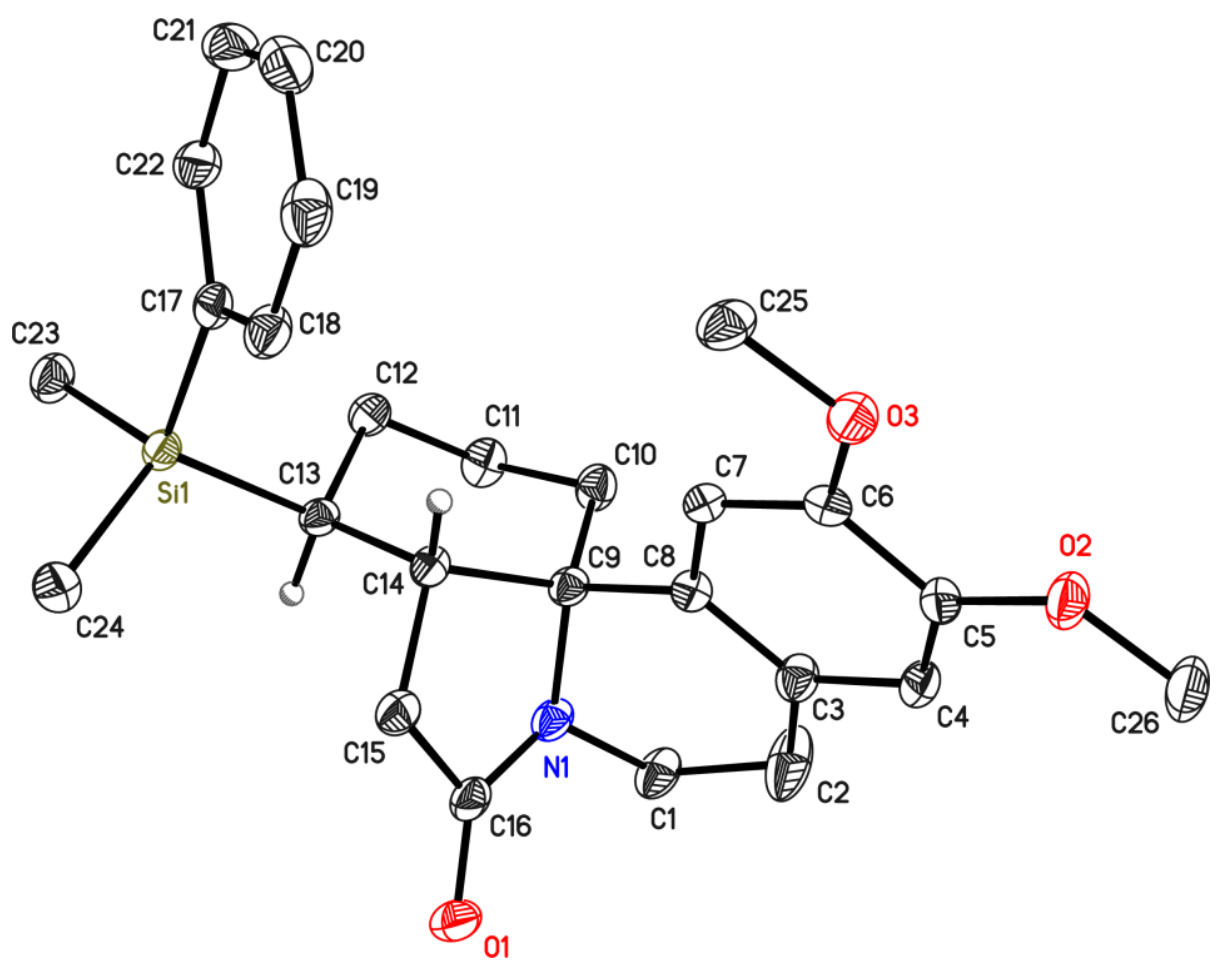

\begin{tabular}{|c|c|c|c|}
\hline Structure code & DK_NTD51a & $\mathrm{Z}$ & 4 \\
\hline Empirical formula & $\mathrm{C}_{26} \mathrm{H}_{33} \mathrm{NO}_{3} \mathrm{Si}$ & Crystal size $\left[\mathrm{mm}^{3}\right]$ & $0.25 \times 0.2 \times 0.1$ \\
\hline Formula weight [gmol-1] & 435.62 & $\rho_{\text {calc }}\left[\mathrm{Mgm}^{-3}\right]$ & 1.252 \\
\hline Temperature $[\mathrm{K}]$ & $100(2)$ & $\mu\left[\mathrm{mm}^{-1}\right]$ & 0.129 \\
\hline Wavelength $[\AA ̊]$ & 0.71073 & $F(000)$ & 936 \\
\hline Crystal system & Monoclinic & $\Theta$ range $\left[{ }^{\circ}\right]$ & 1.94 to 25.35 \\
\hline Space group & $P 2_{1} / c$ & Reflections collected & 30274 \\
\hline Unit cell dimensions $[\AA ̊]$ & & Unique reflections & 76055 \\
\hline$a$ & $10.6456(9)$ & $\mathrm{R}_{\text {int }} / \mathrm{R}_{\sigma}$ & $0.0384 / 0.0138$ \\
\hline$b$ & $11.0330(9)$ & Completeness to $\theta_{\max }$ & $100.0 \%$ \\
\hline$c=$ & $19.9214(16)$ & restraints/parameters & $0 / 284$ \\
\hline$\alpha=$ & $90^{\circ}$ & GooF & 1.055 \\
\hline$\beta=$ & $98.9380(10)^{\circ}$ & $\mathrm{R} 1[\mathrm{I}>2 \sigma(\mathrm{I})]$ & 0.0326 \\
\hline$\gamma=$ & $90^{\circ}$ & wR2 (all data) & 0.0876 \\
\hline Volume $\left[\AA^{3}\right]$ & $2311.4(3)$ & diff. peak / hole $\left[\mathrm{e} \AA^{-3}\right]$ & $0.360 /-0.270$ \\
\hline Resolution $[\AA]$ & 0.830 & & \\
\hline
\end{tabular}

Hydrogen atoms except $\mathrm{H} 13$ and $\mathrm{H} 14$ has been omitted. 


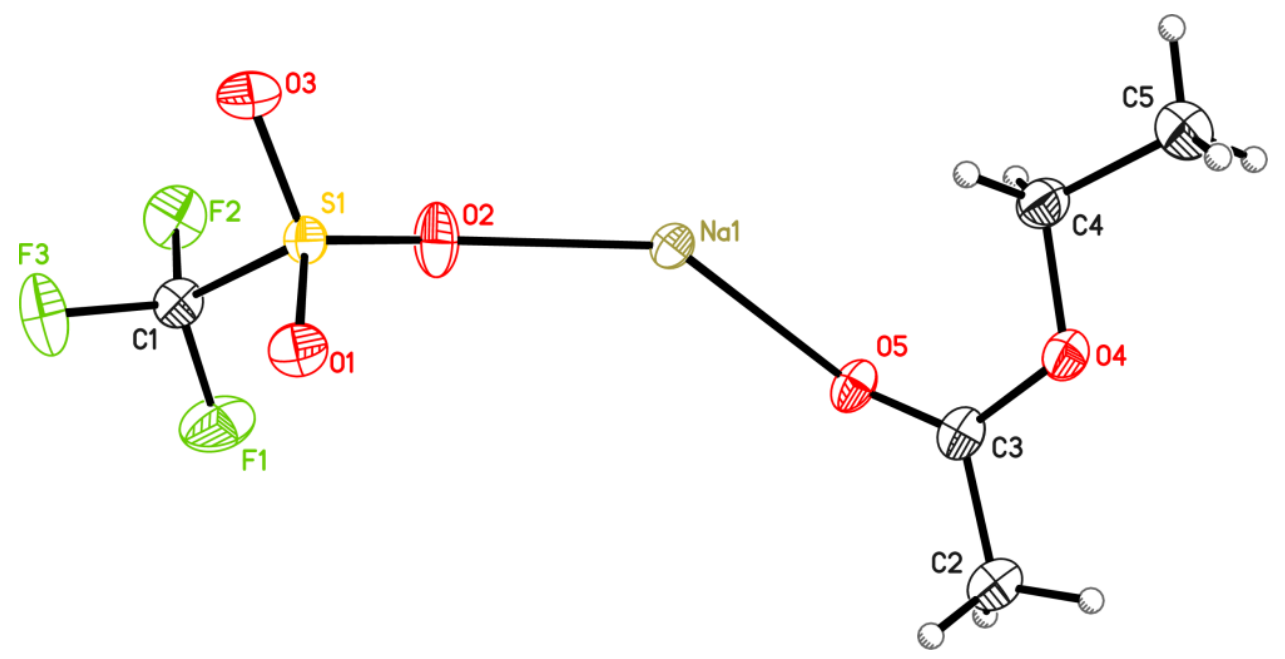

\begin{tabular}{|c|c|c|c|}
\hline Structure code & DK_NTD50b & Z & 2 \\
\hline Empirical formula & $\mathrm{C}_{5} \mathrm{H}_{8} \mathrm{~F}_{3} \mathrm{NaO}_{5} \mathrm{~S}$ & Crystal size $\left[\mathrm{mm}^{3}\right]$ & $0.15 \times 0.10 \times 0.10$ \\
\hline Formula weight [gmol-1] & 260.16 & $\rho_{\text {calc }}\left[\mathrm{Mgm}^{-3}\right]$ & 1.694 \\
\hline Temperature $[\mathrm{K}]$ & $100(2)$ & $\mu\left[\mathrm{mm}^{-1}\right]$ & 0.403 \\
\hline Wavelength $[\AA ̊]$ & 0.71073 & $F(000)$ & 264 \\
\hline Crystal system & Triclinic & $\Theta$ range $\left[{ }^{\circ}\right]$ & 3.411 to 24.751 \\
\hline Space group & $P \overline{1}$ & Reflections collected & 6373 \\
\hline Unit cell dimensions $[\AA ̊]$ & & Unique reflections & 1738 \\
\hline$a$ & $5.8537(13)$ & $\mathrm{R}_{\text {int }} / \mathrm{R}_{\sigma}$ & $0.0162 / 0.0123$ \\
\hline$b$ & $7.9695(17)$ & Completeness to $\theta_{\max }$ & $94.0 \%$ \\
\hline$c$ & $12.001(3)$ & restraints/parameters & $102 / 138$ \\
\hline$\alpha$ & $79.267(3)^{\circ}$ & GooF & 1.078 \\
\hline$\beta$ & $75.914(2)^{\circ}$ & $\mathrm{R} 1[\mathrm{I}>2 \sigma(\mathrm{I})]$ & 0.0260 \\
\hline$\gamma$ & $71.054(2)^{\circ}$ & wR2 (all data) & 0.0684 \\
\hline Volume $\left[\AA^{3}\right]$ & $510.1(2)$ & diff. peak / hole $\left[\mathrm{e} \AA^{-3}\right]$ & $0.539 /-0.398$ \\
\hline Resolution $[\AA ̊]$ & 0.849 & & \\
\hline
\end{tabular}

The structure forms a two dimensional network. 

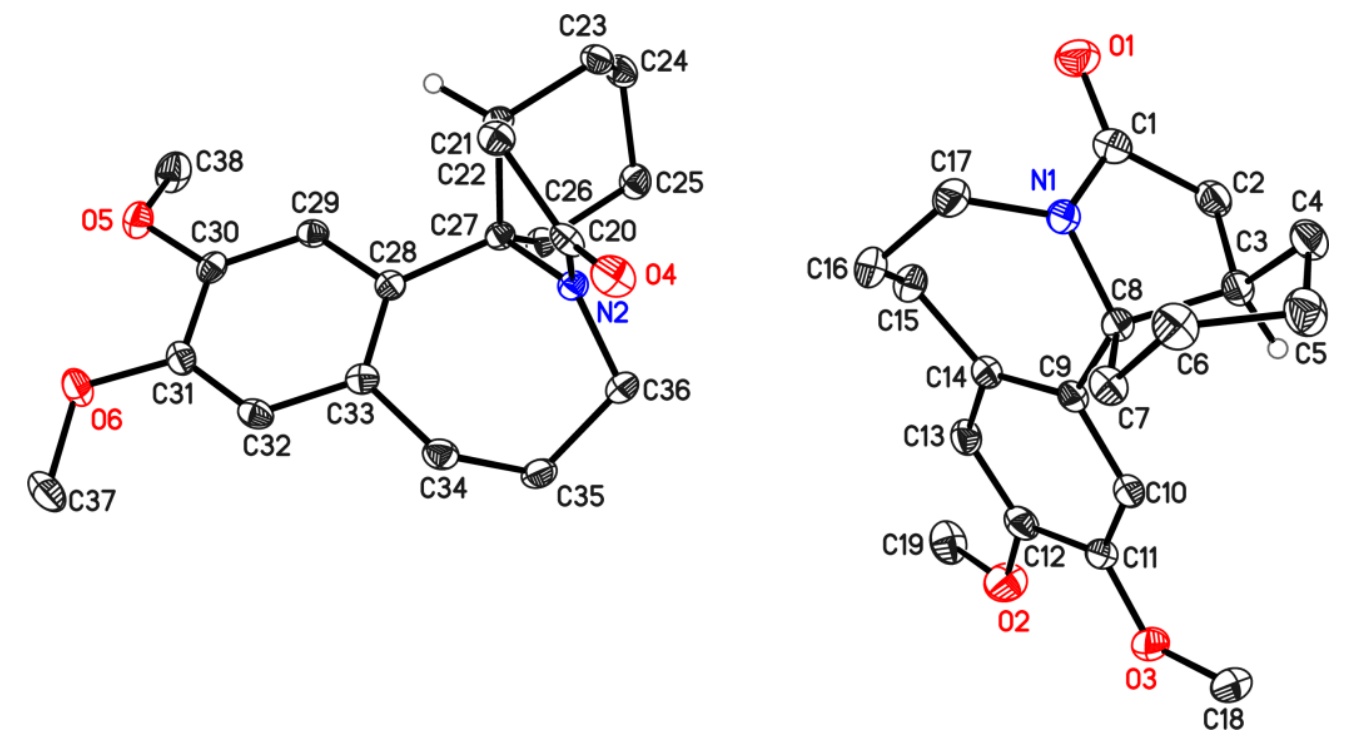

\begin{tabular}{|c|c|c|c|}
\hline Structure code & DK_NTC74a & $Z$ & 8 \\
\hline Empirical formula & $\mathrm{C}_{19} \mathrm{H}_{25} \mathrm{NO}_{3}$ & Crystal size $\left[\mathrm{mm}^{3}\right]$ & $0.15 \times 0.14 \times 0.13$ \\
\hline Formula weight [gmol-1] & 315.40 & $\rho_{\text {calc }}\left[\mathrm{Mgm}^{-3}\right]$ & 1.295 \\
\hline Temperature $[\mathrm{K}]$ & $100(2)$ & $\mu\left[\mathrm{mm}^{-1}\right]$ & 0.087 \\
\hline Wavelength $[\AA ̊]$ & 0.71073 & $F(000)$ & 1360 \\
\hline Crystal system & Monoclinic & $\Theta$ range $\left[{ }^{\circ}\right]$ & 1.00 to 26.78 \\
\hline Space group & $P 2_{1} / c$ & Reflections collected & 69234 \\
\hline Unit cell dimensions $[\AA ̊]$ & & Unique reflections & 6910 \\
\hline$a=$ & $21.698(23)$ & $\mathrm{R}_{\text {int }} / \mathrm{R}_{\sigma}$ & $0.0709 / 0.0310$ \\
\hline$b=$ & $10.5879(11)$ & Completeness to $\theta_{\max }$ & $99.9 \%$ \\
\hline$c=$ & $14.9963(16)$ & restraints/parameters & $0 / 419$ \\
\hline$\alpha=$ & $90^{\circ}$ & GooF & 1.034 \\
\hline$\beta=$ & $110.0840(10)^{\circ}$ & $\mathrm{R} 1[\mathrm{I}>2 \sigma(\mathrm{I})]$ & 0.0407 \\
\hline$\gamma=$ & $90^{\circ}$ & wR2 (all data) & 0.1011 \\
\hline Volume $\left[\AA^{3}\right]$ & $3235.6(10)$ & diff. peak / hole $\left[\mathrm{e} \AA^{-3}\right]$ & $0.311 /-0.252$ \\
\hline Resolution [Å] & 0.789 & & \\
\hline
\end{tabular}

All hydrogen atoms except $\mathrm{H} 5$ and $\mathrm{H} 21$ have been omitted. 


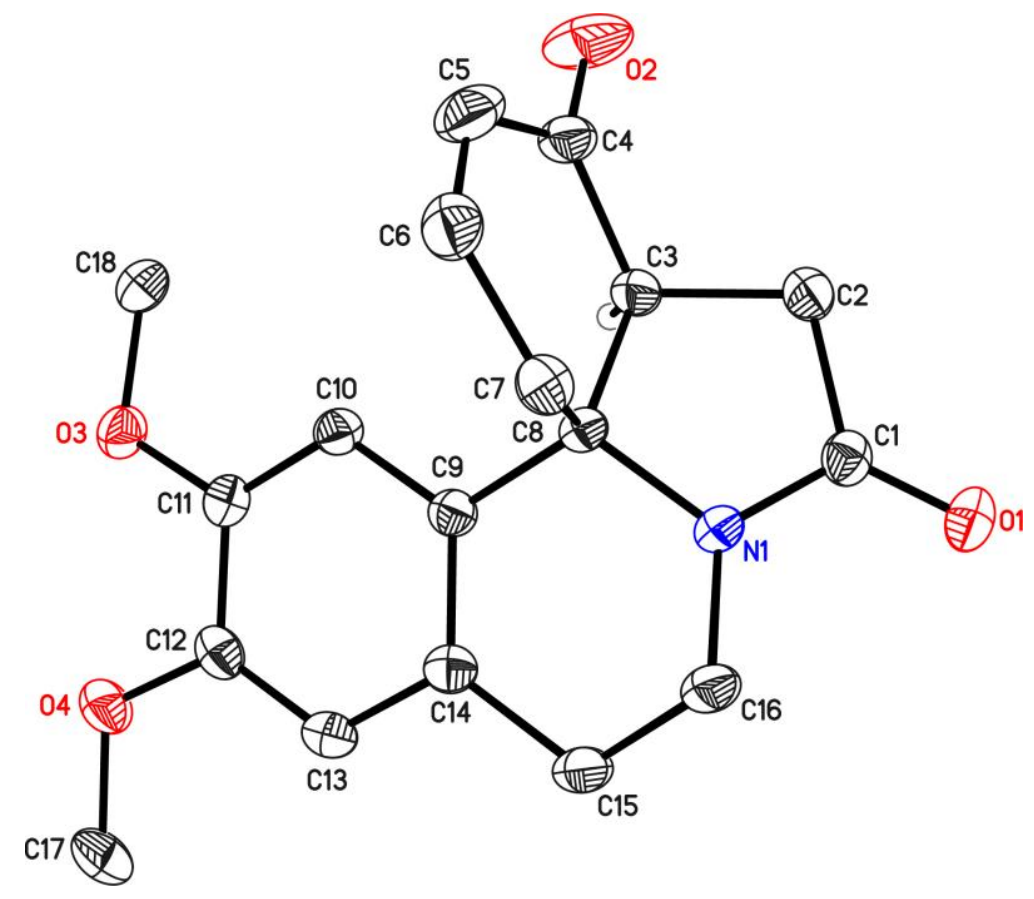

\begin{tabular}{|c|c|c|c|}
\hline Structure code & DK_NTD106c & Z & 2 \\
\hline Empirical formula & $\mathrm{C}_{18} \mathrm{H}_{19} \mathrm{NO}_{4}$ & Crystal size $\left[\mathrm{mm}^{3}\right]$ & $0.18 \times 0.15 \times 0.15$ \\
\hline Formula weight [gmol-1] & 313.34 & $\rho_{\text {calc }}\left[\mathrm{Mgm}^{-3}\right]$ & 1.387 \\
\hline Temperature $[\mathrm{K}]$ & $100(2)$ & $\mu\left[\mathrm{mm}^{-1}\right]$ & 0.098 \\
\hline Wavelength [Å] & 0.71073 & $F(000)$ & 332 \\
\hline Crystal system & Triclinic & $\Theta$ range $\left[{ }^{\circ}\right]$ & 1.88 to 26.73 \\
\hline Space group & $P \overline{1}$ & Reflections collected & 15766 \\
\hline Unit cell dimensions [Å] & & Unique reflections & 3174 \\
\hline$a=$ & $7.9492(7)$ & $R_{\text {int }} / R_{\sigma}$ & $0.0327 / 0.0207$ \\
\hline$b=$ & $8.9757(8)$ & Completeness to $\theta_{\max }$ & $99.6 \%$ \\
\hline$c=$ & $11.3745(10)$ & restraints/parameters & $0 / 210$ \\
\hline$\alpha=$ & $106.9740(10)^{\circ}$ & GooF & 1.036 \\
\hline$\beta=$ & $91.9630(10)^{\circ}$ & $\mathrm{R} 1[\mathrm{l}>2 \sigma(\mathrm{l})]$ & 0.0401 \\
\hline$\gamma=$ & $103.4560(10)^{\circ}$ & wR2 (all data) & 0.1096 \\
\hline Volume $\left[\AA^{3}\right]$ & $750.33(11)$ & diff. peak / hole $\left[\mathrm{e}^{-3}{ }^{-3}\right]$ & $0.323 /-0.195$ \\
\hline Resolution $[\AA ̊]$ & 0.790 & & \\
\hline
\end{tabular}

All hydrogen atoms except $\mathrm{H} 3$ have been omitted. 


\subsection{Structures Measured for Prof. 0 . Wenger}

\subsubsection{Unpublished Structures}

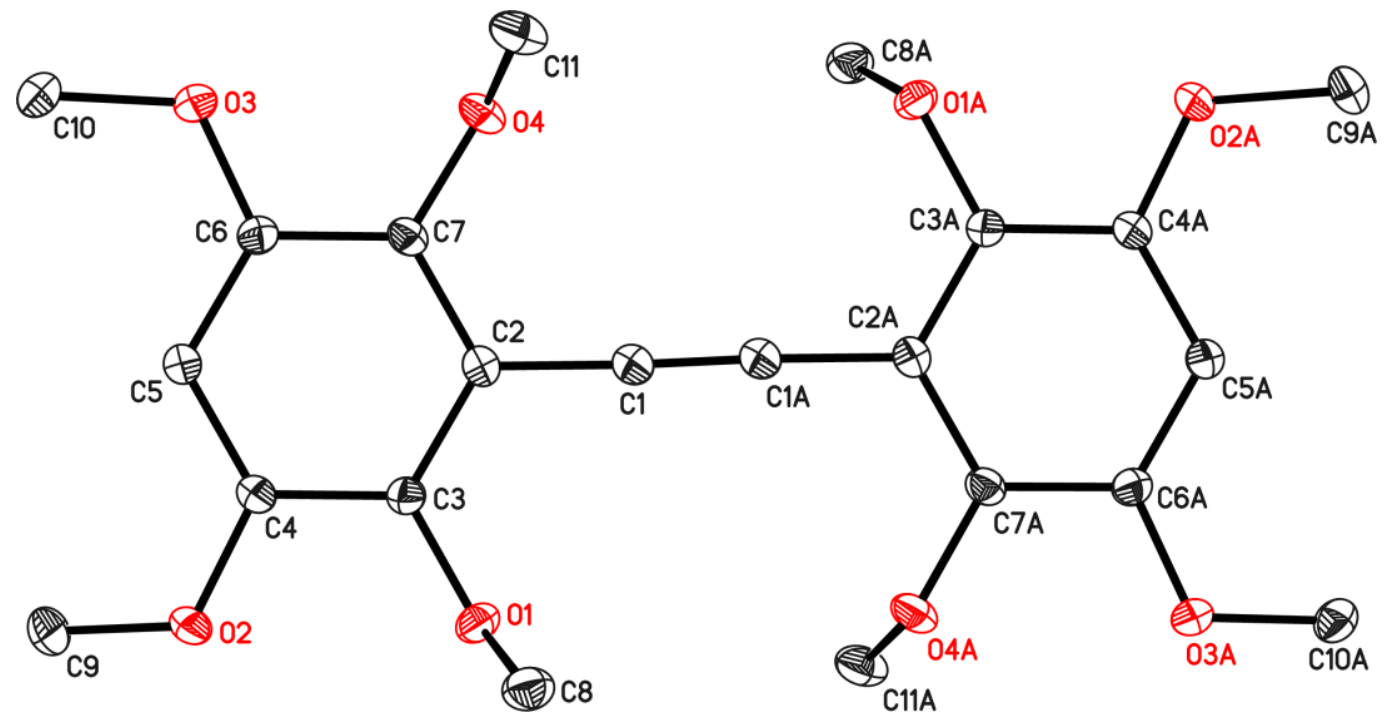

\begin{tabular}{|c|c|c|c|}
\hline Structure code & DK_alkin1 & 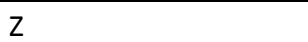 & 2 \\
\hline Empirical formula & $\mathrm{C}_{22} \mathrm{H}_{26} \mathrm{O}_{8}$ & Crystal size $\left[\mathrm{mm}^{3}\right]$ & $0.34 \times 0.20 \times 0.19$ \\
\hline Formula weight [gmol-1] & 418.43 & $\rho_{\text {calc }}\left[\mathrm{Mgm}^{-3}\right]$ & 1.349 \\
\hline Temperature $[\mathrm{K}]$ & $100(2)$ & $\mu\left[\mathrm{mm}^{-1}\right]$ & 0.103 \\
\hline Wavelength $[\AA ̊]$ & 0.71073 & $F(000)$ & 444 \\
\hline Crystal system & Monoclinic & $\Theta$ range $\left[{ }^{\circ}\right]$ & 2.71 to 30.53 \\
\hline Space group & $P 2_{1} / n$ & Reflections collected & 37923 \\
\hline Unit cell dimensions $[\AA ̊]$ & & Unique reflections & 3147 \\
\hline$a=$ & $8.909(3)$ & $R_{\text {int }} / R_{\sigma}$ & $0.0426 / 0.0172$ \\
\hline$b=$ & $9.646(4)$ & Completeness to $\theta_{\max }$ & $99.9 \%$ \\
\hline$c=$ & $12.029(4)$ & restraints/parameters & 35 / 141 \\
\hline$\alpha=$ & $90^{\circ}$ & GooF & 1.088 \\
\hline$\beta=$ & $94.947(9)^{\circ}$ & $\mathrm{R} 1[\mathrm{I}>2 \sigma(\mathrm{I})]$ & 0.0381 \\
\hline$\gamma=$ & $90^{\circ}$ & wR2 (all data) & 0.1062 \\
\hline Volume $\left[\AA^{3}\right]$ & $1029.8(7)$ & diff. peak / hole $\left[e \AA^{-3}\right]$ & 0.509 and -0.226 \\
\hline Resolution $[\AA ̊]$ & 0.700 & & \\
\hline
\end{tabular}




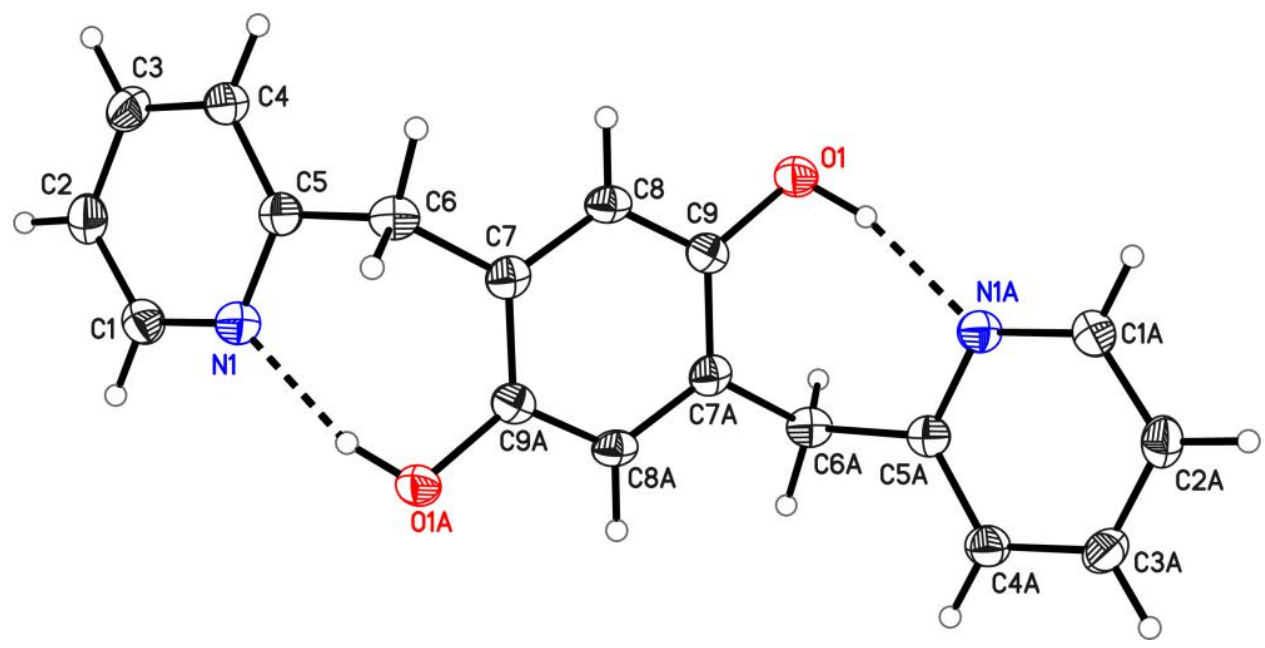

\begin{tabular}{|c|c|c|c|}
\hline Structure code & DK_Bice04=Jorina02 & $Z$ & 2 \\
\hline Empirical formula & $\mathrm{C}_{18} \mathrm{H}_{16} \mathrm{~N}_{2} \mathrm{O}_{2}$ & Crystal size $\left[\mathrm{mm}^{3}\right]$ & $0.11 \times 0.10 \times 0.10$ \\
\hline Formula weight [gmol-1] & 292.33 & $\rho_{\text {calc }}\left[\mathrm{Mgm}^{-3}\right]$ & 1.360 \\
\hline Temperature $[\mathrm{K}]$ & $100(2)$ & $\mu\left[\mathrm{mm}^{-1}\right]$ & 0.090 \\
\hline Wavelength $[\AA ̊]$ & 0.71073 & $F(000)$ & 308 \\
\hline Crystal system & Monoclinic & $\Theta$ range $\left[{ }^{\circ}\right]$ & 2.485 to $25.757^{\circ}$ \\
\hline Space group & $P 2_{1} / n$ & Reflections collected & 15549 \\
\hline Unit cell dimensions $[\AA ̊]$ & & Unique reflections & 1368 \\
\hline$a=$ & $9.2779(11)$ & $\mathrm{R}_{\text {int }} / \mathrm{R}_{\sigma}$ & 0.1277 / 0.0419 \\
\hline$b=$ & $7.9306(9)$ & Completeness to $\theta_{\max }$ & $99.8 \%$ \\
\hline$c=$ & $10.5449(12)$ & restraints/parameters & $0 / 104$ \\
\hline$\alpha=$ & $90^{\circ}$ & GooF & 1.055 \\
\hline$\beta=$ & $113.096(2)^{\circ}$ & $\mathrm{R} 1[\mathrm{I}>2 \sigma(\mathrm{I})]$ & 0.0365 \\
\hline$\gamma=$ & $90^{\circ}$ & wR2 (all data) & 0.0996 \\
\hline Volume $\left[\AA^{3}\right]$ & $713.70(14)$ & diff. peak / hole $\left[\mathrm{e} \AA^{-3}\right]$ & $0.196 /-0.171$ \\
\hline Resolution $[\AA]]$ & 0.818 & & \\
\hline
\end{tabular}




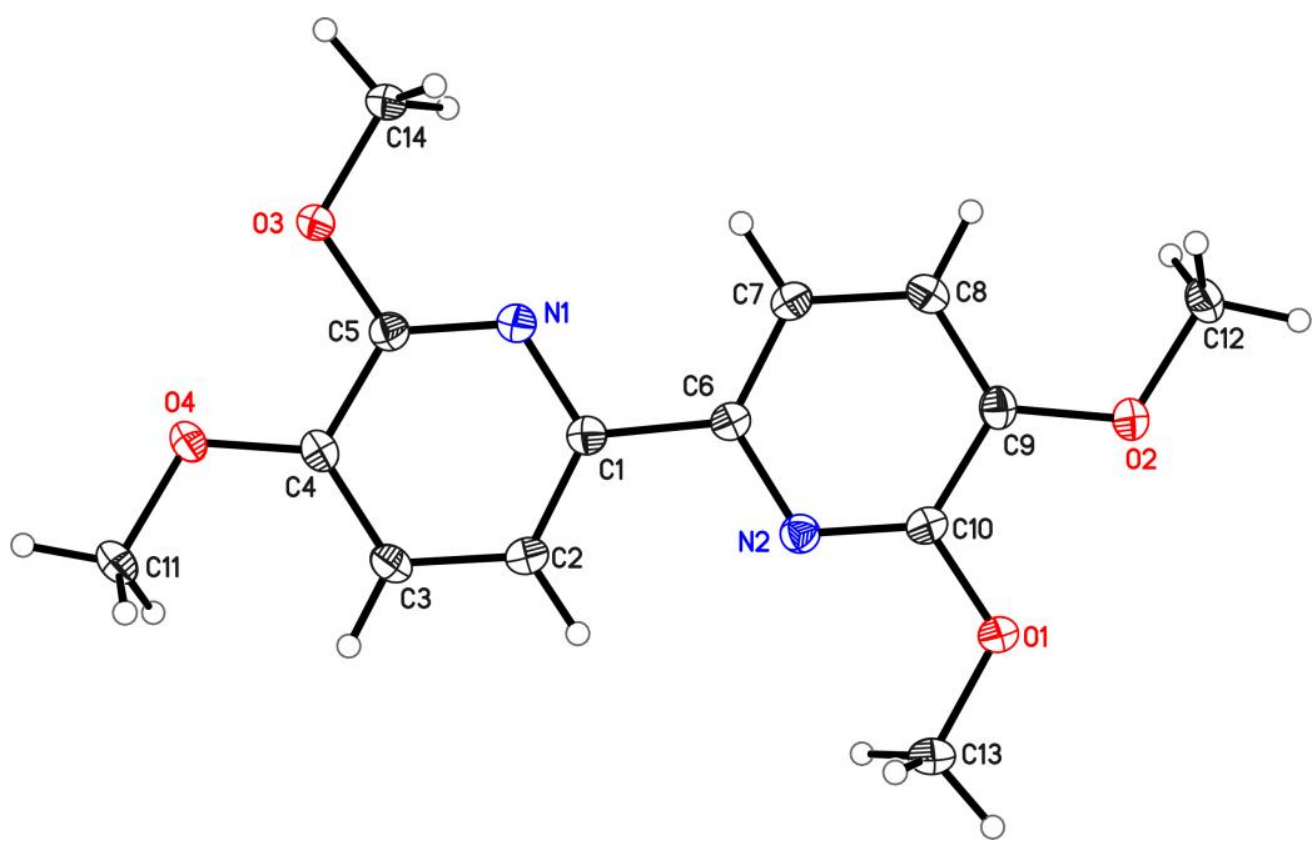

\begin{tabular}{|c|c|c|c|}
\hline Structure code & DK_Bice05 & Z & 8 \\
\hline Empirical formula & $\mathrm{C}_{14} \mathrm{H}_{16} \mathrm{~N}_{2} \mathrm{O}_{4}$ & Crystal size $\left[\mathrm{mm}^{3}\right]$ & $0.10 \times 0.10 \times 0.10$ \\
\hline Formula weight [gmol-1] & 276.29 & $\rho_{\text {calc }}\left[\mathrm{Mgm}^{-3}\right]$ & 1.387 \\
\hline Temperature $[\mathrm{K}]$ & $100(2)$ & $\mu\left[\mathrm{mm}^{-1}\right]$ & 0.103 \\
\hline Wavelength $[\AA ̊]$ & 0.71073 & $F(000)$ & 1168 \\
\hline Crystal system & Orthorhombic & $\Theta$ range $\left[{ }^{\circ}\right]$ & 1.78 to 27.19 \\
\hline Space group & Pbca & Reflections collected & 62442 \\
\hline Unit cell dimensions $[\AA ̊]$ & & Unique reflections & 2942 \\
\hline$a$ & $8.7682(7)$ & $\mathrm{R}_{\text {int }} / \mathrm{R}_{\sigma}$ & 0.0619 / 0.0194 \\
\hline$b$ & $13.1706(11)$ & Completeness to $\theta_{\max }$ & $100.0 \%$ \\
\hline$c$ & $22.9077(19)$ & restraints/parameters & $0 / 181$ \\
\hline$\alpha$ & $90^{\circ}$ & GooF & 1.037 \\
\hline$\beta$ & $90^{\circ}$ & $\mathrm{R} 1[\mathrm{I}>2 \sigma(\mathrm{I})]$ & 0.0381 \\
\hline$\gamma$ & $90^{\circ}$ & wR2 (all data) & 0.1013 \\
\hline Volume $\left[\AA^{3}\right]$ & $2645.4(4)$ & diff. peak / hole $\left[\mathrm{e} \AA^{-3}\right]$ & $0.252 /-0.252$ \\
\hline Resolution $[\AA ̊]$ & 0.778 & & \\
\hline
\end{tabular}




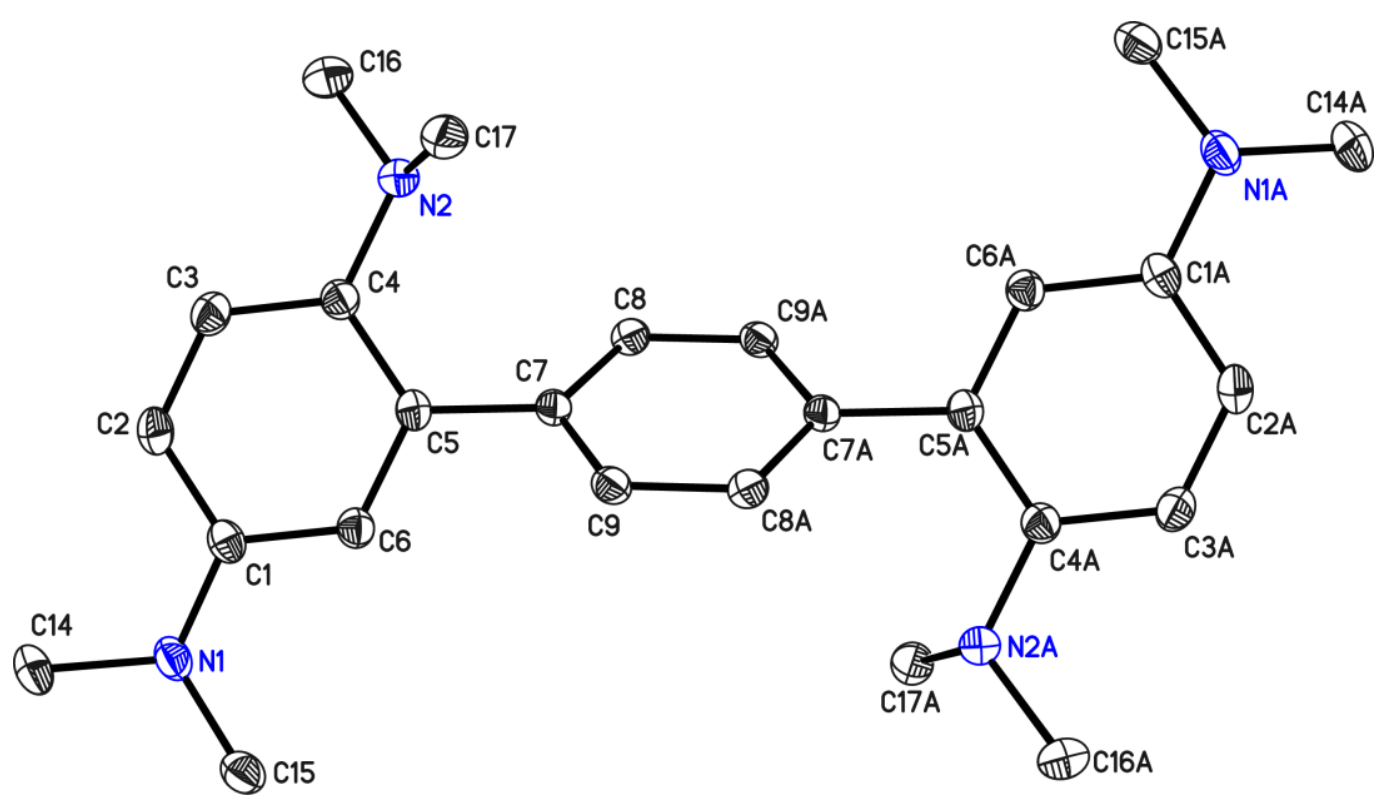

\begin{tabular}{|c|c|c|c|}
\hline Structure code & DK_Bice06 & Z & 2 \\
\hline Empirical formula & $\mathrm{C}_{26} \mathrm{H}_{34} \mathrm{~N}_{4}$ & Crystal size $\left[\mathrm{mm}^{3}\right]$ & $0.2 \times 0.2 \times 0.1$ \\
\hline Formula weight [gmol-1] & 402.57 & $\rho_{\text {calc }}\left[\mathrm{Mgm}^{-3}\right]$ & 1.184 \\
\hline Temperature $[\mathrm{K}]$ & $100(2)$ & $\mu\left[\mathrm{mm}^{-1}\right]$ & 0.071 \\
\hline Wavelength $[\AA ̊]$ & 0.71073 & $F(000)$ & 436 \\
\hline Crystal system & Monoclinic & $\Theta$ range $\left[{ }^{\circ}\right]$ & 2.24 to 27.62 \\
\hline Space group & $P 2_{1} / c$ & Reflections collected & 28608 \\
\hline Unit cell dimensions $[\AA ̊]$ & & Unique reflections & 2622 \\
\hline$a=$ & $8.5600(14)$ & $R_{\text {int }} / R_{\sigma}$ & $0.0327 / 0.0142$ \\
\hline$b=$ & $7.2535(12)$ & Completeness to $\theta_{\max }$ & $99.8 \%$ \\
\hline$c=$ & $18.413(3)$ & restraints/parameters & $0 / 140$ \\
\hline$\alpha=$ & $90^{\circ}$ & GooF & 1.043 \\
\hline$\beta=$ & $99.093(2)^{\circ}$ & $\mathrm{R} 1[\mathrm{I}>2 \sigma(\mathrm{I})]$ & 0.0401 \\
\hline$\gamma=$ & $90^{\circ}$ & wR2 (all data) & 0.1117 \\
\hline Volume $\left[\AA^{3}\right]$ & $1128.9(3)$ & diff. peak / hole $\left[\mathrm{e}^{-3}\right]$ & $0.316 /-0.209$ \\
\hline Resolution $[\AA ̊]$ & 0.767 & & \\
\hline
\end{tabular}




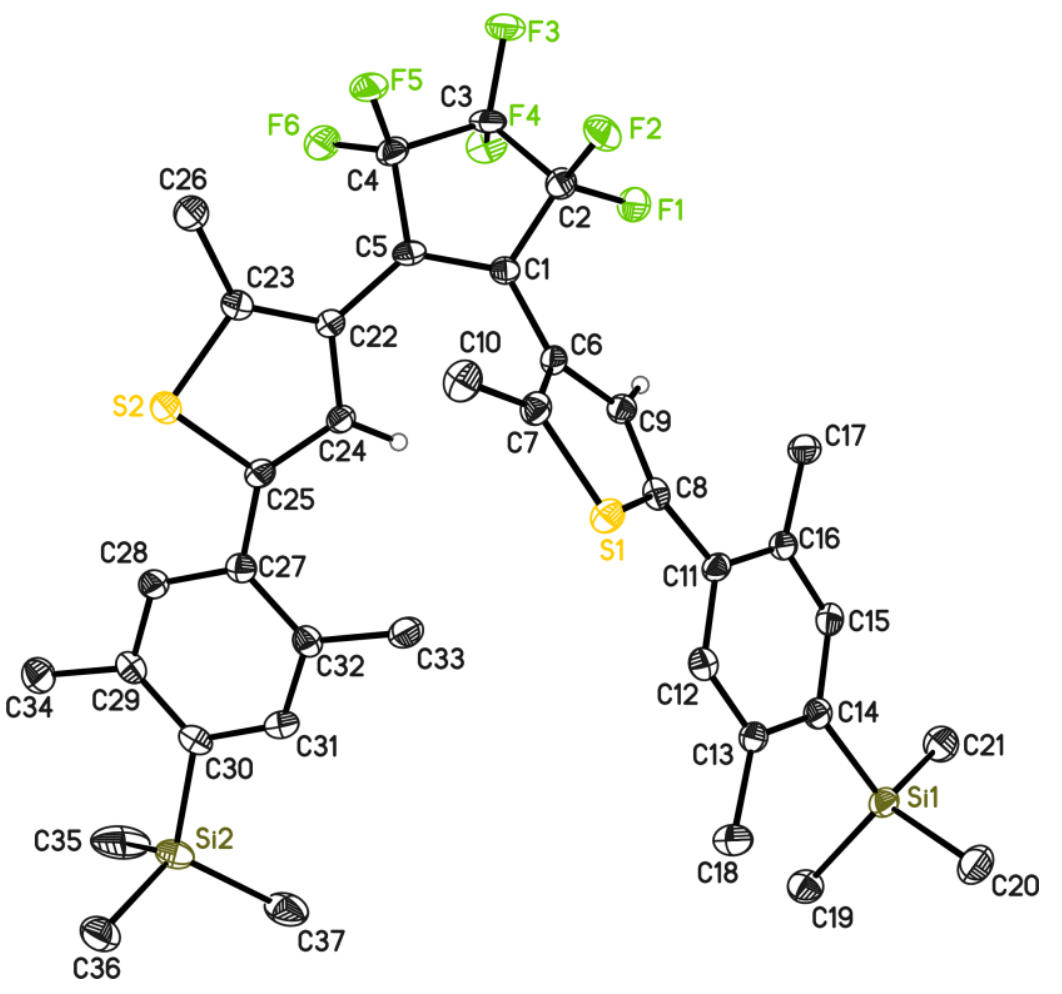

\begin{tabular}{|c|c|c|c|}
\hline Structure code & DK_HB10029 & Z & $7316.1(10)$ \\
\hline Empirical formula & $\mathrm{C}_{37} \mathrm{H}_{42} \mathrm{~F}_{6} \mathrm{~S}_{2} \mathrm{Si}_{2}$ & Crystal size $\left[\mathrm{mm}^{3}\right]$ & $0.08 \times 0.08 \times 0.05$ \\
\hline Formula weight [gmol-1] & 721.01 & $\rho_{\text {calc }}\left[\mathrm{Mgm}^{-3}\right]$ & 1.309 \\
\hline Temperature $[\mathrm{K}]$ & $100(2)$ & $\mu\left[\mathrm{mm}^{-1}\right]$ & 0.267 \\
\hline Wavelength $[\AA ̊]$ & 0.71073 & $F(000)$ & 3024 \\
\hline Crystal system & Orthorhombic & $\Theta$ range $\left[{ }^{\circ}\right]$ & 1.17 to 25.39 \\
\hline Space group & Pbca & Reflections collected & 76146 \\
\hline Unit cell dimensions $[\AA ̊]$ & & Unique reflections & 6722 \\
\hline$a=$ & $7.7561(6)$ & $\mathrm{R}_{\text {int }} / \mathrm{R}_{\sigma}$ & $0.0890 / 0.0392$ \\
\hline$b=$ & $27.164(2)$ & Completeness to $\theta_{\max }$ & $99.9 \%$ \\
\hline$c=$ & $34.725(3)$ & restraints/parameters & $0 / 436$ \\
\hline$\alpha=$ & $90^{\circ}$ & GooF & 1.020 \\
\hline$\beta=$ & $90^{\circ}$ & $\mathrm{R} 1[\mathrm{I}>2 \sigma(\mathrm{I})]$ & 0.0387 \\
\hline$\gamma=$ & $90^{\circ}$ & wR2 (all data) & 0.0891 \\
\hline Volume $\left[\AA^{3}\right]$ & $7316.1(10)$ & diff. peak / hole $\left[\mathrm{e} \AA^{-3}\right]$ & $0.314 /-0.279$ \\
\hline Resolution $[\AA ̊]$ & 0.829 & & \\
\hline
\end{tabular}




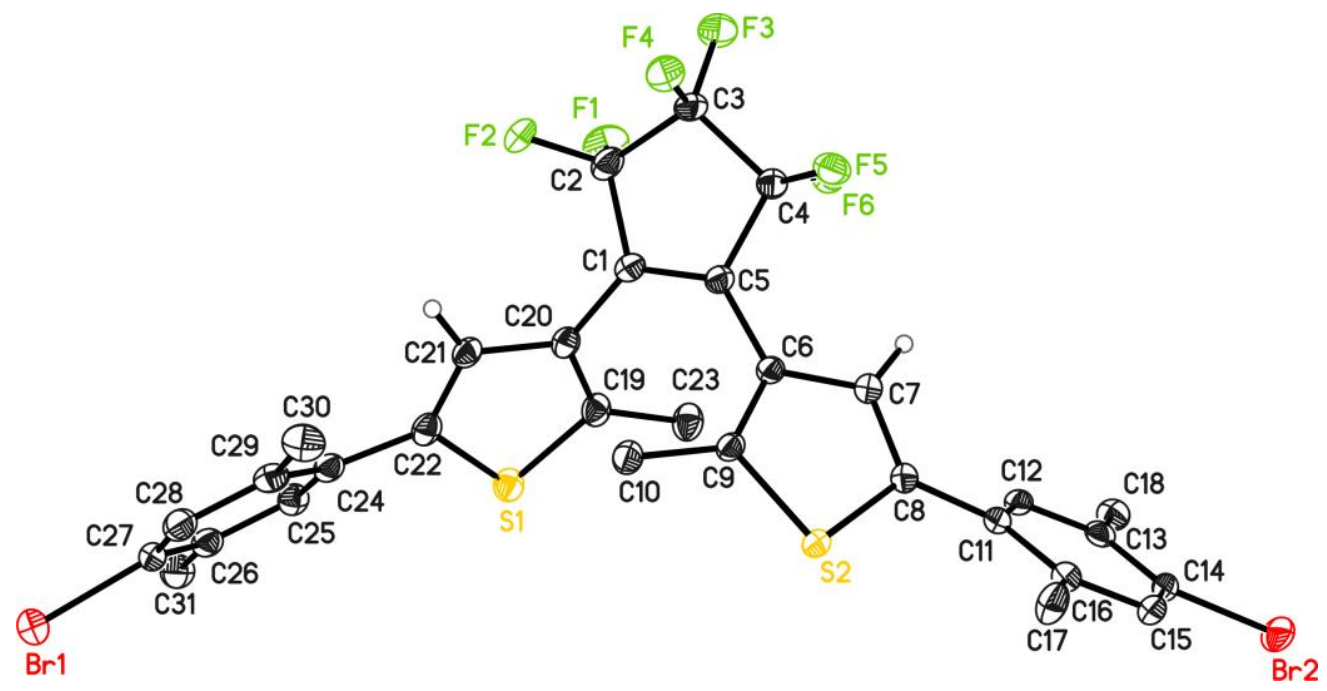

\begin{tabular}{|c|c|c|c|}
\hline Structure code & DK_HB10032 & z & 2 \\
\hline Empirical formula & $\mathrm{C}_{31} \mathrm{H}_{24} \mathrm{Br}_{2} \mathrm{~F}_{6} \mathrm{~S}_{2}$ & Crystal size $\left[\mathrm{mm}^{3}\right]$ & $0.14 \times 0.14 \times 0.11$ \\
\hline Formula weight [gmol-1] & 734.44 & $\rho_{\text {calc }}\left[\mathrm{Mgm}^{-3}\right]$ & 1.692 \\
\hline Temperature [K] & $100(2)$ & $\mu\left[\mathrm{mm}^{-1}\right]$ & 3.016 \\
\hline Wavelength [Å] & 0.71073 & $F(000)$ & 732 \\
\hline Crystal system & Triclinic & $\Theta$ range $\left[{ }^{\circ}\right]$ & 1.47 to 27.60 \\
\hline Space group & $P \overline{1}$ & Reflections collected & 22912 \\
\hline Unit cell dimensions [Å] & & Unique reflections & 6697 \\
\hline$a=$ & $8.7376(10)$ & $R_{\text {int }} / R_{\sigma}$ & $0.0278 / 0.0278$ \\
\hline$b=$ & $11.9683(15)$ & Completeness to $\theta_{\max }$ & $99.7 \%$ \\
\hline$c=$ & $14.0773(16)$ & restraints/parameters & $0 / 376$ \\
\hline$\alpha=$ & $87.869(3)^{\circ}$ & GooF & 1.041 \\
\hline$\beta=$ & $79.123(2)^{\circ}$ & $\mathrm{R} 1[\mathrm{l}>2 \sigma(\mathrm{I})]$ & 0.0300 \\
\hline$\gamma=$ & $86.105(3)^{\circ}$ & wR2 (all data) & 0.0759 \\
\hline Volume $\left[\AA^{3}\right]$ & $1441.9(3)$ & diff. peak / hole $\left[\mathrm{e}^{-3}\right]$ & $0.646 /-0.608$ \\
\hline Resolution [Å] & 0.767 & & \\
\hline
\end{tabular}




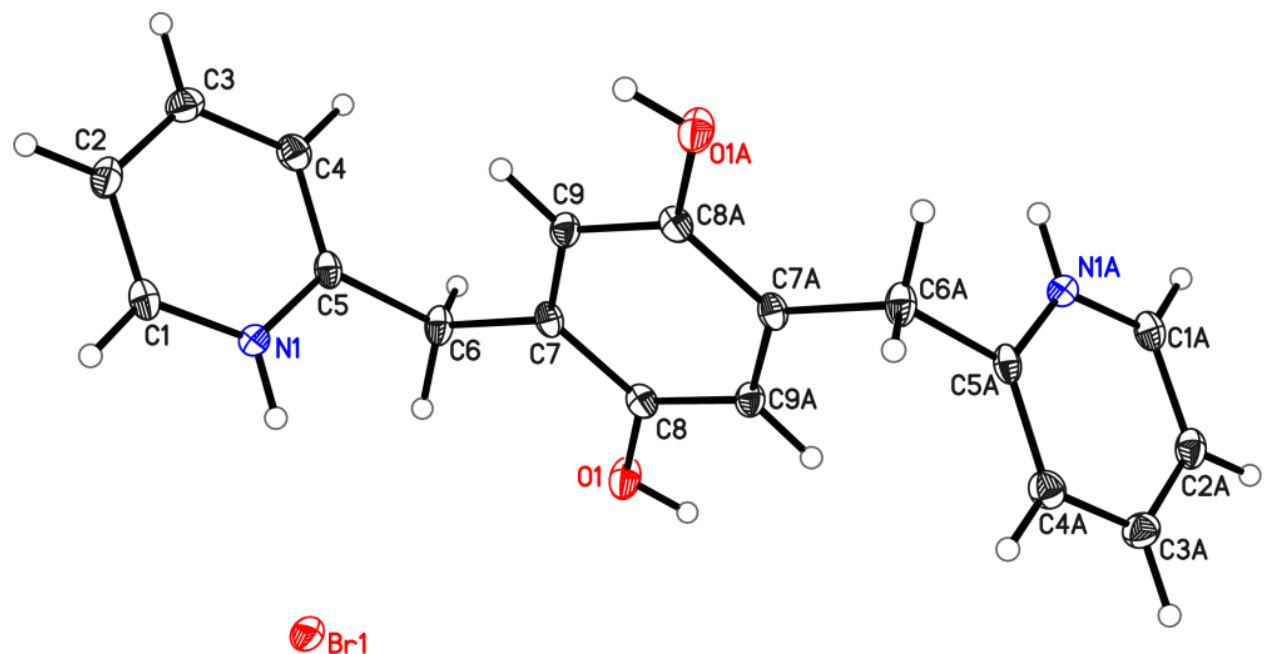

\begin{tabular}{|c|c|c|c|}
\hline Structure code & DK_Jorina1 & Z & 2 \\
\hline Empirical formula & $\mathrm{C}_{18} \mathrm{H}_{18} \mathrm{Br}_{2} \mathrm{~N}_{2} \mathrm{O}_{2}$ & Crystal size $\left[\mathrm{mm}^{3}\right]$ & $0.24 \times 0.18 \times 0.17$ \\
\hline Formula weight [gmol-1] & 454.16 & $\rho_{\text {calc }}\left[\mathrm{Mgm}^{-3}\right]$ & 1.748 \\
\hline Temperature $[\mathrm{K}]$ & $100(2)$ & $\mu\left[\mathrm{mm}^{-1}\right]$ & 4.710 \\
\hline Wavelength $[\AA ̊]$ & 0.71073 & $F(000)$ & 452 \\
\hline Crystal system & Monoclinic & $\Theta$ range $\left[{ }^{\circ}\right]$ & 2.18 to 27.52 \\
\hline Space group & $P 2_{1} / c$ & Reflections collected & 21975 \\
\hline Unit cell dimensions $[\AA ̊]$ & & Unique reflections & 1993 \\
\hline$a=$ & $4.9232(9)$ & $R_{\text {int }} / R_{\sigma}$ & $0.0275 / 0.0124$ \\
\hline$b=$ & $12.558(2)$ & Completeness to $\theta_{\max }$ & $100.0 \%$ \\
\hline$c=$ & $14.055(3)$ & restraints/parameters & $0 / 110$ \\
\hline$\alpha=$ & $90^{\circ}$ & GooF & 1.100 \\
\hline$\beta=$ & $96.763(3)^{\circ}$ & $\mathrm{R} 1[\mathrm{I}>2 \sigma(\mathrm{I})]$ & 0.0166 \\
\hline$\gamma=$ & $90^{\circ}$ & wR2 (all data) & 0.0397 \\
\hline Volume $\left[\AA^{3}\right]$ & $862.9(3)$ & diff. peak / hole $\left[\mathrm{e} \AA^{-3}\right]$ & $0.344 /-0.268$ \\
\hline Resolution $[\AA ̊]$ & 0.769 & & \\
\hline
\end{tabular}




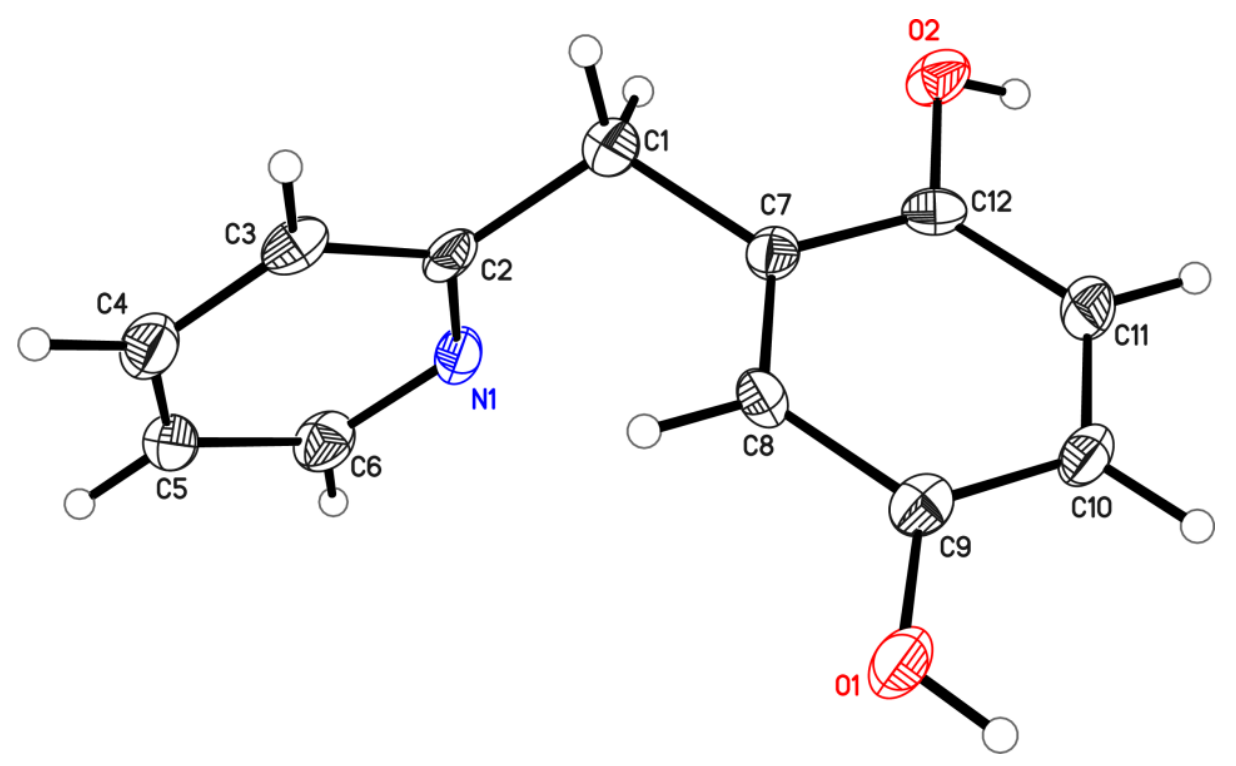

\begin{tabular}{|c|c|c|c|}
\hline Structure code & DK_Jorina03 & z & 8 \\
\hline Empirical formula & $\mathrm{C}_{12} \mathrm{H}_{11} \mathrm{NO}_{2}$ & Crystal size $\left[\mathrm{mm}^{3}\right]$ & $0.2 \times 0.2 \times 0.1$ \\
\hline Formula weight [gmol-1] & 201.22 & $\rho_{\text {calc }}\left[\mathrm{Mgm}^{-3}\right]$ & 1.375 \\
\hline Temperature $[\mathrm{K}]$ & $100(2)$ & $\mu\left[\mathrm{mm}^{-1}\right]$ & 0.094 \\
\hline Wavelength [Å] & 0.71073 & $F(000)$ & 848 \\
\hline Crystal system & Monoclinic & $\Theta$ range $\left[{ }^{\circ}\right]$ & 2.36 to 25.35 \\
\hline Space group & $C 2 / c$ & Reflections collected & 4113 \\
\hline Unit cell dimensions [Å] & & Unique reflections & 1778 \\
\hline$a=$ & $17.601(14)$ & $R_{\text {int }} / R_{\sigma}$ & $0.0946 / 0.1856$ \\
\hline$b=$ & $7.705(7)$ & Completeness to $\theta_{\max }$ & $99.3 \%$ \\
\hline$c=$ & $14.591(13)$ & restraints/parameters & $0 / 138$ \\
\hline$\alpha=$ & $90^{\circ}$ & GooF & 0.709 \\
\hline$\beta=$ & $100.67(2)^{\circ}$ & $\mathrm{R} 1[\mathrm{I}>2 \sigma(\mathrm{l})]$ & 0.0483 \\
\hline$\gamma=$ & $90^{\circ}$. & wR2 (all data) & 0.0739 \\
\hline Volume $\left[\AA^{3}\right]$ & 1945(3) & diff. peak / hole $\left[\AA^{-3}\right]$ & $0.312 /-0.210$ \\
\hline Resolution $[\AA ̊]$ & 0.830 & & \\
\hline
\end{tabular}




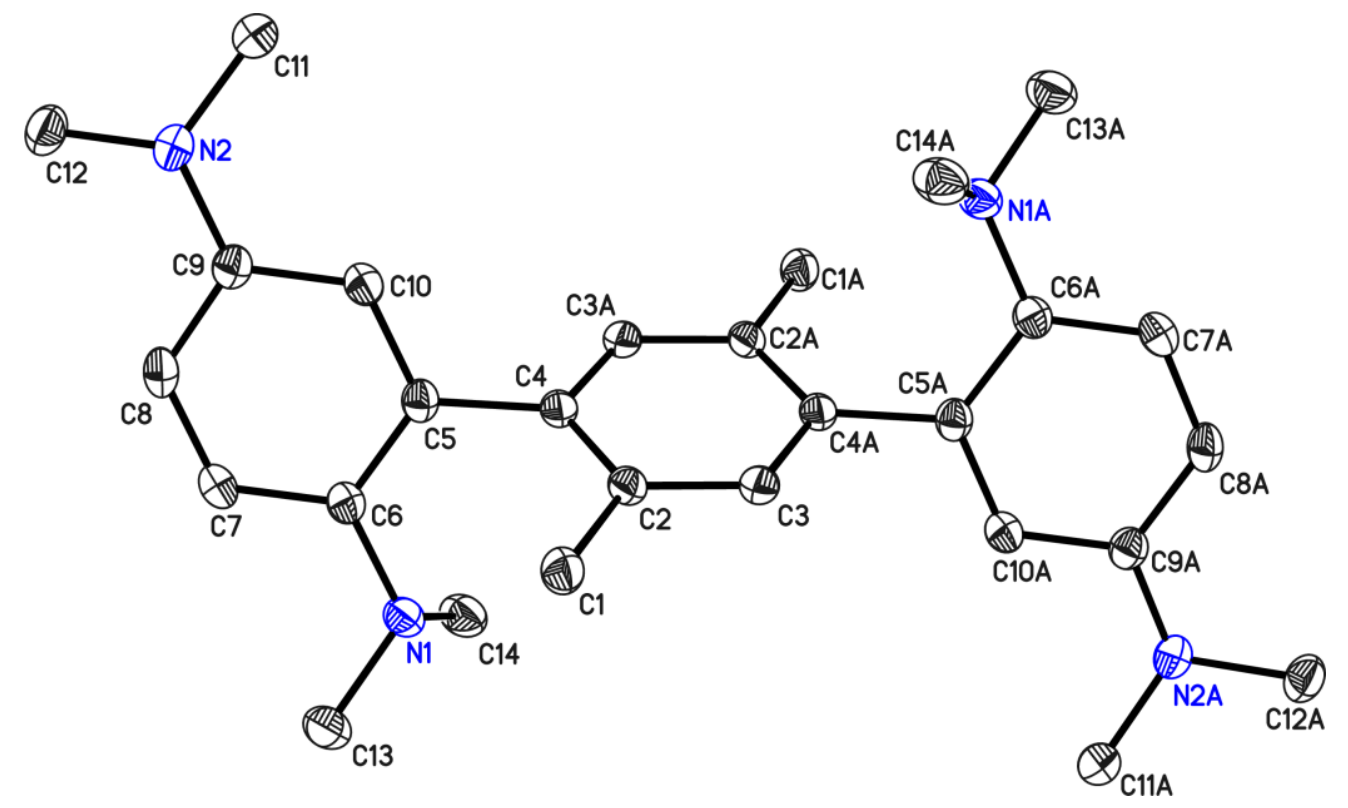

\begin{tabular}{|c|c|c|c|}
\hline Structure code & DK_Julia1 & $\mathrm{Z}$ & 2 \\
\hline Empirical formula & $\mathrm{C}_{28} \mathrm{H}_{38} \mathrm{~N}_{4}$ & Crystal size $\left[\mathrm{mm}^{3}\right]$ & $0.1 \times 0.09 \times 0.05$ \\
\hline Formula weight [gmol-1] & 430.62 & $\rho_{\text {calc }}\left[\mathrm{Mgm}^{-3}\right]$ & 1.206 \\
\hline Temperature $[\mathrm{K}]$ & $100(2)$ & $\mu\left[\mathrm{mm}^{-1}\right]$ & 0.072 \\
\hline Wavelength $[\AA]$ & 0.71073 & $F(000)$ & 468 \\
\hline Crystal system & Monoclinic & $\Theta$ range $\left[{ }^{\circ}\right]$ & 2.19 to 25.37 \\
\hline Space group & $P 2_{1} / n$ & Reflections collected & 25041 \\
\hline Unit cell dimensions $[\AA ̊]$ & & Unique reflections & 2173 \\
\hline$a$ & $8.4177(17)$ & $R_{\text {int }} / R_{\sigma}$ & $0.0689 / 0.0308$ \\
\hline$b$ & $7.5768(15)$ & Completeness to $\theta_{\max }$ & $99.9 \%$ \\
\hline$c$ & $19.032(4)$ & restraints/parameters & $0 / 150$ \\
\hline$\alpha$ & $90^{\circ}$ & GooF & 1.054 \\
\hline$\beta$ & $102.408(3)^{\circ}$ & $\mathrm{R} 1[\mathrm{I}>2 \sigma(\mathrm{I})]$ & 0.0481 \\
\hline$\gamma$ & $90^{\circ}$ & wR2 (all data) & 0.1334 \\
\hline Volume $\left[\AA^{3}\right]$ & $1185.5(4)$ & diff. peak / hole $\left[\mathrm{e} \AA^{-3}\right]$ & $0.189 /-0.255$ \\
\hline Resolution $[\AA]]$ & 0.829 & & \\
\hline
\end{tabular}




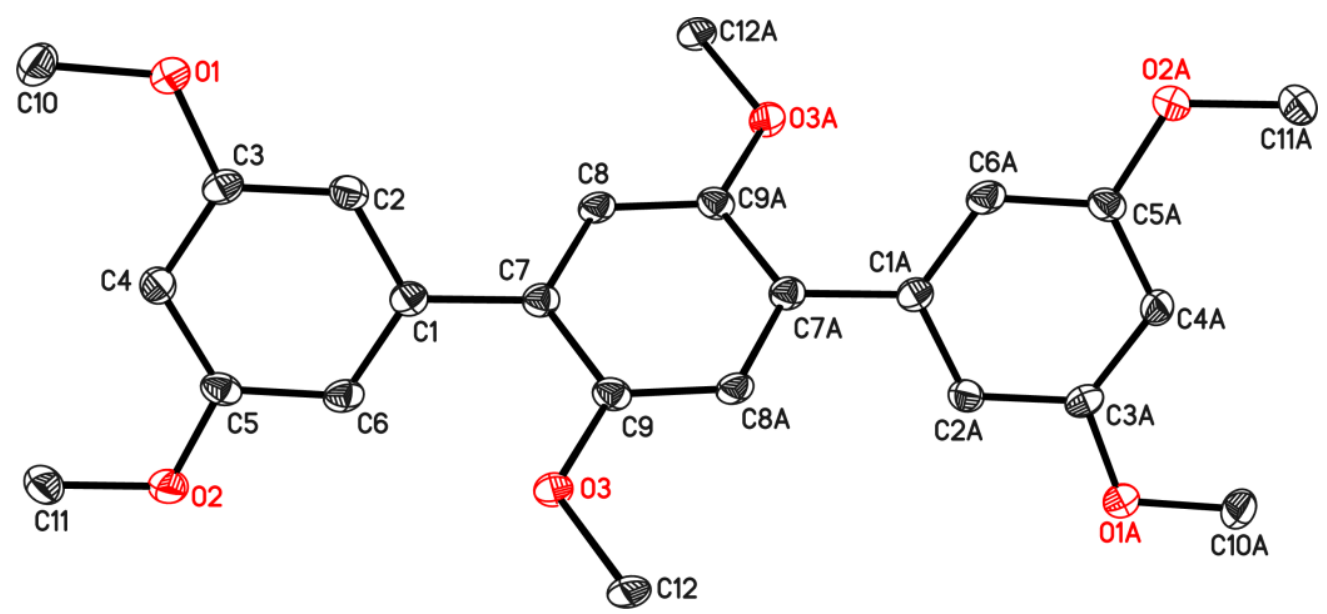

\begin{tabular}{|c|c|c|c|}
\hline Structure code & DK_PhOMeKette & Z & 4 \\
\hline Empirical formula & $\mathrm{C}_{24} \mathrm{H}_{26} \mathrm{O}_{6}$ & Crystal size $\left[\mathrm{mm}^{3}\right]$ & $0.18 \times 0.09 \times 0.03$ \\
\hline Formula weight [gmol-1] & 410.45 & $\rho_{\text {calc }}\left[\mathrm{Mgm}^{-3}\right]$ & 1.328 \\
\hline Temperature $[\mathrm{K}]$ & $100(1)$ & $\mu\left[\mathrm{mm}^{-1}\right]$ & 0.095 \\
\hline Wavelength $[\AA ̊]$ & 0.71073 & $F(000)$ & 872 \\
\hline Crystal system & Orthorombic & $\Theta$ range $\left[{ }^{\circ}\right]$ & 2.27 to 26.03 \\
\hline Space group & Pbca & Reflections collected & 58978 \\
\hline Unit cell dimensions $[\AA ̊]$ & & Unique reflections & 2022 \\
\hline$a$ & $12.5993(14)$ & $R_{\text {int }} / R_{\sigma}$ & 0.0742 / 0.0183 \\
\hline$b$ & $9.0968(9)$ & Completeness to $\theta_{\max }$ & $100.0 \%$ \\
\hline$c$ & $17.917(2)$ & restraints/parameters & $0 / 139$ \\
\hline$\alpha$ & $90^{\circ}$ & GooF & 1.080 \\
\hline$\beta$ & $90^{\circ}$ & $\mathrm{R} 1[\mathrm{I}>2 \sigma(\mathrm{I})]$ & 0.0410 \\
\hline$\gamma$ & $90^{\circ}$ & wR2 (all data) & 0.1046 \\
\hline Volume $\left[\AA^{3}\right]$ & $2053.5(4)$ & diff. peak / hole $\left[\mathrm{e}^{-3}\right]$ & $0.233 /-0.238$ \\
\hline Resolution $[\AA ̊]$ & 0.810 & & \\
\hline
\end{tabular}




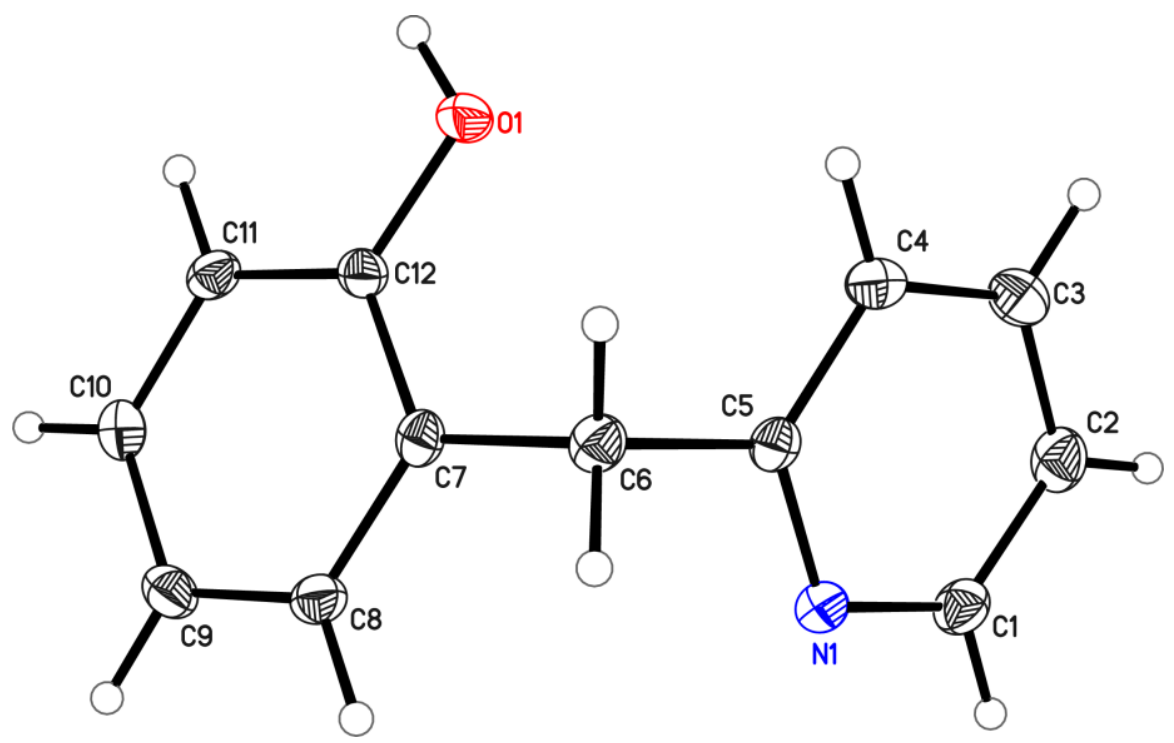

\begin{tabular}{|c|c|c|c|}
\hline Structure code & DK_PHOhPy01 887369 & Z & 8 \\
\hline Empirical formula & $\mathrm{C}_{12} \mathrm{H}_{11} \mathrm{NO}$ & Crystal size $\left[\mathrm{mm}^{3}\right]$ & $0.1 \times 0.1 \times 0.01$ \\
\hline Formula weight [gmol-1] & 185.22 & $\rho_{\text {calc }}\left[\mathrm{Mgm}^{-3}\right]$ & 1.286 \\
\hline Temperature $[\mathrm{K}]$ & $100(2)$ & $\mu\left[\mathrm{mm}^{-1}\right]$ & 0.082 \\
\hline Wavelength $[\AA ̊]$ & 0.71073 & $F(000)$ & 784 \\
\hline Crystal system & Monoclinic & $\Theta$ range $\left[{ }^{\circ}\right]$ & 2.57 to 26.37 \\
\hline Space group & $C 2 / c$ & Reflections collected & 21671 \\
\hline Unit cell dimensions $[\AA ̊]$ & & Unique reflections & 1955 \\
\hline$a=$ & $10.853(5)$ & $\mathrm{R}_{\text {int }} / \mathrm{R}_{\sigma}$ & $0.0536 / 0.0284$ \\
\hline$b=$ & $11.672(3)$ & Completeness to $\theta_{\max }$ & $100.0 \%$ \\
\hline$c=$ & $15.164(6)$ & restraints/parameters & $1 / 134$ \\
\hline$\alpha=$ & $90^{\circ}$ & GooF & 1.061 \\
\hline$\beta=$ & $94.857(12)^{\circ}$ & $\mathrm{R} 1[\mathrm{I}>2 \sigma(\mathrm{I})]$ & 0.0439 \\
\hline$\gamma=$ & $90^{\circ}$ & wR2 (all data) & 0.0970 \\
\hline Volume $\left[\AA^{3}\right]$ & 1913.9(13) & diff. peak / hole $\left[\mathrm{e} \AA^{-3}\right]$ & $0.249 /-0.213$ \\
\hline Resolution $[\AA]$ & 0.800 & & \\
\hline
\end{tabular}




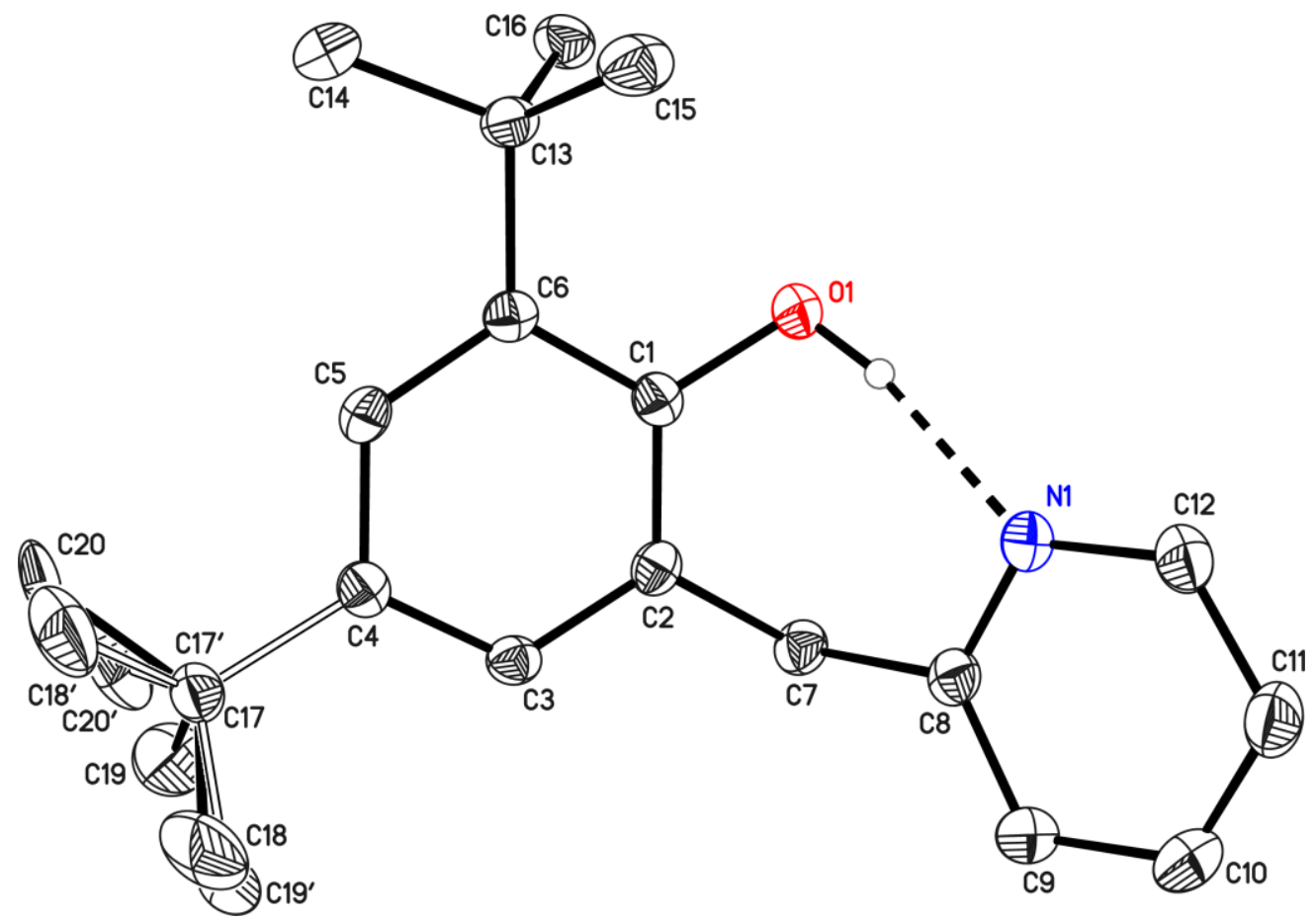

\begin{tabular}{|c|c|c|c|}
\hline Structure code & DK_SL02 887370 & Z & 4 \\
\hline Empirical formula & $\mathrm{C}_{20} \mathrm{H}_{27} \mathrm{NO}$ & Crystal size $\left[\mathrm{mm}^{3}\right]$ & $0.3 \times 0.2 \times 0.18$ \\
\hline Formula weight [gmol-1] & 297.43 & $\rho_{\text {calc }}\left[\mathrm{Mgm}^{-3}\right]$ & 1.120 \\
\hline Temperature $[\mathrm{K}]$ & $100(2)$ & $\mu\left[\mathrm{mm}^{-1}\right]$ & 0.068 \\
\hline Wavelength $[\AA ̊]$ & 0.71073 & $F(000)$ & 648 \\
\hline Crystal system & Monoclinic & $\Theta$ range $\left[{ }^{\circ}\right]$ & 2.30 to 26.77 \\
\hline Space group & $P 2_{1} / n$ & Reflections collected & 35062 \\
\hline Unit cell dimensions $[\AA ̊]$ & & Unique reflections & 3744 \\
\hline$a=$ & $10.120(3)$ & $\mathrm{R}_{\text {int }} / \mathrm{R}_{\sigma}$ & $0.0289 / 0.0149$ \\
\hline$b=$ & $16.751(5)$ & Completeness to $\theta_{\max }$ & $99.8 \%$ \\
\hline$c=$ & $11.023(3)$ & restraints/parameters & $154 / 234$ \\
\hline$\alpha=$ & $90^{\circ}$ & GooF & 1.020 \\
\hline$\beta=$ & $109.267(9)^{\circ}$ & $\mathrm{R} 1[\mathrm{I}>2 \sigma(\mathrm{I})]$ & 0.0432 \\
\hline$\gamma=$ & $90^{\circ}$ & wR2 (all data) & 0.1126 \\
\hline Volume $\left[\AA^{3}\right]$ & $1763.8(9)$ & diff. peak / hole $\left[\mathrm{e} \AA^{-3}\right]$ & $0.298 /-0.184$ \\
\hline Resolution $[\AA]]$ & 0.789 & & \\
\hline
\end{tabular}




\subsection{Structures Measured for Prof. D. B. Werz}

\subsubsection{Published Structures}

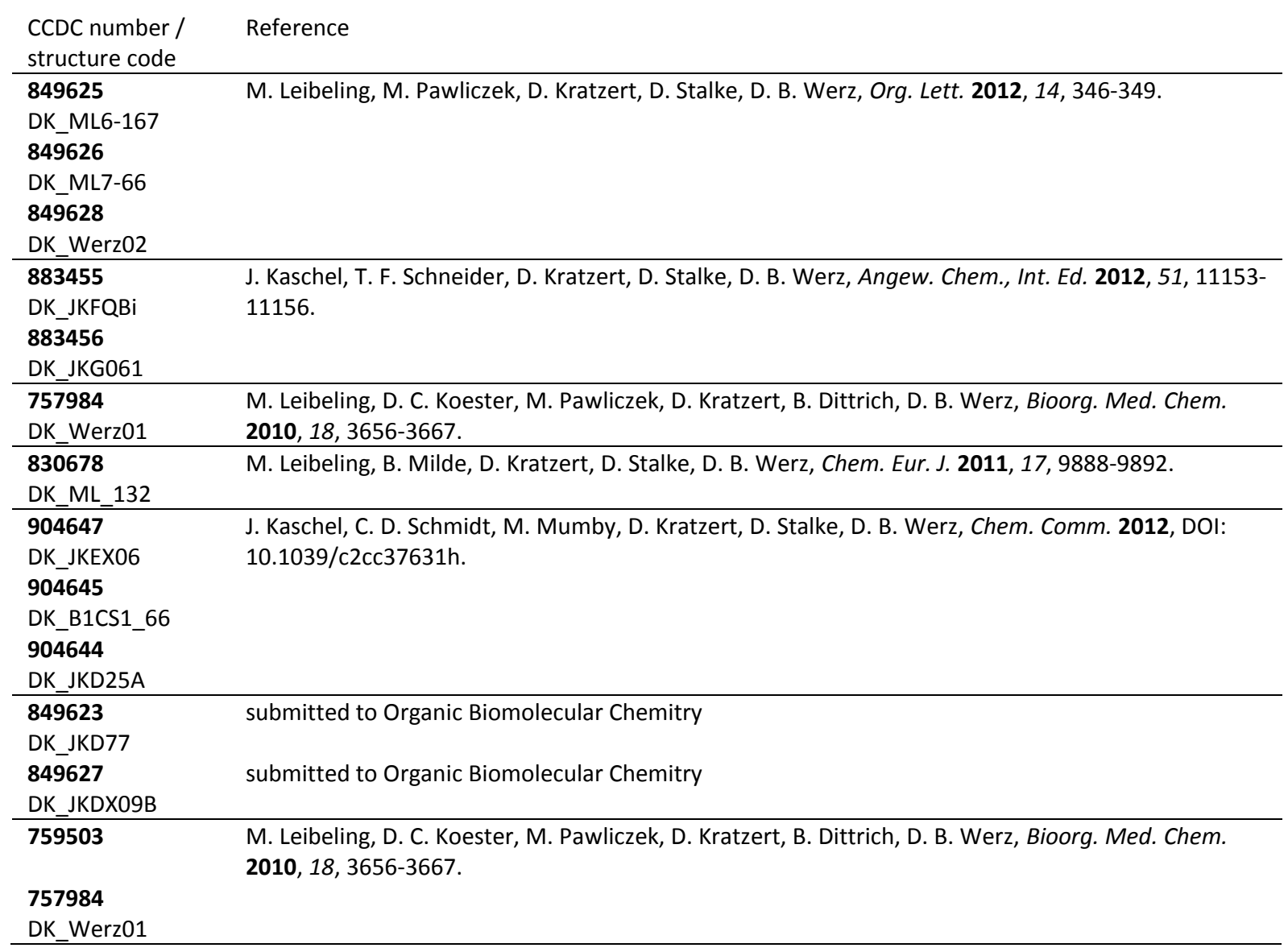




\subsubsection{Unpublished Structures}

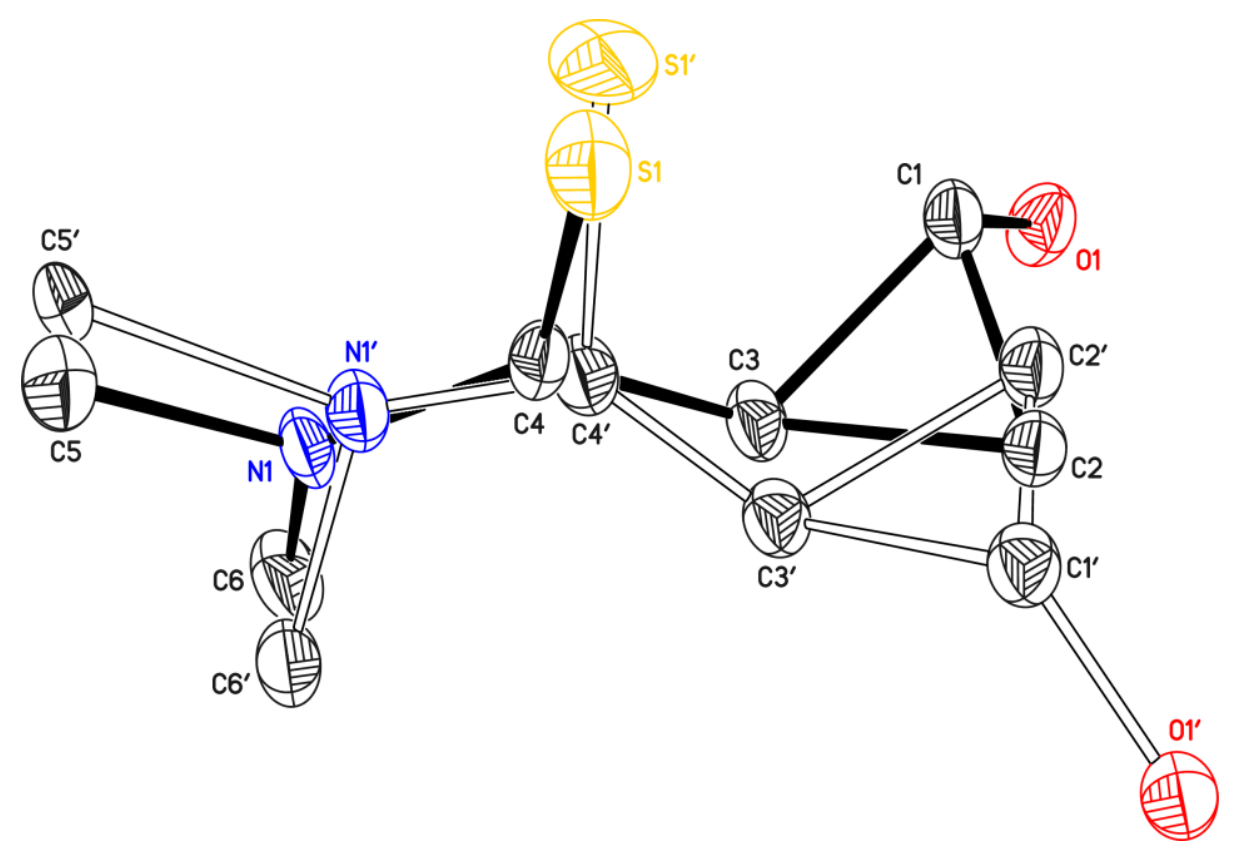

\begin{tabular}{|c|c|c|c|}
\hline Structure code & DK_B1CS1_58 & $Z$ & 4 \\
\hline Empirical formula & $\mathrm{C}_{12} \mathrm{H}_{18} \mathrm{~N}_{2} \mathrm{OS}_{2}$ & Crystal size $\left[\mathrm{mm}^{3}\right]$ & $0.20 \times 0.15 \times 0.05$ \\
\hline Formula weight [gmol-1] & 270.40 & $\rho_{\text {calc }}\left[\mathrm{Mgm}^{-3}\right]$ & 1.364 \\
\hline Temperature $[\mathrm{K}]$ & $100(2)$ & $\mu\left[\mathrm{mm}^{-1}\right]$ & 0.390 \\
\hline Wavelength $[\AA ̊]$ & 0.71073 & $F(000)$ & 576 \\
\hline Crystal system & Orthorhombic & $\Theta$ range $\left[{ }^{\circ}\right]$ & 2.925 to 25.300 \\
\hline Space group & $A b a 2$ & Reflections collected & 7020 \\
\hline Unit cell dimensions $[\AA ̊]$ & & Unique reflections & 1171 \\
\hline$a=$ & $13.926(7)$ & $R_{\text {int }} / R_{\sigma}$ & $0.0289 / 0.0209$ \\
\hline$b=$ & $12.578(5)$ & Completeness to $\theta_{\max }$ & $99.8 \%$ \\
\hline$c=$ & $7.516(4)$ & restraints/parameters & 299 / 160 \\
\hline$\alpha=$ & $90^{\circ}$ & GooF & 1.180 \\
\hline$\beta=$ & $90^{\circ}$ & $\mathrm{R} 1[\mathrm{I}>2 \sigma(\mathrm{I})]$ & 0.0276 \\
\hline$\gamma=$ & $90^{\circ}$ & wR2 (all data) & 0.0693 \\
\hline Volume $\left[\AA^{3}\right]$ & $1316.4(11)$ & diff. peak / hole $\left[\mathrm{e}^{-3}\right]$ & $0.213 /-0.161$ \\
\hline Resolution $[\AA]]$ & 0.832 & Flack $x$ & $0.07(4)$ \\
\hline
\end{tabular}

The structure turned out to be disordered over two positions with $89 \%$ (black lines) and $11 \%$ occupation. Similarity restraints for bond lengths and thermal parameters have been applied to stabilize the refinement of the minor compound. Hydrogen atoms have been omitted in the picture. 


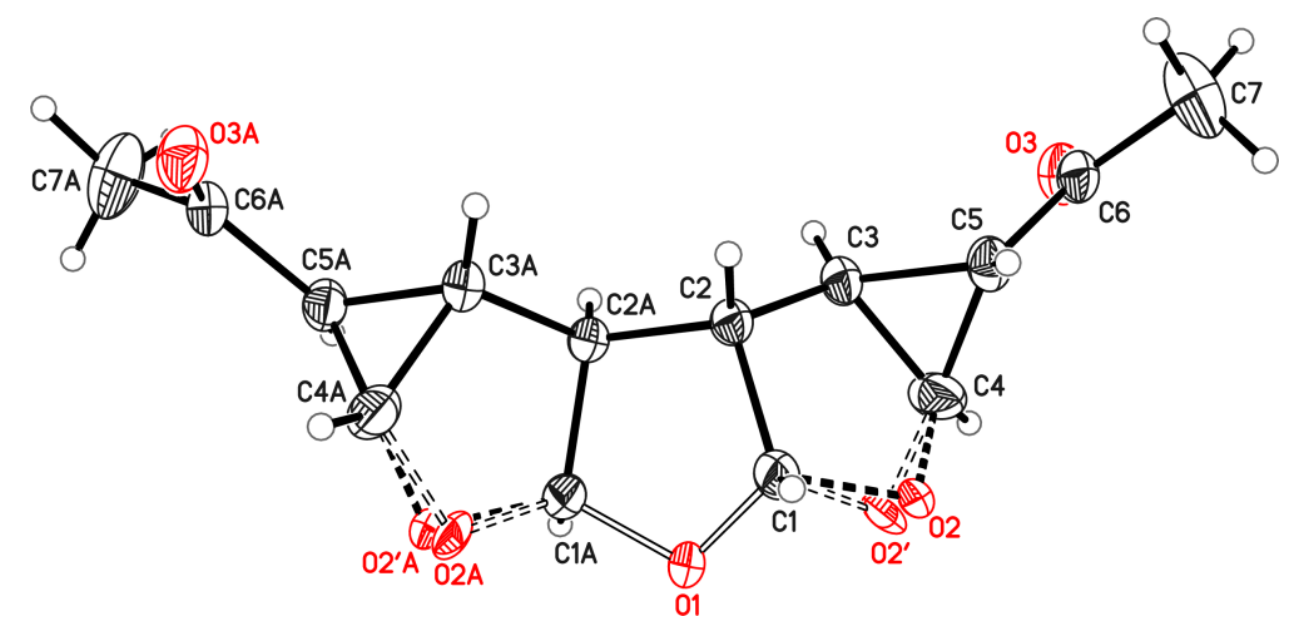

\begin{tabular}{|c|c|c|c|}
\hline Structure code & DK_JKE92 & Z & 8 \\
\hline Empirical formula & $\mathrm{C}_{14} \mathrm{H}_{16} \mathrm{O}_{5}$ & Crystal size $\left[\mathrm{mm}^{3}\right]$ & $0.24 \times 0.12 \times 0.12$ \\
\hline Formula weight [gmol-1] & 264.27 & $\rho_{\text {calc }}\left[\mathrm{Mgm}^{-3}\right]$ & 1.389 \\
\hline Temperature $[\mathrm{K}]$ & $100(2)$ & $\mu\left[\mathrm{mm}^{-1}\right]$ & 0.106 \\
\hline Wavelength $[\AA ̊]$ & 0.71073 & $F(000)$ & 1120 \\
\hline Crystal system & Orthorhombic & $\Theta$ range $\left[{ }^{\circ}\right]$ & 2.71 to 26.73 \\
\hline Space group & Fdd2 & Reflections collected & 11882 \\
\hline Unit cell dimensions $[\AA ̊]$ & & Unique reflections & 741 \\
\hline$a=$ & $18.9326(13)$ & $R_{\text {int }} / R_{\sigma}$ & $0.0260 / 0.0108$ \\
\hline$b=$ & $24.746(5)$ & Completeness to $\theta_{\max }$ & 99.7 \\
\hline$c=$ & $5.394(7)$ & restraints/parameters & $23 / 106$ \\
\hline$\alpha=$ & $90^{\circ}$ & GooF & 1.101 \\
\hline$\beta=$ & $90^{\circ}$ & $\mathrm{R} 1[\mathrm{I}>2 \sigma(\mathrm{I})]$ & 0.0355 \\
\hline$\gamma=$ & $90^{\circ}$ & wR2 (all data) & 0.0957 \\
\hline Volume $\left[\AA^{3}\right]$ & $2527(3)$ & diff. peak / hole $\left[\mathrm{e} \AA^{-3}\right]$ & $0.225 /-0.166$ \\
\hline Resolution $[\AA]]$ & 0.790 & Flack $\mathrm{x}$ & - \\
\hline
\end{tabular}

The structure could also be solved and refined as disorder of the whole molecule in Cc. A solution in Fdd 2 turned out to refine more stable. The absolute structure could not be determined due to the weak anomalous signal of oxygen with Mo radiation. 


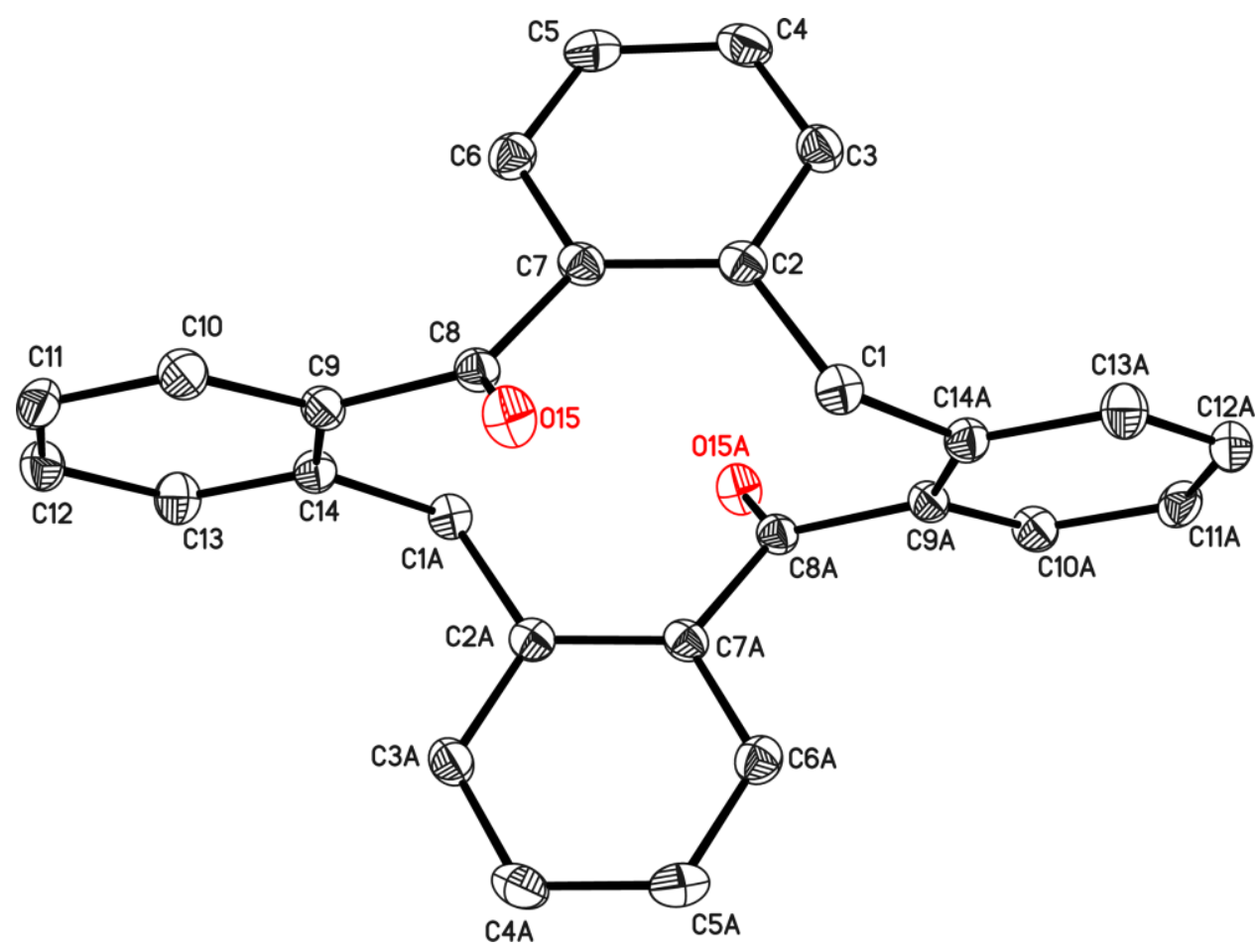

\begin{tabular}{|c|c|c|c|}
\hline Structure code & DK_ST3_11a & $\bar{Z}$ & 2 \\
\hline Empirical formula & $\mathrm{C}_{28} \mathrm{H}_{20} \mathrm{O}_{2}$ & Crystal size $\left[\mathrm{mm}^{3}\right]$ & $0.28 \times 0.16 \times 0.14$ \\
\hline Formula weight [gmol-1] & 388.44 & $\rho_{\text {calc }}\left[\mathrm{Mgm}^{-3}\right]$ & 1.363 \\
\hline Temperature $[\mathrm{K}]$ & $100(2)$ & $\mu\left[\mathrm{mm}^{-1}\right]$ & 0.084 \\
\hline Wavelength $[\AA ̊]$ & 0.71073 & $F(000)$ & 408 \\
\hline Crystal system & Monoclinic & $\Theta$ range $\left[{ }^{\circ}\right]$ & 2.51 to 28.28 \\
\hline Space group & $P 2_{1} / n$ & Reflections collected & 18264 \\
\hline Unit cell dimensions $[\AA ̊]$ & & Unique reflections & 2353 \\
\hline$a=$ & $10.7358(3)$ & $R_{\text {int }} / R_{\sigma}$ & $0.0287 / 0.0169$ \\
\hline$b=$ & $7.6505(2)$ & Completeness to $\theta_{\max }$ & $100.0 \%$ \\
\hline$c=$ & $11.5494(3)$ & restraints/parameters & $0 / 137$ \\
\hline$\alpha=$ & $90^{\circ}$ & GooF & 1.131 \\
\hline$\beta=$ & $93.7500(10)^{\circ}$ & $\mathrm{R} 1[\mathrm{I}>2 \sigma(\mathrm{I})]$ & 0.0443 \\
\hline$\gamma=$ & $90^{\circ}$ & wR2 (all data) & 0.1161 \\
\hline Volume $\left[\AA^{3}\right]$ & $946.57(4)$ & diff. peak / hole $\left[\mathrm{e} \AA^{-3}\right]$ & $0.433 /-0.262$ \\
\hline Resolution $[\AA]$ & 0.750 & & \\
\hline
\end{tabular}

The structure was a non-merohedraly twinned by rotation about $180^{\circ}$. The data was integrated with two domains and the scaling, absorption correction and merging was done with TWINABS. The refinement against HKLF 5 data resulted in a twin fraction of 0.441 . 


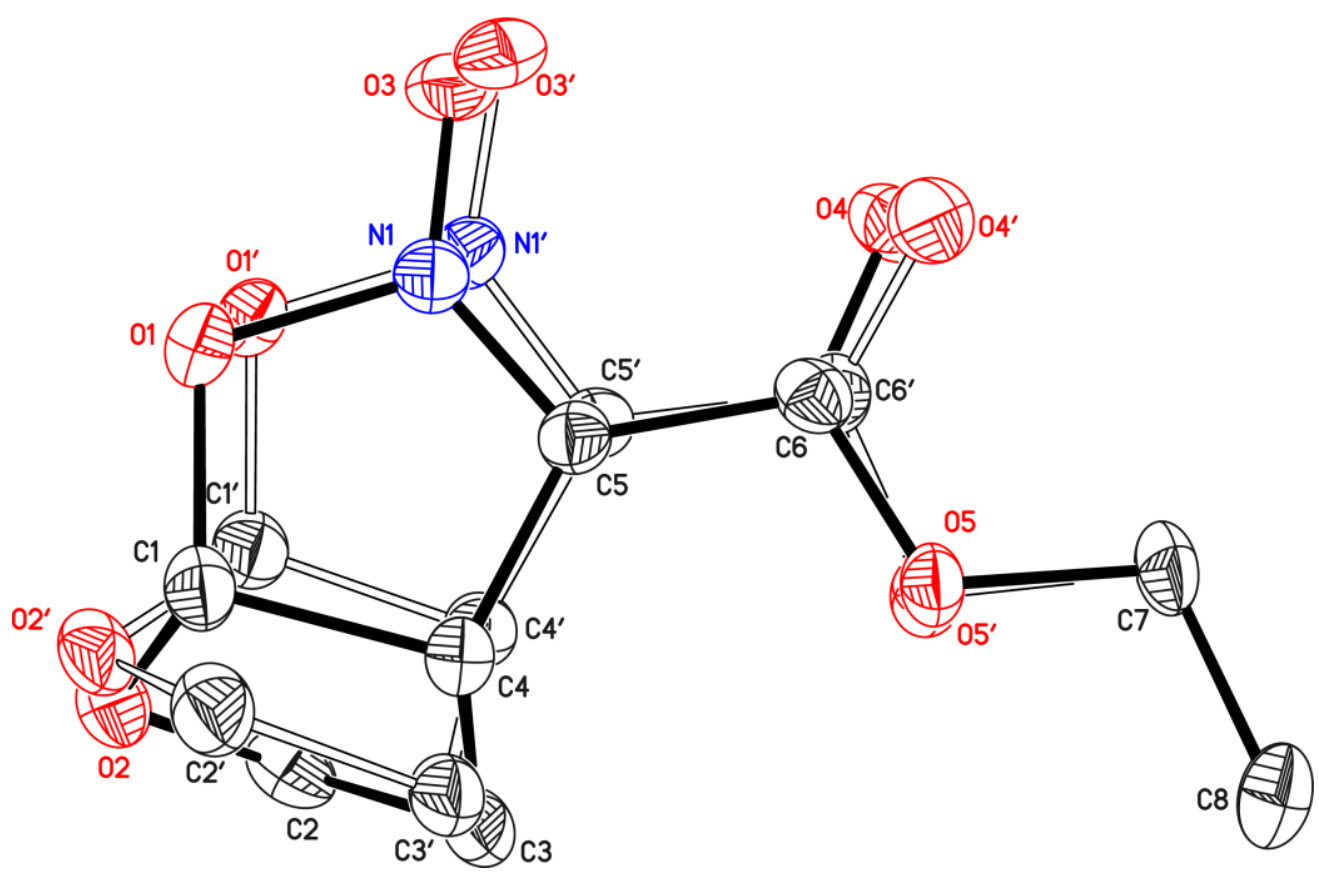

\begin{tabular}{|c|c|c|c|}
\hline Structure code & DK_JKC17 & $z$ & 4 \\
\hline Empirical formula & $\mathrm{C}_{8} \mathrm{H}_{11} \mathrm{NO}_{5}$ & Crystal size $\left[\mathrm{mm}^{3}\right]$ & $0.1 \times 0.05 \times 0.01$ \\
\hline Formula weight [gmol-1] & 201.18 & $\rho_{\text {calc }}\left[\mathrm{Mgm}^{-3}\right]$ & 1.470 \\
\hline Temperature $[\mathrm{K}]$ & $100(2)$ & $\mu\left[\mathrm{mm}^{-1}\right]$ & 0.124 \\
\hline Wavelength $[\AA ̊]$ & 0.71073 & $F(000)$ & 424 \\
\hline Crystal system & Monoclinic & $\Theta$ range $\left[{ }^{\circ}\right]$ & 2.35 to 28.38 \\
\hline Space group & $P 2 \sqrt{ } / c$ & Reflections collected & 15346 \\
\hline Unit cell dimensions $[\AA ̊]$ & & Unique reflections & 2271 \\
\hline$a$ & $7.8071(18)$ & $R_{\text {int }} / R_{\sigma}$ & $0.0423 / 0.0272$ \\
\hline$b$ & $17.361(5)$ & Completeness to $\theta_{\max }$ & $99.7 \%$ \\
\hline$c$ & $6.722(2)$ & restraints/parameters & $279 / 201$ \\
\hline$\alpha$ & $90^{\circ}$ & GooF & 1.132 \\
\hline$\beta$ & $93.918(4)^{\circ}$ & $\mathrm{R} 1[\mathrm{I}>2 \sigma(\mathrm{I})]$ & 0.0521 \\
\hline$\gamma$ & $90^{\circ}$ & wR2 (all data) & 0.1431 \\
\hline Volume $\left[\AA^{3}\right]$ & $909.0(4)$ & diff. peak / hole $\left[\mathrm{e}^{-3}\right]$ & $0.362 /-0.354$ \\
\hline Resolution $[\AA ̊]$ & 0.748 & & \\
\hline
\end{tabular}

The structure turned out to be disordered over two positions with $94.8 \%$ (black lines) and $5.2 \%$ occupation. Similarity restraints for bond lengths and thermal parameters have been applied to stabilize the refinement of the minor compound. Hydrogen atoms have been omitted in the picture. 


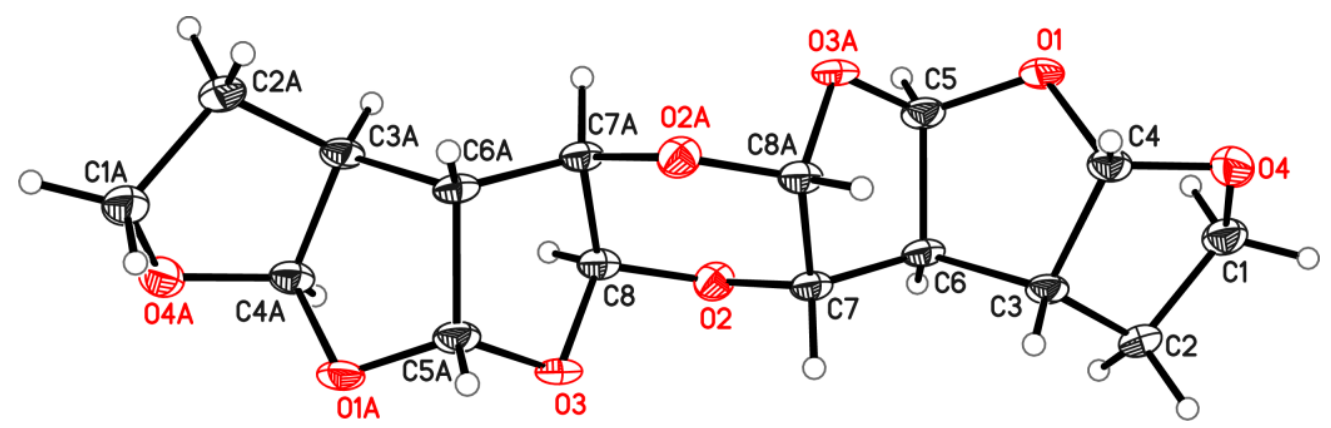

\begin{tabular}{|c|c|c|c|}
\hline Structure code & DK_ST1_01 & $z$ & 2 \\
\hline Empirical formula & $\mathrm{C}_{16} \mathrm{H}_{20} \mathrm{O}_{8}$ & Crystal size $\left[\mathrm{mm}^{3}\right]$ & $0.1 \times 0.1 \times 0.02$ \\
\hline Formula weight [gmol-1] & 340.32 & $\rho_{\text {calc }}\left[\mathrm{Mgm}^{-3}\right]$ & 1.580 \\
\hline Temperature $[\mathrm{K}]$ & $100(2)$ & $\mu\left[\mathrm{mm}^{-1}\right]$ & 0.128 \\
\hline Wavelength $[\AA]$ & 0.71073 & $F(000)$ & 360 \\
\hline Crystal system & Monoclinic & $\Theta$ range $\left[{ }^{\circ}\right]$ & 1.89 to 26.38 \\
\hline Space group & $P 2_{1} / c$ & Reflections collected & 7892 \\
\hline Unit cell dimensions $[\AA ̊]$ & & Unique reflections & 1458 \\
\hline$a$ & $11.3321(7)$ & $R_{\text {int }} / R_{\sigma}$ & $0.0446 / 0.0310$ \\
\hline$b$ & $5.7864(3)$ & Completeness to $\theta_{\max }$ & 99.7 \\
\hline$c$ & $11.4598(7)$ & restraints/parameters & $10 / 139$ \\
\hline$\alpha$ & $90^{\circ}$ & GooF & 1.085 \\
\hline$\beta$ & $107.885(4)^{\circ}$ & $\mathrm{R} 1[\mathrm{I}>2 \sigma(\mathrm{I})]$ & 0.0439 \\
\hline$\gamma$ & $90^{\circ}$ & wR2 (all data) & 0.1178 \\
\hline Volume $\left[\AA^{3}\right]$ & $715.13(7)$ & diff. peak / hole $\left[\mathrm{e} \AA^{-3}\right]$ & 0.369 and -0.264 \\
\hline Resolution $[\AA ̊]$ & 0.800 & & \\
\hline
\end{tabular}




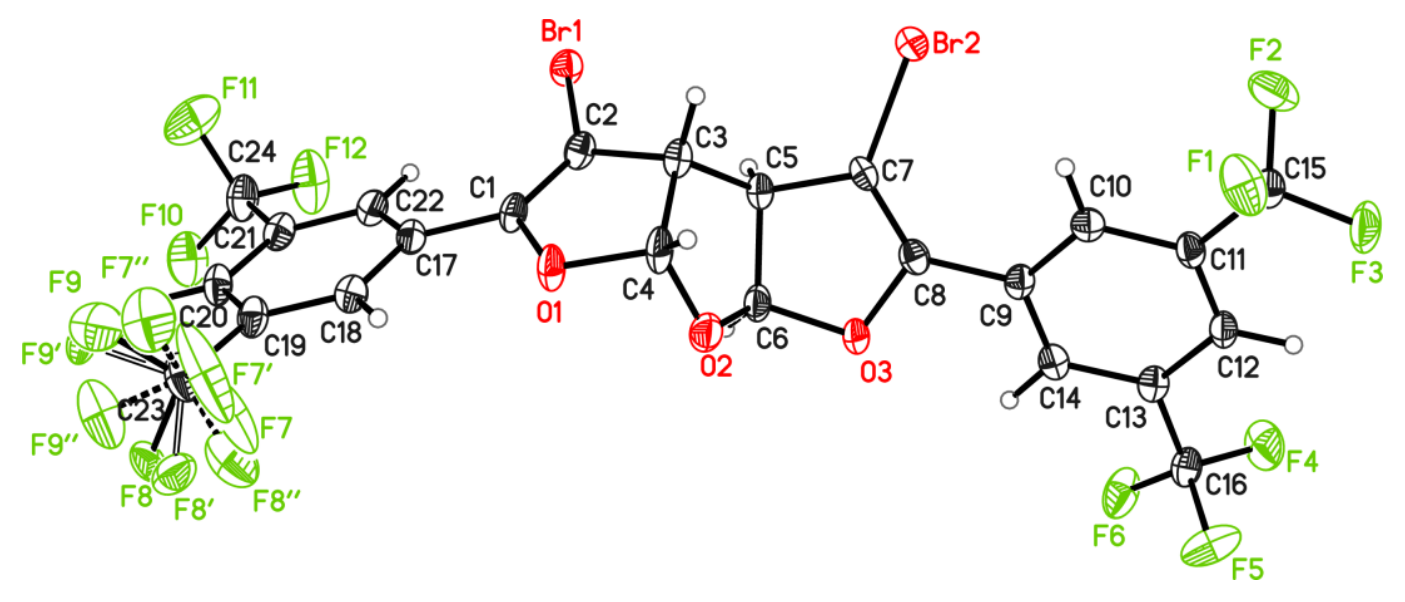

\begin{tabular}{|c|c|c|c|}
\hline Structure code & DK_ST2_52 & Z & 2 \\
\hline Empirical formula & $\mathrm{C}_{24} \mathrm{H}_{10} \mathrm{Br}_{2} \mathrm{~F}_{12} \mathrm{O}_{3}$ & Crystal size $\left[\mathrm{mm}^{3}\right]$ & $0.1 \times 0.1 \times 0.09$ \\
\hline Formula weight [gmol-1] & 734.14 & $\rho_{\text {calc }}\left[\mathrm{Mgm}^{-3}\right]$ & 2.011 \\
\hline Temperature [K] & $100(2)$ & $\mu\left[\mathrm{mm}^{-1}\right]$ & 3.464 \\
\hline Wavelength $[\AA ̊]$ & 0.71073 & $F(000)$ & 712 \\
\hline Crystal system & Triclinic & $\Theta$ range $\left[{ }^{\circ}\right]$ & 1.78 to 26.37 \\
\hline Space group & $P \overline{1}$ & Reflections collected & 55706 \\
\hline Unit cell dimensions $[\AA ̊]$ & & Unique reflections & 9323 \\
\hline$a=$ & $9.4684(2)$ & $R_{\text {int }} / R_{\sigma}$ & $0.0363 / 0.0283$ \\
\hline$b=$ & $11.1606(3)$ & Completeness to $\theta_{\max }$ & $100.0 \%$ \\
\hline$c=$ & $12.0153(3)$ & restraints/parameters & $244 / 428$ \\
\hline$\alpha=$ & $100.8790(10)^{\circ}$ & GooF & 1.090 \\
\hline$\beta=$ & $103.2650(10)^{\circ}$ & $\mathrm{R} 1[\mathrm{I}>2 \sigma(\mathrm{I})]$ & 0.0332 \\
\hline$\gamma=$ & $90.0680(10)^{\circ}$ & wR2 (all data) & 0.0921 \\
\hline Volume $\left[\AA^{3}\right]$ & $1212.29(5)$ & diff. peak / hole $\left[e \AA^{-3}\right]$ & $0.851 /-0.479$ \\
\hline Resolution [Å] & 0.800 & & \\
\hline
\end{tabular}

The crystal was non-merohedrally twinned. The structure was refined against HKLF 5 data with a batch scale factor of 0.433 . 


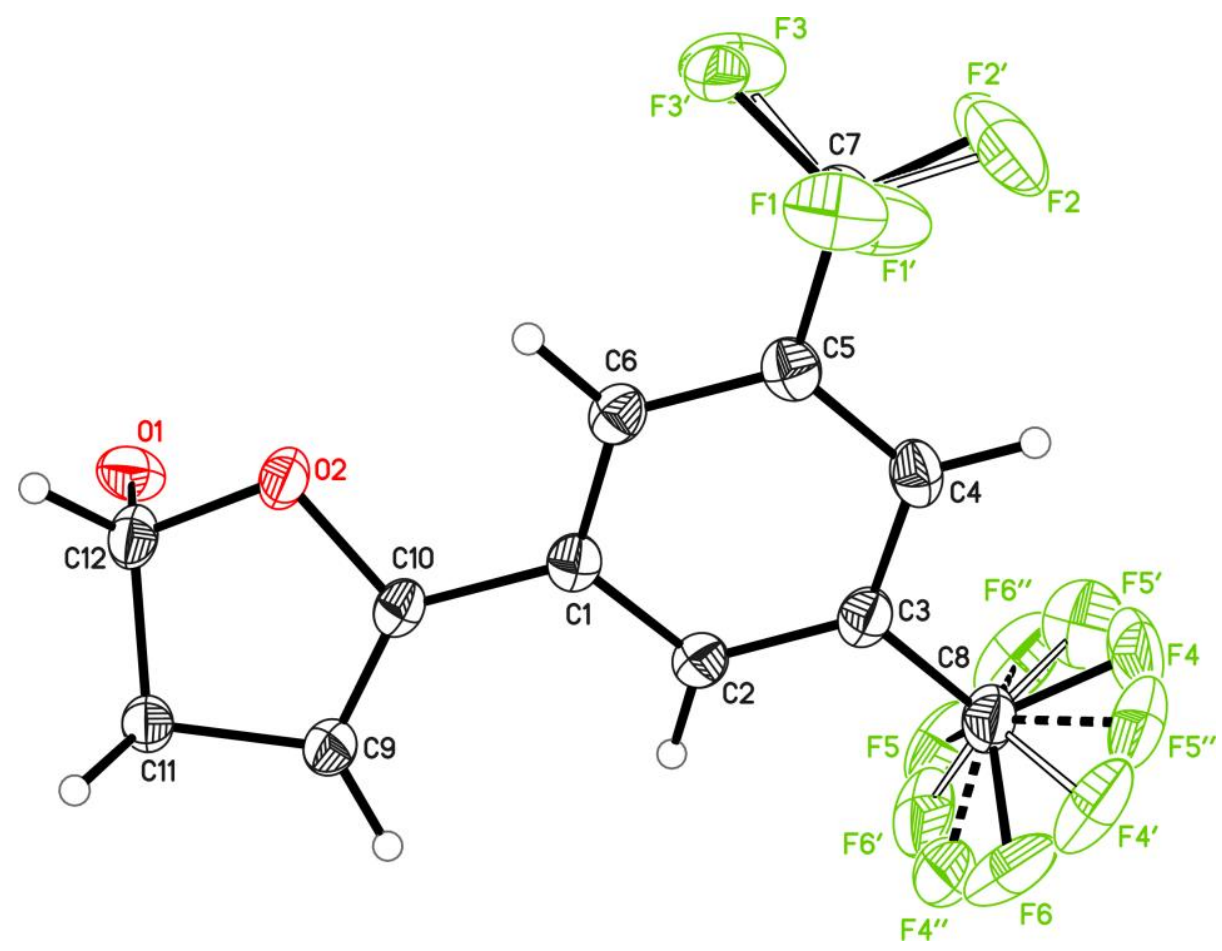

\begin{tabular}{|c|c|c|c|}
\hline Structure code & DK_ST2_27 & Z & 4 \\
\hline Empirical formula & $\mathrm{C}_{24} \mathrm{H}_{12} \mathrm{~F}_{12} \mathrm{O}_{3}$ & Crystal size $\left[\mathrm{mm}^{3}\right]$ & $0.15 \times 0.15 \times 0.1$ \\
\hline Formula weight [gmol-1] & 575.15 & $\rho_{\text {calc }}\left[\mathrm{Mgm}^{-3}\right]$ & 1.793 \\
\hline Temperature [K] & $100(2)$ & $\mu\left[\mathrm{mm}^{-1}\right]$ & 0.187 \\
\hline Wavelength $[\AA ̊]$ & 0.71073 & $F(000)$ & 1150 \\
\hline Crystal system & Monoclinic & $\Theta$ range $\left[{ }^{\circ}\right]$ & 1.24 to 27.20 \\
\hline Space group & $C 2 / c$ & Reflections collected & 24529 \\
\hline Unit cell dimensions $[\AA ̊]$ & & Unique reflections & 2345 \\
\hline$a=$ & $33.77(2)$ & $R_{\text {int }} / R_{\sigma}$ & $0.0554 / 0.0284$ \\
\hline$b=$ & $5.477(4)$ & Completeness to $\theta_{\max }$ & $99.2 \%$ \\
\hline$c=$ & $11.834(8)$ & restraints/parameters & $353 / 265$ \\
\hline$\alpha=$ & $90^{\circ}$ & GooF & 1.075 \\
\hline$\beta=$ & $103.211(12)^{\circ}$ & $\mathrm{R} 1[\mathrm{I}>2 \sigma(\mathrm{I})]$ & 0.0424 \\
\hline$\gamma=$ & $90^{\circ}$ & wR2 (all data) & 0.0987 \\
\hline Volume $\left[\AA^{3}\right]$ & $2131(2)$ & diff. peak / hole $\left[\mathrm{e}^{-3}\right]$ & $0.273 /-0.282$ \\
\hline Resolution [Å] & 0.777 & & \\
\hline
\end{tabular}



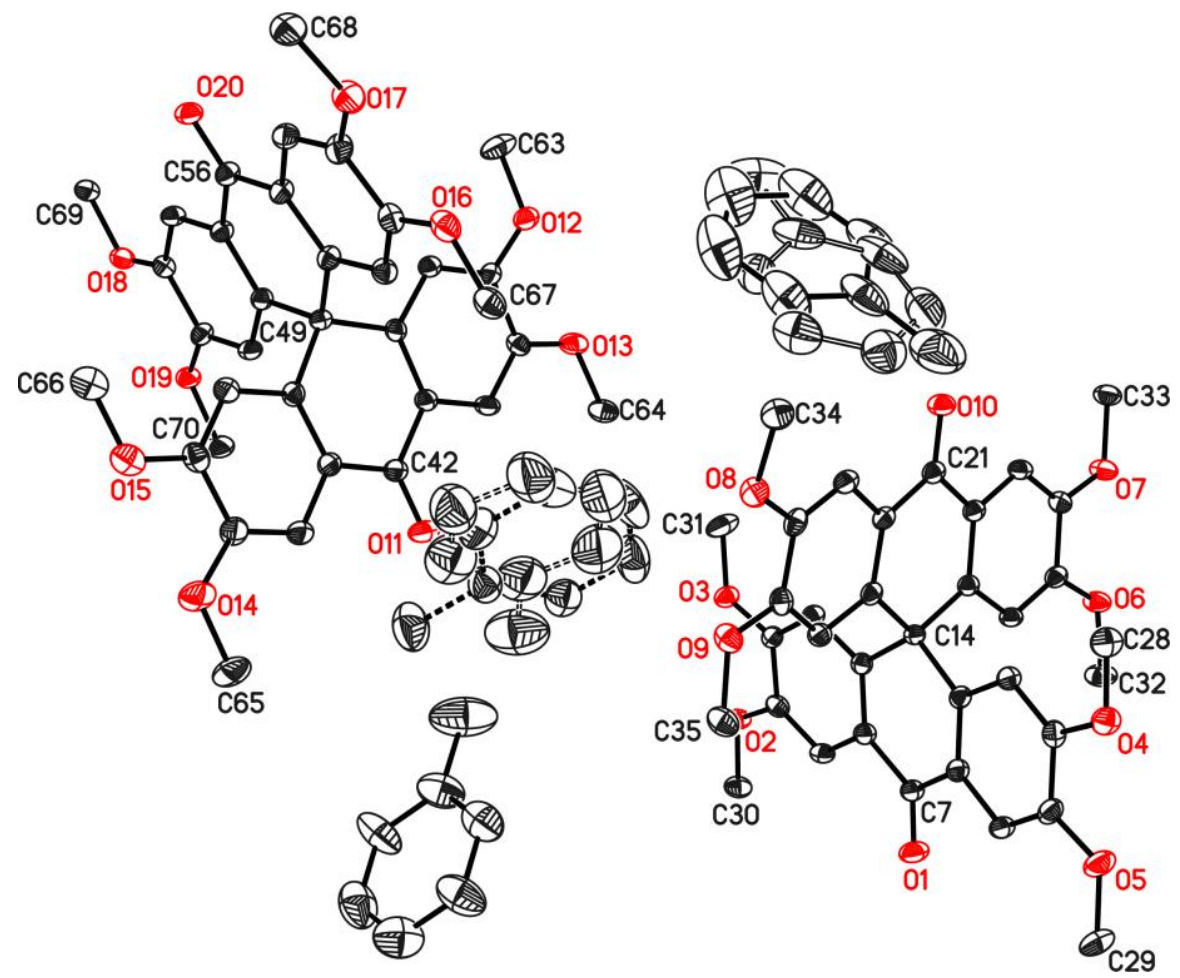

\begin{tabular}{|c|c|c|c|}
\hline Structure code & DK_ST2_X02 & Z & 4 \\
\hline Empirical formula & $\mathrm{C}_{91} \mathrm{H}_{88} \mathrm{O}_{20}$ & Crystal size $\left[\mathrm{mm}^{3}\right]$ & $0.22 \times 0.13 \times 0.07$ \\
\hline Formula weight [gmol-1] & 1501.61 & $\rho_{\text {calc }}\left[\mathrm{Mgm}^{-3}\right]$ & 1.292 \\
\hline Temperature $[\mathrm{K}]$ & $100(2)$ & $\mu\left[\mathrm{mm}^{-1}\right]$ & 0.091 \\
\hline Wavelength $[\AA]$ & 0.71073 & $F(000)$ & 3176 \\
\hline Crystal system & Monoclinic & $\Theta$ range $\left[{ }^{\circ}\right]$ & 1.26 to 26.73 \\
\hline Space group & $P 2_{1} / c$ & Reflections collected & 342741 \\
\hline Unit cell dimensions $[\AA ̊]$ & & Unique reflections & 16388 \\
\hline$a=$ & $16.6171(14)$ & $\mathrm{R}_{\text {int }} / \mathrm{R}_{\sigma}$ & 0.047570 .0168 \\
\hline$b=$ & $16.4743(12)$ & Completeness to $\theta_{\max }$ & $100.0 \%$ \\
\hline$c=$ & $28.964(3)$ & restraints/parameters & $291 / 1150$ \\
\hline$\alpha=$ & $90^{\circ}$ & GooF & 1.019 \\
\hline$\beta=$ & $103.218(4)^{\circ}$ & $\mathrm{R} 1[\mathrm{I}>2 \sigma(\mathrm{I})]$ & 0.0420 \\
\hline$\gamma=$ & $90^{\circ}$ & wR2 (all data) & 0.1204 \\
\hline Volume $\left[\AA^{3}\right]$ & $7719.0(12)$ & diff. peak / hole $\left[\mathrm{e} \AA^{-3}\right]$ & $0.313 /-0.220$ \\
\hline Resolution $[\AA]$ & 0.790 & & \\
\hline
\end{tabular}




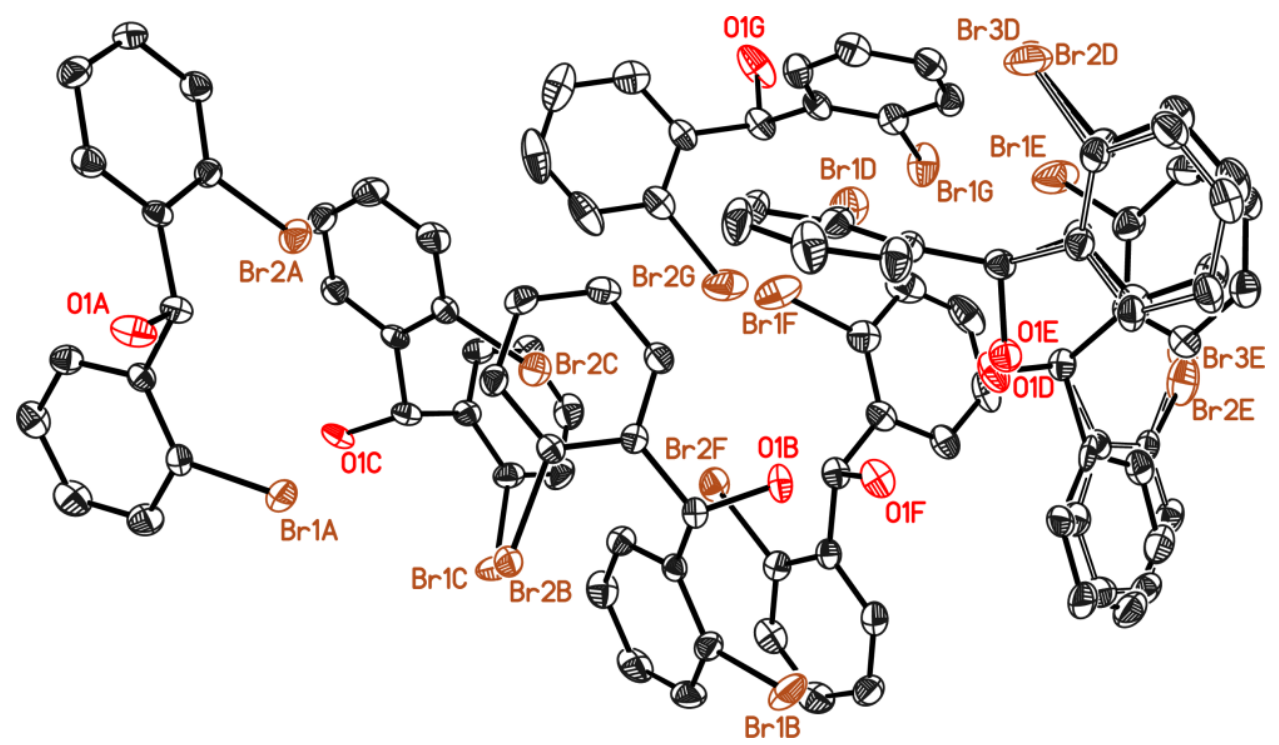

\begin{tabular}{|c|c|c|c|}
\hline Structure code & DK_ST2_54b & $Z / Z^{\prime}$ & $14 / 7$ \\
\hline Empirical formula & $\mathrm{C}_{13} \mathrm{H}_{10} \mathrm{Br}_{2} \mathrm{O}$ & Crystal size $\left[\mathrm{mm}^{3}\right]$ & $0.18 \times 0.17 \times 0.15$ \\
\hline Formula weight [gmol-1] & 342.03 & $\rho_{\text {calc }}\left[\mathrm{Mgm}^{-3}\right]$ & 1.825 \\
\hline Temperature $[\mathrm{K}]$ & $100(2)$ & $\mu\left[\mathrm{mm}^{-1}\right]$ & 6.488 \\
\hline Wavelength $[\AA ̊]$ & 0.71073 & $F(000)$ & 2324 \\
\hline Crystal system & Triclinic & $\Theta$ range $\left[{ }^{\circ}\right]$ & 1.70 to 26.72 \\
\hline Space group & $P \overline{1}$ & Reflections collected & 108627 \\
\hline Unit cell dimensions $[\AA ̊]$ & & Unique reflections & 18430 \\
\hline$a=$ & $10.8849(10)$ & $R_{\text {int }} / R_{\sigma}$ & $0.0358 / 0.0252$ \\
\hline$b=$ & $13.1490(12)$ & Completeness to $\theta_{\max }$ & $99.8 \%$ \\
\hline$c=$ & $33.667(3)$ & restraints/parameters & $420 / 1138$ \\
\hline$\alpha=$ & $100.138(2)^{\circ}$ & GooF & 1.012 \\
\hline$\beta=$ & $91.685(2)^{\circ}$ & $\mathrm{R} 1[\mathrm{I}>2 \sigma(\mathrm{I})]$ & 0.0271 \\
\hline$\gamma=$ & $112.539(2)^{\circ}$ & wR2 (all data) & 0.0607 \\
\hline Volume $\left[\AA^{3}\right]$ & $4356.1(7)$ & diff. peak / hole $\left[e \AA^{-3}\right]$ & $1.192 /-0.996$ \\
\hline Resolution $[\AA ̊]$ & 0.790 & & \\
\hline
\end{tabular}

The structure of DK_ST2_54b features the seldom occurrence of $Z^{\prime}=7$. The molecule is flexible enough and the $\mathrm{Br}-\mathrm{O}$ contacts are strong so that the structure could crystallize with seven molecules in the asymmetric unit. 


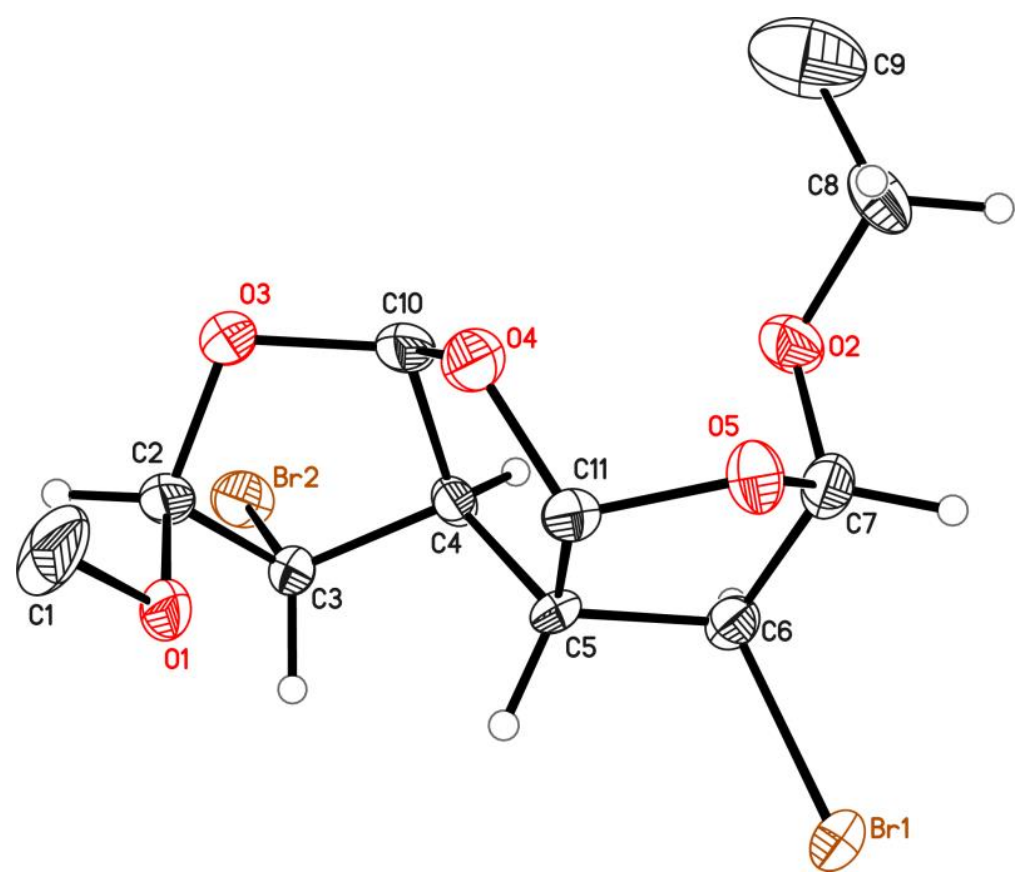

\begin{tabular}{|c|c|c|c|}
\hline Structure code & DK_ST1_48 & Z & 4 \\
\hline Empirical formula & $\mathrm{C}_{11} \mathrm{H}_{16} \mathrm{Br}_{2} \mathrm{O}_{5}$ & Crystal size $\left[\mathrm{mm}^{3}\right]$ & $0.15 \times 0.1 \times 0.1$ \\
\hline Formula weight [gmol-1] & 388.06 & $\rho_{\text {calc }}\left[\mathrm{Mgm}^{-3}\right]$ & 1.859 \\
\hline Temperature $[\mathrm{K}]$ & $100(2)$ & $\mu\left[\mathrm{mm}^{-1}\right]$ & 5.856 \\
\hline Wavelength $[\AA]$ & 0.71073 & $F(000)$ & 768 \\
\hline Crystal system & Orthorhombic & $\Theta$ range $\left[{ }^{\circ}\right]$ & 2.50 to 26.57 \\
\hline Space group & $P 2_{1} 2_{1} 2_{1}$ & Reflections collected & 25940 \\
\hline Unit cell dimensions $[\AA ̊]$ & & Unique reflections & 2887 \\
\hline$a$ & $10.2076(5)$ & $\mathrm{R}_{\text {int }} / \mathrm{R}_{\sigma}$ & $0.0870 / 0.0468$ \\
\hline$b$ & $10.5002(4)$ & Completeness to $\theta_{\max }$ & $99.6 \%$ \\
\hline$c$ & $12.9366(5)$ & restraints/parameters & $0 / 165$ \\
\hline$\alpha$ & $90^{\circ}$ & GooF & 1.068 \\
\hline$\beta$ & $90^{\circ}$ & $\mathrm{R} 1[\mathrm{I}>2 \sigma(\mathrm{I})]$ & 0.0533 \\
\hline$\gamma$ & $90^{\circ}$ & wR2 (all data) & 0.1325 \\
\hline Volume $\left[\AA^{3}\right]$ & $1386.57(10)$ & diff. peak / hole $\left[\mathrm{e} \AA^{-3}\right]$ & $1.189 /-1.010$ \\
\hline Resolution $[\AA]$ & 0.794 & Flack $\mathrm{x}$ & $0.02(3)$ \\
\hline
\end{tabular}

Methyl hydrogen atoms of $\mathrm{C} 1$ and $\mathrm{C} 9$ have been omitted. 


\subsection{Structures Measured for Patrick Zark (Prof. T. Müller)}

\subsubsection{Unpublished Structures}

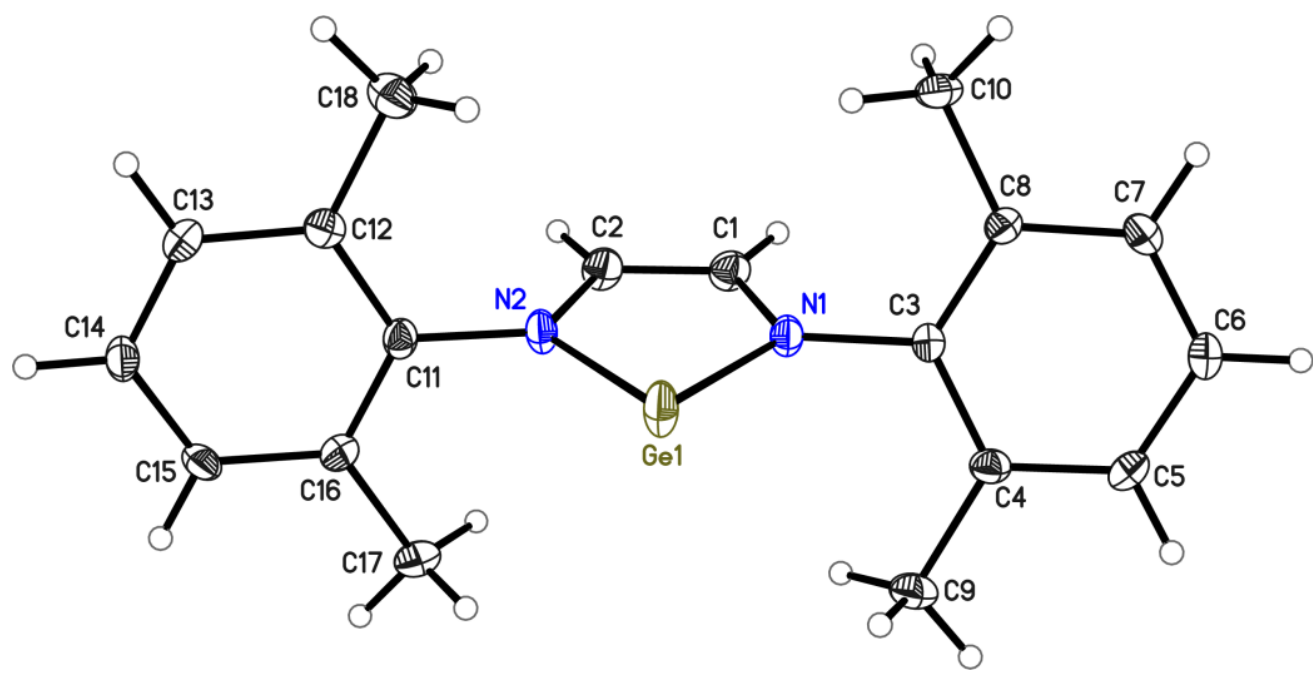

\begin{tabular}{|c|c|c|c|}
\hline Structure code & DK_Zark_Germylen1 & $Z$ & 2 \\
\hline Empirical formula & $\mathrm{C}_{18} \mathrm{H}_{20} \mathrm{GeN}_{2}$ & Crystal size $\left[\mathrm{mm}^{3}\right]$ & $0.25 \times 0.2 \times 0.18$ \\
\hline Formula weight [gmol-1] & 336.95 & $\rho_{\text {calc }}\left[\mathrm{Mgm}^{-3}\right]$ & 1.383 \\
\hline Temperature $[\mathrm{K}]$ & $100(2)$ & $\mu\left[\mathrm{mm}^{-1}\right]$ & 1.888 \\
\hline Wavelength $[\AA ̊]$ & 0.71073 & $F(000)$ & 348 \\
\hline Crystal system & Triclinic & $\Theta$ range $\left[{ }^{\circ}\right]$ & 1.99 to 30.30 \\
\hline Space group & $P \overline{1}$ & Reflections collected & 51897 \\
\hline Unit cell dimensions $[\AA ̊]$ & & Unique reflections & 4816 \\
\hline$a=$ & $8.1914(7)$ & $R_{\text {int }} / R_{\sigma}$ & $0.1082 / 0.0666$ \\
\hline$b=$ & $10.4869(8)$ & Completeness to $\theta_{\max }$ & $99.4 \%$ \\
\hline$c=$ & $11.0129(8)$ & restraints/parameters & $0 / 194$ \\
\hline$\alpha=$ & $69.759(4)^{\circ}$ & GooF & 1.055 \\
\hline$\beta=$ & $74.842(4)^{\circ}$ & $\mathrm{R} 1[\mathrm{I}>2 \sigma(\mathrm{I})]$ & 0.0402 \\
\hline$\gamma=$ & $67.204(4)^{\circ}$ & wR2 (all data) & 0.0979 \\
\hline Volume $\left[\AA^{3}\right]$ & $809.35(11)$ & diff. peak / hole $\left[\mathrm{e}^{-3}\right]$ & $0.749 /-0.675$ \\
\hline Resolution [Å] & 0.704 & & \\
\hline
\end{tabular}

The crystal was non-merohedrally twinned. The structure was refined against HKLF 4 data. Only the reflections of the stronger domain were used for refinement. 


\subsection{Structures Measured for Annika Stute (Prof. G. Erker)}

\subsubsection{Unpublished Structures}

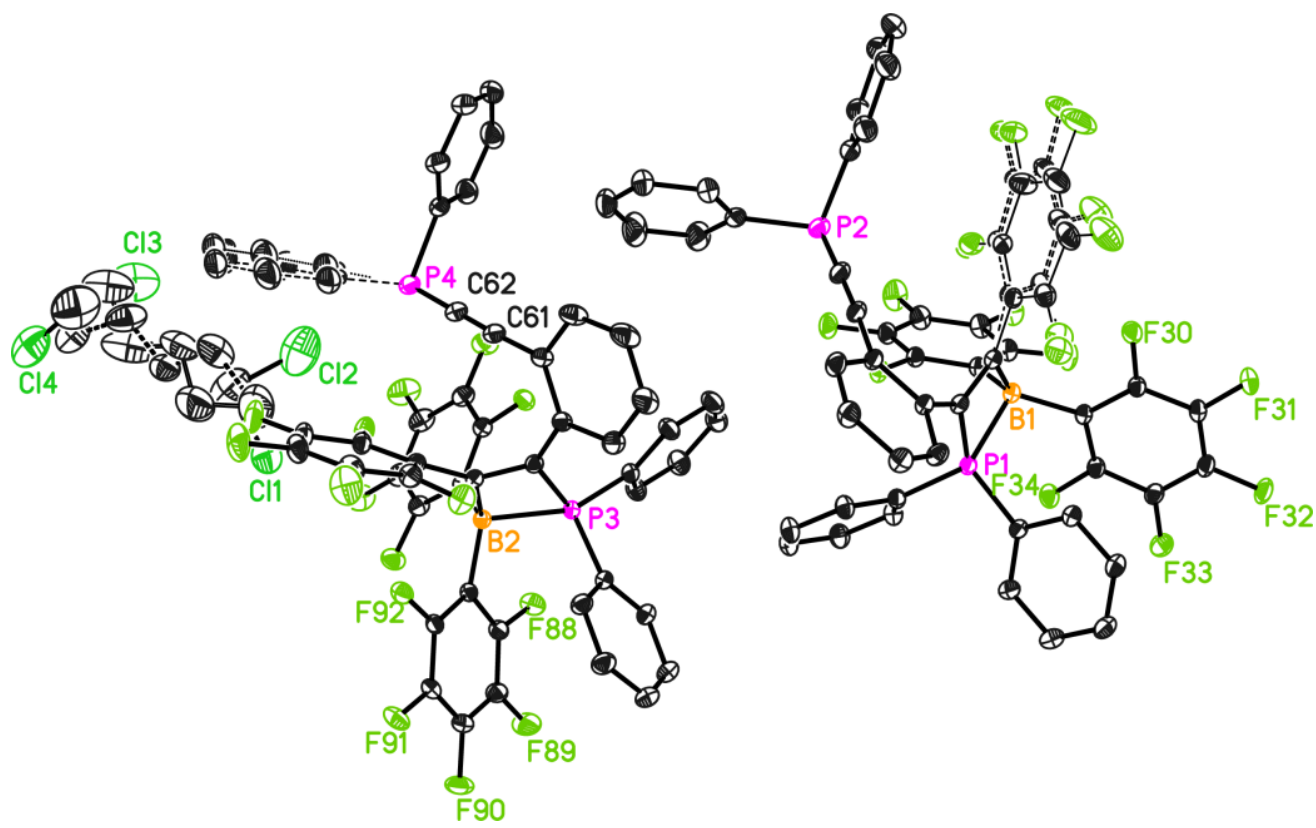

\begin{tabular}{|c|c|c|c|}
\hline Structure code & DK_REL-105 & Z & 2 \\
\hline Empirical formula & $\mathrm{C}_{109.13} \mathrm{H}_{60.25} \mathrm{~B}_{2} \mathrm{Cl}_{0.25} \mathrm{~F}_{30} \mathrm{P}_{4}$ & Crystal size $\left[\mathrm{mm}^{3}\right]$ & $0.20 \times 0.15 \times 0.12$ \\
\hline Formula weight [gmol-1] & 2096.08 & $\rho_{\text {calc }}\left[\mathrm{Mgm}^{-3}\right]$ & 1.488 \\
\hline Temperature $[\mathrm{K}]$ & $100(2)$ & $\mu\left[\mathrm{mm}^{-1}\right]$ & 0.199 \\
\hline Wavelength $[\AA ̊]$ & 0.71073 & $F(000)$ & 2119 \\
\hline Crystal system & Triclinic & $\Theta$ range $\left[{ }^{\circ}\right]$ & 1.052 to 37.051 \\
\hline Space group & $P \overline{1}$ & Reflections collected & 401291 \\
\hline Unit cell dimensions $[\AA ̊]$ & & Unique reflections & 47709 \\
\hline$a=$ & $15.073(6)$ & $R_{\text {int }} / R_{\sigma}$ & $0.0473 / 0.0213$ \\
\hline$b=$ & $16.045(7)$ & Completeness to $\theta_{\max }$ & $100.0 \%$ \\
\hline$c=$ & 20.933(9) & restraints/parameters & 1411 / 1484 \\
\hline$\alpha=$ & $98.01(2)^{\circ}$ & GooF & 1.070 \\
\hline$\beta=$ & $110.508(18)^{\circ}$ & $\mathrm{R} 1[\mathrm{I}>2 \sigma(\mathrm{I})]$ & 0.0399 \\
\hline$\gamma=$ & $91.58(2)^{\circ}$ & wR2 (all data) & 0.1193 \\
\hline Volume $\left[\AA^{3}\right]$ & $4680(3)$ & diff. peak / hole $\left[\mathrm{e} \AA^{-3}\right]$ & $0.674 /-0.472$ \\
\hline Resolution $[\AA ̊]$ & 0.590 & & \\
\hline
\end{tabular}

All hydrogen atoms have been omitted. The hexane molecule is disordered over two positions and two dichloromethane molecules with $6 \%$ occupancy were found in the difference density. One pentafluorobenzene ring was disordered over two positions and one benzene ring was also disordered over two positions. 


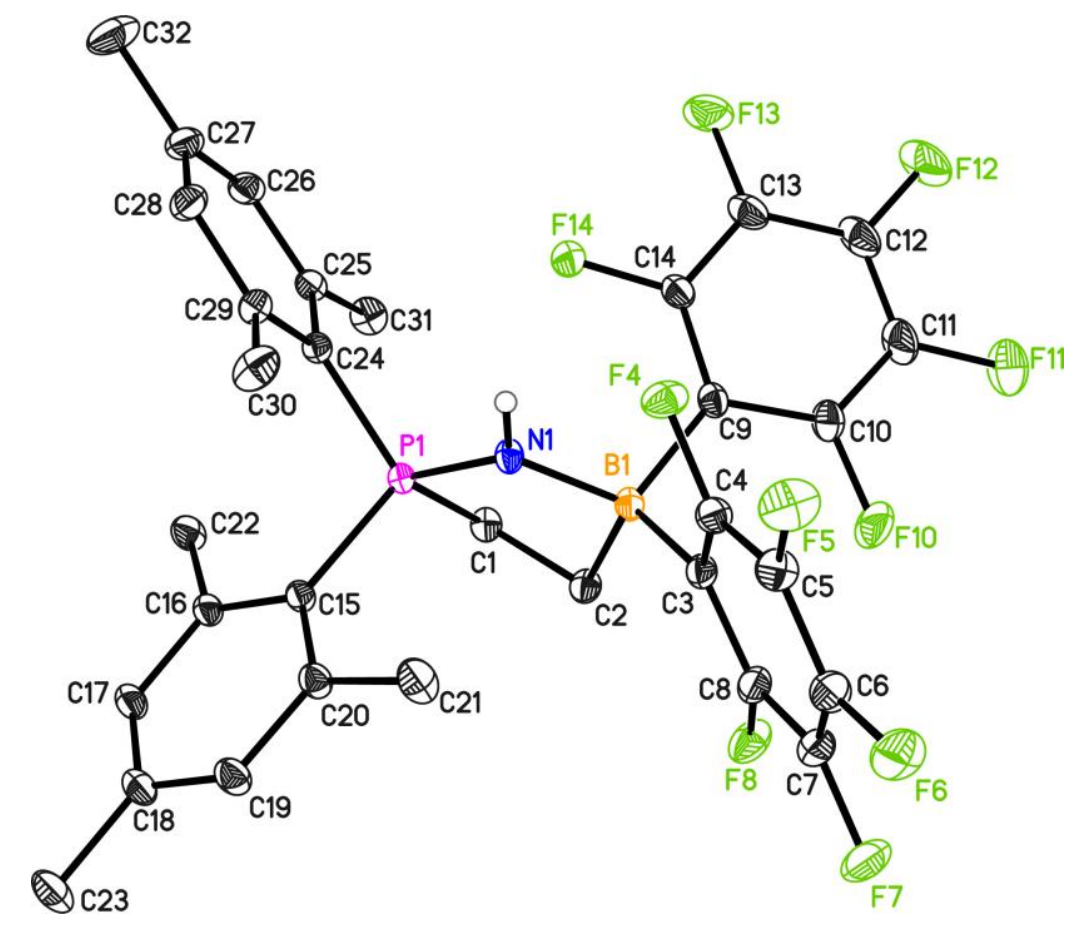

\begin{tabular}{|c|c|c|c|}
\hline Structure code & DK_Ann-255 & Z & 4 \\
\hline Empirical formula & $\mathrm{C}_{32} \mathrm{H}_{27} \mathrm{BF}_{10} \mathrm{NP}$ & Crystal size $\left[\mathrm{mm}^{3}\right]$ & $0.26 \times 0.24 \times 0.22$ \\
\hline Formula weight [gmol-1] & 657.32 & $\rho_{\text {calc }}\left[\mathrm{Mgm}^{-3}\right]$ & 1.493 \\
\hline Temperature $[\mathrm{K}]$ & $101(2)$ & $\mu\left[\mathrm{mm}^{-1}\right]$ & 0.183 \\
\hline Wavelength $[\AA ̊]$ & 0.71073 & $F(000)$ & 1344 \\
\hline Crystal system & Monoclinic & $\Theta$ range $\left[{ }^{\circ}\right]$ & 1.798 to 50.621 \\
\hline Space group & $P 2_{1} / n$ & Reflections collected & 237056 \\
\hline Unit cell dimensions $[\AA ̊]$ & & Unique reflections & 31473 \\
\hline$a=$ & $11.0432(3)$ & $R_{\text {int }} / R_{\sigma}$ & $0.0400 / 0.0180$ \\
\hline$b=$ & $14.3548(4)$ & Completeness to $\theta_{\max }$ & $99.9 \%$ \\
\hline$c=$ & $18.7544(5)$ & restraints/parameters & $1 / 419$ \\
\hline$\alpha=$ & $90^{\circ}$ & GooF & 1.051 \\
\hline$\beta=$ & $100.3690(10)^{\circ}$ & $\mathrm{R} 1[\mathrm{I}>2 \sigma(\mathrm{I})]$ & 0.0362 \\
\hline$\gamma=$ & $90^{\circ}$ & wR2 (all data) & 0.1193 \\
\hline Volume $\left[\AA^{3}\right]$ & $2924.45(14)$ & diff. peak / hole $\left[\mathrm{e} \AA^{-3}\right]$ & $0.685 /-0.334$ \\
\hline Resolution $[\AA ̊]$ & 0.460 & & \\
\hline
\end{tabular}

All hydrogens have been omitted. The methyl groups of C23, C30 and C32 turned out to be rotationally disordered over two positions. 


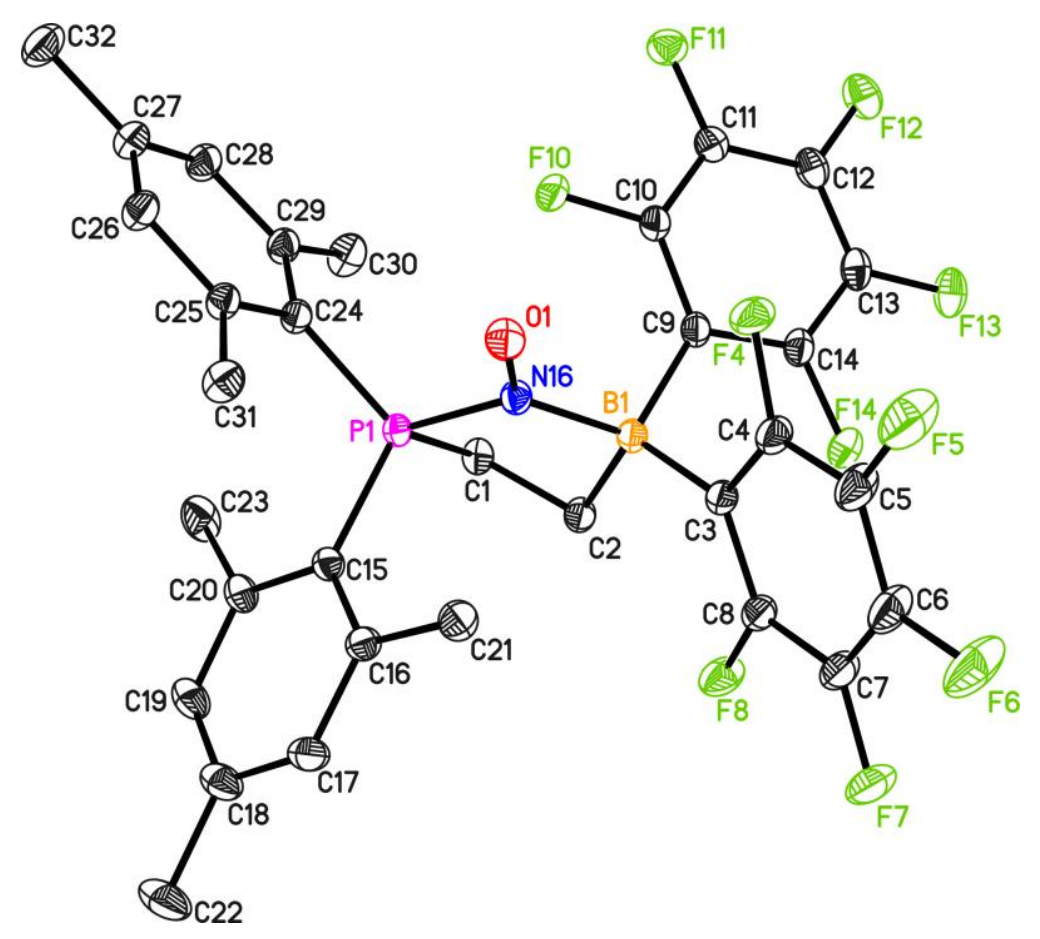

\begin{tabular}{|c|c|c|c|}
\hline Structure code & DK_Ann-205 & $\mathrm{z}$ & 8 \\
\hline Empirical formula & $\mathrm{C}_{32} \mathrm{H}_{26} \mathrm{BF}_{10} \mathrm{NOP}$ & Crystal size $\left[\mathrm{mm}^{3}\right]$ & $0.29 \times 0.27 \times 0.26$ \\
\hline Formula weight [gmol-1] & 672.32 & $\rho_{\text {calc }}\left[\mathrm{Mgm}^{-3}\right]$ & 1.513 \\
\hline Temperature $[\mathrm{K}]$ & $100(2)$ & $\mu\left[\mathrm{mm}^{-1}\right]$ & 0.185 \\
\hline Wavelength [Å] & 0.71073 & $F(000)$ & 2744 \\
\hline Crystal system & Monoclinic & $\Theta$ range $\left[{ }^{\circ}\right]$ & 1.726 to 40.261 \\
\hline Space group & $C 2 / c$ & Reflections collected & 289122 \\
\hline Unit cell dimensions $[\AA ̊]$ & & Unique reflections & 18578 \\
\hline$a=$ & $22.059(4)$ & $R_{\text {int }} / R_{\sigma}$ & $0.0301 / 0.0099$ \\
\hline$b=$ & 14.650(3) & Completeness to $\theta_{\max }$ & $100.0 \%$ \\
\hline$c=$ & $20.229(4)$ & restraints/parameters & $354 / 423$ \\
\hline$\alpha=$ & $90^{\circ}$ & GooF & 1.046 \\
\hline$\beta=$ & $115.470(10)^{\circ}$ & $\mathrm{R} 1[\mathrm{I}>2 \sigma(\mathrm{I})]$ & 0.0371 \\
\hline$\gamma=$ & $90^{\circ}$ & wR2 (all data) & 0.1193 \\
\hline Volume $\left[\AA^{3}\right]$ & $5902(2)$ & diff. peak / hole $\left[e \AA^{-3}\right]$ & $0.785 /-0.714$ \\
\hline Resolution [Å] & 0.550 & & \\
\hline
\end{tabular}




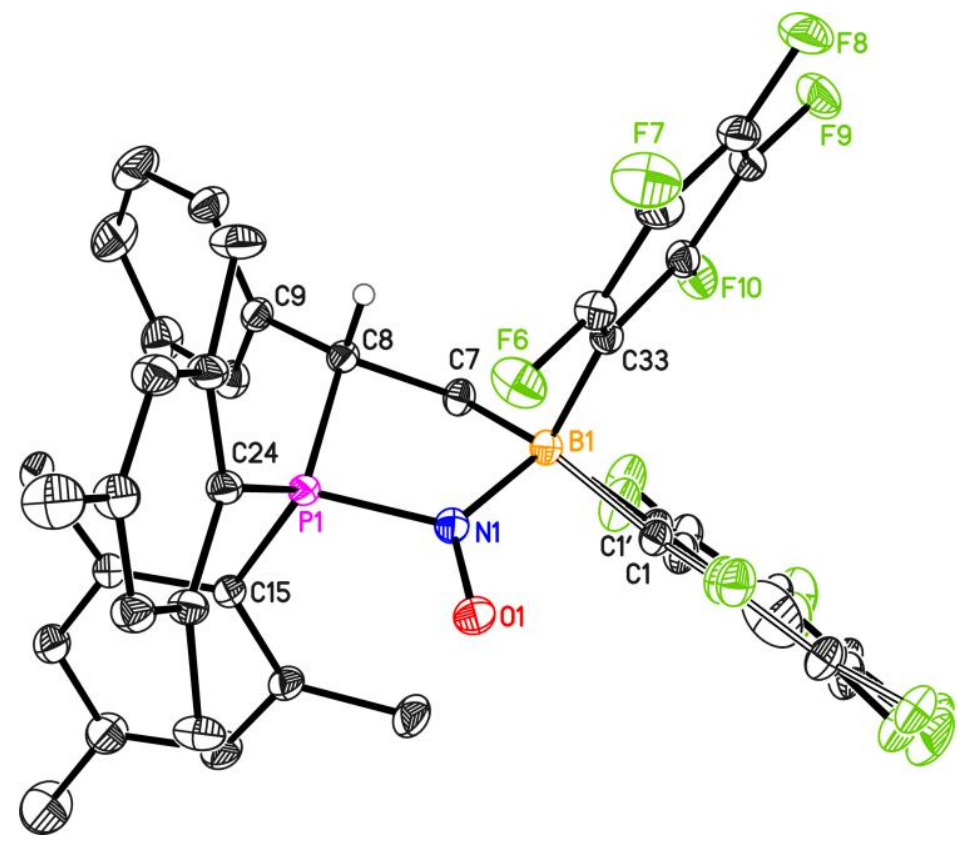

\begin{tabular}{|c|c|c|c|}
\hline Structure code & DK_MSA060 & z & 4 \\
\hline Empirical formula & $\mathrm{C}_{38} \mathrm{H}_{30} \mathrm{BF}_{10} \mathrm{NOP}$ & Crystal size $\left[\mathrm{mm}^{3}\right]$ & $0.38 \times 0.25 \times 0.14$ \\
\hline Formula weight [gmol-1] & 748.41 & $\rho_{\text {calc }}\left[\mathrm{Mgm}^{-3}\right]$ & 1.466 \\
\hline Temperature $[\mathrm{K}]$ & $100(2)$ & $\mu\left[\mathrm{mm}^{-1}\right]$ & 0.170 \\
\hline Wavelength $[\AA ̊]$ & 0.71073 & $F(000)$ & 1532 \\
\hline Crystal system & Monoclinic & $\Theta$ range $\left[{ }^{\circ}\right]$ & 1.684 to 36.467 \\
\hline Space group & $P 2_{1} / c$ & Reflections collected & 130216 \\
\hline Unit cell dimensions $[\AA ̊]$ & & Unique reflections & 16547 \\
\hline$a=$ & $11.5494(5)$ & $\mathrm{R}_{\text {int }} / \mathrm{R}_{\sigma}$ & $0.0409 / 0.0196$ \\
\hline$b=$ & $16.3699(7)$ & Completeness to $\theta_{\max }$ & $100.0 \%$ \\
\hline$c=$ & $18.6663(8)$ & restraints/parameters & $368 / 512$ \\
\hline$\alpha=$ & $90^{\circ}$ & GooF & 1.052 \\
\hline$\beta=$ & $106.073(2)^{\circ}$ & $\mathrm{R} 1[\mathrm{I}>2 \sigma(\mathrm{I})]$ & 0.0455 \\
\hline$\gamma=$ & $90^{\circ}$ & wR2 (all data) & 0.1331 \\
\hline Volume $\left[\AA^{3}\right]$ & $3391.1(3)$ & diff. peak / hole $\left[\mathrm{e} \AA^{-3}\right]$ & $1.314 /-0.313$ \\
\hline Resolution $[\AA ̊]$ & 0.598 & & \\
\hline
\end{tabular}

One of the $\mathrm{C}_{6} \mathrm{~F}_{5}$-rings turned out to be disordered. The thermal ellipsoids of both parts of the disorder were constrained to each other. The highest peak in the residual density near P1 (0.73 $)$ remained unexplainable. It might be a disorder with another isomer of the P-B-frustrated Lewis pair. 


\section{Appendix}

\subsection{Cross Hair Alignment for Bruker 3-circle Goniometers}

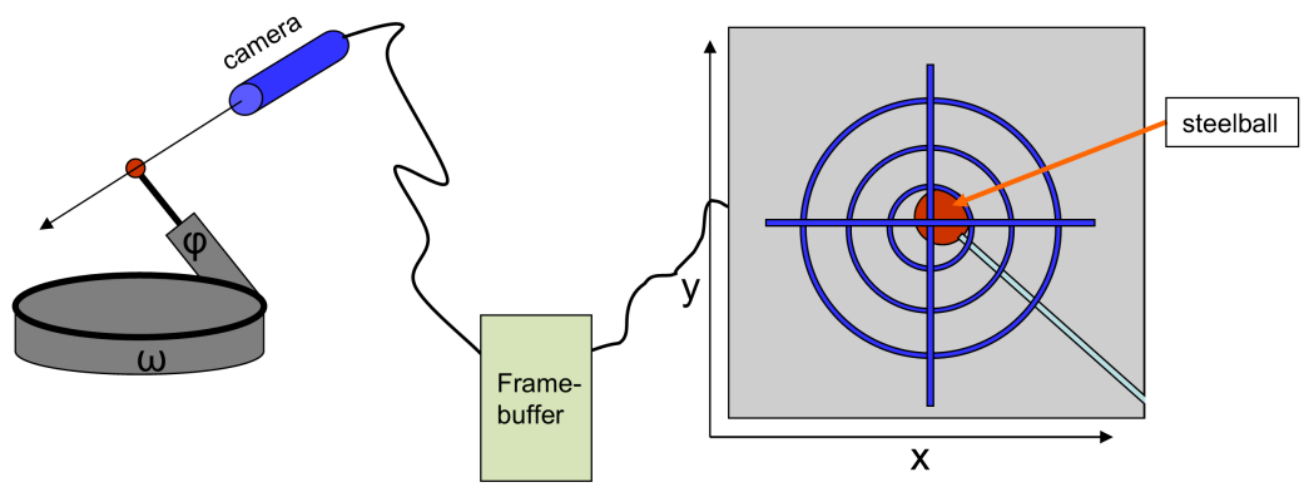

1. Center at

position 'right': - bring the steelball to the center,

rotate $\varphi$ in $90^{\circ}$

- bring steelball to the center again

- rotation of $\varphi$ in $180^{\circ}$ shows

misalignment of the beamcenter

(diagonal aberration from origin)

2. Determination of the new origin:

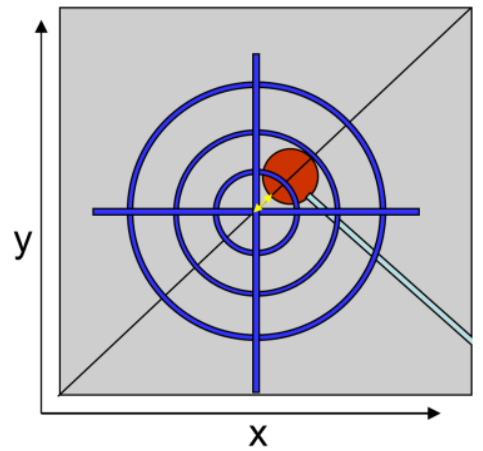

adjust the steelball half way to the center

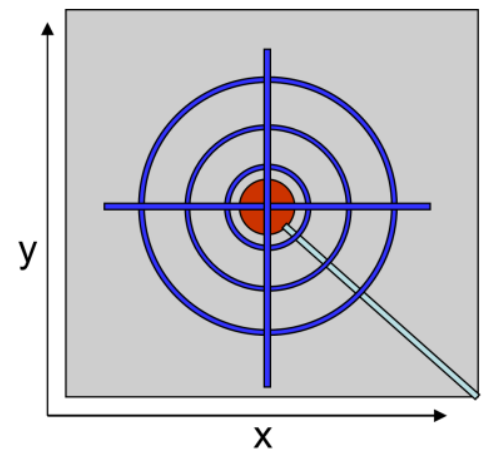

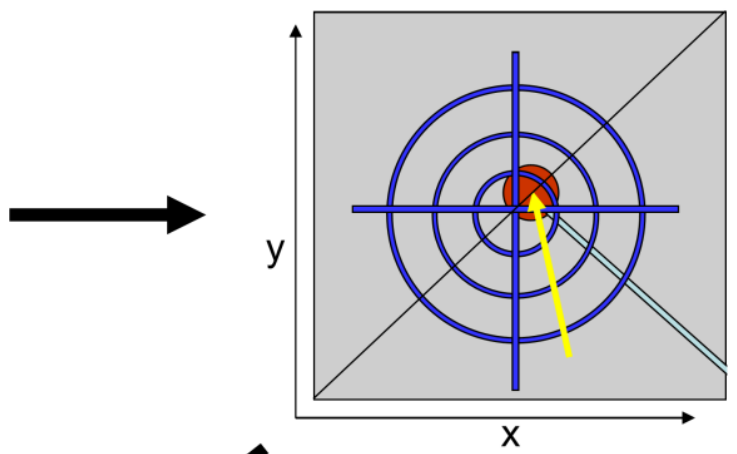

use tools --> options --> select origin to select the new origin in the center of the steelball.

(only in diagonal direction,

lower left to upper right)

repeat step 1.

Now the steelball should be centered at position 'right'.

(otherwise repeat step 2.) 
3. Drive to 'left' position to align the height.

the height is now either

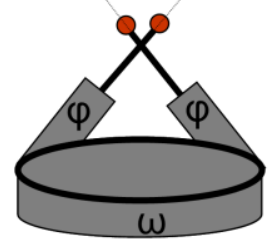

too high

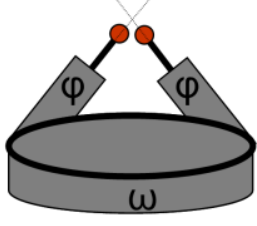

too low

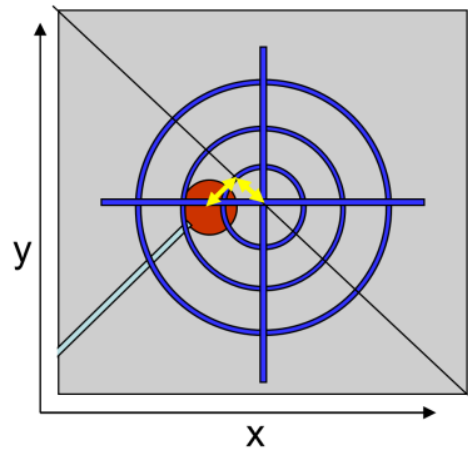

-adjust the height to position the center of the steell ball on the diagonal (upper left to lower right)

- set the origin along the diagonal to bring the steelball to the center

- check the centering by repeating step 1.

(it may be nessecary to repeat the whole procedure)

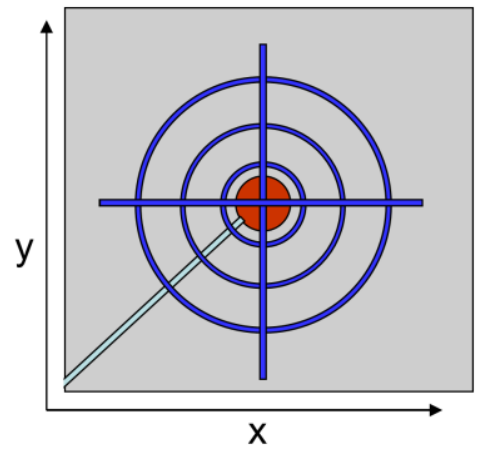




\subsection{Beam Stop Development}

For the helium cooled measurements, a new beam stop holder had to be developed. Otherwise, the standard Bruker beam stop holder would have been interfered with the crystal cooling device. The new beam stop holder holds the beam stop from horizontal position to free the space above. It was clamped on the rod of the regular nitrogen cooling device of the diffractometer with an annular sleeve. The distance from the stand to the beam stop pot is adjustable with a telescopic rod. The top of the clamp fixture for the beam stop sheet metal can be rotated to adjust its angle. The other two axes in space can be adjusted precisely by two screws at the clamp ring.
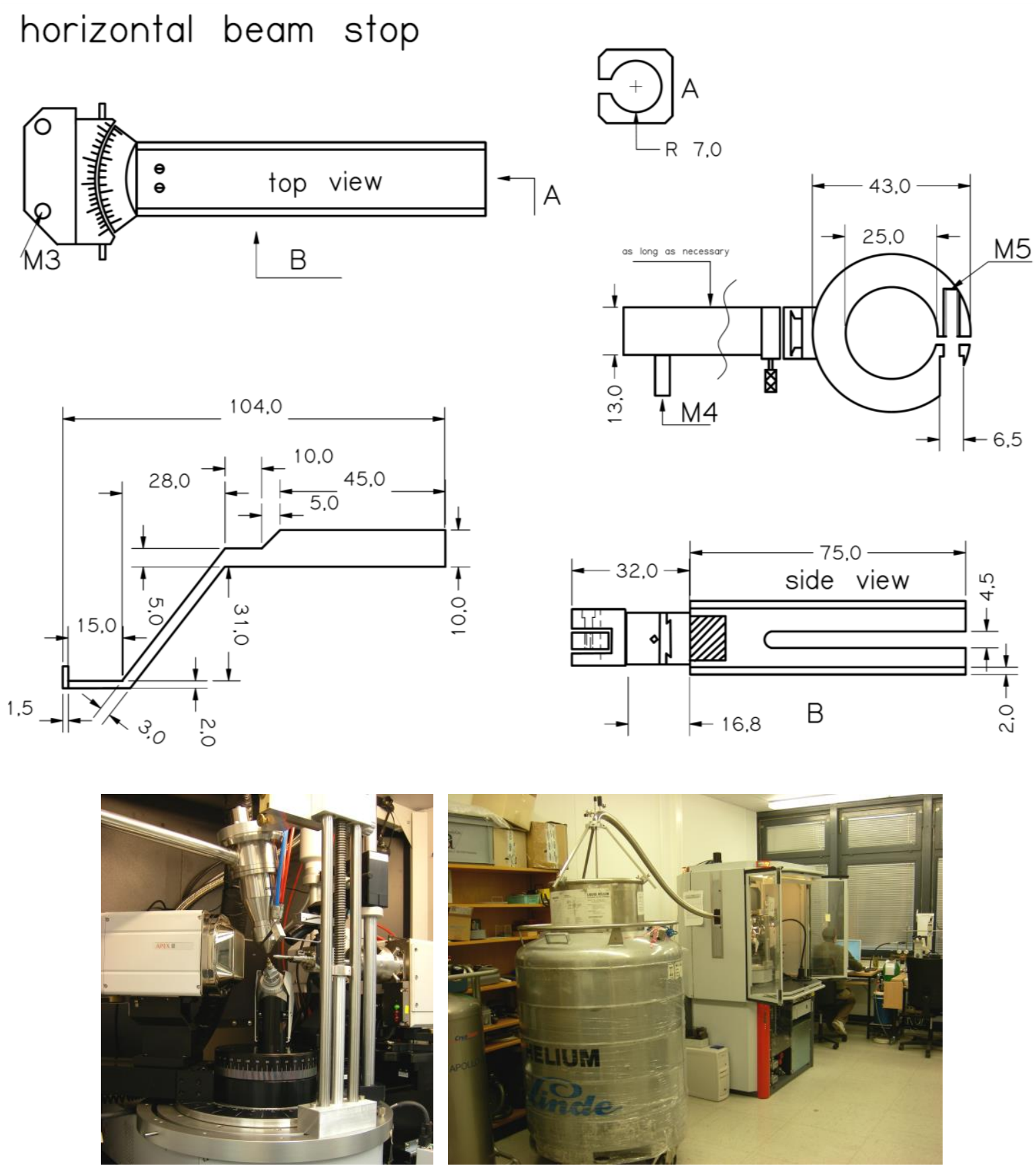

Figure 59 Measurement setup for helium-cooled single diffraction experiments on a Bruker TXS. 
For regular measurements the Bruker beam stop holder was also replaced by an easy adjustable vertical suspended beam stop holder. The vertical holder consists of an aluminum block screwed on the place of the regular beam stop. In front is an old goniometer head attached which hold a steel rod. On top of the steel rod is placed the clamp for the beam stop sheet with its lead pot. The alignment is done with a regular nut key for goniometer heads.

\section{vertical beam stop}
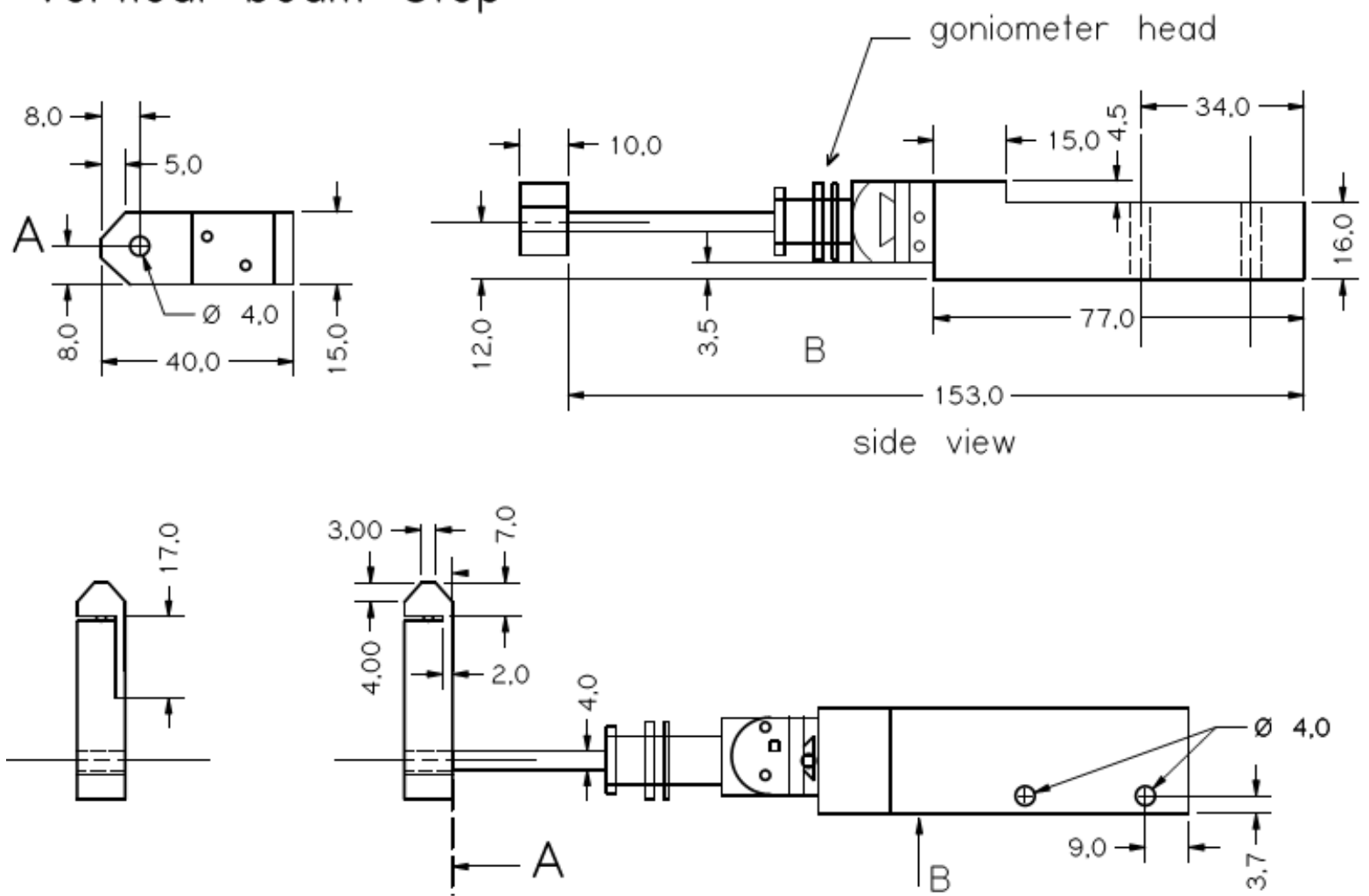

top view 


\subsection{Photo crystallography}

A LED array device suitable for the irradiation of single-crystals to induce photoreactions during the X-ray diffraction experiments was developed. A similar device was published by Raithby et al. ${ }^{[302]}$ but the present device has some improvements. The LED ring can be attached to Bruker Kryoflex or Agilent Cryojet cooling devices with a clamp ring. Three screws hold the ring in place while one other screw holds the LED ring in place. The six LEDs are connected to a powers source of $12 \mathrm{~V}$ which can be regulated from $4.4 \mathrm{~mA}$ to $32 \mathrm{~mA}$. The LEDS can also be exchanged to commercially available LASERs for pointing devices.

\section{Crystal Irradiation Device}
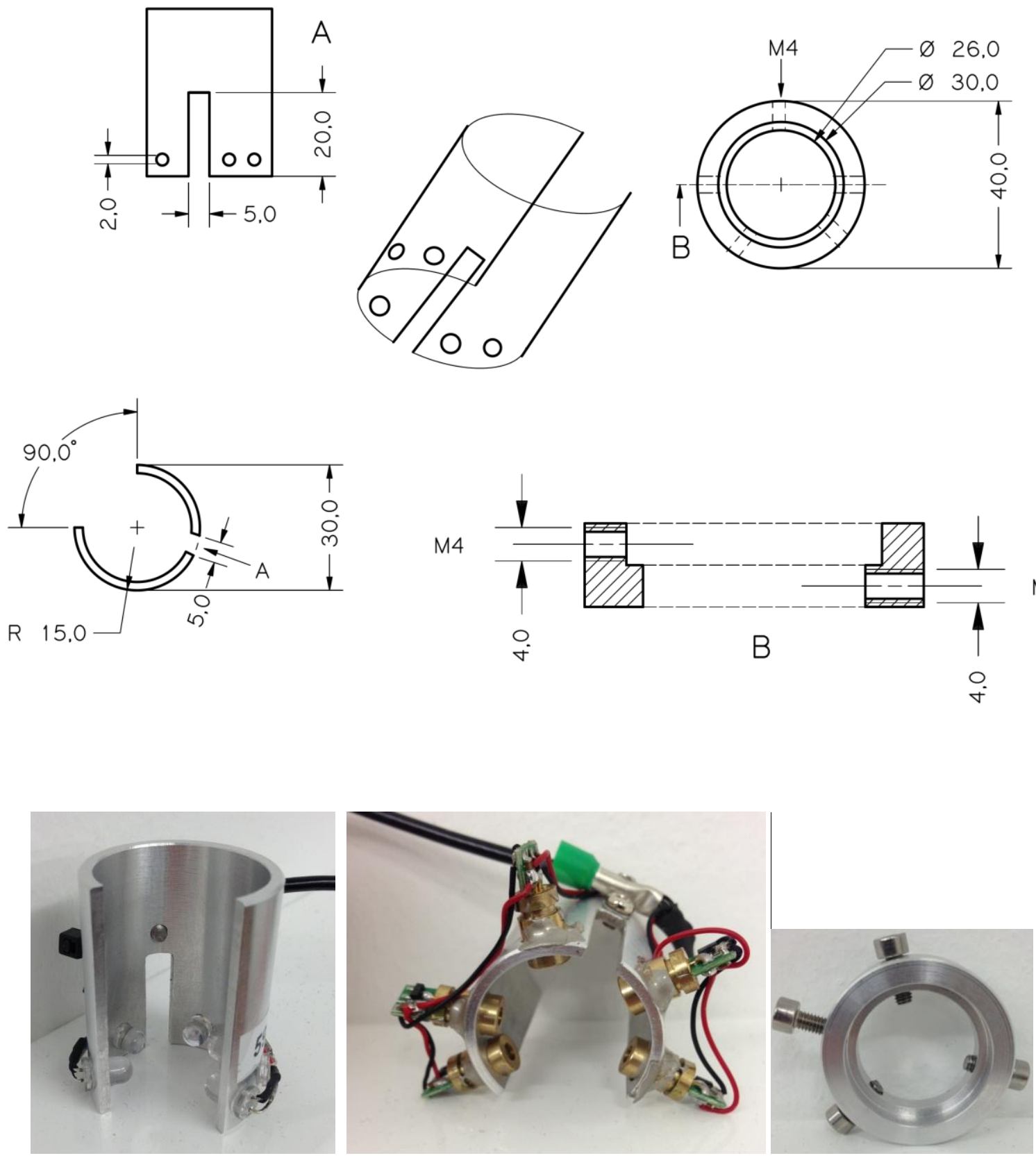

Figure 60 LED ring with regular high brilliant LEDs (left), with red LASERs (middle) and the clamp ring (right). 


\subsection{Parameters at Bond Critical Points}

10.4.1. Structure 1
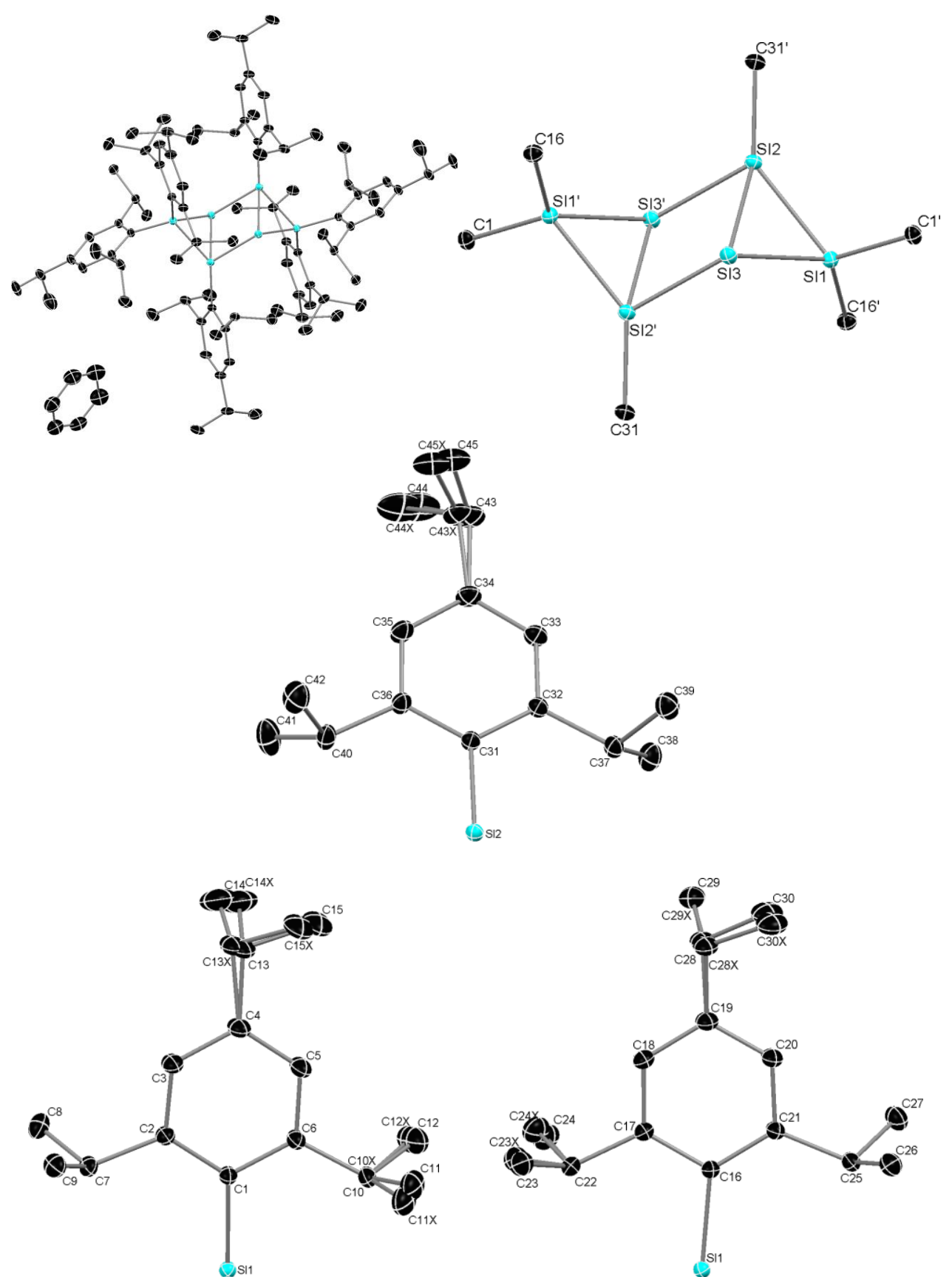

Table 17 Topological parameters of the bond critical points in 1 (BCPs, 3,-1) with esds calculated from XDPROP (only from ordered parts). 


\begin{tabular}{|c|c|c|c|c|c|c|c|c|c|}
\hline Bond & $\begin{array}{l}\rho\left(\mathbf{r}_{\mathrm{BCP}}\right) \\
{\left[\mathrm{e}^{-3}\right]}\end{array}$ & $\mid \begin{array}{l}\nabla^{2} \rho\left(\mathbf{r}_{\mathrm{BCP}}\right) \\
{\left[\mathrm{e}^{-5}\right]}\end{array}$ & $\begin{array}{l}d_{\mathrm{BP}} \\
{[\AA \AA]}\end{array}$ & \begin{tabular}{|l}
$d 1_{\mathrm{BCP}}$ \\
$[\AA]]$ \\
\end{tabular} & $\begin{array}{l}d 2_{\mathrm{BCP}} \\
[\AA]]\end{array}$ & $\begin{array}{l}\lambda_{1} \\
{\left[\mathrm{e} \AA^{-5}\right]}\end{array}$ & $\begin{array}{l}\lambda_{2} \\
{\left[\mathrm{e} \AA^{-5}\right]}\end{array}$ & $\begin{array}{l}\lambda_{3} \\
{\left[\mathrm{e} \AA^{-5}\right]}\end{array}$ & $\epsilon\left(\mathbf{r}_{\mathrm{BCP}}\right)$ \\
\hline SI(1)-SI(2) & $0.537(5)$ & $-1.645(8)$ & 2.3359 & 1.1215 & 1.2144 & -1.52 & -1.27 & 1.15 & 0.20 \\
\hline SI(1)-SI(3) & $0.545(6)$ & $-1.628(8)$ & 2.3676 & 1.1422 & 1.2254 & -1.54 & -1.28 & 1.20 & 0.21 \\
\hline SI(2)-SI(3) & $0.466(7)$ & $-1.164(9)$ & 2.3454 & 1.1855 & 1.1599 & -1.31 & -1.08 & 1.23 & 0.20 \\
\hline$S I(1)-C(1)$ & $0.826(7)$ & $2.315(17)$ & 1.9210 & 0.7398 & 1.1812 & -3.93 & -3.70 & 9.95 & 0.06 \\
\hline$S I(1)-C(16)$ & $0.853(7)$ & $2.354(17)$ & 1.9143 & 0.7346 & 1.1797 & -4.37 & -3.92 & 10.64 & 0.12 \\
\hline $\mathrm{SI}(2)-\mathrm{C}(31)$ & $0.776(8)$ & $2.288(19)$ & 1.8856 & 0.7454 & 1.1402 & -4.02 & -3.30 & 9.61 & 0.22 \\
\hline X3_SI(2)-SI(3) & $0.595(11)$ & $-2.285(12)$ & 2.3112 & 1.1282 & 1.1830 & -1.72 & -1.56 & 1.00 & 0.10 \\
\hline X3_SI(3)-SI(2) & $0.595(11)$ & $-2.284(12)$ & 2.3112 & 1.1828 & 1.1284 & -1.72 & -1.56 & 1.00 & 0.10 \\
\hline$C(1)-C(2)$ & $2.013(16)$ & $-16.044(49)$ & 1.4234 & 0.7149 & 0.7085 & -14.38 & -12.60 & 10.94 & 0.14 \\
\hline$C(1)-C(6)$ & $2.015(16)$ & $-15.285(51)$ & 1.4163 & 0.7051 & 0.7112 & -13.96 & -12.32 & 10.99 & 0.13 \\
\hline$C(7)-C(8)$ & $1.580(0)$ & $-9.401(0)$ & 1.5369 & 0.7727 & 0.7642 & -10.28 & -10.18 & 11.06 & 0.01 \\
\hline$C(7)-C(9)$ & $1.585(0)$ & $-9.493(0)$ & 1.5345 & 0.7714 & 0.7631 & -10.33 & -10.23 & 11.06 & 0.01 \\
\hline$C(16)-C(17)$ & $1.987(16)$ & $-15.136(52)$ & 1.4198 & 0.7004 & 0.7195 & -13.90 & -12.15 & 10.91 & 0.14 \\
\hline$C(16)-C(21)$ & $2.022(16)$ & $-15.548(49)$ & 1.4201 & 0.7178 & 0.7023 & -14.02 & -12.59 & 11.06 & 0.11 \\
\hline$C(20)-C(21)$ & $2.040(1)$ & $-15.948(0)$ & 1.4036 & 0.6979 & 0.7057 & -14.67 & -12.26 & 10.99 & 0.20 \\
\hline$C(21)-C(25)$ & $1.652(0)$ & $-10.748(0)$ & 1.5231 & 0.7757 & 0.7474 & -10.88 & -10.57 & 10.70 & 0.03 \\
\hline$C(25)-C(26)$ & $1.584(0)$ & $-9.467(0)$ & 1.5352 & 0.7718 & 0.7633 & -10.31 & -10.22 & 11.06 & 0.01 \\
\hline$C(25)-C(27)$ & $1.578(0)$ & $-9.382(0)$ & 1.5374 & 0.7730 & 0.7644 & -10.27 & -10.18 & 11.06 & 0.01 \\
\hline$C(31)-C(32)$ & $2.003(16)$ & $-15.032(50)$ & 1.4129 & 0.7068 & 0.7061 & -13.94 & -12.09 & 10.99 & 0.15 \\
\hline$C(31)-C(36)$ & $2.082(16)$ & $-17.089(48)$ & 1.4171 & 0.7237 & 0.6934 & -15.39 & -12.65 & 10.95 & 0.22 \\
\hline$C(32)-C(33)$ & $2.059(1)$ & $-16.365(0)$ & 1.3972 & 0.7026 & 0.6946 & -14.84 & -12.41 & 10.88 & 0.20 \\
\hline$C(32)-C(37)$ & $1.660(1)$ & $-10.946(0)$ & 1.5192 & 0.7733 & 0.7459 & -10.99 & -10.64 & 10.68 & 0.03 \\
\hline$C(33)-C(34)$ & $2.105(0)$ & $-16.866(0)$ & 1.3936 & 0.6950 & 0.6986 & -15.22 & -12.67 & 11.03 & 0.20 \\
\hline$C(33)-C(35)$ & $0.154(3)$ & $3.616(2)$ & 2.7746 & 1.3961 & 1.3785 & -0.46 & 1.97 & 2.10 & 0.00 \\
\hline$C(34)-C(35)$ & $2.080(0)$ & $-16.300(0)$ & 1.3964 & 0.6975 & 0.6989 & -14.91 & -12.49 & 11.10 & 0.19 \\
\hline$C(35)-C(36)$ & $2.057(1)$ & $-16.373(0)$ & 1.3972 & 0.6939 & 0.7032 & -14.87 & -12.40 & 10.90 & 0.20 \\
\hline$C(40)-C(42)$ & $1.596(0)$ & $-9.660(0)$ & 1.5299 & 0.7692 & 0.7608 & -10.41 & -10.31 & 11.06 & 0.01 \\
\hline$C(17)-C(18)$ & $2.070(1)$ & $-16.345(0)$ & 1.3963 & 0.7055 & 0.6908 & -14.85 & -12.45 & 10.96 & 0.19 \\
\hline$C(18)-C(19)$ & $2.093(0)$ & $-16.590(0)$ & 1.3920 & 0.6968 & 0.6952 & -15.03 & -12.59 & 11.03 & 0.19 \\
\hline$C(19)-C(20)$ & $2.117(0)$ & $-17.066(0)$ & 1.3904 & 0.6975 & 0.6929 & -15.29 & -12.75 & 10.98 & 0.20 \\
\hline$C(2)-C(3)$ & $2.048(1)$ & $-16.192(0)$ & 1.4002 & 0.7046 & 0.6956 & -14.79 & -12.34 & 10.93 & 0.20 \\
\hline$C(3)-C(4)$ & $2.085(0)$ & $-16.429(0)$ & 1.3944 & 0.6978 & 0.6966 & -14.96 & -12.53 & 11.06 & 0.19 \\
\hline$C(5)-C(6)$ & $2.059(1)$ & $-16.075(0)$ & 1.4003 & 0.6936 & 0.7067 & -14.74 & -12.36 & 11.03 & 0.19 \\
\hline$C(40)-C(41)$ & $1.595(0)$ & $-9.654(0)$ & 1.5302 & 0.7692 & 0.7610 & -10.41 & -10.30 & 11.06 & 0.01 \\
\hline$C(37)-C(38)$ & $1.591(0)$ & $-9.595(0)$ & 1.5319 & 0.7701 & 0.7617 & -10.38 & -10.28 & 11.06 & 0.01 \\
\hline$C(37)-C(39)$ & $1.591(0)$ & $-9.582(0)$ & 1.5322 & 0.7703 & 0.7619 & -10.37 & -10.27 & 11.06 & 0.01 \\
\hline$C(40)-C(36)$ & $1.665(1)$ & $-10.986(0)$ & 1.5179 & 0.7446 & 0.7733 & -11.02 & -10.67 & 10.70 & 0.03 \\
\hline$C(7)-C(2)$ & $1.654(0)$ & $-10.802(0)$ & 1.5222 & 0.7469 & 0.7753 & -10.91 & -10.59 & 10.70 & 0.03 \\
\hline$C(4)-C(5)$ & $2.115(0)$ & $-17.097(0)$ & 1.3903 & 0.6966 & 0.6937 & -15.31 & -12.75 & 10.96 & 0.20 \\
\hline
\end{tabular}

$\lambda$ : curvature of $\rho(\mathbf{r})$ at the $\mathrm{BCP}, d_{\mathrm{BP}}$ : The total length of the bond path, $d 1_{\mathrm{BCP}}$ : The distance of the first named atom to the BCP. 
10.4.2. Structure 2
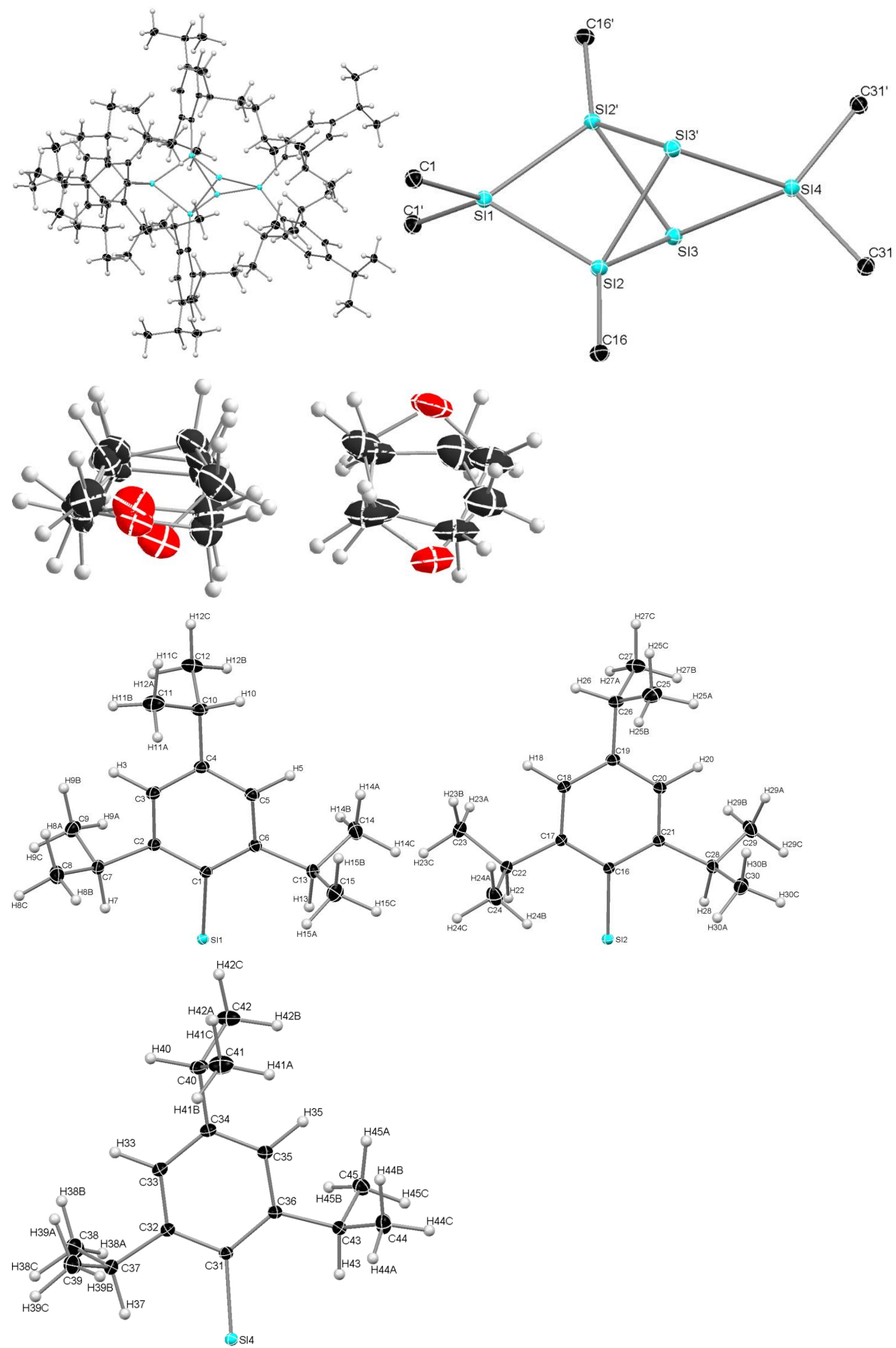

Table 18 Topological parameters of the bond critical points in $\mathbf{2}$ (BCPs, 3,-1) with esds calculated from XDPROP (only from ordered parts). 


\begin{tabular}{|c|c|c|c|c|c|c|c|c|c|}
\hline Bond & $\begin{array}{l}\rho\left(\mathrm{r}_{\mathrm{BCP}}\right) \\
{\left[\mathrm{e}^{-3}{ }^{-3}\right]} \\
\end{array}$ & $\begin{array}{l}\nabla^{2} \rho\left(\mathbf{r}_{\mathrm{BCP}}\right) \\
{\left[\mathrm{e} \AA^{-5}\right]} \\
\end{array}$ & $\begin{array}{l}d_{\mathrm{BP}} \\
{[\AA \AA]}\end{array}$ & $\begin{array}{l}d 1_{\mathrm{BCP}} \\
[\AA]] \\
\end{array}$ & $\begin{array}{l}d 2_{\mathrm{BCP}} \\
[\AA]] \\
\end{array}$ & $\begin{array}{l}\lambda_{1} \\
{\left[\mathrm{e} \AA^{-5}\right]}\end{array}$ & $\begin{array}{l}\lambda_{2} \\
{\left[\mathrm{e} \AA^{-5}\right]} \\
\end{array}$ & $\begin{array}{l}\lambda_{3} \\
{\left[\mathrm{e}^{-5}\right]}\end{array}$ & $\epsilon\left(\mathrm{r}_{\mathrm{BCP}}\right)$ \\
\hline SI(1)-SI(2) & $0.580(5)$ & $-2.942(8)$ & 2.3862 & 1.3052 & 1.0810 & -1.95 & -1.74 & 0.75 & 0.13 \\
\hline SI(1)-X2_SI(2) & $0.580(6)$ & $-2.941(8)$ & 2.3862 & 1.3051 & 1.0811 & -1.96 & -1.74 & 0.75 & 0.13 \\
\hline SI(2)-SI(3) & $0.512(4)$ & $-1.586(7)$ & 2.3620 & 1.2505 & 1.1115 & -1.60 & -1.07 & 1.09 & 0.49 \\
\hline SI(2)-X2_SI(3) & $0.555(8)$ & $-1.893(8)$ & 2.3808 & 1.2603 & 1.1205 & -1.80 & -1.10 & 1.00 & 0.64 \\
\hline X2_SI(2)-X2_SI(3) & $0.512(6)$ & $-1.586(8)$ & 2.3619 & 1.2505 & 1.1115 & -1.60 & -1.08 & 1.09 & 0.49 \\
\hline SI(3)-SI(4) & $0.527(4)$ & $-1.925(4)$ & 2.4113 & 1.2166 & 1.1947 & -1.73 & -1.25 & 1.06 & 0.38 \\
\hline SI(3)-X2_SI(2) & $0.554(9)$ & $-1.895(10)$ & 2.3808 & 1.1204 & 1.2604 & -1.80 & -1.10 & 1.00 & 0.64 \\
\hline SI(4)-X2_SI(3) & $0.527(5)$ & $-1.925(7)$ & 2.4113 & 1.1945 & 1.2167 & -1.73 & -1.25 & 1.06 & 0.38 \\
\hline$S I(1)-C(1)$ & $0.771(5)$ & $2.822(8)$ & 1.9208 & 0.7425 & 1.1782 & -3.59 & -3.47 & 9.88 & 0.04 \\
\hline $\mathrm{SI}(1)-\mathrm{X} 2 \_\mathrm{C}(1)$ & $0.766(5)$ & $2.556(9)$ & 1.9208 & 0.7444 & 1.1765 & -3.56 & -3.45 & 9.57 & 0.03 \\
\hline$S I(2)-C(16)$ & $0.776(7)$ & $2.645(16)$ & 1.8928 & 0.7442 & 1.1486 & -3.98 & -3.22 & 9.84 & 0.24 \\
\hline SI(4)-C(31) & $0.775(5)$ & $1.569(8)$ & 1.9236 & 0.7450 & 1.1787 & -4.22 & -3.63 & 9.42 & 0.16 \\
\hline SI(4)-X2_C(31) & $0.770(6)$ & $1.268(9)$ & 1.9237 & 0.7471 & 1.1765 & -4.19 & -3.60 & 9.06 & 0.16 \\
\hline X2_SI(2)-X2_C(16) & $0.770(8)$ & $2.309(15)$ & 1.8928 & 0.7465 & 1.1462 & -3.95 & -3.19 & 9.44 & 0.24 \\
\hline$C(1)-C(2)$ & $2.012(14)$ & $-15.812(44)$ & 1.4228 & 0.7175 & 0.7052 & -14.46 & -12.46 & 11.11 & 0.16 \\
\hline$C(1)-C(6)$ & $2.038(16)$ & $-16.385(54)$ & 1.4178 & 0.6744 & 0.7434 & -15.63 & -12.69 & 11.94 & 0.23 \\
\hline$C(2)-C(3)$ & $2.049(1)$ & $-16.468(0)$ & 1.3968 & 0.6979 & 0.6990 & -14.82 & -12.39 & 10.74 & 0.20 \\
\hline$C(2)-C(7)$ & $1.631(0)$ & $-10.573(0)$ & 1.5264 & 0.7737 & 0.7528 & -10.72 & -10.45 & 10.60 & 0.03 \\
\hline$C(3)-C(4)$ & $2.109(0)$ & $-17.168(0)$ & 1.3924 & 0.6968 & 0.6956 & -15.33 & -12.75 & 10.91 & 0.20 \\
\hline$C(3)-C(5)$ & $0.148(2)$ & $3.476(2)$ & 2.7778 & 1.3999 & 1.3779 & -0.43 & 1.89 & 2.02 & 0.00 \\
\hline$C(4)-C(5)$ & $2.116(0)$ & $-17.516(0)$ & 1.3915 & 0.6967 & 0.6948 & -15.50 & -12.87 & 10.86 & 0.20 \\
\hline$C(4)-C(10)$ & $1.673(0)$ & $-11.181(0)$ & 1.5139 & 0.7748 & 0.7391 & -11.07 & -10.74 & 10.64 & 0.03 \\
\hline$C(5)-C(6)$ & $2.102(0)$ & $-17.418(0)$ & 1.3996 & 0.6692 & 0.7305 & -16.00 & -13.15 & 11.73 & 0.22 \\
\hline$C(6)-C(13)$ & $1.684(0)$ & $-11.472(0)$ & 1.5210 & 0.7931 & 0.7279 & -11.54 & -11.17 & 11.24 & 0.03 \\
\hline$C(7)-C(8)$ & $1.579(0)$ & $-9.370(0)$ & 1.5361 & 0.7738 & 0.7624 & -10.31 & -10.17 & 11.11 & 0.01 \\
\hline$C(7)-C(9)$ & $1.577(0)$ & $-9.358(0)$ & 1.5370 & 0.7744 & 0.7626 & -10.29 & -10.17 & 11.10 & 0.01 \\
\hline$C(10-C(11)$ & $1.584(0)$ & $-9.613(0)$ & 1.5302 & 0.7711 & 0.7591 & -10.36 & -10.25 & 11.00 & 0.01 \\
\hline$C(10-C(12)$ & $1.578(0)$ & $-9.529(0)$ & 1.5323 & 0.7725 & 0.7599 & -10.33 & -10.20 & 11.00 & 0.01 \\
\hline$C(13)-C(14)$ & $1.580(0)$ & $-9.450(0)$ & 1.5342 & 0.7722 & 0.7620 & -10.33 & -10.21 & 11.09 & 0.01 \\
\hline$C(13)-C(15)$ & $1.594(0)$ & $-9.648(0)$ & 1.5291 & 0.7697 & 0.7594 & -10.44 & -10.30 & 11.09 & 0.01 \\
\hline$C(16)-C(17)$ & $1.995(5)$ & $-15.876(48)$ & 1.4190 & 0.7038 & 0.7152 & -14.61 & -12.25 & 10.99 & 0.19 \\
\hline$C(16)-C(21)$ & $2.014(5)$ & $-16.216(45)$ & 1.4212 & 0.7100 & 0.7112 & -14.73 & -12.51 & 11.02 & 0.18 \\
\hline$C(17)-C(18)$ & $2.040(0)$ & $-16.267(0)$ & 1.3999 & 0.7026 & 0.6973 & -14.77 & -12.31 & 10.81 & 0.20 \\
\hline$C(17)-C(22)$ & $1.648(0)$ & $-10.824(0)$ & 1.5221 & 0.7727 & 0.7494 & -10.86 & -10.58 & 10.62 & 0.03 \\
\hline$C(18)-C(19)$ & $2.093(0)$ & $-17.033(0)$ & 1.3941 & 0.6978 & 0.6964 & -15.24 & -12.66 & 10.87 & 0.20 \\
\hline$C(18)-C(20)$ & $0.134(3)$ & $3.629(2)$ & 2.8004 & 1.4056 & 1.3948 & -0.34 & 1.87 & 2.10 & 0.00 \\
\hline$C(19)-C(20)$ & $2.103(0)$ & $-17.303(0)$ & 1.3944 & 0.6975 & 0.6969 & -15.39 & -12.77 & 10.86 & 0.20 \\
\hline$C(19)-C(26)$ & $1.669(0)$ & $-11.161(0)$ & 1.5139 & 0.7736 & 0.7404 & -11.04 & -10.73 & 10.61 & 0.03 \\
\hline$C(20)-C(21)$ & $2.055(1)$ & $-16.638(0)$ & 1.3944 & 0.6945 & 0.6999 & -14.92 & -12.44 & 10.72 & 0.20 \\
\hline$C(21)-C(28)$ & $1.643(0)$ & $-10.728(0)$ & 1.5240 & 0.7740 & 0.7499 & -10.81 & -10.54 & 10.63 & 0.03 \\
\hline$C(22)-C(23)$ & $1.594(0)$ & $-9.629(0)$ & 1.5290 & 0.7706 & 0.7583 & -10.43 & -10.30 & 11.10 & 0.01 \\
\hline$C(22)-C(24)$ & $1.598(0)$ & $-9.672(0)$ & 1.5286 & 0.7704 & 0.7582 & -10.45 & -10.32 & 11.09 & 0.01 \\
\hline$C(25)-C(26)$ & $1.582(0)$ & $-9.623(0)$ & 1.5305 & 0.7591 & 0.7714 & -10.36 & -10.26 & 10.99 & 0.01 \\
\hline$C(26)-C(27)$ & $1.580(0)$ & $-9.586(0)$ & 1.5313 & 0.7719 & 0.7594 & -10.35 & -10.23 & 10.99 & 0.01 \\
\hline$C(28)-C(29)$ & $1.582(0)$ & $-9.479(0)$ & 1.5336 & 0.7731 & 0.7604 & -10.35 & -10.22 & 11.09 & 0.01 \\
\hline$C(28)-C(30)$ & $1.587(0)$ & $-9.519(0)$ & 1.5327 & 0.7726 & 0.7601 & -10.36 & -10.24 & 11.09 & 0.01 \\
\hline$C(31)-C(32)$ & $2.005(5)$ & $-15.770(46)$ & 1.4197 & 0.7049 & 0.7149 & -14.45 & -12.41 & 11.09 & 0.16 \\
\hline$C(31)-C(36)$ & $2.011(5)$ & $-16.237(49)$ & 1.4224 & 0.7063 & 0.7161 & -14.63 & -12.65 & 11.04 & 0.16 \\
\hline$C(32)-C(33)$ & $2.056(1)$ & $-16.430(0)$ & 1.3975 & 0.7032 & 0.6944 & -14.86 & -12.41 & 10.84 & 0.20 \\
\hline$C(32)-C(37)$ & $1.660(0)$ & $-10.996(0)$ & 1.5173 & 0.7736 & 0.7437 & -10.95 & -10.66 & 10.62 & 0.03 \\
\hline$C(33)-C(34)$ & $2.108(0)$ & $-17.363(0)$ & 1.3894 & 0.6967 & 0.6926 & -15.36 & -12.78 & 10.77 & 0.20 \\
\hline$C(33)-C(35)$ & $0.142(2)$ & $3.619(2)$ & 2.7778 & 1.3996 & 1.3782 & -0.40 & 1.87 & 2.15 & 0.00 \\
\hline$C(34)-C(35)$ & $2.102(0)$ & $-17.302(0)$ & 1.3942 & 0.6963 & 0.6979 & -15.38 & -12.77 & 10.85 & 0.20 \\
\hline$C(34)-C(40)$ & $1.665(0)$ & $-11.116(0)$ & 1.5146 & 0.7732 & 0.7414 & -11.02 & -10.70 & 10.60 & 0.03 \\
\hline$C(35)-C(36)$ & $2.048(0)$ & $-16.287(0)$ & 1.3999 & 0.6955 & 0.7044 & -14.82 & -12.36 & 10.89 & 0.20 \\
\hline$C(36)-C(43)$ & $1.642(0)$ & $-10.646(0)$ & 1.5250 & 0.7776 & 0.7474 & -10.77 & -10.53 & 10.65 & 0.02 \\
\hline$C(37)-C(38)$ & $1.586(0)$ & $-9.521(0)$ & 1.5322 & 0.7706 & 0.7616 & -10.37 & -10.23 & 11.08 & 0.01 \\
\hline$C(37)-C(39)$ & $1.592(0)$ & $-9.631(0)$ & 1.5300 & 0.7693 & 0.7607 & -10.41 & -10.29 & 11.08 & 0.01 \\
\hline
\end{tabular}




\begin{tabular}{l|l|l|l|l|l|r|r|r|r}
$\mathrm{C}(40)-\mathrm{C}(41)$ & $1.570(0)$ & $-9.402(0)$ & 1.5357 & 0.7738 & 0.7619 & -10.25 & -10.15 & 11.00 & 0.01 \\
$\mathrm{C}(40)-\mathrm{C}(42)$ & $1.583(0)$ & $-9.637(0)$ & 1.5295 & 0.7707 & 0.7588 & -10.39 & -10.24 & 10.99 & 0.01 \\
$\mathrm{C}(43)-\mathrm{C}(44)$ & $1.569(0)$ & $-9.260(0)$ & 1.5390 & 0.7738 & 0.7651 & -10.23 & -10.12 & 11.09 & 0.01 \\
$\mathrm{SI}(3)-\mathrm{X} 2 \mathrm{SI}(3)$ & $0.322(4)$ & $0.964(4)$ & 2.7393 & 1.3696 & 1.3696 & -0.57 & 0.44 & 1.10 & 0.00
\end{tabular}

$\lambda$ : curvature of $\rho(r)$ at the $\mathrm{BCP}, d_{\mathrm{BP}}$ : The total length of the bond path, $d 1_{\mathrm{BCP}}$ : The distance of the first named atom to the BCP.

\subsection{Bond Lengths and Angles}

\subsubsection{Structure 3}

\begin{tabular}{|c|c|c|c|c|c|}
\hline \multicolumn{2}{|c|}{ bond length $[\AA]]$} & \multicolumn{2}{|l|}{ angles $\left[^{\circ}\right]$} & \multicolumn{2}{|l|}{ angles $\left[{ }^{\circ}\right]$} \\
\hline$N(1)-N(2)$ & $1.3755(2)$ & $N(2)-N(1)-C(1)$ & 105.971(17) & $H(4 A)-C(4)-H(4 B)$ & 109.480376(0) \\
\hline$N(1)-C(1)$ & $1.3359(3)$ & $N(1)-N(2)-C(3)$ & $110.941(18)$ & $H(4 A)-C(4)-H(4 C)$ & 109.475933(0) \\
\hline$N(2)-C(3)$ & $1.3532(2)$ & $N(1)-N(2)-C(6)$ & 123.443(19) & $H(4 A)-C(4)-H(4 D)$ & $134.710806(0)$ \\
\hline$N(2)-C(6)$ & $1.4421(2)$ & $N(1)-N(2)-C(6)$ & 123.444(19) & $H(4 A)-C(4)-H(4 E)$ & $34.262317(0)$ \\
\hline$N(2)-C(6)$ & $1.4420(2)$ & $N(1)-N(2)-C(6)$ & $123.444(19)$ & $H(4 A)-C(4)-H(4 F)$ & $77.879056(0)$ \\
\hline$N(2)-C(6)$ & $1.4421(2)$ & $C(3)-N(2)-C(6)$ & 125.609(19) & $\mathrm{H}(4 \mathrm{~B})-\mathrm{C}(4)-\mathrm{H}(4 \mathrm{C})$ & $109.474505(0)$ \\
\hline$C(1)-C(2)$ & $1.4076(3)$ & $C(3)-N(2)-C(6)$ & $125.608(19)$ & $H(4 B)-C(4)-H(4 D)$ & $77.864064(0)$ \\
\hline$C(1)-C(4)$ & $1.4918(3)$ & $C(3)-N(2)-C(6)$ & $125.608(19)$ & $\mathrm{H}(4 \mathrm{~B})-\mathrm{C}(4)-\mathrm{H}(4 \mathrm{E})$ & $134.720564(0)$ \\
\hline$C(2)-C(3)$ & $1.3865(3)$ & $C(6)-N(2)-C(6)$ & $0.001818(1)$ & $\mathrm{H}(4 \mathrm{~B})-\mathrm{C}(4)-\mathrm{H}(4 \mathrm{~F})$ & $34.247484(0)$ \\
\hline$C(2)-H(2)$ & $1.075604(0)$ & $C(6)-N(2)-C(6)$ & $0.004119(1)$ & $H(4 C)-C(4)-H(4 D)$ & $34.254704(0)$ \\
\hline$C(3)-C(5)$ & $1.4869(3)$ & $C(6)-N(2)-C(6)$ & $0.003096(0)$ & $\mathrm{H}(4 \mathrm{C})-\mathrm{C}(4)-\mathrm{H}(4 \mathrm{E})$ & $77.859772(0)$ \\
\hline$C(4)-H(4 A)$ & $1.084232(0)$ & $N(1)-C(1)-C(2)$ & $110.318(18)$ & $\mathrm{H}(4 \mathrm{C})-\mathrm{C}(4)-\mathrm{H}(4 \mathrm{~F})$ & $134.709174(0)$ \\
\hline $\mathrm{C}(4)-\mathrm{H}(4 \mathrm{~B})$ & $1.084585(0)$ & $N(1)-C(1)-C(4)$ & $121.541(17)$ & $H(4 D)-C(4)-H(4 E)$ & $109.468630(0)$ \\
\hline $\mathrm{C}(4)-\mathrm{H}(4 \mathrm{C})$ & $1.084588(0)$ & $C(2)-C(1)-C(4)$ & $128.137(17)$ & $\mathrm{H}(4 \mathrm{D})-\mathrm{C}(4)-\mathrm{H}(4 \mathrm{~F})$ & $109.466901(0)$ \\
\hline$C(4)-H(4 D)$ & $1.084597(0)$ & $C(1)-C(2)-C(3)$ & 105.695(17) & $\mathrm{H}(4 \mathrm{E})-\mathrm{C}(4)-\mathrm{H}(4 \mathrm{~F})$ & $109.494254(0)$ \\
\hline $\mathrm{C}(4)-\mathrm{H}(4 \mathrm{E})$ & $1.084348(0)$ & $C(1)-C(2)-H(2)$ & $126.101(11)$ & $C(3)-C(5)-H(5 A)$ & $111.092(9)$ \\
\hline $\mathrm{C}(4)-\mathrm{H}(4 \mathrm{~F})$ & $1.084543(0)$ & $C(3)-C(2)-H(2)$ & $128.203(12)$ & $C(3)-C(5)-H(5 B)$ & $108.900(9)$ \\
\hline$C(5)-H(5 A)$ & $1.084606(0)$ & $N(2)-C(3)-C(2)$ & 107.059(17) & $C(3)-C(5)-H(5 C)$ & $111.396(10)$ \\
\hline$C(5)-H(5 B)$ & $1.084407(0)$ & $N(2)-C(3)-C(5)$ & $122.039(17)$ & $H(5 A)-C(5)-H(5 B)$ & $110.500789(0)$ \\
\hline $\mathrm{C}(5)-\mathrm{H}(5 \mathrm{C})$ & $1.084411(0)$ & $C(2)-C(3)-C(5)$ & $130.862(17)$ & $H(5 A)-C(5)-H(5 C)$ & $106.346818(0)$ \\
\hline $\mathrm{H}(4 \mathrm{~A})-\mathrm{H}(4 \mathrm{E})$ & $0.638777(0)$ & $C(1)-C(4)-H(4 A)$ & $109.434(10)$ & $H(5 B)-C(5)-H(5 C)$ & $108.564565(0)$ \\
\hline $\mathrm{H}(4 \mathrm{~B})-\mathrm{H}(4 \mathrm{~F})$ & $0.638670(0)$ & $C(1)-C(4)-H(4 B)$ & $109.460(10)$ & $N(2)-C(6)-N(2)$ & 109.097(14) \\
\hline \multirow[t]{8}{*}{$H(4 C)-H(4 D)$} & $0.638818(0)$ & $C(1)-C(4)-H(4 C)$ & $109.502(10)$ & $N(2)-C(6)-N(2)$ & 109.101(14) \\
\hline & & $C(1)-C(4)-H(4 D)$ & $109.507(10)$ & $N(2)-C(6)-N(2)$ & 109.102(14) \\
\hline & & $C(1)-C(4)-H(4 E)$ & $109.454(10)$ & $\mathrm{C}(4)-\mathrm{H}(4 \mathrm{~A})-\mathrm{H}(4 \mathrm{E})$ & $72.878780(0)$ \\
\hline & & $\mathrm{C}(1)-\mathrm{C}(4)-\mathrm{H}(4 \mathrm{~F})$ & $109.437(10)$ & $\mathrm{C}(4)-\mathrm{H}(4 \mathrm{~B})-\mathrm{H}(4 \mathrm{~F})$ & $72.872659(0)$ \\
\hline & & & & $\mathrm{C}(4)-\mathrm{H}(4 \mathrm{C})-\mathrm{H}(4 \mathrm{D})$ & $72.873454(0)$ \\
\hline & & & & $\mathrm{C}(4)-\mathrm{H}(4 \mathrm{D})-\mathrm{H}(4 \mathrm{C})$ & $72.871844(0)$ \\
\hline & & & & $\mathrm{C}(4)-\mathrm{H}(4 \mathrm{E})-\mathrm{H}(4 \mathrm{~A})$ & $72.858905(0)$ \\
\hline & & & & $C(4)-H(4 F)-H(4 B)$ & $72.879859(0)$ \\
\hline
\end{tabular}


10.5.2. Atom Names of the Calculated Structure of 1

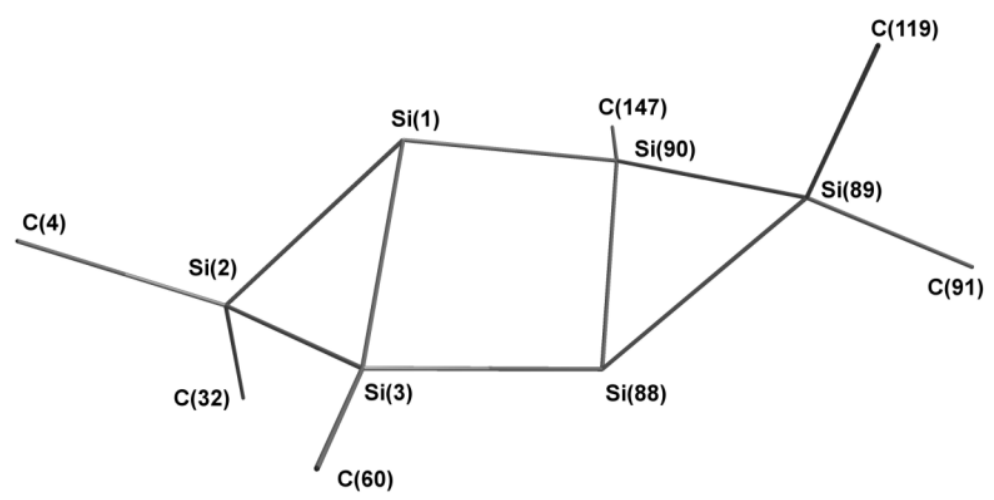

10.5.3. Atom names of the calculated structure of 2

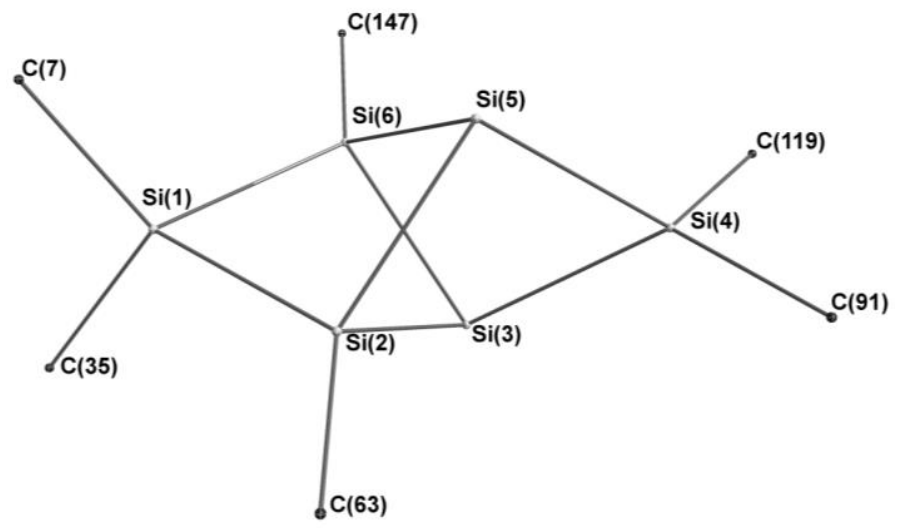




\section{Curriculum vitae}

Daniel Kratzert

Geboren, 05.01.1980 in Friedrichshafen

Staatsangehörigkeit: Deutsch

Groner Straße 21

37073 Göttingen

E-Mail: dkratzert@gmx.de

\section{Wissenschaftlicher Werdegang}

06.2008 - 04.2013 Promotion zum Thema "Charge Density Distribution in Low-Valent Tetreles" in der Arbeitsgruppe von Prof. Dr. Dietmar Stalke.

05.2008

Diplomprüfung an der Georg-August-Universität Göttingen.

05.2007-02.2008 Diplomarbeit zum Thema " $\mathrm{C}_{3}$-Symmetrische Iminophosphoranliganden" in der Arbeitsgruppe von Prof. Dr. D. Stalke. Note: "Sehr gut"

$10.2001-03.2007$ Studium der Chemie an der Georg-August-Universität Göttingen.

$08.2000-07.2001$ Zivildienst an der Alexander-Schmorell Schule in Kassel. Sonderschule für körperbehinderte Kinder.

$08.1999-06.2000$ Ausbildung zum Staatlich geprüften Chemisch-Technischen Assistenten an der Elisabeth-Knipping-Schule in Kassel.

06.1999

Allgemeine Hochschulreife, Elisabeth-Knipping Schule in Kassel.

\section{Teilnahme an wissenschaftlichen Tagungen}

03.2012

09.2011

09.2011

08.2011

09.2010

07.2010

04.2010

09.2009
Tutor beim "Workshop on Problem Structures" in Taipeh, Taiwan.

Besuch des Wissenschaftsforum der GDCh in Bremen.

"International School on Charge Density" in Jaca, Spanien.

Vortrag beim "XXII Congress and General Assembly of the International Union of Crystallography" in Madrid über "Germylenes: High resolution X-ray data for unambiguous ligand identification".

Teilnahme am "Bruker Users Group Meeting" in Karlsruhe.

Poster über "Coordination and Reactivity of $\left[\mathrm{Mg}\left(\mathrm{Pz}_{3}{ }_{3} \mathrm{C}\right)_{2}\right]$ " auf der "Gordon Research Conference" in Mount Holyoke, USA.

Vortrag beim CMC-Meeting in Aarhus über "Charge Density Quality Data". Teilnahme am ChemKrist-Workshop in Freiburg. 
Vortrag auf der DGK-Jahrestagung über "Experimentelle Elektronendichteuntersuchung an $\left[\mathrm{Mg}\left(\mathrm{Pz}_{3}{ }_{3} \mathrm{C}\right)_{2}\right]$ mit $15 \mathrm{~K}$-Daten".

Zwischenbericht des DFG Schwerpunktprogramms 1178 im Kloster Banz.

06.2008 Besuch des " $5^{\text {th }}$ European Charge Density Meeting" in Gravedona, Italien.

10.2007 SHELX Workshop und "Bruker Users Group Meeting" in Göttingen.

\section{Universitäre Tätigkeiten}

03.10

Seit 2009

Seit 06.2008

Seit 2008

Seit 02.06
Erlangung der Fachkunde im Strahlenschutz.

Verantwortlich für die Wartung der Einkristalldiffraktometer (Bruker Ag/Mo Microfocus Smart APEXII, Mo-Drehanode APEXII Ultra und Cu-Smart 6000).

Betreuung von Praktika und Seminaren für Studierende mit Hauptfach Chemie.

Durchführung von Service-Kristallstrukturanalyse.

Systemadministrator in der Arbeitsgruppe von Prof. Dr. D. Stalke.

\section{Persönliche Fähigkeiten}

Sprachen

Deutsch, Muttersprache.

Englisch, fließend in Wort und Schrift.

Französisch, Grundkenntnisse.

EDV

Fortgeschrittene Kenntnisse in der Linux- und Windows-

NetzwerkAdministration.

Fortgeschrittene Kenntnisse in kristallographischen Programmen (Bruker APEXII, SHELXTL Paket, ShelXle, Mercury, PLATON, WinGX, XShell, MoleCoolQT, XD2006, MoPro).

Fortgeschrittene Kenntnisse in den üblichen Office-Anwendungen (MS Word, Excel, Openoffice, Adobe Photoshop, PovRay, Chemdraw, Origin).

Grundkenntnisse in 2D CAD.

Programmiersprachen

Python, fortgeschrittene Kenntnisse.

C/C++, Grundkenntnisse.

Fortran, Grundkenntnisse. 


\section{Publikationen}

[22] "Donor-acceptor cyclopropanes with Lawesson's and Woollins' reagents: formation of bisthiophenes and unprecedented cage-like molecules" J. Kaschel, C. D. Schmidt, M. Mumby, D. Kratzert, D. Stalke, D. B. Werz, Chem. Comm. 2013, 49, 4403-4405.

[21] "An Experimental Charge Density Study of Two Isomers of Hexasilabenzene", D. Kratzert, D. Leusser, J. Holstein, B. Dittrich, K. Abersfelder, D. Scheschkewitz, D. Stalke, Angew. Chem Int.Ed. 2013, 52, 4478-4482.

[20] "Conversion of a Singlet Silylene to a stable Biradical", K. C. Mondal, H. W. Roesky, M. C. Schwarzer, G. Frenking, S. Neudeck, I. Tkach, H. Wolf, D. Kratzert, R. Herbst-Irmer, B. Niepötter, D. Stalke, Angew. Chem. Int. Ed. 2013, 52, 1801-1805.

[19] "Anharmonic Motion in Experimental Charge Density Investigations", R. Herbst-Irmer, J. Henn, J. J. Holstein, C. B. Huebschle, B. Dittrich, D. Stern, D. Kratzert, D. Stalke, J. Phys. Chem. 2013, A117, 633-641.

[18] "Domino Reactions of Donor-Acceptor-Substituted Cyclopropanes for the Synthesis of 3,3'Linked Oligopyrroles and Pyrrolo[3,2-e]indoles", J. Kaschel, T. F. Schneider, D. Kratzert, D. Stalke, D. B. Werz, Angew. Chem. Int. Ed. 2012, 51, 11153-11156.

[17] "Phenalenyl-Based Molecules: Tuning the Lowest Unoccupied Molecular Orbital to Design a Catalyst", T. K. Sen, A. Mukherjee, A. Modak, P. K. Ghorai, D. Kratzert, M. Granitzka, D. Stalke, S. K. Mandal, Chem. Eur. J. 2012, 18, 54-58.

[16] "A Remarkable End-On Activation of Diazoalkane and Cleavage of Both C-Cl Bonds of Dichloromethane with a Silylene to a Single Product with Five-Coordinate Silicon Atoms", S. S. Sen, J. Hey, D. Kratzert, H. W. Roesky, D. Stalke, Organometallics 2012, 31, 435-439.

[15] "Zwitterionic Si-C-Si-P and Si-P-Si-P Four-Membered Rings with Two-Coordinate Phosphorus Atoms", S. S. Sen, S. Khan, H. W. Roesky, D. Kratzert, K. Meindl, J. Henn, D. Stalke, J.-P. Demers, A. Lange, Angew. Chem. Int. Ed. 2011, 50, 2322-2325.

[14] "Reaction of a base-stabilized bis(silylene) $\left[\mathrm{PhC}\left(\mathrm{N}^{t} \mathrm{Bu}\right)_{2} \mathrm{Si}_{2}\right.$ with cyclooctatetraene without cleavage of the Si-Si bond", S. S. Sen, S. Khan, D. Kratzert, H. W. Roesky, D. Stalke, Eur. J. Inorg. Chem. 2011, 1370-1373.

[13] "Synthesis and Structure of $\left[\left\{\mathrm{PhC}\left(\mathrm{N}^{t} \mathrm{Bu}\right)_{2}\right\}_{2} \mathrm{Ge}_{2}(\mu-\mathrm{S})_{2} \mathrm{Cl}_{2}\right]$ and a Germanium Dithiocarboxylate Analogue", S. S. Sen, R. S. Ghadwal, D. Kratzert, D. Stern, H. W. Roesky, D. Stalke, Organometallics 2011, 30, 1030-1033.

[12] "Phenalenyl-based ligand for transition metal chemistry: application in Henry reaction", A. Mukherjee, T. K. Sen, S. K. Mandal, D. Kratzert, D. Stalke, A. Döring, C. Schulzke, Journal of Chemical Sciences 2011, 123, 139-144.

[11] "Intermolecular Twofold Carbopalladation/Cyclization Sequence to Access Chromans and Isochromans from Carbohydrates", M. Leibeling, B. Milde, D. Kratzert, D. Stalke, D. B. Werz, Chem. Eur. J. 2011, 17, 9888-9892. 
[10] "Experimental charge density distribution of non-coordinating $s p^{3}$ carbanions in $\left[M g\left\{\left(p z^{*}\right)_{3} C\right\}_{2}\right]^{\prime \prime}$, D. Kratzert, D. Leusser, D. Stern, J. Meyer, F. Breher, D. Stalke, Chem. Commun. 2011, 47, 2931-2933.

[9] "Synthesis of stable silicon heterocycles by reaction of organic substrates with a chlorosilylene $\left[\mathrm{PhC}\left(\mathrm{N}^{t} \mathrm{Bu}\right)_{2} \mathrm{SiCl}\right]$ ", S. Khan, S. S. Sen, D. Kratzert, G. Tavčar, H. W. Roesky, D. Stalke, Chem. Eur. J. 2011, 17, 4283-4290.

[8] "A simple system with many structural variants: a reexamination of (amine)halogenidosilver(I) complexes", C. Woelper, M. D. P. Bastardes, I. Dix, D. Kratzert, P. G. Jones, Z. Naturforsch., B: Chem. Sci. 2010, 65, 647-673.

[7] "Reactions of a Bis-silylene ( $L S i-S i L, L=P h C\left(N^{t} B u\right)_{2}$ ) and a Heteroleptic Chloro Silylene (LSiCl) with Benzil: Formation of Bis(siladioxolene) and Monosiladioxolene Analogue with FiveCoordinate Silicon Atoms in Both Ring Systems", G. Tavčar, S. S. Sen, H. W. Roesky, J. Hey, D. Kratzert, D. Stalke, Organometallics 2010, 29, 3930-3935.

[6] "Synthesis of a Stable Four-Membered $\mathrm{Si}_{2} \mathrm{O}_{2}$ Ring and a Dimer with Two Four-Membered $\mathrm{Si}_{2} \mathrm{O}_{2}$ Rings Bridged by Two Oxygen Atoms, with Five-Coordinate Silicon Atoms in Both Ring Systems", S. S. Sen, G. Tavčar, H. W. Roesky, D. Kratzert, J. Hey, D. Stalke, Organometallics 2010, 29, 2343-2347.

[5] "Reactivity studies of a GeL-GeL compound with and without cleavage of the Ge-Ge bond", S. S. Sen, D. Kratzert, D. Stern, H. W. Roesky, D. Stalke, Inorg. Chem. 2010, 49, 5786-5788.

[4] "Hybrids of sugars and aromatics: A Pd-catalyzed modular approach to chromans and isochromans", M. Leibeling, D. C. Koester, M. Pawliczek, D. Kratzert, B. Dittrich, D. B. Werz, Bioorg. Med. Chem. 2010, 18, 3656-3667.

[3] "One Pot Synthesis of Disilatricycloheptene Analogue and Jutzi's Disilene", S. Khan, S. S. Sen, H. W. Roesky, D. Kratzert, R. Michel, D. Stalke, Inorg. Chem. 2010, 49, 9689-9693.

[2] "Convenient Access to Monosilicon Epoxides with Pentacoordinate Silicon", R. S. Ghadwal, S. S. Sen, H. W. Roesky, M. Granitzka, D. Kratzert, S. Merkel, D. Stalke, Angew. Chem. Int. Ed. 2010, 49, 3952-3955.

[1] "Efficient Formal Total Synthesis of the Erythrina Alkaloid (+)-Erysotramidine, Using a Domino Process", L. F. Tietze, N. Tölle, D. Kratzert, D. Stalke, Org. Lett. 2009, 11, 5230-5233. 


\section{Literature}

[1] W. Friedrich, P. Knipping, M. von Laue, in Sitzungsbericht der kaiserlich Akademischen Wissenschaften München, Sitzb. kais. Akad. Wiss., München, 1912, pp. 303-322.

[2] W. H. Bragg, W. L. Bragg, Proc. R. Soc. A 1913, 88, 428-438.

[3] M. v. Laue, in Nobel Lectures, Physics, Vol. 1901-1921, 1914.

[4] E. Keller, Chem. Unserer Zeit, 1982, 16, 116-123.

[5] E. Keller, Chem. Unserer Zeit, 1982, 16, 71-88.

[6] P. Müller, R. Herbst-Irmer, A. L. Spek, T. R. Schneider, M. R. Sawaya in IUCr Texts on Crystallography, (Eds.: P. Müller), Oxford University Press, Oxford (England), 2006.

[7] R. McWeeny, Acta Crystallogr. 1953, 6, 631-637.

[8] S. Parsons, P. Pattison, H. D. Flack, Acta Crystallogr. 2012, A68, 736-749, and references therein.

[9] M. Baake, U. Grimm, D. H. Warrington, J. Phys. Chem. A 1994, 27, 2669-2674.

[10] T. S. Koritsanszky, P. Coppens, Chem. Rev. 2001, 101, 1583-1627.

[11] J. D. Dunitz, V. Schomaker, K. N. Trueblood, J. Phys. Chem. 1988, 92, 856-867.

[12] D. Watkin, J. Appl. Crystallogr. 2008, 41, 491-522.

[13] F. H. Allen, I. J. Bruno, Acta Crystallogr. 2010, B66, 380-386.

[14] R. F. Stewart, J. Chem. Phys. 1972, 57, 1664-1668.

[15] R. F. Stewart, J. Chem. Phys. 1973, 58, 1668-1676.

[16] N. K. Hansen, P. Coppens, Acta Crystallogr. 1978, A34, 909-921.

[17] A. Volkov, P. Coppens, Acta Crystallogr. 2001, A57, 395-405.

[18] C. P. Brock, J. D. Dunitz, F. L. Hirshfeld, Acta Crystallogr. 1991, B47, 789-797.

[19] B. Dittrich, T. Koritsanszky, P. Luger, Angew. Chem. Int. Ed. 2004, 43, 2718-2721; Angew. Chem. 2004, 116.

[20] B. Dittrich, C. B. Hübschle, P. Luger, M. A. Spackman, Acta Crystallogr. 2006, D62, 1325-1335.

[21] C. B. Hübschle, P. Luger, B. Dittrich, J. Appl. Crystallogr. 2007, 40, 623-627.

[22] B. Dittrich, C. B. Hübschle, K. Pröpper, F. Dietrich, T. Stolper, J. J. Holstein, Acta Crystallogr. 2013, B69, 91-104.

[23] A. Volkov, X. Li, T. Koritsanszky, P. Coppens, J. Phys. Chem. 2004, A108, 4283-4300.

[24] P. M. Dominiak, A. Volkov, X. Li, M. Messerschmidt, P. Coppens, J. Chem. Theory Comput. 2007, 3, 232247.

[25] A. Volkov, M. Messerschmidt, P. Coppens, Acta Crystallogr. 2007, D63, 160-170.

[26] K. N. Jarzembska, P. M. Dominiak, Acta crystallographica. Section A, Foundations of crystallography 2012, 68, 139-147.

[27] C. Jelsch, V. Pichon-Pesme, C. Lecomte, A. Aubry, Acta Crystallogr. 1998, D54, 1306-1318.

[28] S. Domagala, B. Fournier, D. Liebschner, B. Guillot, C. Jelsch, Acta crystallographica. Section A, Foundations of crystallography 2012, 68, 337-351.

[29] V. R. Hathwar, T. S. Thakur, T. N. G. Row, G. R. Desiraju, Cryst. Growth Des. 2011, 11, 616-623.

[30] E. J. Corey, Pure Appl. Chem. 1967, 14, 30-37.

[31] M. Roux, R. Daudel, Compt. Rend. Acad. Sci. 1955, 240, 90-92.

[32] P. Coppens, Science 1967, 158, 1577-1579.

[33] R. F. W. Bader, T.-H. Tang, Y. Tal, F. W. Biegler-König, J. Am. Chem. Soc. 1982, 104, 946-952.

[34] R. F. W. Bader, H. Essén, J. Chem. Phys. 1984, 80, 1943-1960.

[35] R. F. W. Bader, P. J. MacDougall, J. Am. Chem. Soc. 1985, 107, 6788-6795.

[36] R. F. W. Bader, Atoms in Molecules - A Quantum Theory, Oxford University Press, New York, 1990.

[37] C. F. Matta, R. F. W. Bader, J. Phys. Chem. 2006, A110, 6365-6371.

[38] R. F. W. Bader, J. Phys. Chem. 2009, A113, 10391-10396.

[39] R. F. W. Bader, J. Phys. Chem. 1998, A102, 7314-7323.

[40] D. Cremer, E. Kraka, T. S. Slee, R. F. W. Bader, C. D. H. Lau, T. T. Nguyen-Dang, P. J. MacDougall, J. Am. Chem. Soc. 1983, 105, 5069-5075.

[41] P. Coppens, X-Ray Charge Densities and Chemical Bonding, Oxford University Press, Oxford, 1997.

[42] D. Stalke, Chem. Eur. J. 2011, 17, 9264-9278.

[43] U. Flierler, D. Stalke, L. J. Farrugia, in Modern Charge-Density Analysis (Eds.: C. Gatti, P. Macchi), Springer, Heidelberg, London, New York, 2012, pp. 435-467. 
[44] D. Stalke, in Electron density and chemical bonding I (Experimental charge density studies) and II (Theoretical charge density studies) in Struct. Bonding, Vol. 146 (Eds.: D. Stalke), Springer, Berlin, New York, 2012.

[45] T.-H. Tang, R. F. W. Bader, P. J. MacDougall, Inorg. Chem. 1985, 24, 2047-2053.

[46] R. F. W. Bader, T. S. Slee, D. Cremer, E. Kraka, J. Am. Chem. Soc. 1983, 105, 5061-5068.

[47] J. R. Cheeseman, M. T. Caroll, R. F. W. Bader, Chem. Phys. Lett. 1988, 143, 450-458.

[48] K. Meindl, J. Henn, Acta Crystallogr. 2008, A64, 404-418.

[49] I. N. Bronstein, K. A. Semendjajew, G. Musiol, H. Mühlig, in Taschenbuch der Mathematik, Verlag Harry Deutsch, Frankfurt am Main, 1993.

[50] T. Kottke, D. Stalke, J. Appl. Crystallogr. 1993, 26, 615-619.

[51] D. Stalke, Chem. Soc. Rev. 1998, 27, 171-178.

[52] T. Schulz, K. Meindl, D. Leusser, D. Stern, J. Graf, C. Michaelsen, M. Ruf, G. M. Sheldrick, D. Stalke, J. Appl. Crystallogr. 2009, 42, 885-891.

[53] W. Cochran, Acta Crystallogr. 1969, A25, 95-101.

[54] K. D. Rouse, M. J. Cooper, Acta Crystallogr. 1969, A25, 615-621.

[55] S. Chandra, M. P. Hemkar, Acta Crystallogr. 1973, A29, 25-28.

[56] E. D. Stevens, Acta Crystallogr. 1974, A30, 184-189.

[57] A. W. Stevenson, J. Harada, Acta Crystallogr. 1983, A39, 202-207.

[58] V. Zavodnik, A. Stash, V. Tsirelson, R. D. Vries, D. Feil, Acta Crystallogr. 1999, B55, 45-54.

[59] T. Lippmann, J. R. Schneider, J. Appl. Crystallogr. 2000, 33, 156-167.

[60] B. B. He, in Two-dimensional X-Ray Diffraction, wiley VCH, Hoboken, New Jersey, 2011.

[61] P. Müller, Crystallography Reviews 2009, 15, 57-83.

[62] SAINT, v7.68A, Bruker-AXS, Madison (WI), 2009.

[63] W. Kabsch, J. Appl. Crystallogr. 1988, 21, 916-924.

[64] J. Zaleski, G. Wu, P. Coppens, J. Appl. Crystallogr. 1998, 31, 302-304.

[65] G. Wu, B. L. Rodrigues, P. Coppens, J. Appl. Crystallogr. 2002, 35, 356-359.

[66] L. V. Azaroff, Acta Crystallogr. 1955, 8, 701-704.

[67] K. Diederichs, Acta Crystallogr. 2010, D66, 733-740.

[68] A. Stash, DRKplot for XD and SHELX, Moscow, 2007.

[69] G. M. Sheldrick, unfinished program, Göttingen, 2013.

[70] G. M. Sheldrick, Acta Crystallogr. 2008, A64, 112-122.

[71] G. M. Sheldrick, SADABS, v2008/2, Göttingen, 2008.

[72] F. H. Allen, Acta Crystallogr. 1986, B42, 515-522.

[73] V. L. Deringer, V. Hoepfner, R. Dronskowski, Cryst. Growth Des. 2012, 12, 1014-1021.

[74] gnuplot, 2012, http://www.gnuplot.info/.

[75] P. Jutzi, Chem. Unserer Zeit, 1981, 15, 149-154.

[76] A. G. Brook, F. Abdesaken, B. Gutekunst, G. Gutekunst, R. K. Kallury, J. Chem. Soc., Chem. Commun. 1981, 0, 191-192.

[77] R. West, M. J. Fink, J. Michl, Science 1981, 214, 1343-1344.

[78] R. West, Angew. Chem. Int. Ed. 1987, 26, 1201-1210; Angew. Chem. 1987, 99, 1231-1241.

[79] W. Kutzelnigg, Angew. Chem. Int. Ed. Engl. 1984, 25, 272-295; Angew. Chem. 1984, 96, 262-286.

[80] G. Raabe, J. Michl, Chem. Rev. 1985, 85, 419-509.

[81] H. G. M. Driess, Angew. Chem. Int. Ed. Engl. 1996, 35, 828-856; Angew. Chem. 1996, 108, 900-9029.

[82] P. P. Power, Chem. Rev. 1999, 99, 3463-3503.

[83] N. O. J. Malcolm, R. J. Gillespie, P. L. A. Popelier, J. Chem. Soc., Dalton Trans. 2002, 3333-3341.

[84] R. C. Fischer, P. P. Power, Chem. Rev. 2010, 110, 3877-3923.

[85] U. Öpik, M. H. L. Pryce, Proc. Roy. Soc. 1957, A238, 425-428.

[86] R. G. Pearson, J. Mol. Struct. (Theochem) 1983, 103, 25-34.

[87] A. Sekiguchi, R. Kinjo, M. Ichinohe, Science 2004, 305, 1755-1757.

[88] N. Wiberg, S. K. Vasisht, G. Fischer, P. Mayer, Z. Anorg. Allg. Chem. 2004, 630, 1823-1828.

[89] A. Sekiguchi, Pure Appl. Chem. 2008, 80, 447-457.

[90] M. Weidenbruch, S. Willms, W. Saak, G. Henkel, Angew. Chem. Int. Ed. Engl. 1997, 36, 2503-2504; Angew. Chem. 1997, 109, 2612-2613.

[91] K. Wakita, N. Tokitoh, R. Okazaki, S. Nagase, Angew. Chem. Int. Ed. 2000, 39, 634-636; Angew. Chem. 2000, 112, 648-650

[92] Y. Kabe, K. Ohkubo, H. Ishikawa, W. Ando, J. Am. Chem. Soc. 2000, 122, 3775-3776. 
[93] S. S. Sen, H. W. Roesky, K. Meindl, D. Stern, J. Henn, A. C. Stückl, D. Stalke, Chem. Commun. 2010, 46, 5873-5875.

[94] R. Kinjo, M. Ichinohe, A. Sekiguchi, N. Takagi, M. Sumimoto, S. Nagase, J. Am. Chem. Soc. 2007, 129, 7766-7767.

[95] N. Wiberg, H. Auer, H. Nöth, J. Knizek, K. Polborn, Angew. Chem. Int. Ed. 1998, 37, 2869-2872; Angew. Chem. 1998, 110, 3030-3033.

[96] K. Takanashi, V. Y. Lee, T. Matsuno, M. Ichinohe, A. Sekiguchi, J. Am. Chem. Soc. 2005, 127, 5768-5769.

[97] K. Takanashi, V. Y. Lee, M. Ichinohe, A. Sekiguchi, Angew. Chem. Int. Ed. 2006, 45, 3269-3272; Angew. Chem. 2006, 118, 3347-3350.

[98] K. Takanashi, V. Y. Lee, M. Ichinohe, A. Sekiguchi, Eur. J. Inorg. Chem. 2007, 5471-5474.

[99] V. Y. Lee, K. Takanashi, T. Matsuno, M. Ichinohe, A. Sekiguchi, J. Am. Chem. Soc. 2004, 126 4758-4759.

[100] V. Y. Lee, K. Takanashi, R. Kato, T. Matsuno, M. Ichinohe, A. Sekiguchi, J. Organomet. Chem. 2007, 692, 2800-2810.

[101] Y. Apeloig, Science 2011, 331, 1277-1278.

[102] K. Suzuki, T. Matsuo, D. Hashizume, H. Fueno, K. Tanaka, K. Tamao, Science 2011, 331, 1306-1309.

[103] C. Gellini, P. R. Salvi, Symmetry 2010, 2, 1846-1924.

[104] G. Fischer, V. Huch, P. Mayer, S. K. Vasisht, M. Veith, N. Wiberg, Angew. Chem. Int. Ed. 2005, 44, 78847887; Angew. Chem. 2005, 117, 8096-8099.

[105] N. Wiberg, C. M. M. Finger, K. Polborn, Angew. Chem. Int. Ed. 1993, 32, 1054-1056; Angew. Chem. 1993, 105, 1140-1142.

[106] K. Abersfelder, A. J. P. White, H. S. Rzepa, D. Scheschkewitz, Science 2010, 327, 564-566.

[107] R. J. F. Berger, H. S. Rzepa, D. Scheschkewitz, Angew. Chem. Int. Ed. 2010, 49, 10006-10009; Angew. Chem. 2010, 122, 10203-10206.

[108] K. Abersfelder, A. J. P. White, R. J. F. Berger, H. S. Rzepa, D. Scheschkewitz, Angew. Chem. Int. Ed. 2011, 50, 7936-7939; Angew. Chem. 2011, 123, 8082-8086.

[109] A. Sekiguchi, V. Y. Lee, Chem. Rev. 2003, 103, 1429-1447.

[110] M. Kira, T. Iwamoto, Adv. Organomet. Chem. 2006, 54, 73-148.

[111] D. Scheschkewitz, Chem. Eur. J. 2009, 15, 2476-2485.

[112] Y. Wang, G. H. Robinson, Chem. Commun. 2009, 5201-5213

[113] V. Y. Lee, A. Sekiguchi, in Organometallic Compunds of Low-Coordinated Si, Ge, Sn and Pb, Wiley, Chichester, 2010.

[114] T. Iwamoto, K. Uchiyama, C. Kabuto, M. Kira, Chem. Lett. 2007, 36, 368-369.

[115] Q. Zhang, S. Yue, X. Lu, Z. Chen, R. Huang, L. Zheng, P. v. R. Schleyer, J. Am. Chem. Soc. 2009, 131, 97899799.

[116] E. Niecke, A. Fuchs, F. Baumeister, M. Nieger, W. W. Schoeller, Angew. Chem. Int. Ed. Engl. 1995, 34, 555-557; Angew. Chem. 1995, 107, 640-642.

[117] D. Nied, R. Köppe, W. Klopper, H. Schnöckel, F. Breher, J. Am. Chem. Soc. 2010, 132, 10264-10265.

[118] D. Nied, P. Oña-Burgos, W. Klopper, F. Breher, Organometallics 2011, 30, 1419-1428.

[119] D. Scheschkewitz, Angew. Chem. Int. Ed. 2005, 44, 2954-2956; Angew. Chem. 2005, 117, 3014-3016.

[120] D. Jose, A. Datta, J. Phys. Chem. 2012, C116, 24639-24648.

[121] S. Nagase, T. Kudo, M. Aoki, J. Chem. Soc., Chem. Commun. 1985, 1121-1122.

[122] Y. Apeloig, M. Karni in The Chemistry of Organosilicon Compounds (Eds.: S. Patai, Z. Rappoport), Wiley, New York, 1989, pp. $151-166$.

[123] G. Raabe, J. Michl in The Chemistry of Organosilicon Compounds (Eds.: S. Patai, Z. Rappoport), Wiley, New York, 1989, pp. 1102-1108.

[124] A. G. Brook, M. A. Brook, Adv. Organomet. Chem. 1996, 39, 71-158.

[125] P. v. R. Schleyer, H. Jiao, N. J. R. v. E. Hommes, V. G. Malkin, O. L. Malkina, J. Am. Chem. Soc. 1997, 119, 12669-12670.

[126] K. K. Baldridge, O. Uzan, J. M. L. Martin, Organometallics 2000, 19, 1477-1487.

[127] J. C. Santos, P. Fuentealba, Chem. Phys. Lett. 2007, 443, 439-442.

[128] A. F. Sax, J. Kalcher, R. Janoschek, J. Comp. Chem. 1988, 9, 564-577.

[129] S. Slanina, Chem. Phys. Lett. 1989, 161, 175-178.

[130] R. Janoschek, J. Inorg. Organomet. P. 1995, 5, 155-161.

[131] A. Grassi, G. M. Lombardo, R. Pucci, G. G. N. Angilella, F. Bartha, N. H. March, Chem. Phys. 2004, 297, 13-19.

[132] C. Gerdes, T. Müller, Angew. Chem. Int. Ed. 2010, 49, 4860-4862; Angew. Chem. 2010, 122, 4978-4981.

[133] P. v. R. Schleyer, Chem. Rev. 2001, 101, 1115-1117. 
[134] P. v. R. Schleyer, Chem. Rev. 2005, 105, 3433-3435.

[135] E. Matito, J. Poater, M. Sola, P. v. R. Schleyer in Aromaticity and Chemical reactivity, (Eds.: P. K. Chattaraj), Taylor and Francis/CRC Press, Boca Raton, 2009, pp. 419-438.

[136] K. Abersfelder, A. Russell, H. S. Rzepa, A. J. P. White, P. R. Haycock, D. Scheschkewitz, J. Am. Chem. Soc. 2012, 134, 16008-16016.

[137] A. Volkov, P. Macchi, L. J. Farrugia, C. Gatti, P. R. Mallinson, T. Richter, T. Koritsanszky, XD2006, 2006.

[138] K. Meindl, J. Henn, N. Kocher, D. Leusser, K. A. Zachariasse, G. M. Sheldrick, T. Koritsanszky, D. Stalke, J. Phys. Chem. 2009, A113, 9684-9691.

[139] C. B. Hübschle, B. Dittrich, J. Appl. Crystallogr. 2011, 44, 238-240.

[140] D. Jayatilaka, Chem. Phys. Lett. 1994, 230, 228-230.

[141] D. Jayatilaka, D. J. Grimwood, Computional Science - ICCS 2003, 4, 142-151.

[142] M. J. Frisch, et al., Gaussian 09, Revision C.01, Gaussian, Inc., Wallingford CT, 2010.

[143] Z. Su, P. Coppens, Acta Crystallogr. 1998, A54, 646-652.

[144] P. Macchi, P. Coppens, Acta Crystallogr. 2001, A57, 656-662.

[145] J. M. Bąk, P. M. Dominiaka, C. C. Wilson, K. Woźniak, Acta Crystallogr. 2009, A65, 490-500.

[146] A. Volkov, Y. A. Abramov, P. Coppens, Acta Crystallogr. 2001, A57, 272-282.

[147] N. Kocher, D. Leusser, A. Murso, D. Stalke, Chem. Eur. J. 2004, 10, 3622-3631.

[148] D. Leusser, J. Henn, N. Kocher, B. Engels, D. Stalke, J. Am. Chem. Soc. 2004, 126, 1781-1793.

[149] P. M. Dominiak, P. Coppens, Acta Crystallogr. 2006, A62, 224-227.

[150] C. A. Coulson, W. E. Moffitt, J. Chem. Phys. 1947, 15, 151-151.

[151] T. Koritsanszky, J. Buschmann, P. Luger, J. Phys. Chem. 1996, 100, 10547-10553.

[152] M. Messerschmidt, S. Scheins, L. Grubert, M. Pätzel, G. Szeimies, C. Paulmann, P. Luger, Angew. Chem. Int. Ed. 2005, 44, 3925-3928; Angew. Chem. 2005, 117, 3993-3997.

[153] R. B. C. Gustav. Albrecht J. Am. Chem. Soc. 1939, 61, 1087-1103.

[154] I. Rozas, I. Alkorta, J. Elguero, J. Phys. Chem. A 1998, 102, 9925-9932.

[155] D. Leusser, PhD thesis, Germany (Würzburg), 2002.

[156] D. Leusser, B. Walfort, D. Stalke, Angew. Chem. 2002, 114, 2183-2186.

[157] T. Steiner, Angew. Chem. Int. Ed. 2002, 41, 48-78; Angew. Chem. 2002, 114, 50-80.

[158] S. Shaik, D. Danovich, B. Silvi, D. L. Lauvergnat, P. C. Hiberty, Chem. Eur. J. 2005, 11, 6358-6371.

[159] W. Wu, J. Gu, J. Song, S. Shaik, P. C. Hiberty, Angew. Chem. Int. Ed. 2009, 48, 1407-1410; Angew. Chem. 2009, 121, 1435-1438.

[160] F. Breher, Coord. Chem. Rev. 2007, 251, 1007-1043.

[161] R. F. W. Bader, T. T. Nguyen-Dang, Y. Tal, Rep. Prog. Phys. 1981, 44, 893-948.

[162] W. Scherer, P. Sirsch, D. Shorokhov, G. S. McGrady, S. A. Mason, M. G. Gardiner, Chem. Eur. J. 2002, 8, 2324-2334.

[163] N. Kocher, C. Selinka, D. Leusser, D. Kost, I. Kalikhman, D. Stalke, Z. Anorg. Allg. Chem. 2004, 630, $1777-$ 1793.

[164] M. Veith, A. Spaniol, J. PöhImann, F. Gross, V. Huch, Chem. Ber. 1993, 12, 2625-2635.

[165] M. A. Beswick, D. S. Wright, Coord. Chem. Rev. 1998, 176, 373-406.

[166] R. Fleischer, S. Freitag, F. Pauer, D. Stalke, Angew. Chem. Int. Ed. Engl. 1996, 35, 204-207; Angew. Chem. 1996, 108, 208-211

[167] R. Fleischer, D. Stalke, Coord. Chem. Rev. 1998, 176, 431-450.

[168] J. K. Brask, T. Chivers, Angew. Chem. Int. Ed. 2001, 40, 3960-3976; Angew. Chem. 2001, 113, 4082-4098.

[169] D. Stalke, Chem. Commun. 2012, 48, 9559-9573.

[170] L. H. Gade, Chem. Commun. 2000, 173-181.

[171] L. H. Gade, Acc. Chem. Res. 2002, 35, 575-582.

[172] L. H. Gade, S. Bellemin-Laponnaz, Chem. Eur. J. 2008, 14, 4142-4152.

[173] W. Hückel, H. Bretschneider, Chem. Ber. 1937, 70, 2024-2026.

[174] S. Trofimenko, J. Am. Chem. Soc. 1966, 88 1842-1844.

[175] S. Trofimenko, J. Am. Chem. Soc. 1970, 92 5118-5126.

[176] S. Trofimenko, Chem. Rev. 1993, 93, 943-980.

[177] E. R. Humphrey, K. L. V. Mann, Z. R. Reeves, A. Behrendt, J. C. Jeffery, J. P. Maher, J. A. McCleverty, M. D. Ward, New J. Chem. 1999, 23, 417-423.

[178] S. Trofimenko, J. Chem. Educ. 2005, 82, 1715-1720.

[179] J. Klingele, S. Dechert, F. Meyer, Coord. Chem. Rev. 2009, 253, 2698-2741.

[180] A. Steiner, D. Stalke, J. Chem. Soc., Chem. Commun. 1993, 1702-1704. 
[181] A. Steiner, D. Stalke, Angew. Chem. Int. Ed. Engl. 1995, 34, 1752-1755; Angew. Chem. 1995, 107, 19081910.

[182] F. Reichart, M. Kischel, K. Zeckert, Chemistry 2009, 15, 10018-10020.

[183] F. Armbruster, I. Fernández, F. Breher, Dalton transactions 2009, 5612-5626.

[184] P. K. Byers, N. Carr, F. G. A. Stone, J. Chem. Soc., Dalton Trans. 1990, 3701-3708.

[185] P. K. Byers, F. G. A. Stone, J. Chem. Soc., Dalton Trans. 1991, 93-99.

[186] D. Hoffmann, W. Bauer, P. v. R. Schleyer, U. Pieper, D. Stalke, Organometallics 1993, 12, 1193-1200.

[187] U. Pieper, D. Stalke, Organometallics 1993, 12, 1201-1206.

[188] H. Gornitzka, D. Stalke, Organometallics 1994, 13, 4398-4405.

[189] H. Gornitzka, D. Stalke, Angew. Chem. Int. Ed. Engl. 1994, 33, 693-695; Angew. Chem. 1994, 106, 695698.

[190] D. L. Reger, Comm. Inorg. Chem. 1997, 21, 1-28.

[191] W. Kläui, M. Berghahn, G. Rheinwald, H. Lang, Angew. Chem. Int. Ed. 2000, 39, 2464-2466 Angew. Chem. 2000, 112, 2590-2592.

[192] W. Kläui, D. Schramm, W. Peters, G. Rheinwald, H. Lang, Eur. J. Inorg. Chem. 2001, 2001, 1415-1424.

[193] S. C. Lawrence, M. E. G. Skinner, J. C. Green, P. Mountford, Chem. Commun. 2001, 705-706.

[194] F. Breher, J. Grunenberg, S. C. Lawrence, P. Mountford, H. Rüegger, Angew. Chem. Int. Ed. 2004, 43, 2521-2524; Angew. Chem. 2004, 116, 2575-2578.

[195] L. Benisvy, R. Wanke, M. L. Kuznetsov, M. F. C. Guedes da Silva, A. J. L. Pombeiro, Tetrahedron 2009, 65, 9218-9223.

[196] H. R. Bigmore, S. R. Dubberley, M. Kranenburg, S. C. Lawrence, A. J. Sealey, J. D. Selby, M. A. Zuideveld, A. R. Cowley, P. Mountford, Chem. Commun. 2006, 436-438

[197] F. Baier, Z. Fei, H. Gornitzka, A. Murso, S. Neufeld, M. Pfeiffer, I. Rüdenauer, A. Steiner, T. Stey, D. Stalke, J. Organomet. Chem. 2002, 661, 111-127.

[198] I. Kuzu, I. Krummenacher, J. Meyer, F. Armbruster, F. Breher, Dalton transactions 2008, 5836-5865.

[199] I. Krummenacher, H. Rüegger, F. Breher, Dalton transactions 2006, 1073-1081.

[200] H. R. Bigmore, J. Meyer, I. Krummenacher, H. Rüegger, E. Clot, P. Mountford, F. Breher, Chemistry 2008, 14, 5918-5934.

[201] I. Kuzu, I. Krummenacher, I. J. Hewitt, Y. Lan, V. Mereacre, A. K. Powell, P. Höfer, J. Harmer, F. Breher, Chemistry 2009, 15, 4350-4365.

[202] M. G. Cushion, J. Meyer, A. Heath, A. D. Schwarz, I. Fernández, F. Breher, P. Mountford, Organometallics 2010, 29, 1174-1190.

[203] Bruker APEX v2011.4-1, WI, USA, Madison, 2011.

[204] R. H. Blessing, Acta Crystallogr. 1995, A51, 33-38.

[205] E. Clementi, D. L. Raimondi, J. Chem. Phys. 1963, 38, 2686-2689.

[206] R. F. Stewart, J. Chem. Phys. 1969, 51, 4569-4577.

[207] E. Clementi, C. Roetti, Atom. Data Nuc. Data Tab. 1974, 14, 177-478.

[208] F. L. Hirshfeld, Acta Crystallogr. 1976, A32, 239-244.

[209] H. Ott, U. Pieper, D. Leusser, U. Flierler, J. Henn, D. Stalke, Angew. Chem. Int. Ed. 2009, 48, 2978-2982; Angew. Chem. 2009, 121, 3022-3026.

[210] H. R. Bigmore, J. Meyer, I. Krummenacher, H. Rüegger, E. Clot, P. Mountford, F. Breher, Chem. Eur. J. 2008, 14, 5918 - 5934.

[211] U. Flierler, D. Leusser, H. Ott, G. Kehr, G. Erker, S. Grimme, D. Stalke, Chemistry 2009, 15, 4595-4601.

[212] S. Deuerlein, D. Leusser, U. Flierler, H. Ott, D. Stalke, Organometallics 2008, 27, 2306-2315.

[213] D. Kratzert, Diploma Thesis, Deutschland (Göttingen), 2008.

[214] E. Bergin, Annual Reports Section "B" (Organic Chemistry) 2012, 108, 353.

[215] C. Moberg, Israel Journal of Chemistry 2012, 52, 653-662.

[216] I. Chorkendorff, J. W. Niemantsverdriet, in Concepts of Modern Catalysis and Kinetics, Wiley-VCH, Weinheim, 2007.

[217] J. K. Whitesell, Chem. Rev. 1989, 89, 1581-1590.

[218] M. J. Burk, R. L. Harlow, Angew. Chem. Int. Ed. Engl. 1990, 29, 1462-1464; Angew. Chem. 1990, 102, 1511-1513.

[219] C. Moberg, Angew. Chem. Int. Ed. 2006, 45, 4721-4723; Angew. Chem. 2006, 118, 4838-4840.

[220] W. H. Pirkle, T. C. Pochapsky, Chem. Rev. 1989, 89, 347-362.

[221] C. Moberg, Angew. Chem. Int. Ed. 1998, 37, 248-268; Angew. Chem. 1998, 110, $260-281$.

[222] L. Sacconi, F. Mani, Transition Met. Chem. (London) 1982, 8, 179-252.

[223] W. H. Hohman, D. J. Kountz, D. W. Meek, Inorg. Chem. 1986, 25, 616-623. 
[224] L. F. Rhodes, C. Sorato, L. M. Venanzi, F. Bachechi, Inorg. Chem. 1988, 27 604-610.

[225] C. Bianchini, A. Meli, M. Peruzzini, F. Vizza, F. Zanobini, Coord. Chem. Rev. 1992, 120, 193-208.

[226] K. W. Hellmanns, S. Friedricha, L. H. Gade, W.-S. Lib, M. McPartlin, Chem. Ber. 1995, 128, 29-34.

[227] B. Findeis, M. Schubart, L. H. Gade, F. Möller, I. Scowen, M. McPartlin, J. Chem. Soc., Dalton Trans. 1996, 125-132.

[228] K. W. Hellmann, L. H. Gade, A. Steiner, D. Stalke, F. Möller, Angew. Chem. Int. Ed. Engl. 1997, 36, 160163; Angew. Chem. 1997, 109, 99-102.

[229] L. H. Gade, P. Renner, H. Memmler, F. Fecher, C. H. Galka, M. Laubender, S. Radojevic, M. McPartlin, J. W. Lauher, Chem. Eur. J. 2001, 7, 2563-2580.

[230] M. Lutz, B. Findeis, M. Haukka, T. A. Pakkanen, L. H. Gade, Eur. J. Inorg. Chem. 2001, 3155-3162.

[231] H. Memmler, U. Kauper, L. H. Gade, D. Stalke, Organometallics 1996, 15, 3637-3639.

[232] J. G. Verkade, Acc. Chem. Res. 1993, 26, 483-489.

[233] R. R. Schrock, C. C. Cummins, T. Wilhelm, S. Lin, S. M. Reid, M. Kol, W. M. Davis, Organometallics 1996, 15, 1470-1476.

[234] R. R. Schrock, Acc. Chem. Res. 1997, 30, 9-16.

[235] S. Urgaonkar, J.-H. Xu, J. G. Verkade, J. Org. Chem. 2003, 68, 8416-8423.

[236] W. A. Nugent, R. L. Harlow, J. Am. Chem. Soc. 1994, 116, 6142-6148.

[237] H. Lütjens, G. Wahl, F. Möller, P. Knochel, J. Sundermeyer, Organometallics 1997, 16, 5869-5878.

[238] G. Desimoni, G. Faita, K. A. Jørgensen, Chem. Rev. 2011, 111, PR284-PR437.

[239] S. Bellemin-Laponnaz, L. H. Gade, Angew. Chem. Int. Ed. 2002, 33, 599-619; Angew. Chem. 2002, 114, 3623-3625.

[240] C. Foltz, M. Enders, S. Bellemin-Laponnaz, H. Wadepohl, L. H. Gade, Chem. Eur. J. 2007, 13, 5994-6008.

[241] L. Beaufort, A. Demonceau, A. F. Noels, Tetrahedron 2005, 61, 9025-9030.

[242] L. Beaufort, L. Delaude, A. F. Noels, Tetrahedron 2007, 63, 7003-7008.

[243] L. Beaufort, F. Benvenuti, L. Delaude, A. F. Noels, J. Mol. Catal. A - Chemical 2008, 283, 77-82.

[244] A. Jana, G. Schwab, H. W. Roesky, D. Stalke, Inorg. Chem. 2008, 47, 8990-8994.

[245] D. Gonbeau, G. Pfister-Guillouzo, M.-R. Mazières, M. Sanchez, Can. J. Chem. 1985, 63, 3242-3248.

[246] G. Trinquier, M. T. Ashby, Inorg. Chem. 1994, 116, 1306-1313.

[247] E. Niecke, D. Gudat, Angew. Chem. Int. Ed. Engl. 1991, 30, 217-237; Angew. Chem. 1991, 103, $251-270$.

[248] D. Gudat, H. M. Schiffner, M. Nieger, D. Stalke, A. J. Blake, H. Grondey, E. Niecke, J. Am. Chem. Soc. 1992, 114, 8857-8862.

[249] K. Dehnicke, F. Weller, Coord. Chem. Rev. 1997, 158, 103-169.

[250] R. Fleischer, D. Stalke, Inorg. Chem. 1997, 36, 2413-2419.

[251] C. M. Ong, P. McKarns, D. W. Stephan, Organometallics 1999, 18, 4197-4204.

[252] S. Wingerter, H. Gornitzka, G. Bertrand, D. Stalke, Eur. J. Inorg. Chem. 1999, 173-178.

[253] W.-P. Leung, Z.-X. Wang, H.-W. Li, Q.-C. Yang, T. C. W. Mak, J. Am. Chem. Soc. 2001, 123, 8123-8124.

[254] S. Wingerter, M. Pfeiffer, A. Murso, C. Lustig, T. Stey, V. Chandrasekhar, D. Stalke, J. Am. Chem. Soc. 2001, 123, 1381-1388.

[255] S. Wingerter, M. Pfeiffer, T. Stey, M. Bolboacá, W. Kiefer, V. Chandrasekhar, D. Stalke, Organometallics 2001, 20, 2730-2735.

[256] P. Wei, K. T. K. Chan, D. W. Stephan, Dalton transactions 2003, 3804-3810.

[257] K. T. K. Chan, L. P. Spencer, J. D. Masuda, J. S. J. McCahill, P. Wei, D. W. Stephan, Organometallics 2004, 23, 381-390.

[258] A. Murso, D. Stalke, Eur. J. Inorg. Chem. 2004, 4272-4277.

[259] Y. G. Gololobov, L. F. Kasukhin, Tetrahedron 1992, 48, 1353-1406.

[260] A. W. Johnson, Ylides and Imines of Phosphorus, Wiley, New York, 1993.

[261] A. A. Pedro Molina, Alejandro García, M.Carmen Ramírez de Arellano, Tetrahedron Lett. 1997, 38, 7613-7616.

[262] M. Sauthier, J. Forniés-Cámer, L. Toupet, R. Réau, Organometallics 2000, 19, 553-562.

[263] H.-R. Wu, Y.-H. Liu, S.-M. Peng, S.-T. Liu, Eur. J. Inorg. Chem. 2003, 2003, 3152-3159.

[264] A. Arques, D. Auñon, P. Molina, Tetrahedron Lett. 2004, 45, 4337-4340.

[265] T. Baumgartner, R. Réau, Chem. Rev. 2006, 106, 4681-4727.

[266] V. Cadierno, J. Diez, J. Garcia-Alvarez, J. Gimeno, N. Nebra, J. Rubio-Garcia, Dalton Trans 2006, 55935604.

[267] A. Buchard, B. Komly, A. Auffrant, X. F. L. Goff, P. L. Floch, Organometallics 2008, 27, 4380-4385.

[268] Y. G. Gololobov, I. N. Zhmurova, L. F. Kasukhin, Tetrahedron 1981, 37, 437-472.

[269] C. W. Tornøe, C. Christensen, M. Meldal, J. Org. Chem. 2002, 67, 3057-3064. 
[270] M. Meldal, C. W. Tornøe, Chem. Rev. 2008, 108, 2952-3015.

[271] V. V. Rostovtsev, L. G. Green, V. V. Fokin, K. B. Sharpless, Angew. Chem. Int. Ed. 2002, 41, 2596-2599; Angew. Chem. 2002, 114, 2708-2711.

[272] H. C. Kolb, M. G. Finn, K. B. Sharpless, Angew. Chem. Int. Ed. 2001, 40, 2004-2021; Angew. Chem. 2001 113, 2056-2075.

[273] R. Huisgen, Angew. Chem. Int. Ed. Engl. 1963, 2, 565-598; Angew. Chem. 1963, 75, 604-637.

[274] P. Mathew, A. Neels, M. Albrecht, J. Am. Chem. Soc. 2008, 130, 13534-13535.

[275] A. I. Poulain, D. Canseco-Gonzalez, R. Hynes-Roche, H. Müller-Bunz, O. Schuster, H. Stoeckli-Evans, A. Neels, M. Albrecht, Organometallics 2011, 30, 1021-1029.

[276] K. J. Kilpin, E. L. Gavey, C. J. McAdam, C. B. Anderson, S. J. Lind, C. C. Keep, K. C. Gordon, J. D. Crowley, Inorg. Chem. 2011, 50, 6334-6346.

[277] M. Empting, Nachrichten aus der Chemie 2013, 61, 33-36.

[278] M. Touaibia, T. C. Shiao, A. Papadopoulos, J. Vaucher, Q. Wang, K. Benhamioud, R. Roy, Chem. Commun. 2007, 380-382.

[279] J. Zana, H. Yana, Z.-f. Guoa, Z.-L. Lu, Inorg. Chem. Commun. 2010, 13, 1054-1056.

[280] L. H. Boudreau, N. Picot, J. Doiron, B. Villebonnet, M. E. Surette, G. A. Robichaud, M. Touaibia, New J. Chem. 2009, 33, 1932-1940.

[281] T. R. Chan, R. Hilgraf, K. B. Sharpless, V. V. Fokin, Org. Lett. 2004, 6, 2853-2855.

[282] H. W. Wanzlick, Angew. Chem. Int. Ed. Engl. 1962, 1, 75-80; Angew. Chem. 1962, 74, 129-134.

[283] K. Öfele, J. Organomet. Chem. 1968, 12, P42-P43.

[284] A. J. Arduengo, R. L. Harlow, M. Kline, J. Am. Chem. Soc. 1991, 113, 361-363.

[285] A. J. Arduengo, H. V. R. Dias, R. L. Harlow, M. Kline, J. Am. Chem. Soc. 1992, 114, 5530-5534.

[286] A. J. Arduengo, M. Tamm, J. C. Calabrese, F. Davidson, W. J. Marshall, Chem. Lett. 1999, 1021.

[287] A. J. Arduengo lii, R. Krafczyk, R. Schmutzler, H. A. Craig, J. R. Goerlich, W. J. Marshall, M. Unverzagt, Tetrahedron 1999, 55, 14523-14534.

[288] M. Tafipolsky, W. Scherer, K. Öfele, G. Artus, B. Pedersen, W. A. Herrmann, G. S. McGrady, J. Am. Chem. Soc. 2002, 124, 5865-5880.

[289] D. Bourissou, O. Guerret, F. P. Gabbai, G. Bertrand, Chem. Rev. 2000, 100, 39-92.

[290] P. Schwab, M. B. France, J. W. Ziller, R. H. Grubbs, Angew. Chem. Int. Ed. Engl. 1995, 34, 2039-2041; Angew. Chem. 1995, 107, 2179-2181.

[291] P. Schwab, R. H. Grubbs, J. W. Ziller, J. Am. Chem. Soc. 1996, 118, 100-110.

[292] M. S. Sanford, J. A. Love, R. H. Grubbs, J. Am. Chem. Soc. 2001, 123, 6543-6554.

[293] M. S. Sanford, M. Ulman, R. H. Grubbs, J. Am. Chem. Soc. 2001, 123, 749-750.

[294] T. M. Trnka, R. H. Grubbs, Acc. Chem. Res. 2001, 34, 18-29.

[295] R. H. Grubbs, Angew. Chem. Int. Ed. 2006, 45, 3760-3765; Angew. Chem. 2006, 123, 6870-6897.

[296] G. C. Vougioukalakis, R. H. Grubbs, Chem. Rev. 2009, 110, 1746-1787.

[297] W. A. Herrmann, Angew. Chem. Int. Ed. 2002, 41, 1290-1309; Angew. Chem. 2002, 114, 1342-1363.

[298] F. E. Hahn, Angew. Chem. Int. Ed. 2006, 45, 1348-1352; Angew. Chem. 2006, 118, 1374-1378.

[299] F. E. Hahn, M. C. Jahnke, Angew. Chem. Int. Ed. 2008, 47, 3122-3172; Angew. Chem. 2008, 120, 31663216.

[300] W. Schlenk in Die Methoden der Organischen Chemie, 2. Aufl. (Eds.: J. Houben), G. Thieme, Leipzig, 1924, pp. 720.

[301] T. T. Tidwell, Angew. Chem. Int. Ed. 2001, 40, 331-337; Angew. Chem. 2001, 113, 343-349.

[302] S. K. Brayshaw, J. W. Knight, P. R. Raithby, T. L. Savarese, S. Schiffers, S. J. Teat, J. E. Warren, M. R. Warren, J. Appl. Crystallogr. 2010, 43, 337-340. 


\section{Index}

$(3,+1) 5$

$(3,+3) 5$

$(3,-1) 5$

$(3,-3) 5$

[1.1.1]propellane 50, 51

$\left[\mathrm{Mg}\left\{\left(\mathrm{pz}^{*}\right)_{3} \mathrm{C}\right\}_{2}\right] 63$

1,2,3-triazoles 77

1,2-disilabenzene 25

1,3-dipolar cycloaddition 77

1,4-disila-(Dewar)-benzene 24

1,4-disilabenzene 24

AAC 77

absolute structure 1

absolute-configuration 1

anomalous signal 1

APEXII 64

aspherical atom form factors 3

aspherical description of the electron density 3

aspherical electron density 3

atom form factor 1

atomic basin 7

atomic densities 1

azide 77

beam stop 134, 135

biradical character 51

bond path 4

bond vector 6

$\mathrm{C}_{3}$-symmetrical ligands 62

cage critical point 52

charge shift bond 50

chemical concepts 4

chemical constraints 35

chemical symmetry 3

click reaction 77

closed shell 6

closed shell interactions 6

constrain 3

core density 2

correlations of parameters 65

COSMO 13

covalent 6

critical point 4

Cross Hair Alignment 132

CUAAC 77

data reduction 64

databases 3 deconvolution of motion 2

deformation density 57

diamagnetic current 30

Diederichs plot 12

difference electron density 64

diradical 28

disorder

dynamic disorder 48, 59

rotational disorder 64

disordered 31

DMSDA test 65,66

double bond rule 22

electron density 2

electrostatic potential 58

ellipticity 6, 69, 71

ELMAM 3

expansion-contraction parameters 65

flat and featureless 67

fractal dimension 8,67

fragments 3

glass fiber 10

Gradient field 41

gradient vector field 7, 52

harmonic approximation 2

Helijet 63

helium-cooled 134

Hessian matrix 5

hexasilabenzene 27, 29

hexasilaprismane 26

hydrogen atoms 65

hydrogen placement 16

IAM 3

iminophosphoranes 76

independent atom model 1, 2

inert atmosphere 10

invariom database 16, 32, 33

invariom model 32

invariom transfer 16

Invariome.in 33

Invariome.out 33

ionic 6

I $\mu$ S 10

Jahn-Teller distortion 26

jnk2RDA 60, 67

Laplacian 5

least-squares refinement 65 
local symmetry 3

magnetically induced probability density current 30

master file 33

MiTeGen 10

MolecoolQT 32

monopole 17

multipole model 3

multipole refinement $31,33,65$

NHC 77

$\mathrm{N}$-heterocyclic carbene 77

$\mathrm{N}$-heterocylic carbene 78

$\operatorname{NICS}(0) 30$

nitrogen heterocycles 69

omega-scan 64

oxidation state 53

paramagnetic current 30

parameters 3

Plotxd 18

$\mathrm{P}-\mathrm{N}$ bond 76

pre-defined multipole parameters 3

promolecule 16

pseudo Jahn-Teller 29

pyramidal carbanion 62

pyrazolyl 66

pyrazolyl ligands 62

QTAIM 4

Quantum theory of Atoms in Molecules 4

radial exponential distribution 1

rank 5

Refcheck 19

residual density 48

restraints $34,48,59$

riding model 34,65

ring-current 30

SADABS 14, 15, 64

SAINT 64

SBFA 3

scale factor $13,14,17$

scattering power 1

Schlenk-line 10

shared interaction 6

shared interactions 6

\section{SHELXT 15}

Si=Si double bond 23

$\mathrm{Si}_{6} \mathrm{H}_{6} 29$

Silabenzene 25

silapropellane 50

silicon 22, 42

Si-Si single bond 26

Slater functions 3

smart 600010

solvent molecule 48

spherical harmonics 2

starting model 3, 15, 33

static deformation density 4

static electron density 1

Staudinger reaction 77

structure factor 1

supersilyl 27

thermal-displacement distribution 2

TONTO 33

topological charge 7

topological parameters 70

transfer of density 3

Tripodal ligands 74

tris(pyrazolyl)methane 62

Trofimenco 62

TXS 10

valence density 2

valence shell charge concentrations 6

vibronic coupling 29

VSCC 42

weighting scheme 15

xd_part.aux 32

XD2006 17, 33, 65

XDLSM 65

X-ray diffraction 1

$\mathrm{X}$-ray radiation 1

X-Temp2 10

zero-flux surface 7

zeta values 48

к 2, 21, 65

א' 2, 21, 65

$\pi$-bond 6

$\pi$-character 6 
